\title{
HEAT AND MASS TRANSFER IN UNSATURATED SOILS DURING FREEZING
}

\author{
A Thesis \\ Submitted to the Faculty of Graduate Studies and Research \\ in Partial Fulfillment of the Requirements \\ For the Degree of \\ Master of Science \\ in the \\ Department of Civil Engineering \\ University of Saskatchewan \\ Saskatoon, Canada \\ by
}

Greg P. Newman

Fall, 1995

(C) Copyright Greg P. Newman, 1995. All Rights Reserved 
The author has agreed that the Library at the University of Saskatchewan may make this thesis freely available for inspection. Moreover, the author has agreed that permission for the extensive copying of the work recorded herein, in whole or in part, may be granted by the supervisors of this research or, in their absence, by the Head of the Department of Civil Engineering or the Dean of the College of Engineering. It is understood that due recognition will be given to the author of this thesis and to the University of Saskatchewan for any use of the material contained herein. Copying or publication or any other use of this thesis for financial gain without the approval of the University of Saskatchewan and the author's written approval is prohibited.

Requests for permission to copy, or to make any other use of material in this thesis, in whole or in part, should be addressed to:

\author{
Head of the Department of Civil Engineering \\ University of Saskatchewan \\ 57 Campus Drive \\ Saskatoon, Saskatchewan \\ Canada
}

S7N 5A9 


\section{ABSTRACT}

Experimental and field data have shown that large amounts of water can be redistributed from warmer soils to and behind an advancing freezing front. The mechanisms by which this occurs are becoming more understood, but the most appropriate method for analysing these mechanisms is not yet known. Various researchers have developed soil freezing models, but they are all limited to some extent and are not practical tools from a design or predictive modelling perspective. The objective of this research program is to develop unsaturated soil freezing theory from a geotechnical engineering perspective, and to verify the theory by modifying an existing non-freezing soil heat and mass transfer model.

In this study the SoilCover (MEND, 1993) model is modified to verify the theory and numerical solution. SoilCover (MEND, 1993) is a one-dimensional soil heat flow and mass transfer computer model used for designing protective covers over waste rock and tailings. These covers, if they remain saturated, significantly reduce oxygen infiltration into the waste material where it can combine with water to produce acid mine drainage. SoilCover (MEND, 1993) is not capable of modelling through the winter months when upper regions of the covers become subjected to freezing temperatures.

Unique to the modified soil freezing model is the method by which the coupled heat and mass equations are combined and solved. The numerical model uses a single, unique expression which describes the heat flow, mass transfer, and phase change phenomenon in the frozen or partially frozen soil zones. To derive the modified equation, the dependent suction variable in the mass transfer equation is re-written as a function of freezing point depression temperature using a Clapeyron type relationship that is obtained by combining soil freezing curve data with soil water characteristic curve data. The mass transfer 
equation is then re-written as a function of change in ice content and substituted into the ice content term of the heat transfer equation. The result is a single combined heat and mass transfer equation with one unknown variable, i.e., temperature. Once new temperatures are solved for over the current time step, suctions and ice contents are computed using back-substitution.

The revised model was verified using laboratory freezing test data collected at the University of Saskatchewan in 1977. During laboratory data modelling of three freezing tests, the average percent difference between measured and computed frost front positions was approximately $6 \%$. The average difference between measured and computed ice contents was approximately $7 \%$, and the average difference between measured and computed liquid water contents was approximately $14 \%$. These discrepancies were primarily due to errors in the estimated and measured soil thermal and hydraulic property functions.

Results of the laboratory data simulations suggest that the permeability versus suction relationship for an unsaturated soil also applies as soil pore-water freezes. This finding is contrary to the findings of other researchers who had to introduce an arbitrary ice impedance factor to make computed and measured ice contents agree. The ice impedance factor has the effect of reducing the permeability by several orders of magnitude as the volumetric pore-ice content increases. In this study, good agreement between computed and measured ice contents was obtained without the use of an impedance factor.

To demonstrate an application of the revised model, a simulation of freezing and thawing in a soil cover system was carried out and compared to field data collected during the winter of 1993 / 1994 at a silver mine near Houston, B.C. For comparisons between the field data and simulations, the soil surface temperature beneath the snow pack had to be estimated as the numerical model does not account for heat and mass flux through snow layers. Daily infiltration during the spring thaw was also estimated based on averaged meteorological data provided by Equity Mine. 


\section{ACKNOWLEDGMENTS}

I am grateful for the assistance I received from many people during my time as a graduate student. I would like to thank the laboratory technician, Alex Kozlow, and the secretaries Lynn Bantle, Heather Larson and Debbie Pfeifer for helping me with various tasks over the past two years and for being friendly and outgoing. I am grateful for the help I received from Jamshid Yazdani in the geotechnical laboratory. I owe a debt of gratitude to Mike O'kane, a project engineer in the Unsaturated Soils Group, for helping introduce me to the world of geotechnical engineering and for being a good friend. I also thank Placer Dome Canada Inc. for providing the funding necessary to carry out this research.

I would like to thank my supervisor, Professor Ward Wilson, for giving me this project and for encouraging me to carry on when the going was tough. I am also grateful for the advice I received from Professors Lee Barbour and Del Fredlund, and from the members of my examining committee: Professors Don Gray, Bob Besant, and Dennis Pufahl.

I deeply appreciate the love and support I have received throughout my life from my parents, Paul and Edith Newman. Finally, I will be eternally grateful to my wife and colleague, Lori. She sacrificed spending time with our newborn son, Ben, to return as a full time funded graduate student so that we could both afford this education. 


\section{TABLE OF CONTENTS}

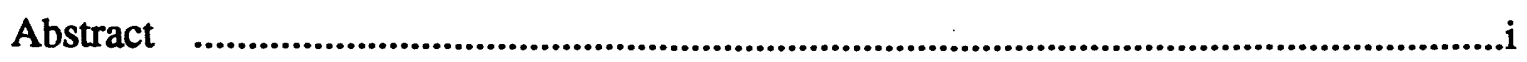

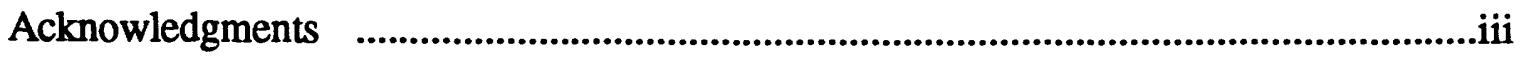

Table of Contents

List of Tables

List of Figures .................................................................................................................viii

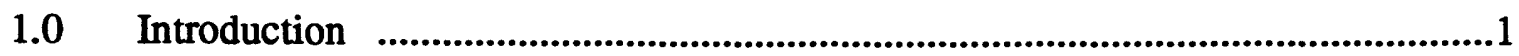

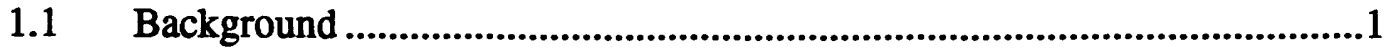

1.2 Objectives of Research Program ...............................................................2

1.3 Organization of Thesis ...........................................................................3

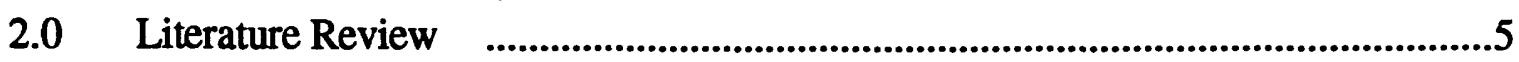

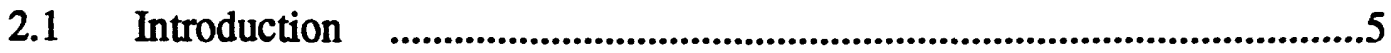

2.2 The Physical Processes Occurring During Soil Freezing ..............................7

2.2.1 Moisture Transfer Mechanisms …..............................................8

2.2.1.1 Vapour Transfer .............................................................8

2.2.1.2 Liquid Transfer ................................................................10

2.2.1.3 Moisture Zones in Frozen Soils .....................................11

2.2.2 Heat Transfer Mechanisms ..................................................12

2.2.2.1 The Relevance of Convective Heat Transfer ..................13

2.3 Unfrozen Water in Frozen Soils ............................................................14

2.3.1 Thermodynamic Equilibrium …....................................................17

2.3.2 Relating Soil Freezing Curves to Soil Water Characteristic

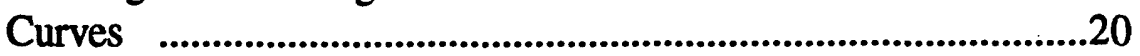

2.4 Thermal and Hydraulic Properties of Frozen Soils ..................................29

2.4.1 Thermal Conductivity ..............................................................29

2.4.2 Volumetric Specific Heat and Apparent Specific Heat ...................33

2.4.3 Water Coefficient of Permeability ...............................................35

2.5 Numerical Models of Freezing/Thawing Soils ...........................................38

2.5.1 Hydrodynamic Soil Freezing Models ...........................................40

2.6 Summary ..........................................................................................42 


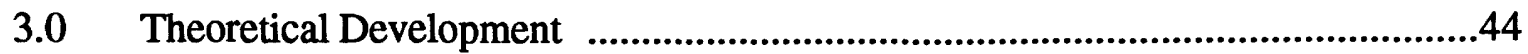

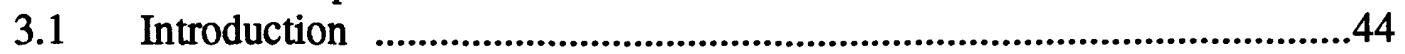

3.2 Existing Heat and Mass Transfer Equations ...........................................45

3.3 Derivation of the Modified Heat and Mass Transfer Equations for Freezing Soils ......................................................................................47

$3.4 \quad$ Solution Strategy $\quad$.................................................................................52

4.0 Revised Model Verification Program ..........................................................55

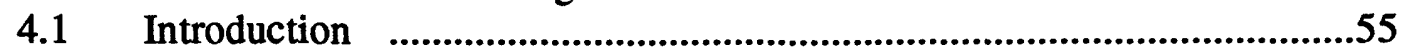

4.2 Laboratory Data Modelling Program …................................................55

4.3 Soil Properties Used in the Laboratory Data Modelling Program ............61

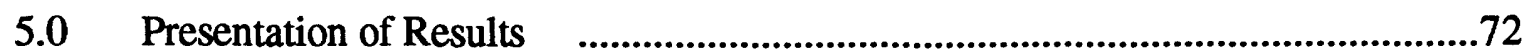

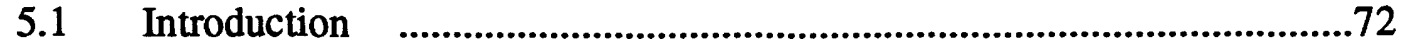

5.2 Results of Modelling Jame (1980) Laboratory Data ...............................72

5.3 Calibration of Hydraulic Properties ........................................................78

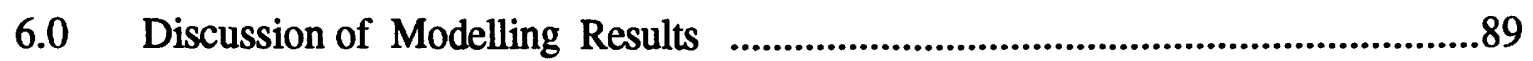

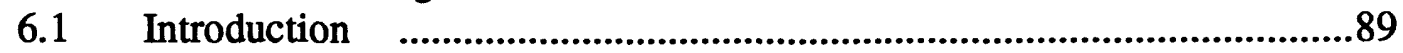

6.2 Advantages and Limitations of the Revised Numerical Solution ...........89

6.3 The Laboratory Data Modelling Program ..............................................92

6.3.1 General Comments Regarding the Simulated

Temperature and Moisture Profiles ............................................92

6.3.2 Reasons for the Discrepancy Between Computed

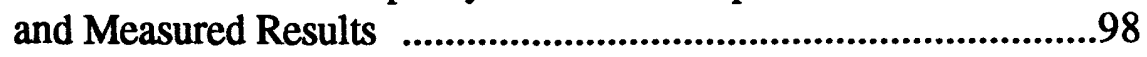

6.4 The Sensitivity of Computed Results to Soil Property Functions ............101

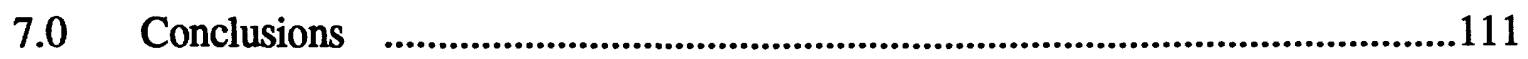

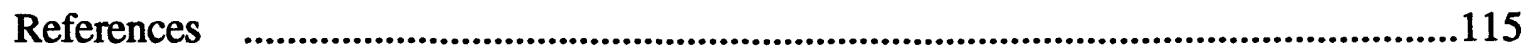

Appendix A: Field Data Modelling Application …....................................................121

A1 Introduction to Field Data Modelling .....................................................121

A2 Soil Properties Used in the Field Data Modelling ..................................122

A3 Modelling Equity Mine Field Data ............................................................127

Appendix B: Subroutine Call Out Diagram ..................................................................135

Appendix C: Main Program Code $\quad$.............................................................................137

Appendix D: Subroutine Code …........................................................................142 
Appendix E: Subfunction Code 


\section{LIST OF TABLES}

Table 2.1 Unfrozen Water Content Parameters For Use In Equation 2.9 ...............28

Table 4.1 Test Conditions for Jame (1980) Experiments

Table 5.1 Summary of Test Conditions Used in Calibration of Hydraulic Properties . .79 


\section{LIST OF FIGURES}

Figure 2.1 Definition of Various Zones in a Frozen Soil Based on Miller (1972) and Harlan (1973)

Figure 2.2 Temperature and Unfrozen Water Content of Silt and Clay

for Various Initial Water Contents (after Yong, 1965) 15

Figure 2.3 Unfrozen Water Contents in Typical Nonsaline Soils (after Nersesova and Tsytovich, 1965)

Figure 2.4 Relationship Between Freezing Point Depression and Water Content, and Between Unfrozen Water Content and Negative Temperature (after Jame, 1972)

Figure 2.5 Theoretical Relationship Between Matric Suction and Negative Temperature for a Coarse Grained Soil (after Jame, 1972)

Figure 2.6 Schematic of Partially Frozen Soil Showing Relevant Stress

State Variables (After Miller 1973)

Figure 2.7 Experimental Relationships Between Soil Freezing Curves and Soil Water Characteristic Curves Including Hysteresis Effects (after Koopmans and Miller, 1966) .22

Figure 2.8 Combination of Experimental and Theoretical Soil Water Characteristic and Soil Freezing Curves for Windsor Sandy Loam (after Black and Tice, 1989) .24

Figure 2.9 Comparison of Experimental and De Vries Method Thermal Conductivity for Non-Freezing Case (after Jame, 1977) 33

Figure 2.10 Hydraulic Conductivity of Frozen Soils (after Nixon, 1992) 37

Figure 3.1 Flowchart Showing Criteria for Using Modified Soil Freezing Equation During Assembly of Element Stiffness and Mass Matrices

Figure 4.1 Experimental Results for Test 1 (after Jame, 1980) ................................58

Figure 4.2 Experimental Results for Test 2 (after Jame, 1980) ..................................59 
Figure 4.3 Experimental Results for Test 3 (after Jame, 1980) .60

Figure 4.4 Grain Size Distribution for Silica Flour Soil Property Testing .61

Figure 4.5 Soil Freezing Curve for Silica Flour (after Jame, 1972)............................62

Figure 4.6 Semi-Log Plot of Soil Freezing Curve for Silica Flour .63

Figure 4.7 Theoretical and Experimental Soil Water Characteristic Curves (Experimental Data Was Approximated at Suctions Above $100 \mathrm{kPa}$ ) .65

Figure 4.8 Ratio "G" for Change in Matric Suction and Change in Negative Temperature as a Function of Volumetric Water Content for Silica Flour in the Frozen Zone .65

Figure 4.9 Relative Coefficient of Permeability for Silica Flour .66

Figure 4.10 Coefficient of Permeability - Ice Impedance Factor .................................68

Figure 4.11 Thermal Conductivity for Unfrozen Silica Flour …..................................69

Figure 4.12 Thermal Conductivity for Frozen Silica Flour (for different initial gravimetric water contents and neglecting mass transfer during freezing) . .70

Figure 4.13 Volumetric Specific Heat in Unfrozen Soil . .71

Figure 5.1 Cold End Temperature Boundary Conditions for Tests 1-3 (Jame, 1980) .74

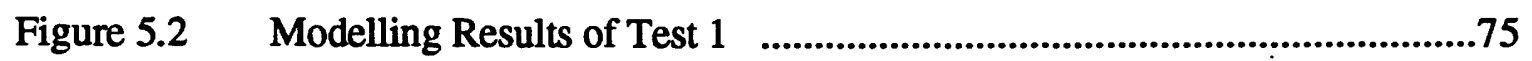

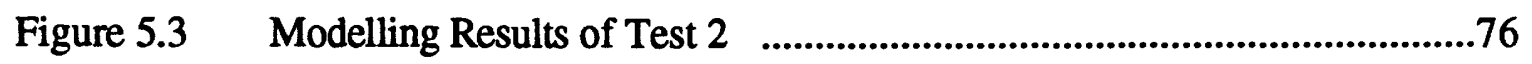

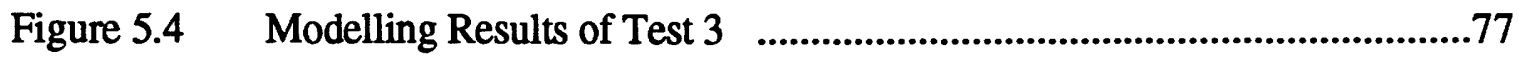

Figure 5.5a Soil Water Characteristic Curves Used in Calibration $\quad$..............................80

Figure 5.5b Relative Coefficient of Permeability Curves Obtained Using the Fredlund et al (1994) Equations for the SWC Curves Used in Calibrations

Figure 5.6 Range of Ice Impedance Factors and Relative Magnitude Applied in this Study 
Figure 5.7 Comparison of Temperature Profiles for Test 3 Using Three

Different Soil Water Characteristic Curves, ksat $=4.5 \times 10^{-5} \mathrm{~cm} / \mathrm{s}$,

and No Impedance Factor

Figure 5.8 Comparison of Moisture Profiles for Test 3 Using Three

Different Soil Water Characteristic Curves, ksat $=4.5 \times 10^{-5} \mathrm{~cm} / \mathrm{s}$, and No Impedance Factor

Figure 5.9 Comparison of Temperature Profiles for Test 3 Using Three Different

Saturated Coefficients of Permeability, SWC 3, and No Impedance

Factor

Figure 5.10 Comparison of Moisture Profiles for Test 3 Using Three Different

Saturated Coefficients of Permeability, SWC 3, and No Impedance

Factor

Figure 5.11 Comparison of Temperature Profiles for Test 3 Using Three

Different Ice Impedance Factor Coefficients, SWC 3, and

ksat $=7.0 \times 10^{-5} \mathrm{~cm} / \mathrm{s}$

Figure 5.12 Comparison of Moisture Profiles for Test 3 Using Three

Different Ice Impedance Factor Coefficients, SWC 3, and

ksat $=7.0 \times 10^{-5} \mathrm{~cm} / \mathrm{s}$

Figure 6.1 Modelling Results of Test 1 .93

Figure 6.2 Modelling Results of Test 2 .94

Figure 6.3 Modelling Results of Test 3 .95

Figure 6.4 Problems with Numerical Gauss Point Suction Estimations 100

Figure 6.5 Soil Water Characteristic Curve Used in Sensitivity Study 101

Figure 6.6 Moisture Content Profiles Computed Using Three Different Soil Water Characteristic Curves

Figure 6.7 Moisture Content Profiles Computed Using Three Different Saturated Coefficients of Permeability .104

Figure 6.8 Moisture Content Profiles Computed Using Three Different Ice Impedance Factors 106

Figure 6.9 Tornado Plot Comparing Position of Temperature Profiles 
Figure 6.10 Tornado Plot Comparing Ice Contents Computed Using Slightly Different Soil Property Functions

Figure 6.11 Tornado Plot Comparing Unfrozen Water Contents Computed Using Slightly Different Soil Property Functions

Figure 6.12 Tornado Plot Comparing Unfrozen Water Contents Computed Using Slightly Different Soil Property Functions

Figure A1 Soil Water Characteristic Curves for Modelling Field Data (after Swanson, 1995)

Figure A2 Relative Coefficients of Permeability for Modelling Field Data (modified after Swanson, 1995)

Figure A3 Soil Freezing Curve Data Used in Field Modelling Program 124

Figure A4 Ratio of Proportionality, 'G' Between Change in Suction and Change in Temperature

Figure A5 Thermoconductivity Functions of Cover Material for Non-Freezing Case (after Swanson, 1995)

Figure A6 Specific Heat Functions of Cover Material for Non-Freezing Case (after Swanson, 1995)

Figure A7 Mean Daily Air Temperatures Over Winter of 1993/1994 at Weather Station On Top of Main Waste Rock Dump 128

Figure A8 Measured Soil Temperatures from Equity Mine at Three Depths (from O’kane, 1995) 129

Figure A9 Computed and Measured Soil Temperatures at Two Depths 131

Figure A10 Computed Gravimetric Ice Contents at $5 \mathrm{~cm}$ Depth During Field Data Simulation

Figure A11 Computed Soil Thermal Conductivity Values at $5 \mathrm{~cm}$ Depth During Field Data Simulation .132

Figure A12 Computed Liquid Water Contents at Two Depths During Field Data Simulations 


\section{CHAPTER 1 INTRODUCTION}

\subsection{Background}

Researchers have been interested in analysing freezing processes in soils throughout this century. In the 1970's oil companies proposed construction of chilled gas pipelines stretching from northern permafrost regions to populated areas in the south. Oil companies were concerned about the effects of frost damage on the pipelines and it was this interest, and the accompanying influx of research dollars, which facilitated means for predicting frost heave phenomenon.

Early research focused exclusively on thermal processes in freezing soils, but in the 1970's it became clear that freezing and thawing analyses must include both heat flow and mass transfer. Throughout the 1970's and 1980's researchers developed numerous heat flow and mass transfer models for freezing soils. While engineers were interested in frost heave predictions, soil scientists focused on predicting temporal winter temperature and moisture content profiles in agricultural soil. Various theoretical approaches were tried with varying degrees of success. A practical model was never developed for general use by practicing engineers or soil scientists.

During this period it became clear that certain problems were common to all the proposed models. These problems related to the relationship between pore-water pressures and temperature in different soil types; the method by which the unfrozen water content 
versus temperature function was obtained; and the applicability of the water permeability versus suction (or water content) function for water in partially frozen soils. The Clapeyron equation relates a change in pressure between any two phases of a substance (i.e., liquid - vapour, or liquid - solid) to the change in temperature of the system. It has been used with limited success to couple the heat flow and mass transfer equations for freezing soils, but research has shown that the Clapeyron equation only applies in certain circumstances (i.e., if the soil water is wholly capillary or, wholly adsorptive). The Clapeyron equation has also been used as a tool to predict the amount of unfrozen water in a frozen soil as a function of temperature below freezing. Finally, it is known that the water coefficient of permeability in a partially frozen soil is reduced by pore-ice build up, but there has been disagreement about how to predict the new permeability in the frozen zones. As a result, some researchers have used an empirical impedance factor to calibrate the permeability functions in their models.

\subsection{Objectives of Research Program}

The two primary objectives of this research program are as follows:

1. to develop unsaturated soil freezing theory from a geotechnical engineering perspective, and

2. to devise a numerical technique for verifying this theory by modifying an existing nonfreezing soil heat flow and mass transfer model.

In this study, the existing numerical model SoilCover (MEND, 1993) was modified to verify the theory and numerical solution technique. The non-freezing SoilCover (MEND, 1993) model was developed as a design tool for engineers working on soil cover systems over acid generating mine waste rock and tailings. SoilCover (MEND, 1993) uses Darcy's and Fick's laws to describe the flow of liquid water and vapour in the soil below the soil - atmosphere boundary. A modified Penman formulation (Wilson, 1990) computes the actual evaporation from the soil surface and thus couples the soil model with the atmosphere. The unmodified version of SoilCover (MEND, 1993) is not 
capable of modelling soil temperature and suction profiles if the ground surface temperature drops to $0^{\circ} \mathrm{C}$ or colder. Therefore, long term predictive modelling is difficult because there is no continuity between summer and winter seasons.

\subsection{Organization of Thesis}

Problems associated with freezing soil analyses are discussed in detail in the literature review chapter (i.e., Chapter 2). Chapter 2 also discusses the mechanisms of heat flow and mass transfer in freezing and non-freezing soils, and the methods by which the soil thermal and hydraulic properties can be computed. Heat flow and mass transfer processes in unsaturated soils are fairly well understood for the non-freezing case, but analysis of the phenomenon is more complex if part of the pore space is occupied by ice. Where most of the soil thermal and hydraulic properties are functions of changing water contents in the unfrozen case, they become functions of changing water and changing ice contents if the soil is frozen. A brief discussion of the various types of soil freezing models which have been developed in the last twenty years is also presented in Chapter 2.

The theoretical development chapter of this thesis (i.e., Chapter 3) presents the coupled heat flow and mass transfer equations used in the non-freezing version of SoilCover (MEND, 1993). Following this, the modified heat flow and mass transfer equations which include phase change phenomenon are derived. The modified theory is uniquely incorporated into the existing model in such a way that it is consistent with the model's finite element method formulation.

Chapter 4 introduces the laboratory data model verification program. Initial verification of the revised model was carried out using laboratory freezing data obtained by Jame (1977) at the University of Saskatchewan. During the laboratory data modelling program, the sensitivity of computed moisture and temperature profiles to slight changes in soil hydraulic properties was tested. 
In Chapter 5, the results of the laboratory verification program are presented. Chapter 6 discusses the modelling results and probable reasons for any discrepancy between computed and measured results are given. In addition, general comments about the advantages and limitations of the modified soil freezing model are presented. Concluding comments are given in Chapter 7.

Appendix A presents a field application of the revised model and compares simulation results with freezing and thawing data collected in the field. The field data was obtained by O'kane (1995) from an instrumented clay - till cover over mine waste rock at a silver mine, near the town of Houston, in the interior of British Columbia. Appendices B through $\mathrm{G}$ include the revised computed code, support files, and sample input files usd in the field data modelling. 


\section{CHAPTER 2 \\ LITERATURE REVIEW}

\subsection{Introduction}

From a historical perspective, the conceptualizations of, and subsequent attempts at analyzing freezing and thawing processes in soil dates back to lectures given by Stefan and Neumann in the late 1800's (Jumikis, 1966). They presented exact solutions to predict the depth of frost penetration as a function of time. Their methods were based on questionable assumptions about the freezing temperature of pore-water, the shape of the temperature profile below the frost line, the thermal properties of soil at temperatures above and below freezing, and the significance of mass transfer mechanisms. In addition, their solutions were given for a unique set of boundary conditions which seldom exist in nature.

Relatively little research related to soil freezing was carried out in the first half of this century. Bouyoucos (1920) demonstrated that a certain amount of unfrozen water exists in frozen soils. He also concluded that the unfrozen water exists because of interactions between the mineral matrix and pore-water. In 1929, Taber showed that a major cause of volume changes in frozen soil was the formation of segregated ice. While researchers were aware that pore-water in soils freezes at temperatures below $0^{\circ} \mathrm{C}$, it $\cdot$ was Fisher (1924) who presented a detailed discussion on the freezing of water in capillary systems which indicated the dominant influence on freezing point depression was not salt concentration, but the forces by which water was held in the soil pores. Schofield (1935) 
presented an underived freezing point depression calculation as a function of negative pore-water pressure, and in 1935, Beskow demonstrated that negative pore-water pressures also develop within a fine-grained soil during freezing.

In the post World War II era a large increase in freeze/thaw and permafrost research was initiated. Large scale engineering projects throughout the world required an accurate means for predicting freeze / thaw behaviour and its effect on engineered structures. Kemper (1960) showed that water transfer in unsaturated soils takes place in thin liquid films which exist between adjacent soil particles. Dirksen and Miller (1966) showed that the water transfer is altered by the presence of pore-ice, and Jumikis (1966), Hoekstra (1966) and others, showed that freezing and thawing in soils involves significant mass transfer processes in addition to the more obvious heat transfers.

During the late 1960's and throughout the 1970's a considerable amount of research was carried out for freezing and thawing soils. During this period there was little consensus among researchers about how to analyse the physical processes occurring in a freezing soil. As a result, numerous theories were proposed which attempted to describe the physics of soil freezing. Numerous models were also developed which attempted to provide engineers and soil scientists with a means of mathematically analysing freeze I thaw behaviour. Works by Harlan (1973), Guymon and Luthin (1974), Jame (1977), Konrad and Morgenstern (1990), Nixon (1992) and others have tried to present mechanistic models for freezing behavior in fine grained soils with or without frost heave. Flerchinger (1989) presents a simultaneous heat and water model for a snow-residue-soil system using conventional heat flow and mass balance theory. His model includes osmotic effects, but neglects frost heave.

The bulk of this literature review will focus on research conducted from the mid 1960's to the present. This research has, in general, dealt with the following:

understanding the physical processes which take place during freezing, 
- $\quad$ quantifying the amount of unfrozen water in frozen soils as a function of temperature or suction,

- determining the thermal and hydraulic properties of unsaturated soils subjected to freezing and thawing for use in analytical models, and developing numerical models to analyse the coupled heat and mass transfer processes.

These will be discussed in turn.

\subsection{The Physical Processes Occurring During Soil Freezing}

A soil system consists of a mineral matrix whose voids may contain air, water, watervapour, ice, and various solute solutions. The soil itself is physically discontinuous from the atmosphere at its surface, so it is common when analyzing soil behaviour to define the surface as a natural boundary. Although the soil appears discontinuous with the atmosphere at this point, it is the energy and mass fluxes across the soil - atmosphere boundary which cause changes in the stress states of the soil.

When the temperature at the soil surface drops, a thermal gradient develops between the cold surface and the warmer soils below. A transient heat flow is initiated which includes conductive, convective, and radiative heat transfers. If the boundary temperature drops below the freezing point of the pore-water, some of the pore-water will freeze and release latent heat into the system. As the pore-water freezes the amount of water remaining in the liquid state is reduced and negative pore-water pressures (or suctions) are induced, or increase in the case of an initially unsaturated soil. In response to the pressure and temperature gradient, moisture, if available, flows in its liquid and vapour phase from lower regions in the soil to and beyond the advancing freezing front, where it freezes and releases more latent heat. If the conditions are right (i.e., soil type, overburden pressure, water content etc.), ice lenses may form and result in heave of the soil surface. The frost front advance continues until the frozen zone can no longer remove all the latent energy 
released by the phase change at the frost front. Liquid flux will continue throughout the system as long as the water permeability is sufficiently high or until the pores become completely blocked by ice, at which point liquid flux will be shut down behind the frost front.

Most researchers today would agree that the previous paragraph describes the basic processes which take place during soil freezing. The problem with this description of the physical processes is that it is too simplified. A rigorous analysis of this type of coupled heat and mass transfer problem requires a deeper understanding of the individual physical processes and their significance on the final analytical solutions.

\subsubsection{Moisture Transfer Mechanisms}

As discussed in the previous section, moisture transfer in freezing / thawing soils can occur in the liquid or vapour phase. The type of liquid transfer depends on the soil geometry, degree of saturation, and availability of water. While researchers have been aware of vapour transfer processes in non-freezing soils for the past 50 years (Smith, 1939), the significance of vapour transfer in freezing soils subjected to different types of boundary conditions is still under debate.

\subsubsection{Vapour Transfer}

The driving force for vapour transfer is the gradient between the partial vapour pressure of pore-water in the warmer soils at depth and the partial vapour pressure of pore-water in the upper regions of soil, just below the deepest point of ice formation. Thus, vapour transfer takes place towards the colder regions, or along the drop of the thermal gradient. Jumikis (1966) mentions the importance of vapour transport in soils with relatively large void sizes, especially in the case where there is no continuous liquid phase. $\mathrm{He}$ acknowledges that vapour diffusion also takes place in soils with particles coated by moisture films, but he adds that it is very difficult to analyse this type of diffusion due to the difficulty in expressing the geometry of the voids and surface topography of the soil particles. 
The relevance of vapour transfer in well graded soils is considered by Gray et al., (1985). While studying over-winter soil moisture changes, Gray observed post-winter field moisture conditions which suggested a significant amount of vapour transfer had occurred. By comparing the energy and mass balances between two different sites on the Canadian Prairies, Gray was able to back calculate what appeared to be a substantial vapour transfer event.

Harlan (1973) was one of the first investigators to model coupled heat and mass transfer in freezing soils. His model was developed on the assumption that vapour transfer has a negligible effect on the net mass transfer. The assumption made by Harlan (1973) may have some validity for the tests he conducted using Yoho Clay soil (with relatively low porosity), but his modelling of freezing in Del Monte Sand most likely had error introduced by omission of vapour transport. Harlan (1973) was not able to present a comparison of laboratory data and analytical results so it is hard to make quantitative comments about the validity of his assumptions. Many subsequent researchers followed Harlan's lead and omitted the vapour transport mechanism. in their freezing models.

Philip and de Vries (1957) presented heat and mass transfer equations for porous materials which included vapour transfer under non-freezing conditions. Their approach was limited from a geotechnical engineering perspective. They assumed volume change did not occur, they neglected liquid flow resulting from changes in total stress, and they assumed liquid flow was in response to changes in volumetric water content, and not hydraulic head.

Dakshanamurthy and Fredlund (1981) presented un-coupled simultaneous heat and mass transfer equations which included air, water, vapour and heat flow in non-freezing, unsaturated swelling soils. Wilson (1990) used simplified forms of these relationships and presented two coupled heat and mass transfer partial differential equations with hydraulic head and temperature as dependent variables. Wilson's equations are the basis for this research program which expands the heat and mass transfer model to include freezing and 
thawing in soils. Wilson's (1990) coupled heat and mass transfer equations are discussed in detail in Chapter 3.

\subsubsection{Liquid Transfer}

Soil physicists describe the transport of water through soils as bulk flow or film-capillary flow (Jumikis, 1966). In a saturated soil, all of the voids are filled with water and the flow is considered bulk fluid flow. In unsaturated soils, water is transported by filmcapillary flow mechanisms. In either case, and for saturated or unsaturated conditions, water flows in response to changes in hydraulic head.

The flow of liquid water through saturated soils is commonly described using Darcy's law, where the rate of flow through a soil mass is proportional to the hydraulic head gradient. The coefficient of proportionality between the flow rate and the hydraulic head gradient is called the coefficient of permeability. This coefficient is relatively constant for any specific saturated soil. Darcy's law also applies to flow of water through unsaturated soils. In this case the coefficient of permeability is not constant, but a function of water content or matric suction. Matric suction is defined as the difference between pore-air and porewater pressure (Fredlund and Rhardjo, 1993). The coefficient of permeability is generally a function of any two of the following three volume-mass soil properties: the degree of saturation, the void ratio or the water content (Fredlund and Rhardjo, 1993).

Water can be considered to flow only through pores that contain water. As a result, the air filled pores are non-conductive channels to the flow of water (Fredlund and Rhardjo, 1993). Childs (1969) speculated that the air-filled pores in an unsaturated soil behave similarly to the solids which make up the soil. This is similar to the assumption made by Harlan (1973) that the suction - permeability relationship of a partially frozen soil is the same as that of the unfrozen, unsaturated soil because the frozen water is treated as part of the solid matrix of the soil. Experiments have been conducted to verify Darcy's law for water flow through unsaturated soils, but experimental data supporting the Harlan (1973) 
assumption has been lacking. Results of numerical modelling by Jame (1977), Taylor and Luthin (1978) and others did not verify the Harlan (1973) hypothesis. However, results of this current study suggest his assumptions may have some validity. This will be discussed in section 2.4.3 and in Chapter 6.

\subsubsection{Moisture Zones in Frozen Soils}

During the 1960's, Jumikis (1966), Dirksen and Miller (1966), Hoekstra (1966) and others imagined that a freezing soil consisted of three zones: a frozen zone, a frost front, and unfrozen soil. The frost front is the point of farthest frost advance into the soil. Miller (1972) first made reference to a thin zone of low permeability frozen soil which lies between an ice lens (if present) and the unfrozen soil called the frozen fringe. Harlan (1973) makes reference to an additional zone "in close proximity to the freezing front" where a large redistribution of water takes place. Figure 2.1 illustrates these zones.

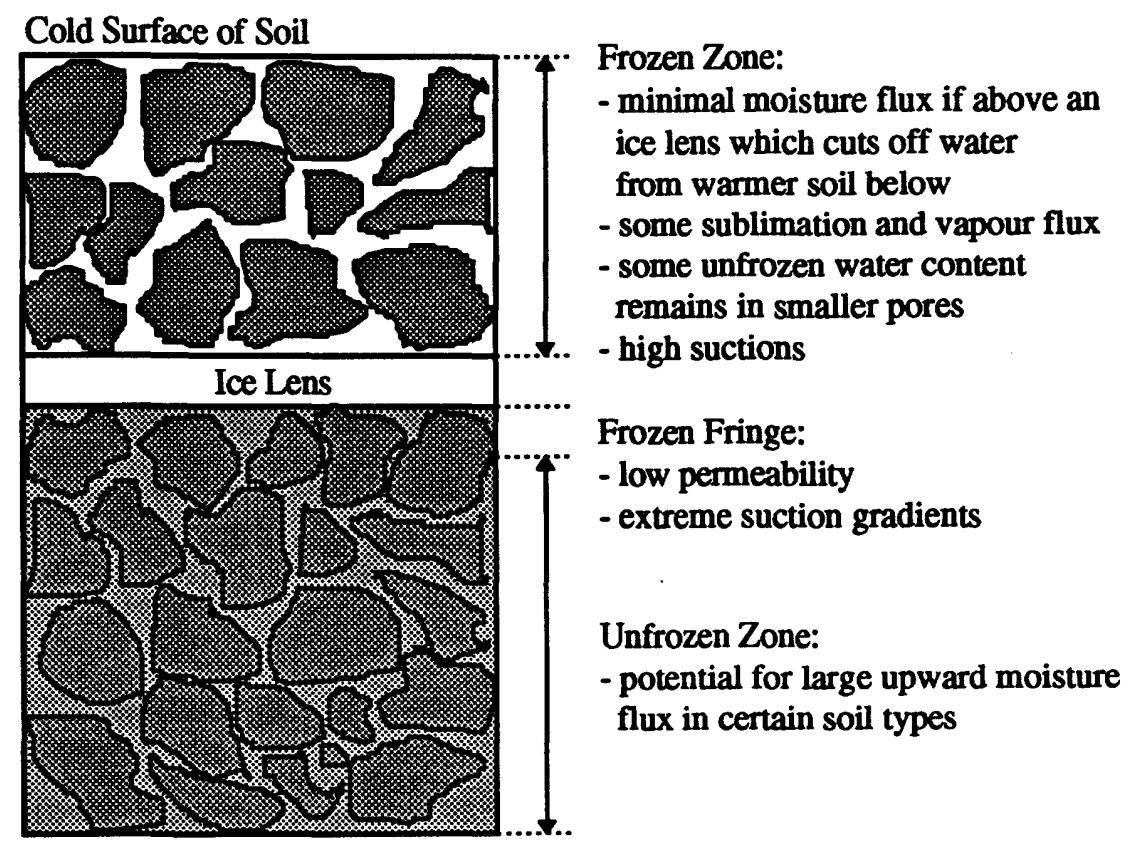

Figure 2.1 Definition of Various Zones in a Frozen Soil Based on Miller (1972) and Harlan (1973) 
Ice lenses are thin plates of ice segregated from the soil matrix. The process by which they are formed has been the topic of great debate over the years. Ice lensing results in frost heave and considerable damage to surface structures. Ice lensing and heaving was initially studied by Taber (1929) and Beskow (1935) earlier in the century. More recently, Miller (1978), Konrad and Morgenstern (1980) and Nixon (1991) discuss the process in some detail and how it can be analysed. Ice lensing is significant because it can cause damage to surface structures and because it affects the moisture flow conditions in the frozen soil above the lens. It is generally agreed that during freezing, liquid moisture flux in the frozen zone above an ice lens is negligible because the ice lens acts as a barrier to liquid flow from below. Ice lensing is not considered in the computer model developed in Chapter 3.

Dirksen and Miller (1966) and Hoekstra (1966) conducted experiments which revealed a large moisture flux from the unfrozen soil to the freezing front. They also noted that the ice content behind the frost front increased with time. In order to explain this phenomenon, vapour transfer analyses were conducted. From these studies, it became evident that vapour flow alone could not explain the quantity of water in the frozen soil. Hoekstra (1966) observed that, for his test conditions, the magnitude of moisture flow in the frozen and unfrozen zones were similar. The significance of this finding is that it becomes evident liquid flow may continue in the frozen zone as long as a liquid source is available or until the permeability becomes sufficiently low to effectively prevent further moisture movement (Konrad and Morgenstern, 1980).

\subsubsection{Heat Transfer Mechanisms}

The three mechanisms for heat flux in soils are conduction, convection, and radiation. Latent heat of phase change introduces sources or sinks for heat flux. Heat conduction occurs through the soil particles, pore-water, ice, vapour, and air. In air and vapour it occurs by a process of collision between molecules and subsequent increase in kinetic 
energy. A similar process occurs in water, but additional heat is transferred through making and breaking hydrogen bonds. In the soil solid and in ice, conduction is the most efficient form of heat transfer as energy is transferred through vibration of adjacent atoms in the solid lattice structure.

Convection occurs both by molecular motion (diffusion) and heat transfer through bulk fluid flow (advection). Free convection is a mass transport phenomenon which results from density gradients that are often accompanied by temperature gradients. Thus, fluid of higher density flow towards the lower density fluid, advecting heat energy as it moves. Forced convection results from pressure differences similar to those present in groundwater.

Thermal radiation is energy emitted by matter that is at a finite temperature. It is transported by electromagnetic waves and does not require the presence of a material medium. Heat transfer by radiation in the soil pores is a function of temperature, pore geometry and water content. The effect of this type of heat transfer decreases rapidly with decreasing void size, increasing water content, and decreasing temperature (Lunardini, 1991). Thus, if radiation is only relevant to low water content, large pore size, and high temperature soils, then it can be neglected in the case of freezing soils.

Latent heat is released or absorbed when water changes phase. The latent heat of fusion (i.e., water to ice) is equal to $334 \mathrm{~kJ} / \mathrm{kg}$ of water, the latent heat of sublimation is equal to $2709 \mathrm{~kJ} / \mathrm{kg}$, and the latent heat of vapourization is $2375 \mathrm{~kJ} / \mathrm{kg}$. The latent heat input or removed from a system has a significant effect on the rate of penetration of the freezing or thawing front. Convective heat transfer is considered in the next section.

\subsubsection{The Relevance of Convective Heat Transfer}

A majority of analytical soil freezing models make the assumption that heat transfer by convection is negligible. Harlan (1973) and Guymon and Luthin (1974) included the 
convective component in their analysis, while Nixon (1975) and Taylor and Luthin (1978) found that heat transfer by convection was two to three orders of magnitude lower than that due to conduction and they omitted it. Jame and Norum (1980) used the Harlan (1973) approach without the convection term and obtained quite reasonable results modelling freezing of a fine silica flour over a period of 72 hours. Flerchinger (1987) included the convective term in his field modelling of winter freezing and thawing. Tao and Gray (1994) also included convection in their model predicting snow melt infiltration into frozen soils. It appears that the inclusion or omission of the convective term depends on the boundary conditions of the system being modeled and on the permeability of the soil. In general, convective heat transfer should be included when modelling high permeability soils, especially where there is potential for large moisture fluxes.

\subsection{Unfrozen Water in Frozen Soils}

Since Bouyoucous (1920) first showed that some part of water in a clay-water mixture remains unfrozen at temperatures as low as $-78^{\circ} \mathrm{C}$, many researchers have tried to explain, in physical and theoretical terms, the processes by which this phenomenon occurs (Williams, 1964; Williams, 1966; Nersesova and Tsytovich, 1966; Miller, 1966; Takagi, 1966; Low et al, 1968; Jame, 1972; Tice et al, 1976; Black and Tice, 1989). Since direct measurement of freezing point depression is difficult, the overall objective of their research was to develop a tool for predicting the freezing temperatures based on some easily measured soil properties. Regardless of the experimental methods used to obtain this data, it is common practice to report the freezing temperature as a function of the unfrozen water content. The converse of this relationship is the unfrozen water content versus sub-zero temperature function, which is a very useful function to have when modelling freezing in soils.

There appear to be two theories about the validity of unfrozen water content data. Nersesova (1966), Tice et al, (1966), Jame (1972) and others indicate that the unfrozen water content data for a given soil is independent of initial water content (or degree of 
saturation) and mainly a function of sub-zero temperature. Therefore, one soil freezing curve is valid for the entire range of water contents that a particular soil could have. Yong (1965), and Lange and McKim (1963), however, suggest that the unfrozen water content is dependent on both the initial degree of saturation and sub-zero temperature. Figure 2.2 shows soil freezing curves developed by Yong (1965), and Figure 2.3 shows soil freezing curves developed by Nersesova and Tsytovich (1965).

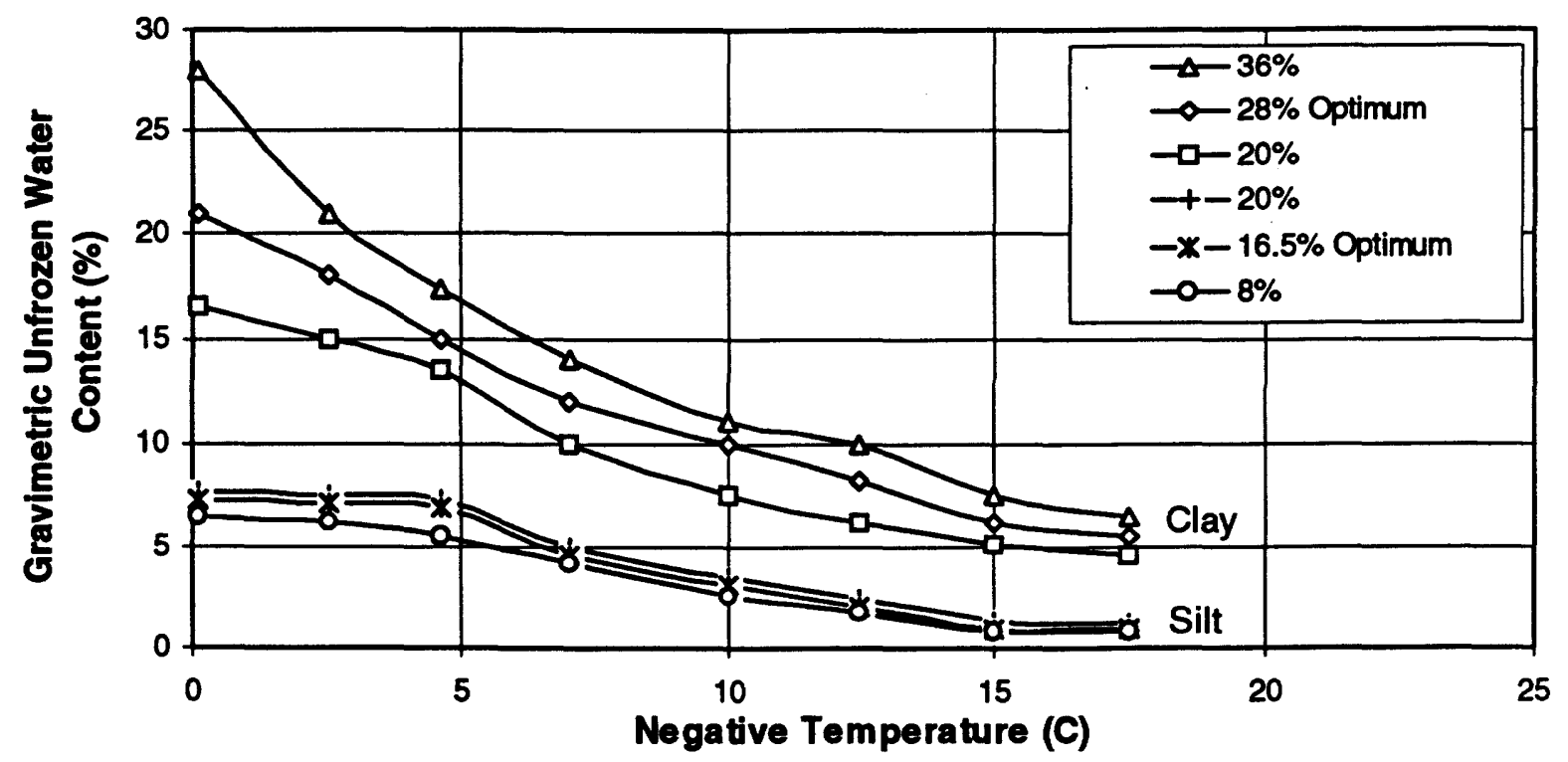

Figure 2.2 Temperature and Unfrozen Water Content of Silt and Clay for Various Initial Water Contents (after Yong, 1965)

In Figure 2.2 the experimental soil freezing curves are given for both silt and clay samples prepared at different initial moisture contents. Thus, each of the six curves in Figure 2.2 represent the soil freezing curve of a unique soil sample. Observation of Figure 2.2 reveals that samples prepared at higher water contents retain more unfrozen water at lower freezing temperatures than samples prepared at lower water contents.. In Figure 2.3, experimental soil freezing curves are given for five different materials ranging from clay to quartz sand. Although no mention is made of the initial moisture conditions (i.e., at which the samples were prepared), it is obvious that each curve is only valid for the 
specified soil type with a similar stress history. The apparent contradiction between the two figures is a result of how they are interpreted. In Figure 2.2, initial water content refers to the water content at which the sample was compacted. While in Figure 2.3, the initial water content is not the water content at which the material was compacted, but the water content present in the soil when freezing first begins. In fact, both figures show valid soil freezing curves.

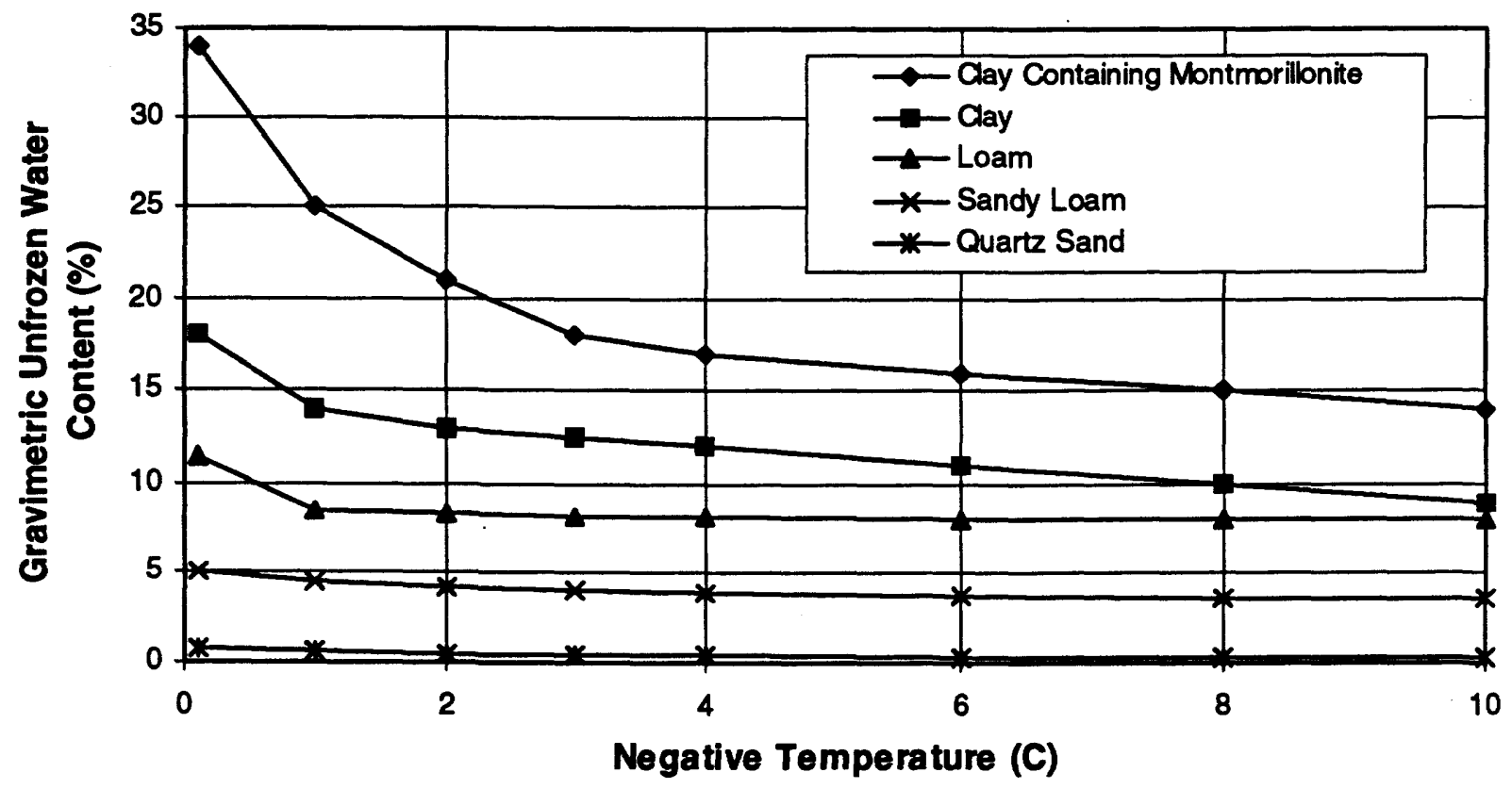

Figure 2.3 Unfrozen Water Contents in Typical Non-saline Soils (after Nersesova and Tsytovich, 1965)

Jame (1972) conducted laboratory experiments on fine silica flour to determine the relationship between unfrozen water content and freezing point depression at different freezing temperatures. One test involved gradually reducing the soil temperature until ice began to nucleate. This was done for soils at various water contents. The second test involved calorimetric determination of unfrozen water content for various sub-zero temperatures. The results of his findings are illustrated in Figure 2.4. Essentially, it was 
shown that the relationship between freezing point depression and water content is the same as the relationship between sub-zero temperature and unfrozen water content. This finding is significant because it permits the use of the freezing point depression curve for predicting unfrozen water content at different sub-zero temperatures. It will also allow correlation to be made between water content, suction (as given by soil water characteristic curves) and soil temperature. These are discussed shortly.

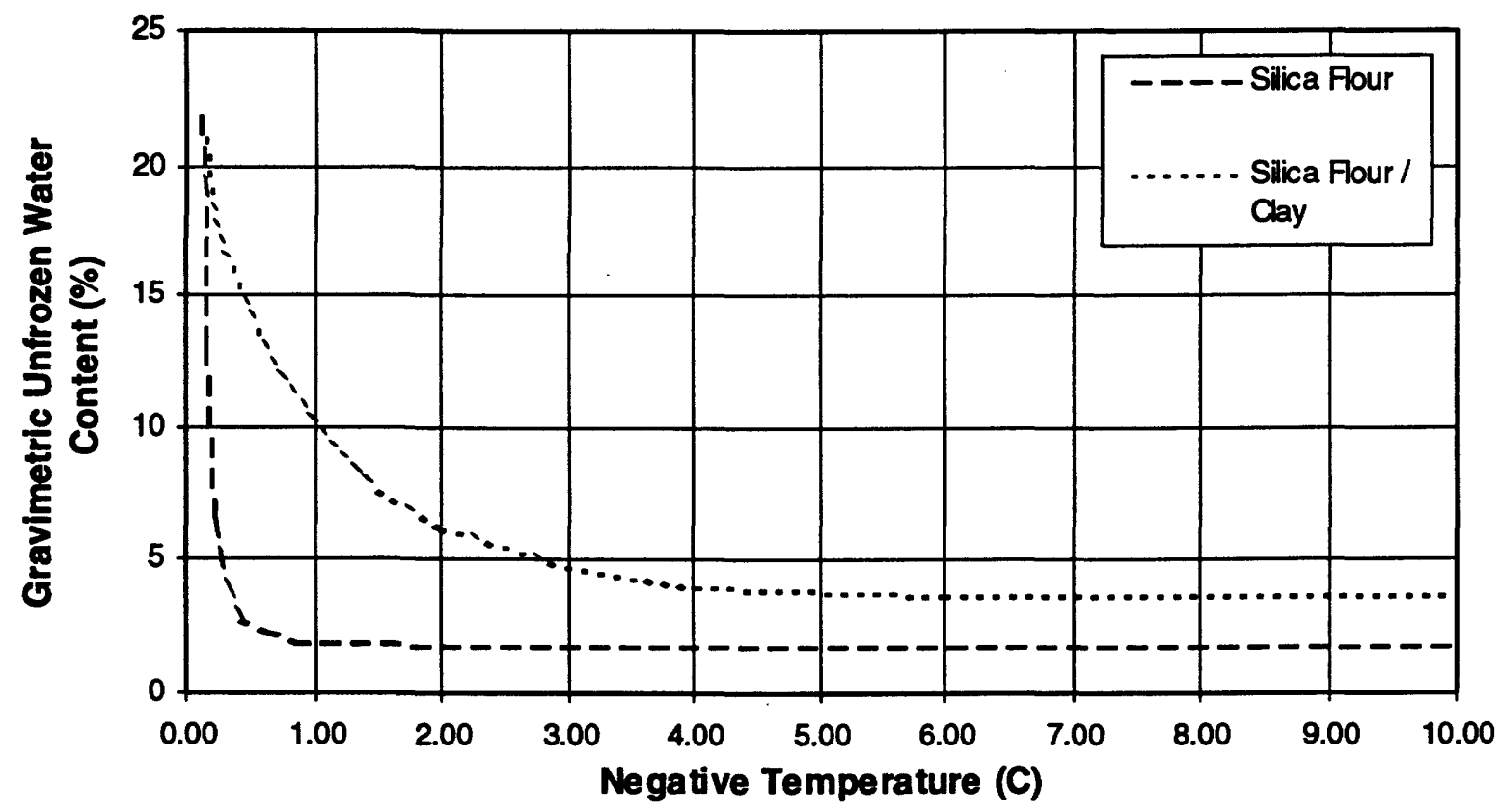

Figure 2.4 Relationship Between Freezing Point Depression and Water Content, or Between Unfrozen Water Content and Sub-zero temperature (after Jame, 1972)

\subsubsection{Thermodynamic Equilibrium}

The theoretical basis for soil water freezing relationships mentioned above comes from thermodynamic phase equilibrium theory. Therefore, it is important to discuss this theory as it applies to partially frozen, unsaturated soils. This information is also necessary for developing the computer model used in this study, as it is the basis for a correlation 
between the soil freezing curve (and freezing point depression curve) and the soil water characteristic curve.

Initial studies of unfrozen water content in frozen soils concentrated on the influence of salts on the freezing temperature of pore-water. Fisher (1924) indicated that the phenomenon is due partially to the presence of salts, and also to the way which the water is held within the soil. Fisher studied the capillary forces acting in pore-water in a freezing soil. Taber, in the 1920's, and Edlefsen and Anderson in 1943, suggested that water in fine grained soils is under the influence of adsorption forces. Miller $(1965,1973)$ distinguishes between "capillary" water forces in coarser grained, non-colloidal soils (i.e., sands and coarse silts), and "adsorptive" water forces in finer grained, wholly colloidal soils (i.e., fine silts and clays). The significance of these soil classifications becomes apparent when thermal equilibrium analysis is applied to water in frozen soils.

Analysis of the Gibbs free energy for any two phases in equilibrium can be used to derive the Clapeyron equation which relates how the equilibrium pressure changes with a change in temperature. The basic form of the Clapeyron equation is as follows:

$$
\frac{\mathrm{dP}}{\mathrm{dT}}=\frac{\Delta \mathrm{h}}{\mathrm{T} \Delta \mathrm{V}}
$$

where,

$\mathrm{P}=$ equilibrium pressure $(\mathrm{kPa})$,

$\mathrm{T}=$ temperature of the system $(\mathrm{K})$,

$\mathrm{h}=$ specific enthalpy difference between phases $(\mathrm{kJ} / \mathrm{kg})$, and

$\mathrm{V}=$ specific volume difference between phases $\left(\mathrm{m}^{3} / \mathrm{kg}\right)$.

Various forms of the Clapeyron equation have been used for different purposes in the study of freezing soils. Schofield (1935), Takagi (1963), Low et al. (1968), and Jame (1972) presented equilibrium thermodynamic relationships which related the freezing point depression of soil water to soil suction. Figure 2.5 shows the results of calculations made 
by Jame (1972) of suction as a function of sub-zero temperature for a coarse grained soil. The figure shows that very high suctions develop within a relatively small sub-zero temperature range.

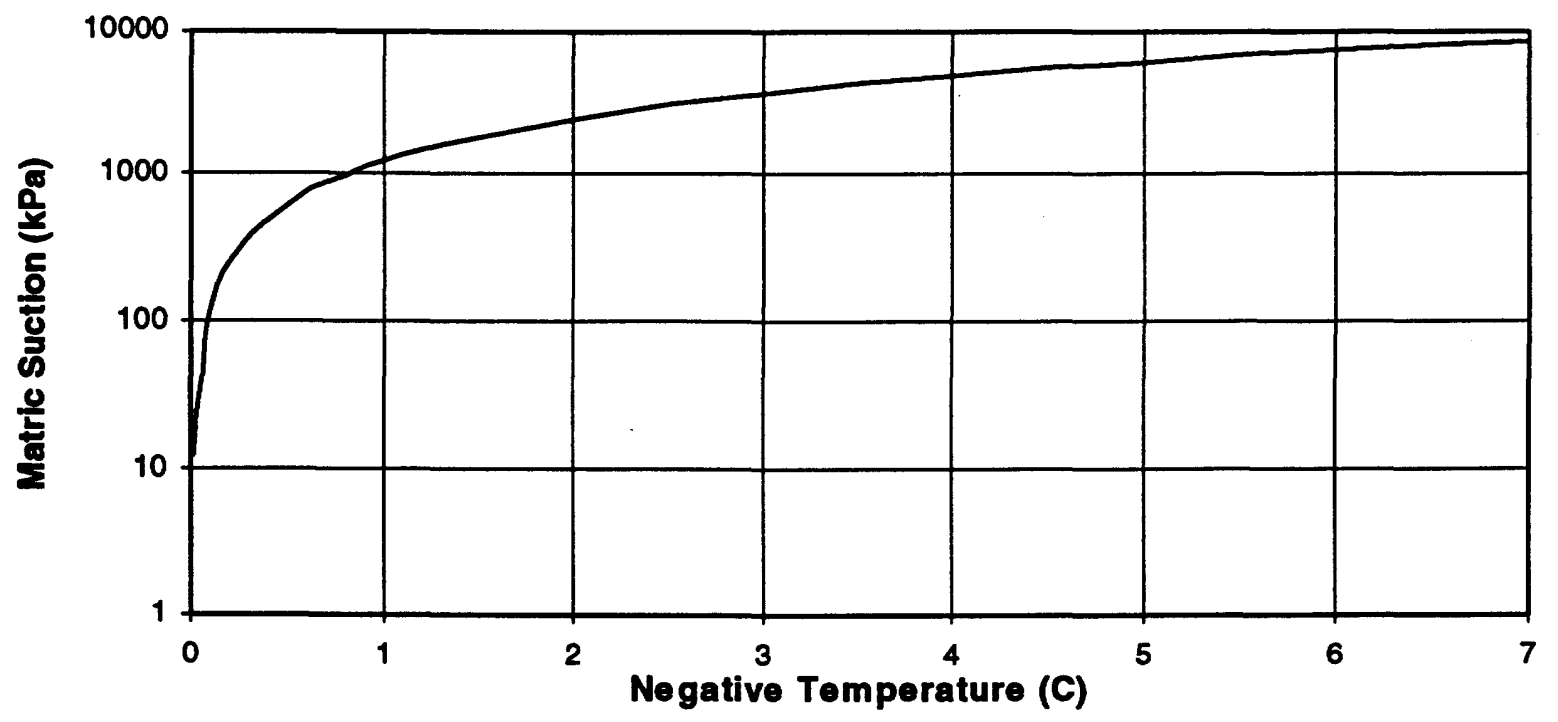

Figure 2.5 Theoretical Relationship Between Matric Suction and Sub-zero temperature for a Coarse Grained Soil (after Jame, 1972)

In the 1960's, researchers like Everett (1961), Penner (1967), and Williams (1967) used a form of the Clapeyron equation as the basis for capillary models of ice segregation and frost heave. In the 1970's and 1980's, Harlan (1973), Taylor and Luthin (1978), Jame and Norum (1980) and others presented heat and mass transfer relationships which were inherently coupled in the frozen zone by soil freezing curves and the Clapeyron equation (i.e., the soil freezing curve related water content to temperature, and the Clapeyron equation related temperature to suction for use in computing hydraulic gradients). Miller (1978), Konrad and Morgenstern (1980), and Nixon (1991, 1992) used Clapeyron type equations to relate pore-water pressures to ice pressures beneath a growing ice lens in their studies of frost heaving mechanisms. Koopmans and Miller (1966), and Black and 
Tice (1989) tried to derive the soil freezing curve from the soil water characteristic curves using equilibrium thermodynamics. The relationships between the soil water characteristic curves and soil freezing curves are discussed in the next section.

\subsubsection{Relating Soil Freezing Curves to Soil Water Characteristic Curves}

In a freezing soil, three possible phase interfaces exist: ice-water, air-water, and ice-air. If pore-ice pressure is assumed to be constant (which is apparently reasonable for normal hexagonal ice - see Jumikis, 1966; Jame 1972) and pore-air pressure is assumed constant (which is most often the case), then the two interfaces of significance are the air-water and ice-water interfaces. Figure 2.6 shows a schematic of a partially frozen unsaturated soil and the three phase stress state pressure variables. The difference between pore-air pressure and pore-water pressure in an unsaturated soil is called the matric suction. A soil water characteristic curve is used to show the variation of volumetric (liquid) water content with respect to changes in matric suction.

Williams (1964) first presented data on the relationship between a soil water characteristic curve measured at room temperature, and a soil freezing curve for the same material. The experimental data presented by Williams (1964) relating suction and temperature agreed closely with theoretical relationships given by the appropriate form of the Clapeyron equation. This finding was significant because it suggested that a theoretical soil freezing curve could be constructed using a measured soil water characteristic curve and the Clapeyron equation. In effect, the Clapeyron equation would provide the freezing temperatures corresponding to each water content or suction.

Miller (1973) related the moisture states achieved in freezing/thawing soils to drying/wetting processes in unsaturated soils. His theory was presented for soils that 
were either wholly colloidal (i.e., fine grained) or wholly non-colloidal (i.e, coarse grained). Soil types that fell between this range (i.e., were a combination of coarse and fine material) were not included in the Miller (1973) theoretical development due to the difficulty in applying the Clapeyron equation to soils dominated by both capillary and adsorptive forces. Miller found that for soils wholly dominated by adsorptive forces, a correlation exists between soil water characteristic data and soil freezing data. This agrees with the earlier experimental findings of Williams (1964), and Koopmans and Miller (1966). Results obtained by Koopmans and Miller (1966) are shown in Figure 2.7. In this figure it is clear that the freezing curve is similar to the drying curve, and the thawing curve is similar to the wetting curve. A best fit line has been added to the Figure to help with the interpretation.

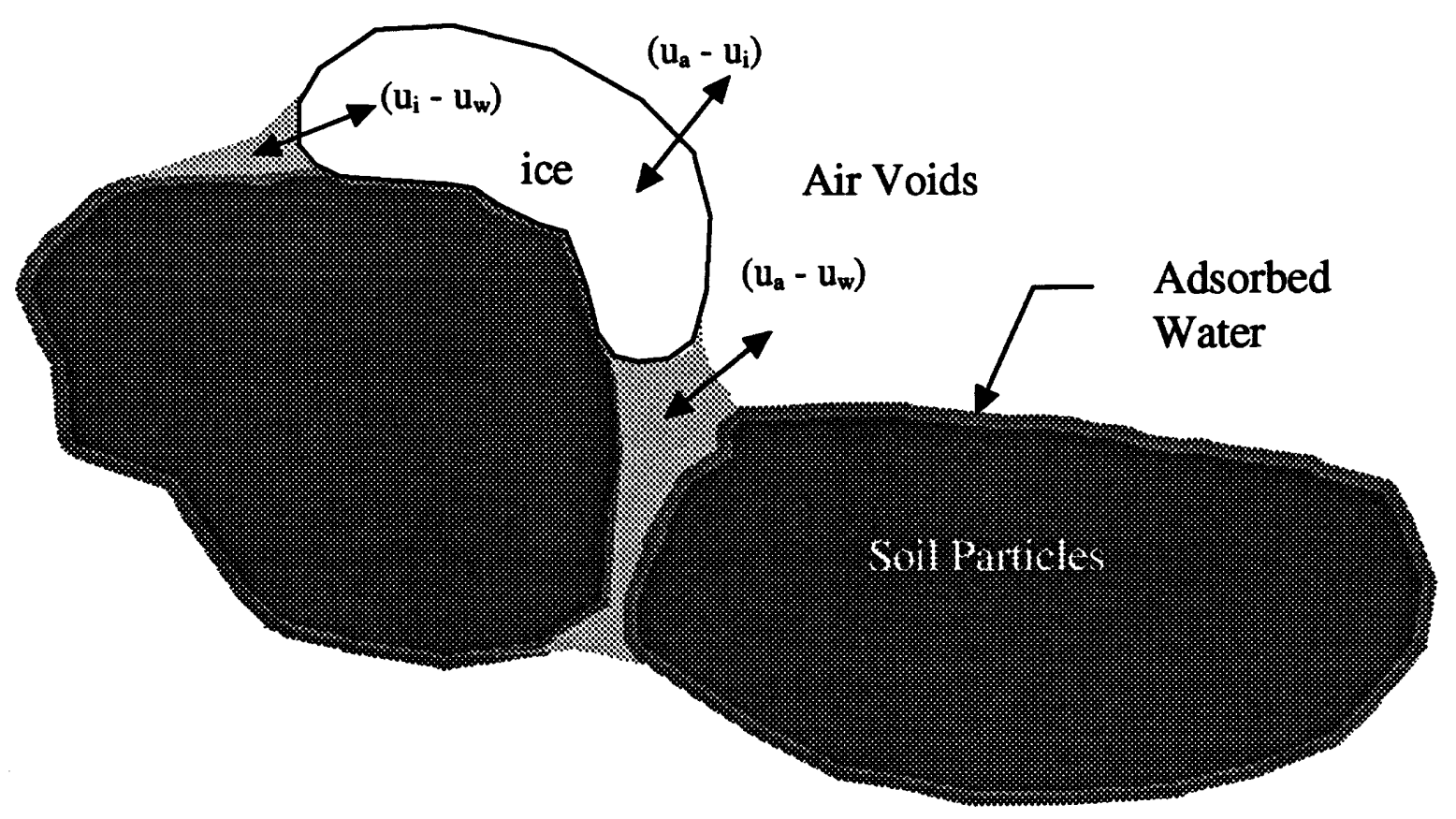

Figure 2.6 Schematic of Partially Frozen Soil Showing Relevant Stress State Variables (After Miller 1973) 


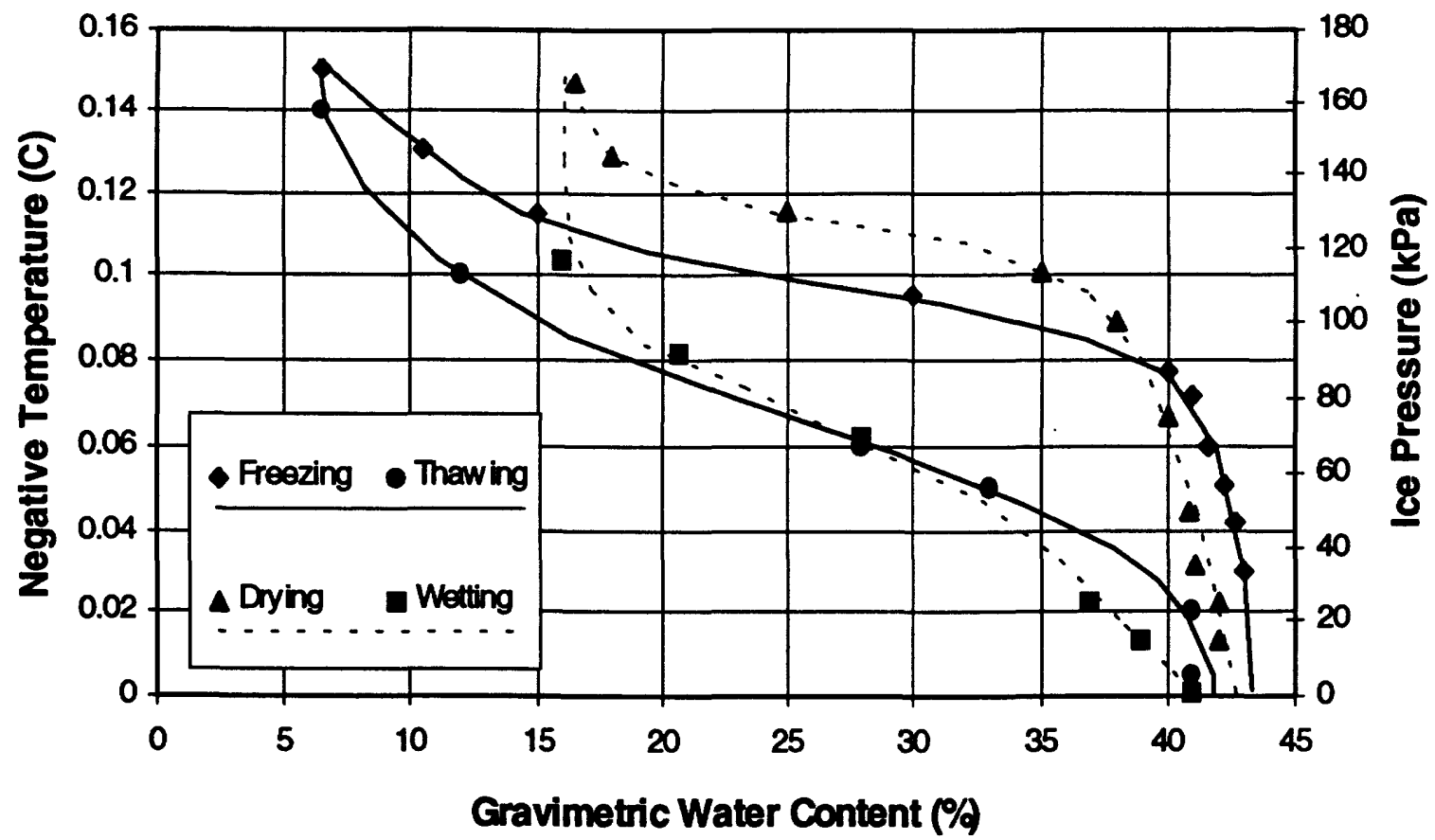

Figure 2.7 Experimental Relationships Between Soil Freezing Curves and Soil Water Characteristic Curves Including Hysteresis Effects (after Koopmans and Miller, 1966)

Application of the Kelvin equation to the ice-water and ice-air interface shows that a correction must be made to the relationship between soil water characteristic data and soil freezing data for soils wholly dominated by capillary forces. For the ice-water interface:

$$
\left(u_{i}-u_{w}\right)=\frac{\sigma_{i w}}{r_{i w}}
$$

where,

$\begin{array}{lll}\mathrm{u}_{\mathrm{i}} & = & \text { ice pressure }(\mathrm{kPa}), \\ \mathrm{u}_{\mathrm{w}} & =\quad \text { water pressure }(\mathrm{kPa}),\end{array}$ 
$\sigma_{\mathrm{iw}}=$ the surface tension between ice and water $(\mathrm{kN})$, and

$r_{\mathrm{iw}}=$ the radius of curvature of the interface $(\mathrm{m})$.

For the ice-air interface:

$$
\left(u_{i}-u_{a}\right)=\frac{2 \sigma_{\mathrm{gi}}}{r_{\mathrm{ai}}}
$$

where,

$\mathrm{u}_{\mathrm{a}}=$ the air pressure $(\mathrm{kPa})$,

$\sigma_{\mathrm{a}}=$ the surface tension between air and ice $(\mathrm{kN})$, and

$\mathrm{r}_{\mathrm{ai}}=$ the mean radius of curvature of the interface (m).

Koopmans and Miller (1966) experimentally determined the air-water, ice-water ratio (i.e., $\sigma_{\mathrm{aw}}: \sigma_{\mathrm{iw}}$ ) to be $2.2: 1$; and Miller (1973) calculated the ice-water, air-ice ratio (i.e., $\left.\sigma_{\mathrm{iw}}: \sigma_{\mathrm{ai}}\right)$ to be $1: 3.2$ by analysing the contact angle between interfaces.

Black and Tice (1989) correlated experimental soil water characteristic curve data to measured soil freezing data. They also presented unique power curve relationships which simultaneously represented both the soil freezing curve data and the soil water characteristic curve data for initially saturated soils composed of either coarse or fine material. An example of their experimental and computed results for a fine grained soil are shown in Figure 2.8 for the freezing / drying and thawing / wetting cases.

The general form of the Clapeyron equation used by Black and Tice (1989) is as follows:

$$
u_{w}-\frac{u_{i}}{\gamma_{i}}=\frac{L_{f}}{273.15} T^{*}
$$

where,

$\gamma_{i}=$ the specific gravity of ice (dec.),

$\mathrm{L}_{\mathrm{f}}=$ the volumetric latent heat of fusion $(\mathrm{kJ} / \mathrm{kg})$,

$\mathrm{T}^{*} \quad=\quad$ the freezing point depression of pore water $\left({ }^{\circ} \mathrm{C}\right)$. 
Equation 2.4 in its current form does not relate freezing point depression, $T^{*}$, to matric suction, $\left(\mathrm{u}_{\mathrm{a}}-\mathrm{u}_{\mathrm{w} .}\right)$. To make this connection, Black and Tice (1989) relate $\left(\mathrm{u}_{\mathrm{i}}-\mathrm{u}_{\mathrm{w}}\right)$ to $\left(u_{a}-u_{w}\right)$, using the ratios of surface tensions, $\sigma_{a w}: \sigma_{i w}$ discussed earlier. These correlations are presented below for both a coarse grained and fine grained soil.

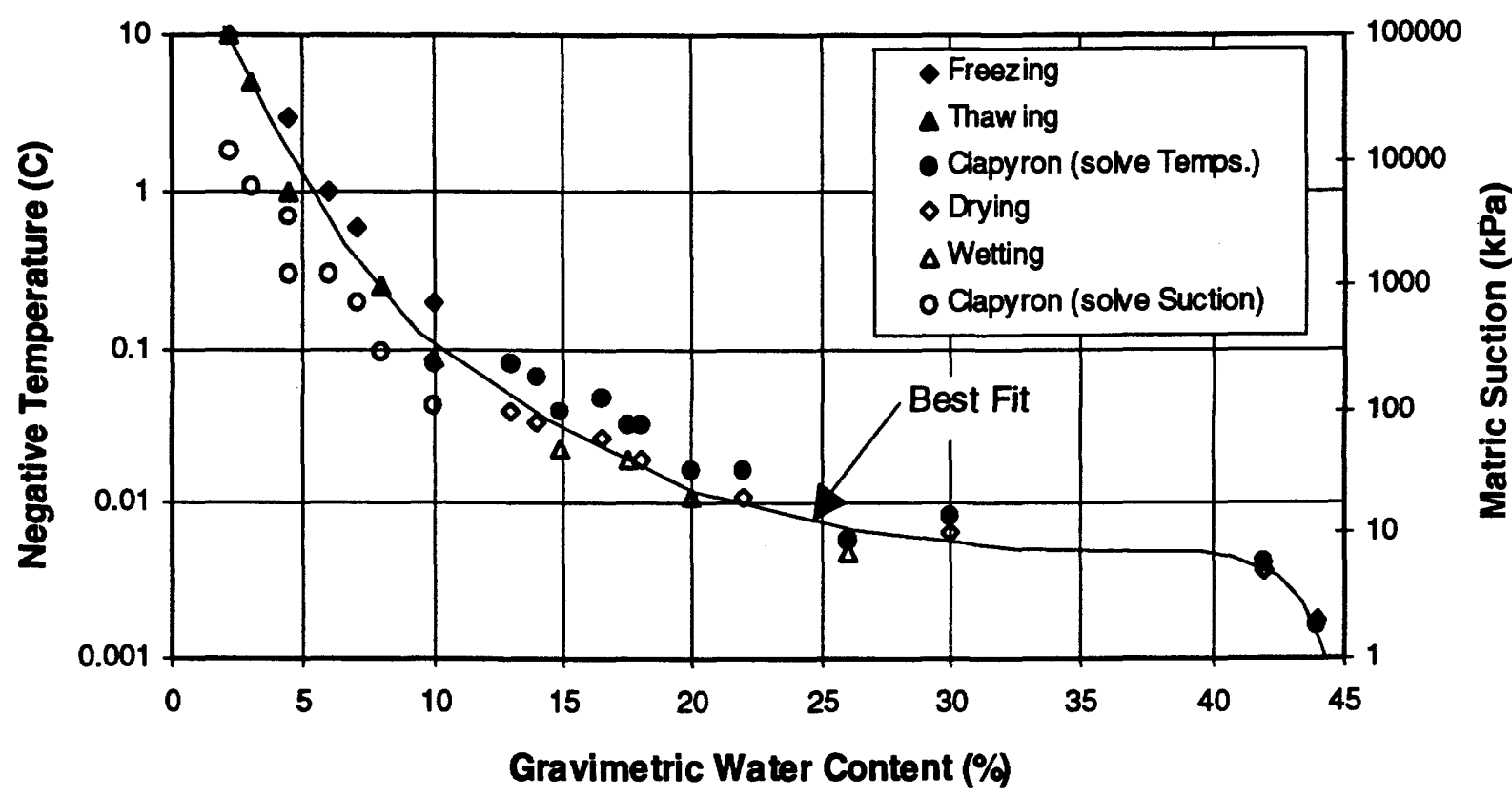

Figure 2.8 Combination of Experimental and Theoretical Soil Water Characteristic and Soil Freezing Curves for Windsor Sandy Loam (after Black and Tice, 1989)

When adsorptive forces $<<$ capillary forces (i.e., coarse grained):

$$
\begin{gathered}
\left(u_{a}-u_{w}\right)=\frac{\sigma_{a w}}{\sigma_{i w}}\left(u_{i}-u_{w}\right), \text { or } \\
\left(u_{a}-u_{w}\right)=2.2(1110) T^{*}
\end{gathered}
$$

When capillary forces << adsorptive forces (i.e., fine grained): 


$$
\begin{gathered}
\left(u_{a}-u_{w}\right)=\left(u_{i}-u_{w}\right), \text { or } \\
\left(u_{a}-u_{w}\right)=1110 T^{*}
\end{gathered}
$$

The constant value equal to $1110 \mathrm{kPa} /{ }^{\circ} \mathrm{C}$ combines the latent heat of fusion value, specific volume, and the conversion between the freezing temperature of water in Kelvins and degrees Celsius. The constant value of 2.2 is the ratio of surface tensions between air water, and ice - water in a coarse grained soil.

The above relationships may be useful for obtaining soil freezing curve data which can not be measured easily. For either soil type, the soil freezing data can be calculated using measured soil water characteristic curve data and the appropriate form of the Clapeyron equation as presented by Black and Tice (1989). Currently, no Clapeyron type formulation exists for soils which contain a mixture of capillary and adsorptive water forces.

The preceding discussions are significant to the development of the current soil freezing model because a Clapeyron type equation can couple the temperature and suction states in pore-water undergoing a phase change. Even though no theoretical Clapeyron equation exists for soils containing capillary and adsorptive water, it may be possible to obtain a relationship between suction and temperature using a measured soil freezing curve and a measured soil water characteristic curve. If both curves are known, then the freezing temperatures from the soil freezing curve should be inherently linked to the soil water suctions through the unfrozen water content which is common to both curves. This will be discussed again in Chapter 3.

Other empirical methods have been developed for predicting the unfrozen water content versus temperature or freezing point depression curve. Low et al. (1968) present a detailed thermodynamic technique and its instructions for use. Their method is quite complicated to apply and is most accurate in predicting large freezing point depressions. 
Tice et al. (1976) present a method which shows how to predict the unfrozen water content in soils from liquid limit data. Their method predicts the empirical constants, $\alpha$ and $\beta$ for use in the simple power curve relationship:

$$
\mathrm{w}_{\mathrm{u}}=\alpha\left(\mathrm{T}^{*}\right)^{\beta}
$$

where,

$\mathbf{w}_{\mathrm{u}}=$ the unfrozen gravimetric water content (dec.).

Their method gives very good correlation between measured and calculated values for liquid limits less than 100 and for relatively salt free soils. This type of power curve can also be used with values of $\alpha$ and $\beta$ which are determined experimentally. Table 2.1 shows some published unfrozen water content parameters for use in the above equation.

Anderson et al. (1973) recognize the applicability of the power curve shown in equation 2.9 , but they discuss the drawbacks of such a curve when used on clay-water systems. They suggest using a combination of two power curves. After testing eleven soil samples with specific surface areas ranging from 0.02 to $800 \mathrm{~m}^{2} / \mathrm{g}$ they were able to regress values of $\alpha$ and $\beta$ against specific surface area, $S$, as follows:

$$
\begin{aligned}
& \ln (-\beta)=-0.2640 \ln (S)+0.3711 \\
& \ln (\alpha)=0.5519 \ln (S)+0.2618
\end{aligned}
$$

Combining equations 2.10 and 2.11 with equation 2.9 results in an equation by which the gravimetric unfrozen water content can be determined for salt free soils at freezing temperatures when only the specific surface area is known. This equation is given below.

$$
\ln \left(\mathrm{W}_{\mathrm{u}}\right)=0.2618+0.5519 \ln (\mathrm{S})-1.449 \mathrm{~S}^{-0.264} \ln \left(\mathrm{T}^{*}\right)
$$


The following list summarizes the significant points presented in this section of the literature review.

1. Water in soils freezes below $0^{\circ} \mathrm{C}$.

2. Unfrozen water exists in frozen soils primarily due to negative pore-water pressures.

3. The unfrozen water content versus sub-zero temperature relationship applies regardless of the water content present when the soil first starts to freeze.

4. The Clapeyron and Kelvin equations provide a relationship between suction and temperature for soils dominated either by capillary or adsorptive water forces.

5. A relationship between temperature and suction states may be determined for a soil regardless of soil type if both the soil freezing, and soil water characteristic curves are known.

6. The soil freezing curve should ideally be measured in a laboratory, but other empirical methods of computing the necessary data have been developed. 
Table 2.1 Unfrozen Water Content Parameters For Use In Equation 2.9

\begin{tabular}{|c|c|c|c|}
\hline Soil Type & $\begin{array}{c}\text { Specific } \\
\text { Surface } \\
\text { Area }\end{array}$ & $\alpha$ & $\beta$ \\
\hline Morin Clay & 60 & 0.096 & -0.406 \\
\hline Morin Clay & 60 & 0.131 & -0.505 \\
\hline Caen Silt & - & 0.095 & -0.227 \\
\hline Calgary Silt & - & 0.096 & -0.364 \\
\hline Manchester Silt & - & 0.058 & -0.425 \\
\hline Kaolin & - & 0.104 & -0.245 \\
\hline Allendale Clay & - & 0.157 & -0.187 \\
\hline Inuvik Clay & - & 0.145 & -0.254 \\
\hline Tomokomai Clay & 54 & 0.195 & -0.305 \\
\hline Suffield Clay & 140 & 0.139 & -0.315 \\
\hline Fairbanks Silt & 40 & 0.048 & -0.326 \\
\hline nllite & 50.6 & 0.332 & -0.273 \\
\hline Fairbanks Silt & - & 0.074 & -0.384 \\
\hline $\begin{array}{l}\text { Undisturbed Fairbanks } \\
\text { Silt }\end{array}$ & - & 0.058 & -0.439 \\
\hline Chena Silt & 6 & 0.014 & -1.460 \\
\hline Japanese Clay (45\%) & - & 0.128 & -0.402 \\
\hline West Lebanon Gravel & 15 & 0.021 & -0.408 \\
\hline Manchester Silt & 18 & 0.025 & -0.515 \\
\hline Kaolinite (kGa-1) & 23 & 0.058 & -0.864 \\
\hline Chena Silt & 40 & 0.032 & -0.531 \\
\hline Leda Clay & 58 & 0.108 & -0.649 \\
\hline Morin Clay & 60 & 0.095 & -0.479 \\
\hline O’Brien Clay & 61 & 0.104 & -0.484 \\
\hline Goodrich Clay & 68 & 0.0864 & -0.456 \\
\hline Tuto Clay & 78 & 0.128 & -0.603 \\
\hline Sweden 478 Clay & 113 & 0.271 & -0.472 \\
\hline Suffield Silty Clay & 148 & 0.111 & -0.254 \\
\hline Frederick Clay & 159 & 0.140 & -0.279 \\
\hline Elleworth Clay & 184 & 0.112 & -0.293 \\
\hline Regina Clay & 291 & 0.211 & -0.238 \\
\hline Niagara Silt & 37 & 0.066 & -0.410 \\
\hline Norway LE-1 Clay & 52 & 0.099 & -0.523 \\
\hline Kaolinite No. 7 & 72 & 0.198 & -0.689 \\
\hline Athena Silt Loam & 83 & 0.060 & -0.301 \\
\hline Sweden 201 Clay & 106 & 0.197 & -0.492 \\
\hline Hectorite & 419 & 0.384 & -0.369 \\
\hline Volcanic Ash & 474 & 0.031 & -0.097 \\
\hline
\end{tabular}




\subsection{Thermal and Hydraulic Properties of Frozen Soils}

Modelling of transient freeze / thaw processes in soil requires that the thermal and hydraulic properties of the soils be known for both the freezing and non-freezing zones. From a heat transfer perspective it is necessary to know the thermal conductivity, volumetric specific heat capacity, and latent heat of fusion of water for the soil. From a mass transfer perspective, it is necessary to know the coefficient of permeability, and vapour diffusion coefficient. The unfrozen water content versus temperature relationship has been discussed in the previous section.

In the unfrozen zone, changes in thermal conductivity, volumetric specific heat, coefficient of permeability and vapour diffusion coefficient are a function of changes in water content. The relationships between these soil properties and water content are not valid in the frozen zone because the presence of ice changes the solid and liquid matrix of the soil. Excluding the coefficient of water permeability in frozen soils, there are generally well accepted methods of determining the required soil properties for both the freezing and non-freezing cases. These are discussed below.

\subsubsection{Thermal Conductivity}

Thermal conductivity is the amount of heat passing a unit cross-sectional area of soil, per unit time, under a unit temperature gradient. In equation form, it can be represented by:

$$
\lambda=\frac{\mathrm{qL}}{\mathrm{A}\left(\mathrm{T}_{2}-\mathrm{T}_{1}\right)}
$$

where,

$\lambda=$ the thermal conductivity $(\mathrm{W} / \mathrm{mK})$,

$\mathrm{q}=$ the heat flux per unit time $\left(\mathrm{W} / \mathrm{m}^{2}\right)$,

$\mathrm{L}=$ the length of flow $(\mathrm{m})$

A $=$ the cross-sectional area $\left(\mathrm{m}^{2}\right)$, and 
$T_{1,2}=$ the temperatures at each end of length, $L(K)$.

The thermal conductivity of the soil system is a function of the thermal conductivites and quantities of each individual component in the soil, and of the combination of soil components (i.e., soil density and porosity).

Farouki (1981) discusses and compares various methods for calculating the thermal conductivity of frozen and unfrozen soils. He discusses the sensitivity of each method with respect to soil type (fine or coarse), degree of saturation, mineral composition, and phase state (i.e., frozen or unfrozen). Farouki concludes that the method provided by Johansen (1975) gives the best results for frozen or unfrozen, coarse or fine soils, at various degrees of saturation above 0.1 . He adds that the method proposed by de Vries (1952) is more accurate for unfrozen coarse soils when the degree of saturation is between 0.1 and 0.2 . Below a saturation of 0.1 , none of the methods give good predictions. The method proposed by Kersten (1949) gives good results for frozen fine soils at a saturation below 0.9 , but this method does not apply for any coarse grained soil (frozen or unfrozen) with either a high or low quartz content. In saturated soils, several methods appear to compare favorably, but Farouki (1981) is of the opinion that the method proposed by Johansen (1975) is easiest to use.

The method given by Johansen (1975) expresses the thermal conductivity of an unsaturated soil as a function of the thermal conductivity in the dry and saturated states at the same dry density. The expressions listed below enable the thermal conductivity to be calculated for various cases. The main equation used by Johansen (1975) is:

$$
\lambda=\left(\lambda_{\text {sat }}-\lambda_{\text {dry }}\right) \lambda_{e}+\lambda_{\text {dry }}
$$

where,

$\lambda_{\text {sat }}=$ the saturated thermal conductivity $\left(\mathrm{W} / \mathrm{m}^{\circ} \mathrm{C}\right)$,

$=\quad 0.75^{\mathrm{n}} \lambda_{s}^{(1-n)}$ for the unfrozen case,

$=\quad 2.2^{\mathrm{n}} \lambda_{\mathrm{s}}^{(1-\mathrm{n})} 0.269^{\mathrm{W}_{\mathrm{v}}}$ for the frozen case, 


$$
\begin{aligned}
& \mathbf{w}_{\mathbf{u}}=\text { the unfrozen volumetric water content (dec.), } \\
& \mathrm{n}=\text { the porosity of the soil, } \\
& \lambda_{s}=\text { the effective solids thermal conductivity }\left(\mathrm{W} / \mathrm{m}^{\circ} \mathrm{C}\right) \text {, } \\
& =\quad 7.7^{q} 2.0^{1-q} \text { if } \mathrm{q}>0.20 \text {, } \\
& =\quad 7.7^{q} 3.0^{1-q} \text { if } q<0.20 \text {, } \\
& \mathrm{q}=\text { the quartz content as a fraction of total solids content(dec.), } \\
& \lambda_{\text {dry }}=\text { the thermal conductivity of the soil matrix in the dry state }\left(\mathrm{W} / \mathrm{m}^{\circ} \mathrm{C}\right) \text {, } \\
& =\frac{0.137 \gamma_{d}+64.7}{2700-0.947 \gamma_{d}} \text { if the soil is in a natural state, } \\
& =\quad 0.39 \mathrm{n}^{-2.2} \text { if the soil is crushed, } \\
& \gamma_{d}=\text { the dry density of the soil }\left(\mathrm{kg} / \mathrm{m}^{3}\right) \text {, } \\
& \lambda_{c}=\text { the Kersten number (dec.), } \\
& =\quad 0.7 \log \mathrm{S}_{\mathrm{r}}+1.0 \text { for a coarse, unfrozen soil, } \\
& =\quad \log \mathrm{S}_{\mathrm{r}}+1.0 \text { for a fine, unfrozen soil, } \\
& =\quad S_{\mathrm{r}} \text { for any frozen soil, } \\
& S_{\mathbf{r}}=\text { the degree of saturation (dec.), and } \\
& =\left(\theta_{\mathrm{i}}+\theta_{\mathrm{u}}\right) / \mathrm{n} \text {. }
\end{aligned}
$$

In the equations listed in above, the thermal conductivity of ice is assumed constant at 2.2 $\mathrm{W} / \mathrm{m}^{\circ} \mathrm{C}$, and that of water is $0.57 \mathrm{~W} / \mathrm{m}^{\circ} \mathrm{C}$.

The de Vries (1952) method was used by Harlan (1973) and subsequently Guymon and Luthin (1974), Jame (1977), Taylor and Luthin (1978), Guymon et al. (1980), and Flerchinger (1987). The equation is of the form as follows:

$$
\lambda=\frac{\sum_{j=1}^{n} F_{j} \theta_{j} \lambda_{j}}{\sum_{j=1}^{n} F_{j} \theta_{j}}
$$


where,

$\theta_{\mathrm{j}}=$ the volumetric content of the $\mathrm{j}^{\text {th }}$ component $\left(\mathrm{m}^{3} / \mathrm{m}^{3}\right)$,

$F_{j}=$ the weighting factor of the $j^{\text {th }}$ component (dec.),

$=\frac{1}{3} \sum_{n=1}^{3}\left\{1+\left(\frac{\lambda_{j}}{\lambda_{a i r}}-1\right) g_{n}\right\}^{-1}$

$\mathrm{g}_{\mathrm{n}}=\mathrm{g}_{1}+\mathrm{g}_{2}+\mathrm{g}_{3}=1$, a depolarization factor depending

on the shape of the component (dec.), and

$\lambda_{\text {air }}=\lambda_{\mathrm{a}}+\lambda_{\mathrm{v}}$, the thermal conductivity of air and

vapour $\left(\mathrm{W} / \mathrm{m}^{\circ} \mathrm{C}\right)$.

In the Jame (1977) study, the thermal conductivity of the soil solid, $\lambda_{j}$, was taken as that of pure quartz (i.e., $8.54 \mathrm{~W} / \mathrm{m}^{\circ} \mathrm{C}$ ), and the thermal conductivity of air, $\lambda_{\mathrm{a}}$, was taken as $0.025 \mathrm{~W} / \mathrm{m}^{\circ} \mathrm{C}$. For volumetric water contents above 0.2 , the vapour phase thermal conductivity, $\lambda_{v}$, was $0.736 \mathrm{~W} / \mathrm{m}^{\circ} \mathrm{C}$ and for water contents below 0.2 , the vapour thermal conductivity varied linearly from $0.0736 \mathrm{~W} / \mathrm{m}^{\circ} \mathrm{C}$ to zero at oven dryness. Water has a thermal conductivity of $0.573 \mathrm{~W} / \mathrm{m}^{\circ} \mathrm{C}$ and ice has a thermal conductivity of $2.176 \mathrm{~W} / \mathrm{m}^{\circ} \mathrm{C}$. Values of $g_{n}$ for the soil solid particles were chosen as $0.125,0.125$, and 0.75 ; which corresponds to particles having a shape of an ellipsoid of revolution. The values of $g_{1}$ and $\mathrm{g}_{2}$ for the air were assumed to decrease linearly from 0.333 in water saturated soils to 0.105 at a volumetric water content of 0.2 . Below this water content they varied linearly from a value of 0.105 to 0.015 at oven dryness. Values of $g_{n}$ for ice were chosen to be the same as the soil solid particles. Prior to using de Vries (1952) method of calculating thermal conductivity, Jame (1977) compared experiment values with theoretical values for the non-freezing case. The results of this comparison are illustrated in Figure 2.9. 


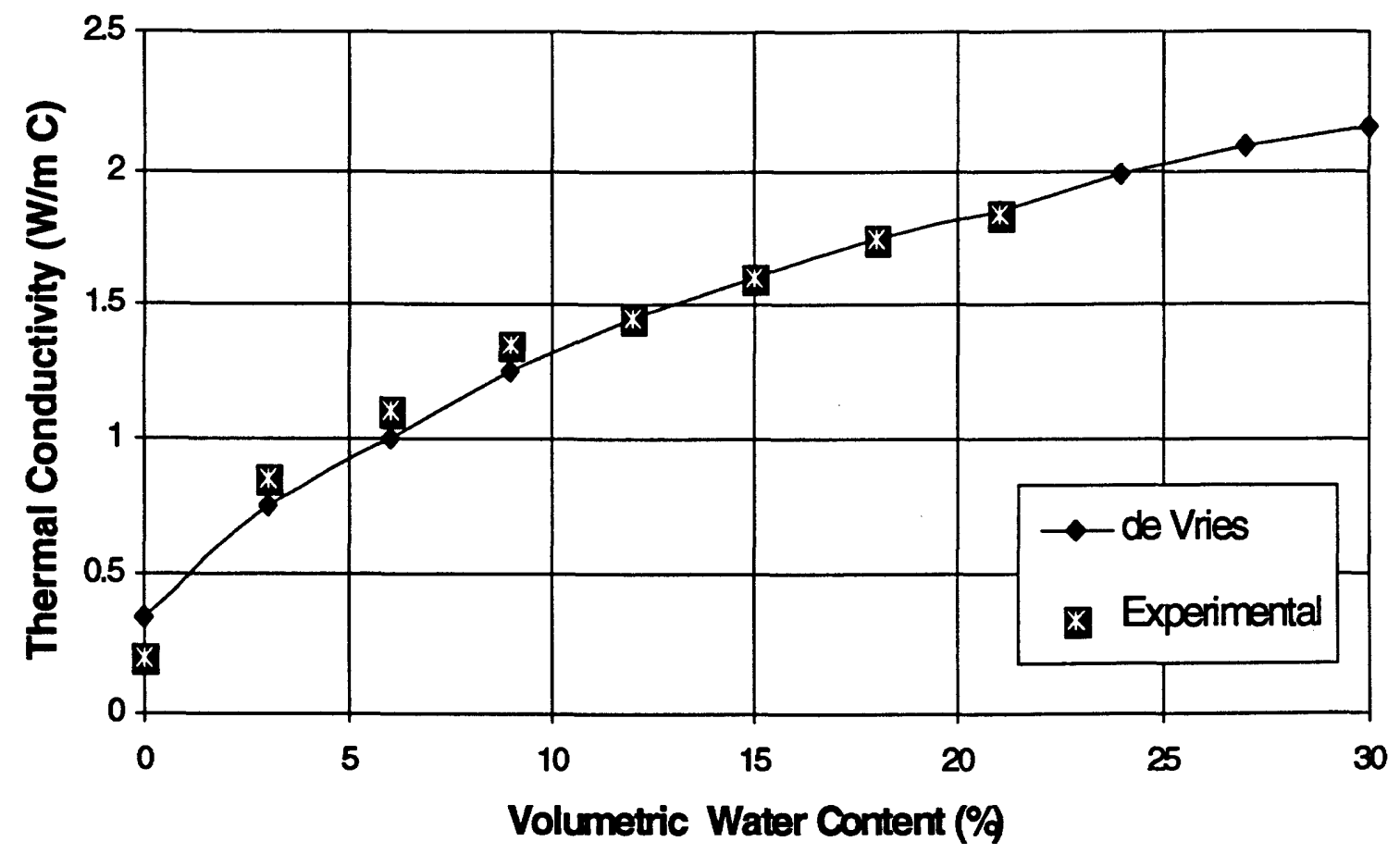

Figure 2.9 Comparison of Experimental and de Vries (1952) Method Thermal Conductivity for Non-Freezing Case (after Jame, 1977)

\subsubsection{Volumetric Specific Heat and Apparent Specific Heat}

If the temperature of a soil subjected to thermal gradients changes with time, then some of the heat is either stored or removed from the soil. The volumetric heat capacity of a soil is the heat energy required to raise the temperature of a unit volume of soil by $1{ }^{\circ} \mathrm{C}$. The volumetric heat capacity is the product of the mass specific heat, $c$ and the density, $\rho$ (Farouki, 1981). The volumetric specific heat of a soil-liquid-ice mixture can be estimated by the expression :

$$
c \rho=\sum_{j=1}^{n}(c \rho)_{j} \theta_{j}
$$

where,

$(c \rho)_{j}=$ the volumetric specific heat of the $\mathrm{j}^{\text {th }}$ component $\left(\mathrm{J} / \mathrm{m}^{3}{ }^{\circ} \mathrm{C}\right)$. 
If the dry density of the soil is known, equation 2.16 can be expressed as:

$$
c \rho=\gamma_{d}\left(c_{s}+4184 w_{u}+2100 w_{i}\right)
$$

where,

$\gamma_{\mathrm{d}}=$ the dry density of the soil solid $\left(\mathrm{kg} / \mathrm{m}^{3}\right)$,

$\mathrm{c}_{\mathrm{s}}=$ the mass specific heat of the soil $\left(\mathrm{J} / \mathrm{kg}{ }^{\circ} \mathrm{C}\right)$, and

$\mathrm{w}_{\mathrm{i}}=$ the gravimetric content of ice (dec.).

The mass specific heat of water and ice are $4184 \mathrm{~J} / \mathrm{kg}^{\circ} \mathrm{C}$ and $2100 \mathrm{~J} / \mathrm{kg}^{\circ} \mathrm{C}$ respectively. In the experiments performed by Jame (1972), a mass specific heat of silica flour solid was calorimetrically determined to be $837 \mathrm{~J} / \mathrm{kg}^{\circ} \mathrm{C}$. This is the same silica flour that is used for verifying the computer model developed in this current study.

In frozen soils the phase change is a gradual process, thus the term specific heat capacity is not strictly applicable (Anderson et al, 1973). In its place it is possible to use the term apparent volumetric specific heat capacity, $\overline{\rho c}$, originally defined by Williams (1964) as:

$$
\overline{\rho c}=\rho c+L_{f} \frac{\partial \theta_{u}}{\partial T}
$$

where,

$\overline{\rho C}=$ the apparent volumetric specific heat $\left(\mathrm{J} / \mathrm{m}^{3}{ }^{\circ} \mathrm{C}\right)$,

$\mathrm{L}_{\mathrm{f}}=$ the latent heat of fusion of water $(\mathrm{J} / \mathrm{kg})$, and

$\theta_{u}=$ the volumetric unfrozen water content, $\left(\mathrm{m}^{3} / \mathrm{m}^{3}\right)$.

The apparent volumetric specific heat incorporates the latent heat of fusion and the change in unfrozen water content with change in sub-zero temperature. 
The latent heat of fusion is known to vary with temperature and unfrozen water content. In their model, Guymon and Luthin (1974) use the following relationship to correct for the change in latent heat as follows:

$$
L_{f}=\rho_{u} L_{f}\left(\frac{\theta_{u}}{1-\theta_{s}}\right)
$$

where,

$\theta_{s}=$ the saturated volumetric water content $\left(\mathrm{m}^{3} / \mathrm{m}^{3}\right)$.

Anderson et al. (1973) report that corrections to the latent heat of fusion are only necessary at temperatures below approximately $-20^{\circ} \mathrm{C}$. In this current study the latent heat of fusion is assumed constant at $344 \mathrm{~kJ} / \mathrm{kg}$.

\subsubsection{Water Coefficient of Permeability}

It was discussed in section 2.1.2 that there is a large change in water permeability near and behind the freezing front in the frozen soil. Harlan (1973) makes the assumption that the coefficient of permeability versus water content (or suction) function is the same in the frozen and unfrozen zones at any given liquid water content. Harlan (1973), however, was unable to conclude that his hypothesis was valid, as his own numerical results showed that too much water and ice accumulated behind the freezing front and that the water content decreased too rapidly at the freezing front. Numerical modelling carried out by Jame (1977) also suggests that the assumption made by Harlan (1973) is invalid. Jame (1977) suggests that the presence of ice probably disrupts the established flow paths and hence reduces the flow rate.

To account for the reduced flow, Jame (1977) introduced an impedance factor of the form: 


$$
\mathrm{k}=\mathrm{k}_{\mathrm{h}} 10^{-\mathrm{E} \theta_{\mathrm{i}}}
$$

where,

$\mathrm{k}=$ the actual coefficient of permeability $(\mathrm{cm} / \mathrm{s})$,

$\mathbf{k}_{\mathrm{h}}=$ the unfrozen coefficient of permeability $(\mathrm{cm} / \mathrm{s})$, and

$\mathrm{E}=$ an empirical constant.

Jame (1977) calibrated his computer model by adjusting the empirical constant, E, in the above equation and by custom fitting a diffusivity versus water content function using data gathered from his initial tests. The permeability relationships then appeared to work well and give good results in other freezing simulations using the same material. Taylor and Luthin (1978), Hromadka (1987), Gosnik et al. (1988) and others have used the Jame (1977) approach and also obtained good computed results. Gosnik et al. (1988) report that typical values of ' $E$ ' are about 8 for fine sands and silts to 20 - 30 for coarser gravely soils. Black (1991) is very critical of the 'impedance factor' approach, stating that it is a "potent and wholly arbitrary correction function" for determining permeability. Results of the current study tend to support Black's opinion. This is discussed in Chapter 6.

Anderson et al. (1973) introduces a method for determining the coefficient of permeability in frozen soils. The Anderson et al. (1973) equation is of the form:

$$
\mathrm{k}=\frac{\mathrm{k}_{\mathrm{o}}}{-\mathrm{T}^{\alpha}}
$$

where,

$\mathbf{k}_{\mathrm{o}}=$ the coefficient of permeability at $-1^{\circ} \mathrm{C},(\mathrm{cm} / \mathrm{s})$, and

$\alpha=$ the slope of the K vs. $-\mathrm{T}$ on a $\log -\log$ plot. 
The $\alpha$ term is approximately equal to $-5 \beta$, where $\beta$ is the exponent parameter used in the power curve relationship given by Anderson et al. (1973) for unfrozen water content versus temperature functions (see Table 2.1 for some published values of the " $\beta$ " parameter). The coefficient of permeability at $-1^{\circ} \mathrm{C}$ is obtained from special tests described by Anderson et al. (1973).

Nixon (1992) compiled some coefficient of permeability values for frozen soils and plotted them on a log-log plot. These are shown in Figure 2.10.

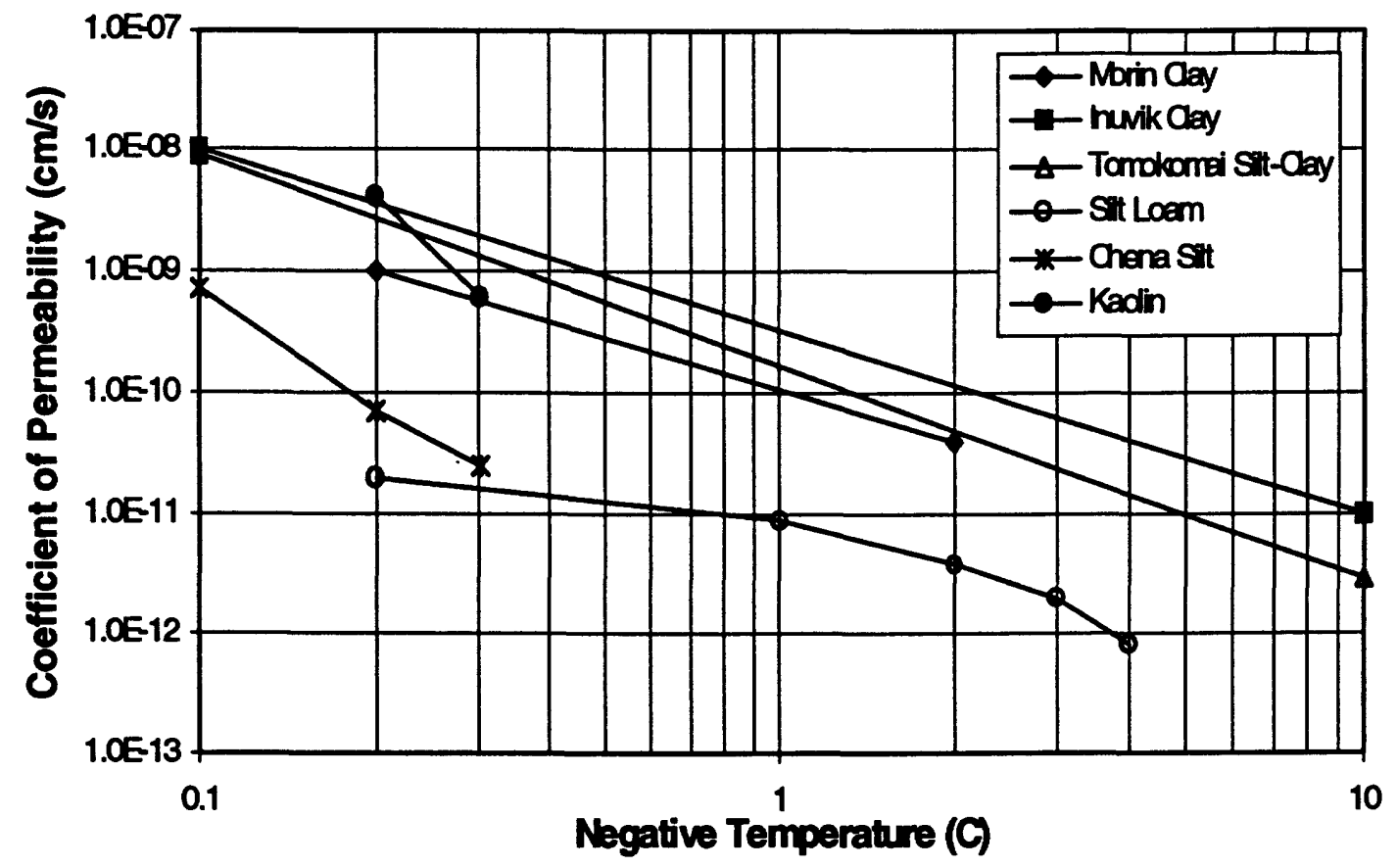

Figure 2.10 Hydraulic Conductivity of Frozen Soils (after Nixon, 1992)

Various other methods have been tried over the years. Tao (1994) used a power curve relationship first presented by Mualem (1976) that uses the normalized liquid saturation of the soil which is, itself, a function of volumetric ice content. Konrad and Morgenstern (1980) and Oliphant et al. (1982) present equations for determining the coefficient of permeability in the frozen zone which require parameters based on complex laboratory testing. Konrad and Morgenstern (1980) base their calculated coefficient of permeability 
on the soils segregation temperature (i.e., the temperature at which an ice lens starts to form). Nakano et al. (1982) base their calculation on the temperature gradient and the assumption that a form of the Clapeyron equation is valid for relating the pressure potential to temperature when both ice and liquid water phases are present.

Numerous studies have made no attempt to correct for the coefficient of permeability in frozen soils. Guymon and Luthin (1974) apply a form of the Gardner relationship for permeability in unsaturated soils to their freezing models. Flerchinger (1987) bases the permeability on suctions determined from a Brooks and Corey (1964) type calculation of matric potential. Flerchinger (1987) used his model to predict year round field moisture conditions with fairly good agreement between predicted and measured results.

To date, the literature shows that no single, acceptable method exists for determining the coefficient of permeability in a partially frozen, unsaturated soil. This is a major downfall to modelling freeze / thaw behaviour in soils. Until a suitable method of obtaining the frozen zone permeability is available, it is necessary to choose one of the approaches discussed above. One must either conduct extensive laboratory testing on freezing soils, or one must "calibrate" a model using measured data. This current study will compare results obtained with and without a permeability function correction for pore-ice blockage in the frozen zone.

\subsection{Numerical Models of Freezing / Thawing Soils}

Numerical models of soil freezing can be divided into two groups: those that assume the moisture content is static (i.e., considering only the thermal regime), and those that consider simultaneous coupled heat and mass transfer relationships. Extensive laboratory and field testing has shown that the former analysis is not sufficient. Thermal analysis does not account for any moisture redistribution and it does not give accurate temperature profiles in a partially frozen soil (Jame and Norum, 1980). 
The literature shows that there are three basic approaches to modelling heat and mass transfer in freezing soils. The capillary models (Penner, 1959; Williams, 1967) credit capillary suction at the ice / water interface for moving water toward a growing ice lens. Evidence has shown that capillary suction effects do not explain continued ice segregation under high overburden pressures (Smith, 1985).

The hydrodynamic models (Harlan, 1973; Guymon and Luthin, 1974; Taylor and Luthin, 1978; Jame, 1977; Jame and Norum, 1980; and others) use coupled heat and mass transfer relationships to model the complete soil regime above and below the frost line - with or without frost heave. Hydrodynamic models use some capillary theory and they require accurate predictions of coefficient of permeability in the both frozen and unfrozen zones. As discussed in the previous section, an arbitrary 'impedance factor' has been introduced to calibrate the hydraulic conductivity in these models.

The secondary frost heave approach (Miller, 1978) was developed with the objective of predicting ice lens formation and frost heave. It builds on the previously mentioned models and assumes that the criterion for the initiation of a new ice lens within the frozen fringe is the same as the criterion for initiation of an air-filled crack in unsaturated, unfrozen soils. This approach led to the rigid ice model (Miller, 1978) of coupled heat and mass transfer for a freezing front descending through air-free, solute-free incompressible soil. According to Black (1991), the rigid ice model has inherent computational difficulties which make it difficult for use in practical problems.

Other models, based on the hydrodynamic approach, have also been developed with the sole purpose of explaining and predicting frost heave phenomenon (Gilpin, 1980; Konrad and Morgenstern, 1980; Nixon, 1991). This latter group incorporate segregation pressures and temperatures, and special calculations for water permeability in the frozen zone which are functions of the uniquely defined segregation temperatures. 
The immediate application of the model proposed in Chapter 3 is to predict the thermal and moisture regime in a soil cover subjected to small overburden pressures and neglect any ice lensing. As a result, some form of a hydrodynamic model is most appropriate. The hydrodynamic model is discussed in more detail below.

\subsubsection{Hydrodynamic Soil Freezing Models}

The hydrodynamic model recognizes the coupled heat and mass transfers occurring simultaneously in freezing soils. The equations used in this approach are adapted from those used in unfrozen soils and are linked by the unfrozen water content versus temperature relationship and the Clapeyron equation.

The mass transfer equation given by Harlan (1973) is as follows:

$$
\frac{\partial}{\partial z}\left(k \frac{\partial \psi}{\partial z}\right)=\frac{\partial \theta_{u}}{\partial t}+\frac{\rho_{i}}{\rho_{u}} \frac{\partial \theta_{i}}{\partial t}
$$

where,

$\mathbf{k}=$ the hydraulic conductivity $(\mathrm{m} / \mathrm{s})$,

$\Psi \quad=\quad$ the soil water suction (m),

$\theta_{u}=$ the unfrozen volumetric water content $\left(\mathrm{m}^{3} / \mathrm{m}^{3}\right)$,

$\theta_{i}=$ the volumetric ice content $\left(\mathrm{m}^{3} / \mathrm{m}^{3}\right)$,

$\rho_{u}=$ the density of liquid water $\left(\mathrm{kg} / \mathrm{m}^{3}\right)$,

$\rho_{i}=$ the density of ice $\left(\mathrm{kg} / \mathrm{m}^{3}\right)$, and

$\mathrm{t}=$ time (s).

The heat transfer equation given by Harlan (1973) is as follows:

$$
\frac{\partial}{\partial z}\left(\lambda \frac{\partial T}{\partial z}\right)-\rho_{u} c_{u} V_{z} \frac{\partial T}{\partial z}=\rho c \frac{\partial T}{\partial t}-L_{f} \rho_{i} \frac{\partial \theta_{i}}{\partial t}
$$


where,

$$
\begin{aligned}
& \mathrm{z}=\quad \text { vertical position }(\mathrm{m}) \text {, } \\
& \lambda=\text { the thermal conductivity of the soil }\left(\mathrm{W} / \mathrm{m}^{\circ} \mathrm{C}\right) \text {, } \\
& \mathrm{T}=\text { temperature }\left({ }^{\circ} \mathrm{C}\right) \text {, } \\
& c_{u}=\quad \text { the mass specific heat of liquid water }\left(\mathrm{kJ} / \mathrm{kg}^{\circ} \mathrm{C}\right) \text {, } \\
& \mathrm{V}_{\mathrm{z}}=\text { the fluid flow velocity in the } \mathrm{z} \text {-direction }(\mathrm{m} / \mathrm{s}) \text {, } \\
& \text { pc = the volumetric heat capacity of the soil }\left(\mathrm{kJ} / \mathrm{kg}{ }^{\circ} \mathrm{C}\right) \text {, and } \\
& \mathrm{L}_{\mathrm{f}}=\text { the latent heat of fusion of water }(\mathrm{kJ} / \mathrm{kg}) \text {. }
\end{aligned}
$$

The second term on the left side of equation 2.23 is the convective heat transfer term which is often neglected (see comments in section 2.2). The two terms on the right side of the equation can be combined as:

$$
\frac{\partial}{\partial z}\left(\lambda \frac{\partial T}{\partial z}\right)-\rho_{u} c_{u} V_{z} \frac{\partial T}{\partial z}=\overline{\rho c} \frac{\partial T}{\partial t}
$$

where,

$$
\begin{aligned}
\overline{\rho c} & =\quad \text { the apparent volumetric specific heat }\left(\mathrm{kJ} / \mathrm{m}^{3}{ }^{0} \mathrm{C}\right), \\
& =\quad \rho c-L_{f} \rho_{i} \frac{\partial \theta_{i}}{\partial \mathrm{T}} \text { (Harlan, 1973; Jame, 1977), and } \\
& =\quad \rho c+L_{\mathrm{f}} \frac{\partial \theta_{\mathrm{u}}}{\partial \mathrm{T}} \text { (Anderson et al, 1973; Smith, 1985). }
\end{aligned}
$$

The apparent volumetric specific heat takes into account the latent heat of phase change during freezing or thawing. Therefore, the only modification to the heat transfer equation for freezing soils is the substitution of apparent volumetric specific heat for volumetric specific heat.

It should be noted that the apparent volumetric specific heat is a function of either the unfrozen volumetric water content or the volumetric ice content multiplied by ice density. 
This former version of the equation is convenient because the partial derivative term is simply the slope of the soil freezing curve. However, this form of the equation was derived for use in freezing analysis which neglects mass transfer.

Observation of equations 2.22 and 2.23 reveals that the heat and mass transfer equations are coupled by the change in ice content per change in temperature. The system of coupled equations can be solved either by finite difference or finite element methods assuming appropriate boundary conditions are applied. The solution strategies must be flexible enough to handle small time steps during the early stages of frost penetration. The grid spacings are also fairly small to increase the stability of the model. A detailed comparison of the advantages and disadvantages of each of various solution strategies and numerical procedures is beyond the scope of this literature review.

\subsection{Summary}

The physical processes taking place during soil freezing are not well understood and as a result the methods used to analyse freezing and thawing in soils are varied. Evidence clearly shows that both heat and mass transfer processes occur simultaneously and are coupled in both the frozen and unfrozen soil zones. Unfrozen water has been shown to exist in frozen soils at various freezing temperatures and it is this unfrozen water (along with some vapour flux in certain circumstances) which facilitates mass transfer behind the freezing front. Numerous analytical methods are available for predicting soil thermal properties in the frozen and unfrozen zones, but no generally accepted method exists to predict water permeability in the frozen zone. Various types of computer models have been proposed to predict heat and mass transfer in freezing soils and the complexity of these models increases when analysis of ice segregation is attempted. As the complexity of some of these models increase, the applicability of the models for practical use by engineers decreases. 
In this research program coupled heat and mass transfer equations will be derived for freezing and thawing unsaturated soils. The SoilCover (MEND 1993) model will be modified to verify proposed theory. Additional observations will be made about the necessity of using an ice impedance factor for calibrating the permeability function in the frozen zones. The revised numerical model will neglect ice lensing and convective heat transfer. Soil properties will be measured where possible or computed using the most appropriate and acceptable methods discussed previously in this chapter. The model will be verified using laboratory data collected by Jame (1977) and then applied to field data collected by O'kane (1995) from a waste rock cover at a mine site in the interior of British Columbia. 


\section{CHAPTER 3 THEORETICAL DEVELOPMENT}

\subsection{Introduction}

The primary objectives of this research program are to develop freezing theory for unsaturated soils and to devise a numerical solution technique for implementing theory into existing non-freezing soil heat and mass transfer models. This chapter describes the theoretical development and the numerical solution technique as it is implemented into the non-freezing SoilCover (MEND, 1993) model. Changes are made to the coupled heat and mass transfer equations, but, in order to model soil freezing, changes must also be made in the way soil thermal and hydraulic properties are determined. The background for the soil property changes was presented in the literature review chapter. The revised model verification program, the presentation of modelling results, and a discussion of the modelling results are presented in Chapter 4 , Chapter 5 and Chapter 6 respectively.

The SoilCover (MEND, 1993) model is a one dimensional finite element program which models transient water transport and heat flow in a soil profile. The model uses a physically based method for predicting the exchange of water and energy between the atmosphere and soil surface. Darcy's and Fick's laws are used to describe the flow of liquid water and vapour in the soil. Fourier's law for heat conduction and the latent heat of phase change between liquid and vapour phases describe the heat flow regime below the soil / atmosphere boundary. Evaporative fluxes from a saturated or unsaturated soil surface are predicted based on atmospheric conditions, vegetation cover and soil 
conditions. The modified Penman formulation (Wilson, 1990) is used to compute the actual rate of evaporation from the soil surface.

\subsection{Existing Heat and Mass Transfer Equations}

The heat and mass transfer equations used in SoilCover were derived by Wilson (1990) for the non-freezing case. The mass transfer equation is as follows:

$$
\frac{\partial h_{w}}{\partial t}=c_{w}^{1} \frac{\partial}{\partial z}\left(k_{w} \frac{\partial h_{w}}{\partial z}\right)+c_{w}^{2} \frac{\partial}{\partial z}\left(D_{v} \frac{\partial P_{v}}{\partial z}\right)
$$

where,

$\mathrm{h}_{\mathrm{w}} \quad=\quad$ total head $(\mathrm{m})$,

$\mathrm{t}=$ time $(\mathrm{s})$,

$c_{w}^{1}=\frac{1}{\rho_{u} g m_{2}^{w}} ;$ the modulus of volume change with respect to the liquid phase,

$\rho_{u} \quad=\quad$ density of water $\left(\mathrm{kg} / \mathrm{m}^{3}\right)$,

g = acceleration due to gravity $\left(\mathrm{m} / \mathrm{s}^{2}\right)$,

$\mathrm{z}=\operatorname{position}(\mathrm{m})$,

$k_{w}=$ the coefficient of permeability $(\mathrm{m} / \mathrm{s})$,

$c_{w}^{2}=\frac{P+P_{v}}{P\left(\rho_{u}\right)^{2} g m_{2}^{w}}$; the modulus of volume change with respect to the vapour phase,

$\mathrm{m}_{2}^{\mathrm{w}}=$ slope of the soil water characteristic curve $(1 / \mathrm{kPa})$,

$P=$ total gas pressure in the air phase $(\mathrm{kPa})$,

$\mathrm{P}_{\mathrm{v}}=$ partial pressure due to water vapour $(\mathrm{kPa})$,

$D_{v}=$ diffusion coefficient of water vapour through soil $(\mathrm{kg} \mathrm{m} / \mathrm{kN} \mathrm{s})$,

$$
=\quad \alpha \beta\left(D_{\text {vap }} \frac{W_{v}}{R T}\right),
$$

$\alpha \quad=\quad$ tortuosity factor of soil (dec.),

$=\quad \beta^{2 / 3}$ (dec.), 
$\beta=$ cross sectional area available for vapour flow (dec.),

$D_{\text {vap }}=$ molecular diffusivity of water vapour in air $\left(\mathrm{m}^{2} / \mathrm{s}\right)$,

$\mathrm{T}=$ temperature $(\mathrm{K})$,

$\mathrm{W}_{\mathrm{v}}=$ molecular weight of water $(0.18 \mathrm{~kg} / \mathrm{kmole})$, and

$R=$ universal gas constant $(8,314 \mathrm{~J} / \mathrm{mole} / \mathrm{K})$.

The heat transfer equation given by Wilson (1990) is:

$$
\mathrm{C}_{\mathrm{b}} \frac{\partial \mathrm{T}}{\partial \mathrm{t}}=\frac{\partial}{\partial \mathrm{z}}\left(\lambda \frac{\partial \mathrm{T}}{\partial \mathrm{z}}\right)-\mathrm{L}_{\mathrm{v}}\left(\frac{\mathrm{P}+\mathrm{P}_{\mathrm{v}}}{\mathrm{P}}\right) \frac{\partial}{\partial \mathrm{z}}\left(\mathrm{D}_{\mathrm{v}} \frac{\partial \mathrm{P}_{\mathrm{v}}}{\partial \mathrm{z}}\right)
$$

where,

$\mathrm{C}_{\mathrm{h}}=$ volumetric specific heat of the soil as a function of water content $\left(\mathrm{J} / \mathrm{m}^{3} /{ }^{\circ} \mathrm{C}\right)$,

$\lambda=$ thermal conductivity of the soil $\left(\mathrm{W} / \mathrm{m} /{ }^{\circ} \mathrm{C}\right)$, and

$\mathrm{L}_{\mathrm{v}}=$ latent heat of vapourization of water $(\mathrm{J} / \mathrm{kg})$.

Equations 3.1 and 3.2 are not in a form that can easily be applied to a finite element formulation since the coupling variable, $P_{v}$, is not one of the dependent variables (i.e., $T$, $h_{w}$ ). Joshi (1993) replaced the total head term in the mass transfer equation and the vapour pressure terms in both equations with an equivalent water pressure term, $\psi$. The resulting mass transfer equation given by Joshi (1993) for non-freezing soils is:

$$
\mathrm{m}_{2}^{\mathrm{w}} \frac{\partial \psi}{\partial \mathrm{t}}=\frac{\partial}{\partial \mathrm{z}}\left(\mathrm{k}_{\mathrm{w}} \frac{\partial}{\partial \mathrm{z}}\left(\frac{\psi}{\rho_{\mathrm{u}} \mathrm{g}}+\mathrm{z}\right)\right)+\frac{1}{\rho_{\mathrm{u}}} \frac{\partial}{\partial \mathrm{z}}\left(\mathrm{D}_{1} \frac{\partial \psi}{\partial \mathrm{z}}+\mathrm{D}_{2} \frac{\partial \mathrm{T}}{\partial \mathrm{z}}\right)
$$

where,
$D_{1}=\left(1 / \rho_{u}\right) D_{v} d_{1}\left(m^{3} s / k g\right)$,
$D_{2}=\left(1 / \rho_{u}\right) D_{v} d_{2}\left(m^{2} / \mathrm{C} s\right)$.
$d_{1}=\frac{P_{v} W}{\rho_{u} R T}($ dec.), 
$\mathrm{d}_{2}=\frac{\partial \mathrm{P}_{\mathrm{vs}}}{\partial \mathrm{T}} \mathrm{h}_{\mathrm{r}}-\frac{\mathrm{P}_{\mathrm{v}} \psi \mathrm{W}}{\rho_{\mathrm{u}} \mathrm{RT}^{2}}\left(\mathrm{~kg} / \mathrm{m} \mathrm{s}^{2 \circ} \mathrm{C}\right)$

$\Psi=$ soil matric suction $(\mathrm{kPa})$,

$=\quad-u_{w}$ when the pore-air pressure is assumed atmospheric, and

$\mathrm{u}_{\mathrm{w}}=$ the pore-water pressure $(\mathrm{kPa})$.

The heat transfer equation for non-freezing soils was modified by Joshi (1993) as follows:

$$
C_{h} \frac{\partial T}{\partial t}=\frac{\partial}{\partial z}\left(\lambda \frac{\partial T}{\partial z}\right)-L_{v} \frac{\partial}{\partial z}\left(D_{1} \frac{\partial \psi}{\partial z}+D_{2} \frac{\partial T}{\partial z}\right)
$$

\subsection{Derivation of the Modified Heat and Mass Transfer Equations for Freezing Soils}

Equations 3.3 and 3.4 represent the transient thermal and water pressure stress states in a soil for non-freezing conditions. In order to illustrate how these are modified for freezing conditions it is advantageous to begin with the water phase continuity equation for a partially frozen soil.

$$
\theta_{w}=\theta_{u}+\frac{\rho_{i}}{\rho_{u}} \theta_{i}
$$

where,

$\theta_{w}=$ the total volumetric moisture content in the soil $\left(\mathrm{m}^{3} / \mathrm{m}^{3}\right)$,

$\theta_{u}=$ the total volumetric liquid water content in the soil $\left(\mathrm{m}^{3} / \mathrm{m}^{3}\right)$,

$\theta_{i}=$ the total volumetric ice content in the soil $\left(\mathrm{m}^{3} / \mathrm{m}^{3}\right)$, and

$\rho_{i} \quad=\quad$ the density of ice $\left(\mathrm{kg} / \mathrm{m}^{3}\right)$.

The change in storage of total water content in a elemental volume of soil over time is equal to the magnitude of the flux terms on the right side of the mass transfer continuity 
relationship (i.e., Eq. 3.3). Substituting the liquid and vapour flux terms into the time derivative of the water phase continuity equation (i.e,. Eq. 3.5) results in:

$$
\frac{\partial \theta_{u}}{\partial t}+\frac{\rho_{i}}{\rho_{u}} \frac{\partial \theta_{i}}{\partial t}=\frac{\partial}{\partial z}\left(k_{w} \frac{\partial}{\partial z}\left(\frac{\psi}{\rho_{u} g}+z\right)\right)+\frac{1}{\rho_{u}} \frac{\partial}{\partial z}\left(D_{1} \frac{\partial \psi}{\partial z}+D_{2} \frac{\partial T}{\partial z}\right)
$$

If no freezing has taken place in the soil then the change in storage of unfrozen water is a function of the change in matric suction, and equation 3.6 reduces to equation 3.3. If freezing has taken place then the unfrozen water content is primarily a function of change in sub-zero temperature and its value is known from the soil freezing curve. For the freezing case, the change in unfrozen water content can be obtained using the slope of the soil freezing curve and the change in sub-zero temperature. Thus, equation 3.6 can be written as:

$$
\mathrm{m}_{2}^{\mathrm{i}} \frac{\partial \mathrm{T}}{\partial \mathrm{t}}+\frac{\rho_{\mathrm{i}}}{\rho_{\mathrm{u}}} \frac{\partial \theta_{\mathrm{i}}}{\partial \mathrm{t}}=\frac{\partial}{\partial \mathrm{z}}\left(\mathrm{k}_{\mathrm{w}} \frac{\partial}{\partial \mathrm{z}}\left(\frac{\psi}{\rho_{\mathrm{u}} \mathrm{g}}+\mathrm{z}\right)\right)+\frac{1}{\rho_{\mathrm{u}}} \frac{\partial}{\partial \mathrm{z}}\left(\mathrm{D}_{1} \frac{\partial \psi}{\partial \mathrm{z}}+\mathrm{D}_{2} \frac{\partial \mathrm{T}}{\partial \mathrm{z}}\right)
$$

where,

$\mathrm{m}_{2}^{\mathrm{i}}=$ the slope of the soil freezing curve $\left(1 /^{\circ} \mathrm{C}\right)$.

Equation 3.7 is the mass transfer equation for regions in a soil where freezing is occurring, or, where ice already exists.

The heat transfer equation is modified to include the latent heat of the phase change between liquid and solid phases by adding the appropriate term on the right side of equation 3.4 as follows: 


$$
C_{h} \frac{\partial T}{\partial t}=\frac{\partial}{\partial z}\left(\lambda \frac{\partial T}{\partial z}\right)-L_{v} \frac{\partial}{\partial z}\left(D_{1} \frac{\partial \psi}{\partial z}+D_{2} \frac{\partial T}{\partial z}\right)+L_{f} \rho_{u} \frac{\partial \theta_{i}}{\partial t}
$$

where,

$\mathrm{L}_{\mathrm{f}}=$ the latent heat of fusion of water $\left(334 \mathrm{~kJ} / \mathrm{kg}{ }^{\circ} \mathrm{C}\right)$.

Equations 3.7 and 3.8 are the heat flow and mass transfer continuity relationships for a freezing or partially frozen soil. The objective now, is to rearrange these equations such that they are solvable within the existing SoilCover program finite element formulation. Observation of the modified heat and mass transfer equations reveals two points. First, they are coupled by the partial vapour pressure variable and by the volumetric ice content variable. The assumption can be made that the primary coupling variable in a freezing soil is the volumetric ice content. This appears to be a reasonable assumption for the soil regions near the freezing front when one considers the relatively large volume of liquid water which changes phase to ice at this point compared with the volume of vapour which changes phase to liquid water. This may be a questionable assumption for regions well behind the freezing front where the primary mode of moisture transport is in the vapour phase. The second observation about the revised heat and mass equations is that there are three unknown variables (i.e., $T, \psi$, and $\theta_{i}$ ) and only two equations. Thus, the system of equations appears indeterminate.

Three steps need to be taken to render the system of equations determinate. First, the modified mass transfer equation (i.e., Eq. 3.7) is written such that the volumetric ice content term is isolated on the left side as follows:

$$
\frac{\rho_{i}}{\rho_{u}} \frac{\partial \theta_{i}}{\partial t}=\frac{\partial}{\partial z}\left(k_{w} \frac{\partial}{\partial z}\left(\frac{\psi}{\rho_{u} g}+z\right)\right)+\frac{1}{\rho_{u}} \frac{\partial}{\partial z}\left(D_{1} \frac{\partial \psi}{\partial z}+D_{2} \frac{\partial T}{\partial z}\right)-m_{2}^{i} \frac{\partial T}{\partial t}
$$


Second, the right hand side of equation 3.9 is substituted into the volumetric ice content term in the modified freezing heat transfer equation (i.e., Eq. 3.8) as follows:

$$
\begin{aligned}
\mathrm{C}_{\mathrm{h}} \frac{\partial \mathrm{T}}{\partial \mathrm{t}}= & \frac{\partial}{\partial \mathrm{z}}\left(\lambda \frac{\partial \mathrm{T}}{\partial \mathrm{z}}\right)-\mathrm{L}_{\mathrm{v}} \frac{\partial}{\partial \mathrm{z}}\left(\mathrm{D}_{1} \frac{\partial \psi}{\partial \mathrm{z}}+\mathrm{D}_{2} \frac{\partial \mathrm{T}}{\partial \mathrm{z}}\right)+\mathrm{L}_{\mathrm{f}} \frac{\rho_{\mathrm{u}}{ }^{2}}{\rho_{\mathrm{i}}} \frac{\partial}{\partial \mathrm{z}}\left(\mathrm{k}_{\mathrm{w}} \frac{\partial}{\partial \mathrm{z}}\left(\frac{\psi}{\rho_{\mathrm{u}} \mathrm{g}}+\mathrm{z}\right)\right) \\
& +\mathrm{L}_{\mathrm{f}} \frac{\rho_{\mathrm{u}}}{\rho_{\mathrm{i}}} \frac{\partial}{\partial \mathrm{z}}\left(\mathrm{D}_{1} \frac{\partial \psi}{\partial \mathrm{z}}+\mathrm{D}_{2} \frac{\partial \mathrm{T}}{\partial \mathrm{z}}\right)-\mathrm{L}_{\mathrm{f}} \frac{\rho_{\mathrm{u}}{ }^{2}}{\rho_{\mathrm{i}}} \mathrm{m}_{2}^{\mathrm{i}} \frac{\partial \mathrm{T}}{\partial \mathrm{t}} .
\end{aligned}
$$

Equation 3.10 is now the modified heat and mass transfer for the freezing zone in a soil. The above equation now contains two unknowns, $\Psi$ and $\mathrm{T}$; and it is still not solvable in this form as there are two unknown variables and only one equation.

Thermodynamic phase equilibrium in a freezing soil was considered in Chapter 2. At that point, the Clapeyron equation was introduced and shown to be useful (in certain circumstances) for relating suctions to temperatures in freezing soils. Various forms of the Clapeyron equation work adequately for soils that are wholly dominated by either adsorptive water forces or capillary water forces, but these relationships fail for soils containing a combination of capillary and adsorptive water forces. The general form of the Clapeyron equation for equilibrium between any two phases (Equation 2.1) is repeated again as follows:

$$
\frac{\mathrm{d} \psi}{\mathrm{dT}}=\frac{\Delta \mathrm{H}}{\mathrm{T} \Delta \mathrm{V}}
$$

where,

$\Delta \mathrm{H}=$ change in internal energy between phases $(\mathrm{kJ} / \mathrm{kg})$, and

$\Delta \mathrm{V}=$ change in specific volume between phases $\left(\mathrm{m}^{3} / \mathrm{kg}\right)$. 
Equation 3.11 clearly shows there is a unique relationship between suctions and temperatures in a material undergoing a phase change. The problem with using this type of relationship in freezing soils is that the equation is not clearly defined for all soil types (Black and Tice, 1989; Koopmans and Miller, 1966). Theoretically, it is possible to avoid this problem by combining data from the soil water characteristic curve and the soil freezing curve.

If the soil water characteristic curve and soil freezing curve are known, then for regions of soil where freezing is occurring, a unique relationship exits between suction and temperature. The slope of the soil freezing curve can divided by the slope of the soil water characteristic curve to give a value for the right side of equation 3.11 as follows:

$$
\frac{\partial \psi}{\partial T}=\frac{m_{2}^{i}}{m_{2}^{w}}=\frac{\partial \theta_{u}}{\partial T} \frac{\partial \psi}{\partial \theta_{u}}=G
$$

where,
$\mathrm{G}=$ the ratio between change in suction and change in temperature for a given unfrozen water content in a freezing soil $\left(\mathrm{kPa} /{ }^{\circ} \mathrm{C}\right)$.

Equation 3.12 can now be used to eliminate the suction variable in the combined heat and mass transfer equation (i.e., Eq. 3.10) so that only one equation with one unknown remains. Making this substitution and grouping like terms results in:

$$
\begin{aligned}
\left(\mathrm{C}_{\mathrm{h}}+\mathrm{L}_{\mathrm{f}} \frac{\rho_{\mathrm{u}}{ }^{2}}{\rho_{\mathrm{i}}} \mathrm{m}_{2}^{\mathrm{i}}\right) \frac{\partial \mathrm{T}}{\partial \mathrm{t}}=\frac{\partial}{\partial \mathrm{z}}\left(\lambda \frac{\partial \mathrm{T}}{\partial \mathrm{z}}\right)-\left(\mathrm{L}_{\mathrm{v}}-\mathrm{L}_{\mathrm{f}} \frac{\rho_{\mathrm{u}}}{\rho_{\mathrm{i}}}\right) \frac{\partial}{\partial \mathrm{z}}\left(\mathrm{D}_{1} \mathrm{G} \frac{\partial \mathrm{T}}{\partial \mathrm{z}}+\mathrm{D}_{2} \frac{\partial \mathrm{T}}{\partial \mathrm{z}}\right) \\
+\mathrm{L}_{\mathrm{f}} \frac{\rho_{\mathrm{u}}{ }^{2}}{\rho_{\mathrm{i}}} \frac{\partial}{\partial \mathrm{z}}\left(\mathrm{k}_{\mathrm{w}} \mathrm{G} \frac{\partial}{\partial \mathrm{z}}\left(\frac{\mathrm{T}}{\rho_{\mathrm{u}} \mathrm{g}}+\mathrm{z}\right)\right) .
\end{aligned}
$$


It is interesting to note that the term $\left(C_{h}+L_{f} \rho_{u}{ }^{2} / \rho_{i} m_{2}{ }^{i}\right)$ is the same as the "apparent specific heat" term used commonly in freeze / thaw analysis of soils. The first term on the right side of Eq. 3.13 is the conductive heat transfer term; the second term accounts for the net latent heat removed from the system due to phase changes from vapour to liquid and liquid to solid phases '; and the final term on the right side accounts for the liquid flowing into the system that changes phase and releases latent heat.

\subsection{Solution Strategy}

Equation 3.13 can be solved to give the soil temperature profile in the freezing or frozen soil zones. The suction profile in the freezing zone is obtained by looking up the suction which corresponds to the new unfrozen water contents given by the soil freezing curve for each newly solved temperature.. The modified numerical model uses the combined heat / mass transfer equation in the following way:

1. Using the previous time step suctions, the program computes the unfrozen water content from the soil water characteristic curve for every node in the finite element mesh. It then uses this unfrozen water content and the soil freezing curve to determine the freezing point depression temperature for every Gauss point. If a new Gauss point temperature is below the freezing point temperature, or if ice already exists at a Gauss point, then ice will, or may continue to form at the Gauss point during the next time step.

2. The program then computes the "apparent specific heat" and latent heat "ice flux" term constant values based on average thermal and hydraulic properties between the current and previous time steps.

\footnotetext{
${ }^{1}$ Note, this term is not a true sublimation term. It indirectly accounts for vapour - solid phase changes during freezing and solid - vapour phases changes during melting. It does not account for direct solid to vapour phase changes in a frozen soil (i.e., ice subliming to vapour without passing through a liquid phase).
} 
3. At each Gauss point where ice forms or already exists, the mass transfer equation in the frozen zone is 'turned off' and the modified freezing heat and mass transfer equation (i.e., Eq. 3.13) is 'turned on'. The program then solves for temperatures in the frozen zone, and for temperatures and suctions in the unfrozen zone.

4. At the end of each iteration, the suctions and ice contents are calculated using back substitution for each node in the frozen zone. The suctions are determined as mentioned above, and the ice contents are obtained by back substitution into the water phase continuity equation.

5. The iterations continue until the system converges based on temperature and suction at each node.

6. Ice contents at each node are stored in a global array to be used in soil thermal and hydraulic property calculations at the next time step. They are also used in checking the total water balance.

Figure 3.1 on the following page shows the flowchart algorithm for the modified numerical solution within the program's iteration subroutine where the element stiffness and mass matrices are computed. A complete listing of the revised computer code is given in the appendix. 


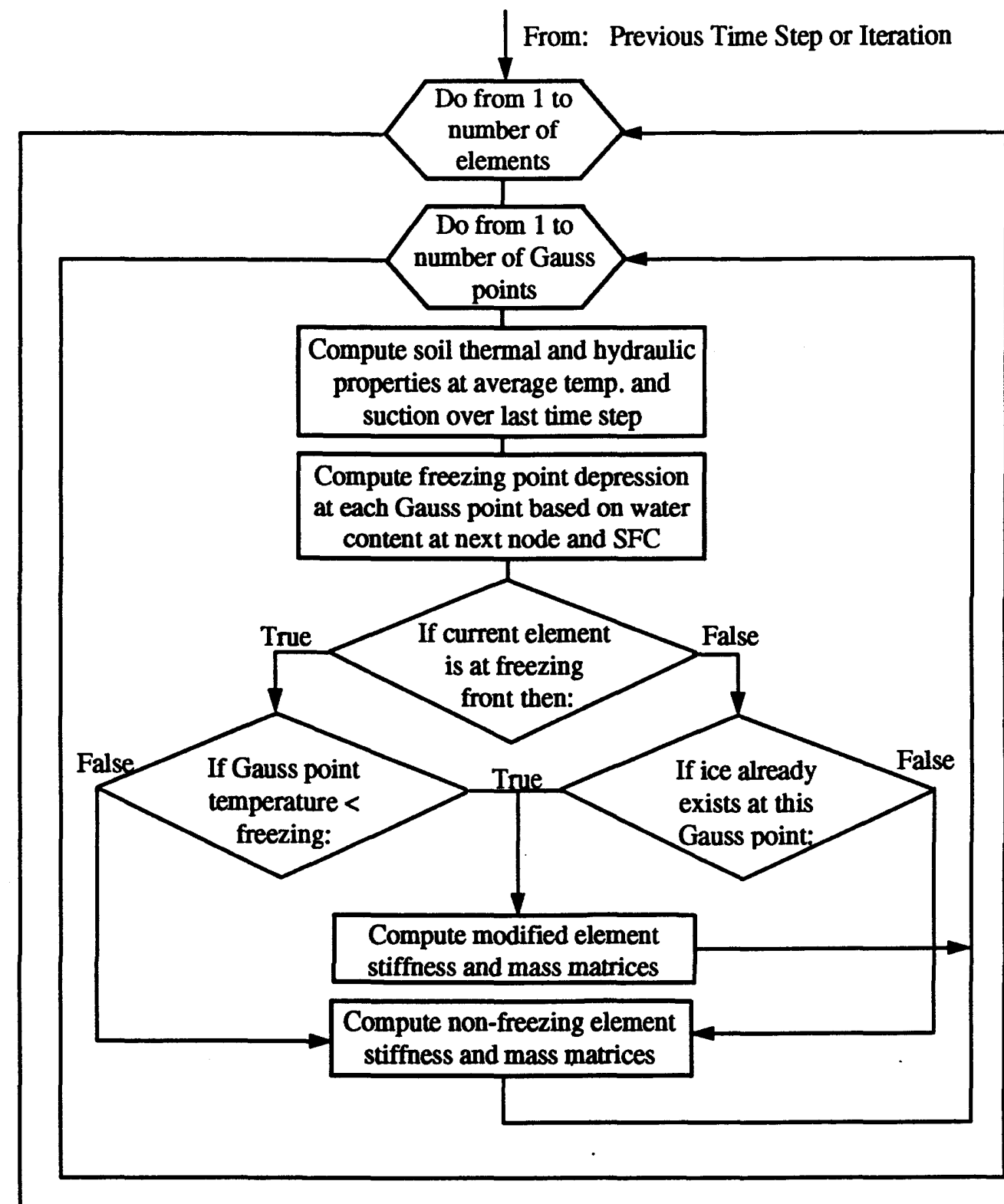

To: Assemble Global Matrices

Solve System of Equations

Check Convergence

New Iteration or Next Time Step

Figure 3.1 Flowchart Showing Criteria for Using Modified Soil Freezing Equation During Assembly of Element Stiffness and Mass Matrices 


\section{CHAPTER 4 REVISED MODEL VERIFICATION PROGRAM}

\subsection{Introduction}

The revisions to the SoilCover (MEND, 1993) program for freezing analysis were verified in two ways. First, it was necessary to verify that the theoretical formulations presented in Chapter 3 produced reasonable results when compared with carefully measured laboratory data. This verified that the dependent variables, suction and temperature, were being solved accurately in the revised finite element formulation. In addition, careful comparison with the laboratory data was necessary to determine if the computed values for ice content were acceptable. Finally, laboratory verification was necessary to ensure that the revisions to the soil property calculation functions were accounted for where necessary (i.e., that soil properties were modified to a account for ice content effects). Laboratory data verification did not take into account any thawing processes.

\subsection{Laboratory Data Modelling Program}

Jame $(1972,1977)$ carried out detailed investigations of freezing phenomenon in a fine grained silica flour material. In his initial work, Jame (1972) carried out experiments to determine the relationships between the unfrozen water content, sub-zero temperature, and freezing point depression for the silica flour. His later work (Jame, 1977; Jame and 
Norum, 1980) involved freezing of a horizontal column of silica flour while monitoring the temperature and total water content profiles with respect to time.

The material used by Jame $(1972,1977)$ was a \# 40 silica flour with $72 \%$ passing the \# 325 sieve. Jame (1977) prepared the silica flour at different initial moisture contents and packed it into lucite tubes, $30 \mathrm{~cm}$ in length and $10 \mathrm{~cm}$ in diameter. Jame estimated the dry density of the packed material to be $1.33 \mathrm{Mg} / \mathrm{m}^{3}$. Hollow brass circulation plates were placed at both ends of the column which were then sealed with wax so that no water could flow in or out of the system. Provision was made for air movement within the column and to ensure that the air pressure remained atmospheric. The apparatus was instrumented with twelve thermocouples at $2.5 \mathrm{~cm}$ intervals and insulated with Styrofoam and rock wool. Moisture contents were measured using the gamma ray attenuation method through $2 \mathrm{~mm}$ holes in lead blocks surrounding the sample.

Each experiment began by circulating cold fluids from temperature control baths through the brass circulation plates at each end of the column. The initial uniform temperatures of the samples ranged from $20^{\circ} \mathrm{C}$ to $5{ }^{\circ} \mathrm{C}$, depending on the test. Once the uniform initial temperature was reached, the temperature at one end of the column was maintained at the initial temperature while the other end of the column was cooled rapidly to the desired cold end temperature below $0{ }^{\circ} \mathrm{C}$. Moisture and temperature measurements were taken periodically over the $\mathbf{7 2}$ hour duration of each test. At the end of each test, gravimetric moisture content measurements were carried out to verify the moisture contents measured using the gamma ray method. More details of the experimental procedures and apparatus are given by Jame (1977).

The data in Table 4.1 summaries the initial conditions and boundary conditions for the three of the Jame (1977) tests. 
Table 4.1 Test Conditions for Jame (1980) Experiments

\begin{tabular}{ccccc} 
Test & $\begin{array}{c}\text { Initial Uniform } \\
\text { Temperature }\left({ }^{\circ} \mathrm{C}\right)\end{array}$ & $\begin{array}{c}\text { Initial Moisture } \\
\text { Content } \\
\text { \% by weight) }\end{array}$ & $\begin{array}{c}\text { Cold End } \\
\text { Temperature } \\
\left({ }^{\circ} \mathrm{C}\right)\end{array}$ & $\begin{array}{c}\text { Warm End } \\
\text { Temperature } \\
\left({ }^{\circ} \mathrm{C}\right)\end{array}$ \\
\hline 1 & 20 & 15.6 & -10 & 20 \\
2 & 5 & 15.0 & -5.9 & 4.25 \\
3 & 5 & 10.0 & -5.2 & 5.0
\end{tabular}

The results of the freezing tests conducted by Jame (1980) verify three hypotheses regarding the freezing of a fine grained, silty material. These are as follows:

1) There is a redistribution of water from the unfrozen zone to the frozen zone where it accumulates as ice.

2) There is a clearly defined freezing front as indicated by the change in water contents.

3) The ice content will build up behind the freezing front if the advancing frost front becomes somewhat stationary and water is free to flow.

Figures 4.1 through 4.3 show measured temperature and total water content profiles for the three freezing tests reported by Jame (1980). It should be noted that the total water content consists of both ice and liquid water in the frozen zone (i.e., left side of Figure) and only of liquid water in the unfrozen zone (i.e., right side of Figure). 

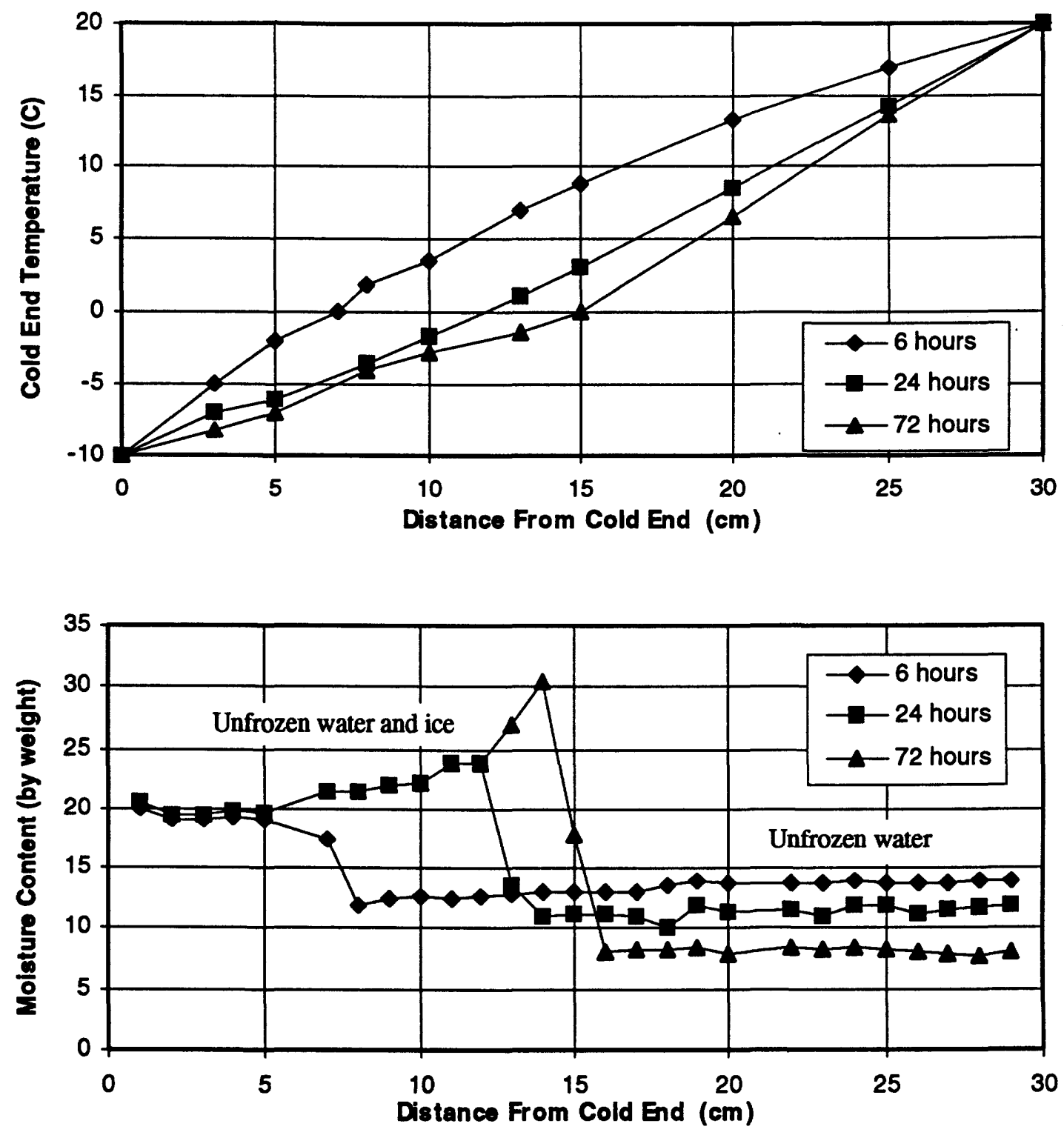

Figure 4.1 Experimental Results for Test 1 (after Jame, 1980) 

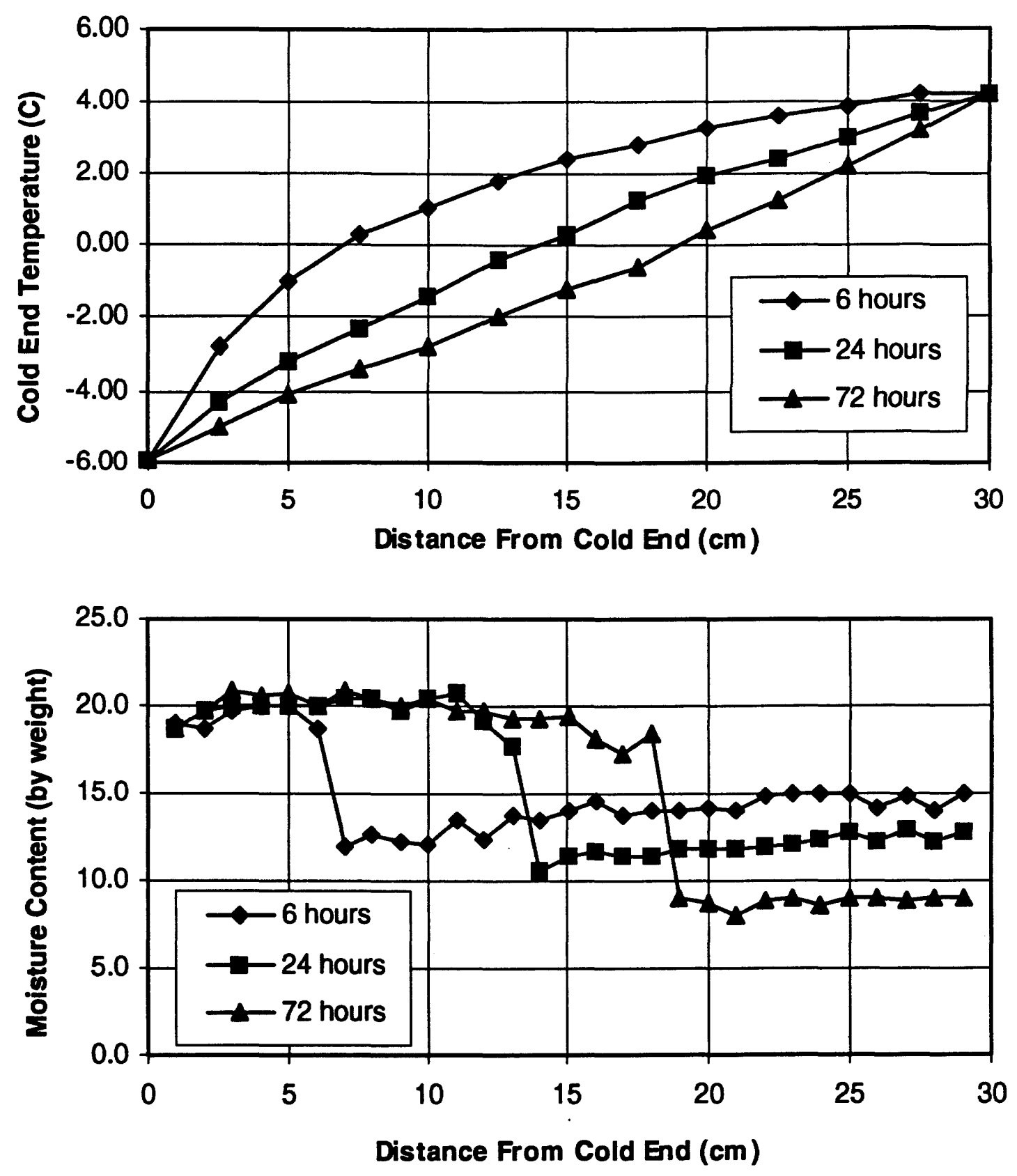

Figure 4.2 Experimental Results for Test 2 (after Jame, 1980) 

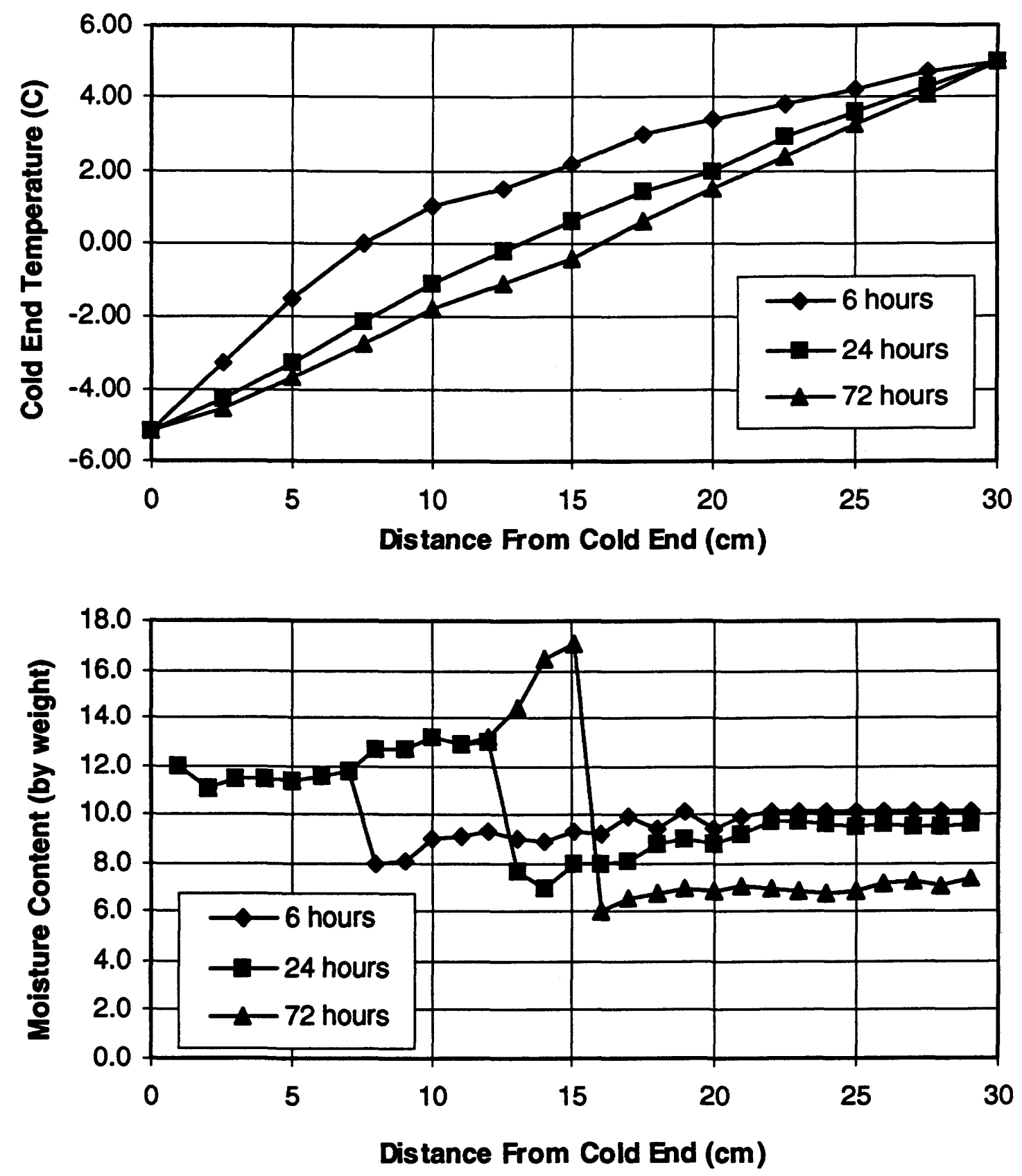

Figure 4.3 Experimental Results for Test 3 (after Jame, 1980) 


\subsection{Soil Properties Used in The Laboratory Data Modelling Program}

In order to model the experimental data reported by Jame (1980) it was necessary to determine the thermal and hydraulic material properties required as input in SoilCover. These properties included: the soil freezing curve (i.e., unfrozen water as a function of sub-zero temperature), the soil water characteristic curve, the saturated coefficient of permeability, the coefficient of permeability as a function of matric suction, the ice impedance factor for the frozen soil, the thermal conductivity as a function of total moisture content, and the volumetric specific heat as a function of total moisture content.

Jame (1977) used a silica flour that was no longer available for purchase for this study. However, a similar material for soil property measurements was obtained. Figure 4.4 below shows the approximate grain size curve of the Jame $(1972,1977)$ silica flour material and the measured grain size of the silica flour used in this study. The specific gravity of the material used in this study was measured to be 2.65. Jame (1977) did not report a specific gravity of the \# $\mathbf{4 0}$ silica flour used for that study.

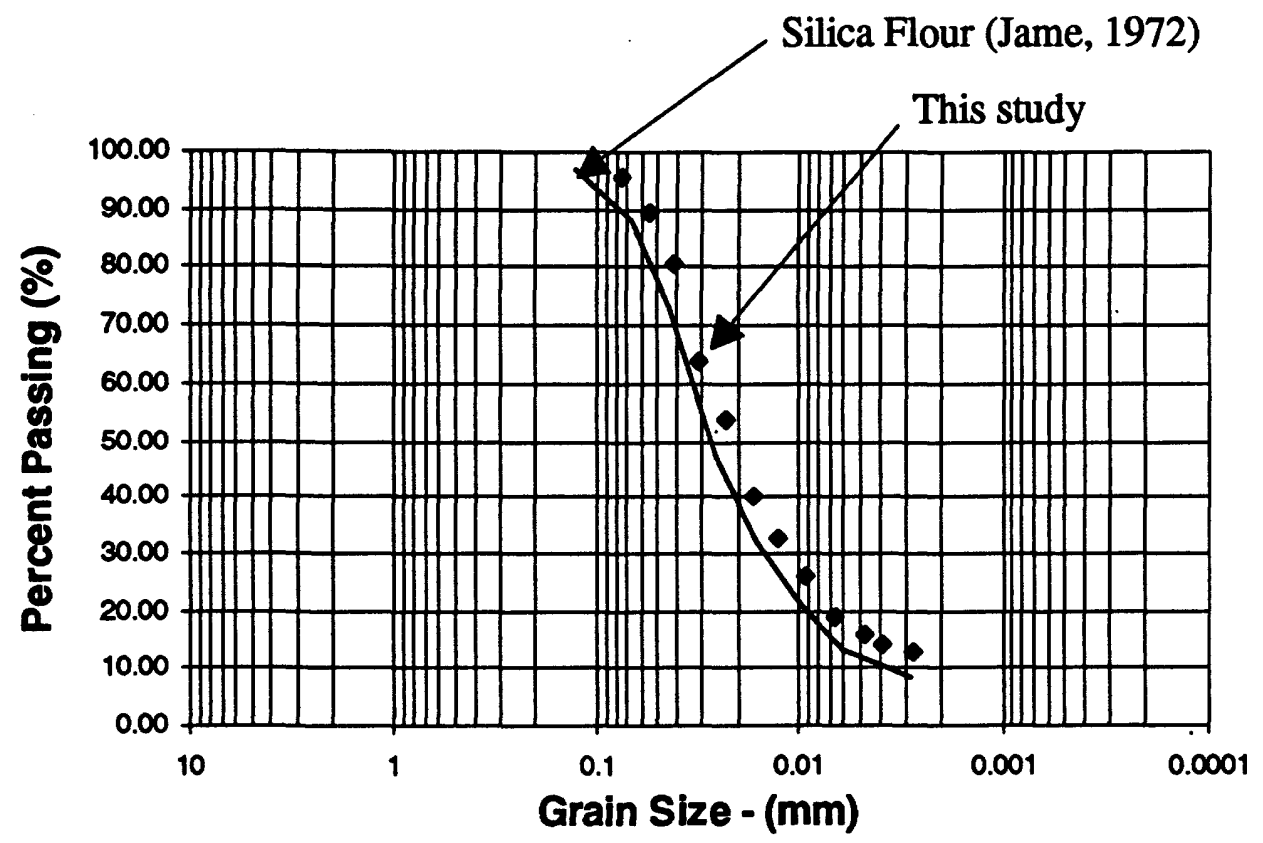

Figure 4.4 Grain Size Distribution for Silica Flour Soil Property Testing 
The results of the grain size distribution test show that the material used in this study was very similar to that used by Jame $(1972,1977)$. As a result, it was assumed that the freezing test experimental results obtained by Jame (1977) could be simulated using material properties obtained from soil property tests conducted on the silica flour used in this study.

The soil freezing curve for the silica flour used by Jame (1977) was discussed in the literature review chapter and is presented again in Figure 4.5. A semi - log plot of the soil freezing curve is shown in Figure 4.6. In this form it is similar in shape to a soil water characteristic curve.

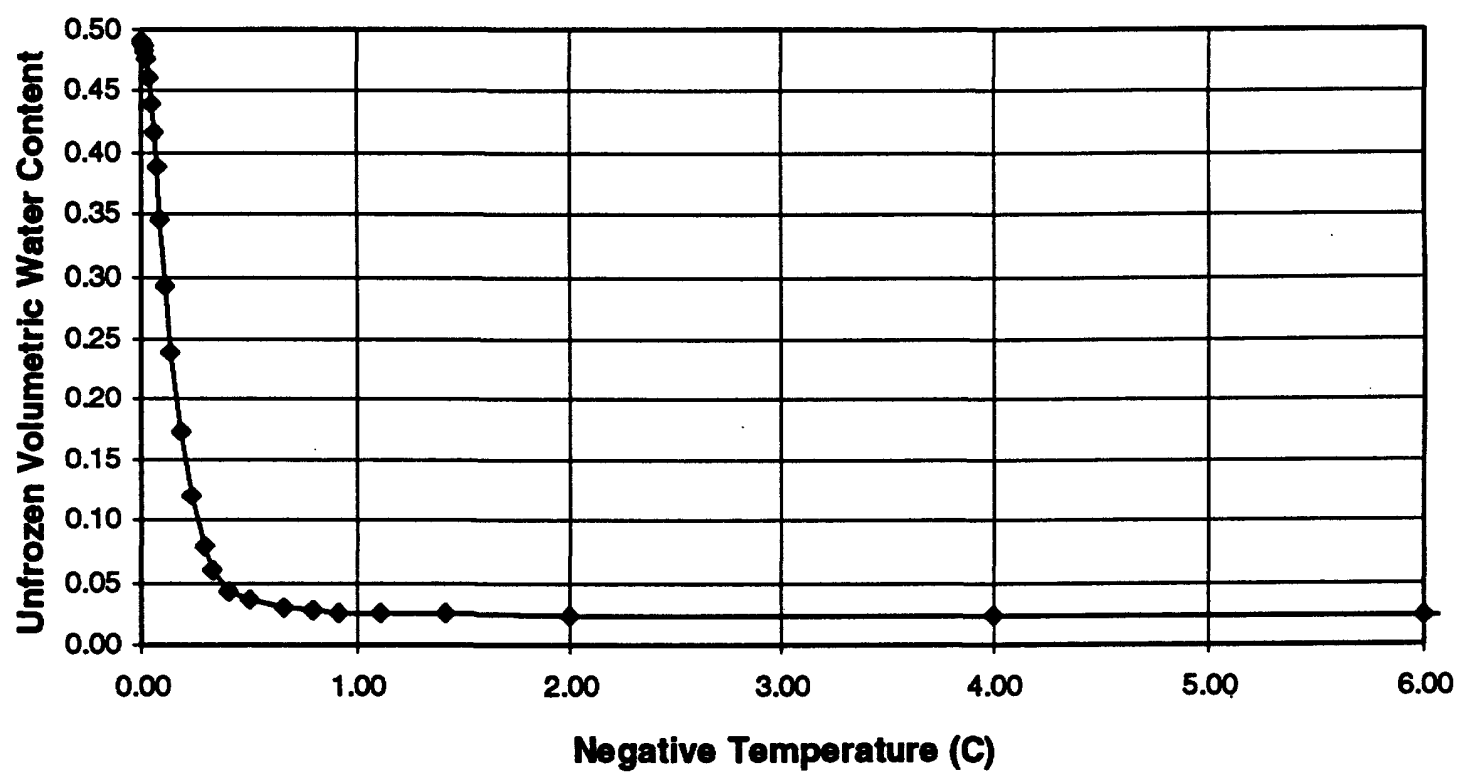

Figure 4.5 Soil Freezing Curve for Silica Flour (after Jame, 1972) 


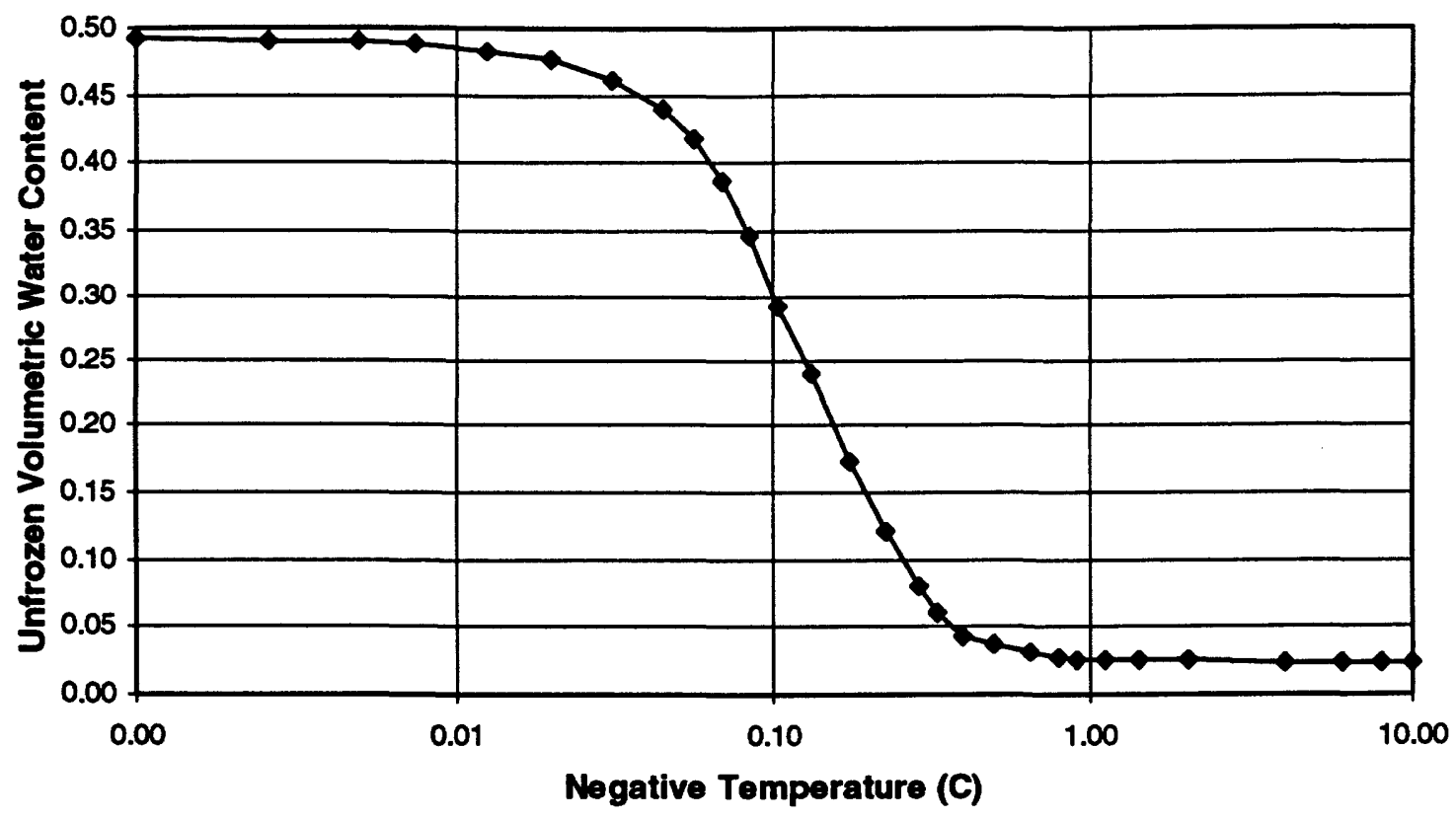

Figure 4.6 Semi - Log Plot of Soil Freezing Curve for Silica Flour

Data from the soil freezing curve given by Jame (1972) was used with a form of the Clapeyron equation given by Black and Tice (1989) to develop a theoretical soil water characteristic curve for the silica flour. This form of the Clapeyron equation relates matric suction to sub-zero temperatures in frozen soils dominated by capillary water forces. A comparison between the theoretical curve for Jame (1972) and the measured curve for this study is included in Figure 4.7.

The experimental soil water characteristic curve data was obtained using a modified Tempe cell with a 1 bar air entry disk. The 1 bar stone only permitted suctions up to 100 $\mathrm{kPa}$ to be applied to the sample. As a result, the suction values above $100 \mathrm{kPa}$ matric suction were estimated and plotted using the Fredlund and Xing (1994) equation for the soil water characteristic curve. The estimated portion of the curve was selected such that it approximated the theoretical values and approached a zero water content at 1 million $\mathrm{kPa}$ matric suction. A sensitivity comparison was performed to determine the effects of 
changing the residual matric suctions, and the slopes of the linear portion of the curve. This is discussed later in Chapters 5 and 6.

The term ' $G$ ' was introduced into the modified heat transfer equation for a freezing soil (i.e., Eq. 3.12). The term ' $G$ ' is the ratio of change in matric suction and change in subzero temperature in a freezing soil and it can be computed by dividing the slope of the soil freezing curve by the slope of the soil water characteristic curve for any given unfrozen volumetric water content. As such, ' $G$ ' may be considered unique for any soil type. Figure 4.8 shows a linearized form of the ' $G$ ' term as a function of volumetric water content for the silica flour used in this study.

Fredlund et al. (1994) present an equation which predicts the permeability function for unsaturated soils using the soil water characteristic curve. The function is an integrated form of the suction versus water content relationship and can relate permeability to suctions or water contents from zero water content to saturation (or $0 \mathrm{kPa}$ to 1 million $\mathrm{kPa}$ matric suction). Fredlund et al. (1994) verified the equation by fitting experimental data from various sources in the literature with accurate results. The equation was used in this study to predict a relative coefficient of permeability function based on the soil water characteristic curve. The relative coefficient of permeability versus matric suction relationship is shown in Figure 4.9.

The coefficient of permeability for the unsaturated soil was determined by multiplying the relative coefficient of permeability by the saturated coefficient of permeability, Ksat. A falling head permeameter was used to determine the saturated coefficient of permeability of the silica flour used in this study. The values of Ksat for the silica flour were found to range from $2.5 \times 10^{-4} \mathrm{~cm} / \mathrm{s}$ at a porosity of 0.52 , to $3.0 \times 10^{-5} \mathrm{~cm} / \mathrm{s}$ at a porosity of 0.48 . 


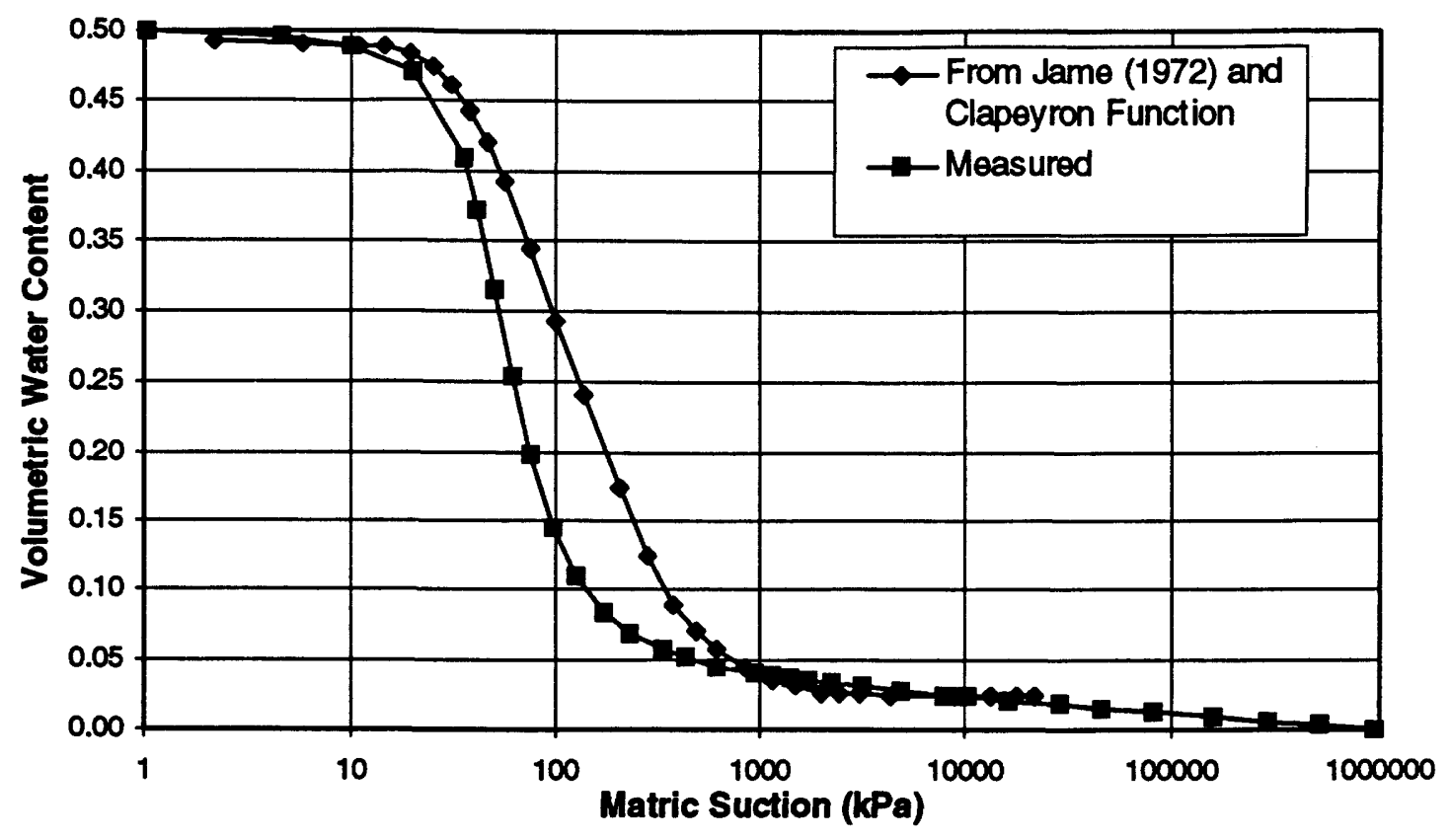

Figure 4.7 Theoretical and Experimental Soil Water Characteristic Curves (Measured Data Was Approximated at Suctions Above $100 \mathrm{kPa}$ )

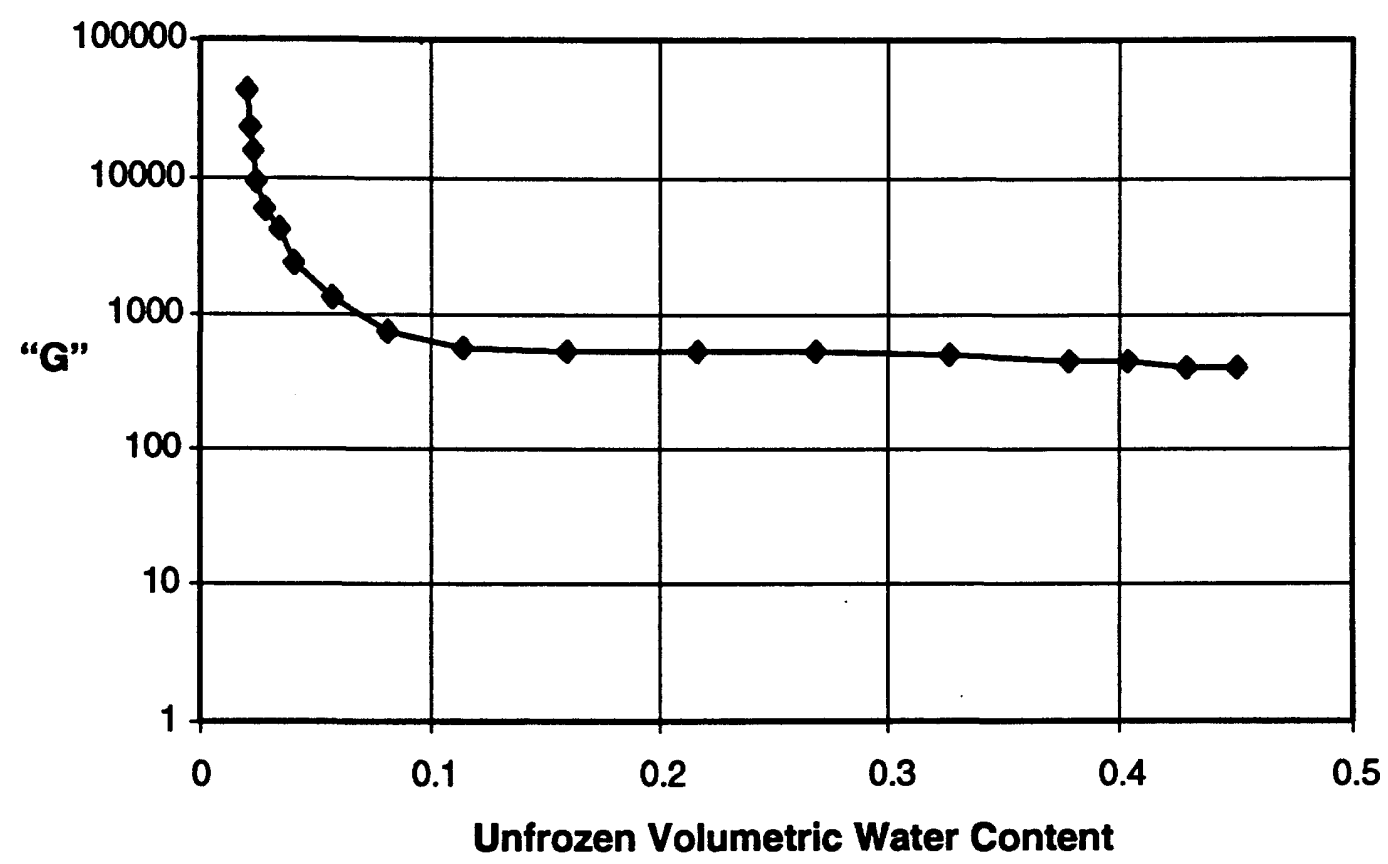

Figure 4.8 Ratio ' $G$ ' for Change in Matric Suction and Change in Sub-zero Temperature as a Function of Volumetric Water Content for Silica Flour 


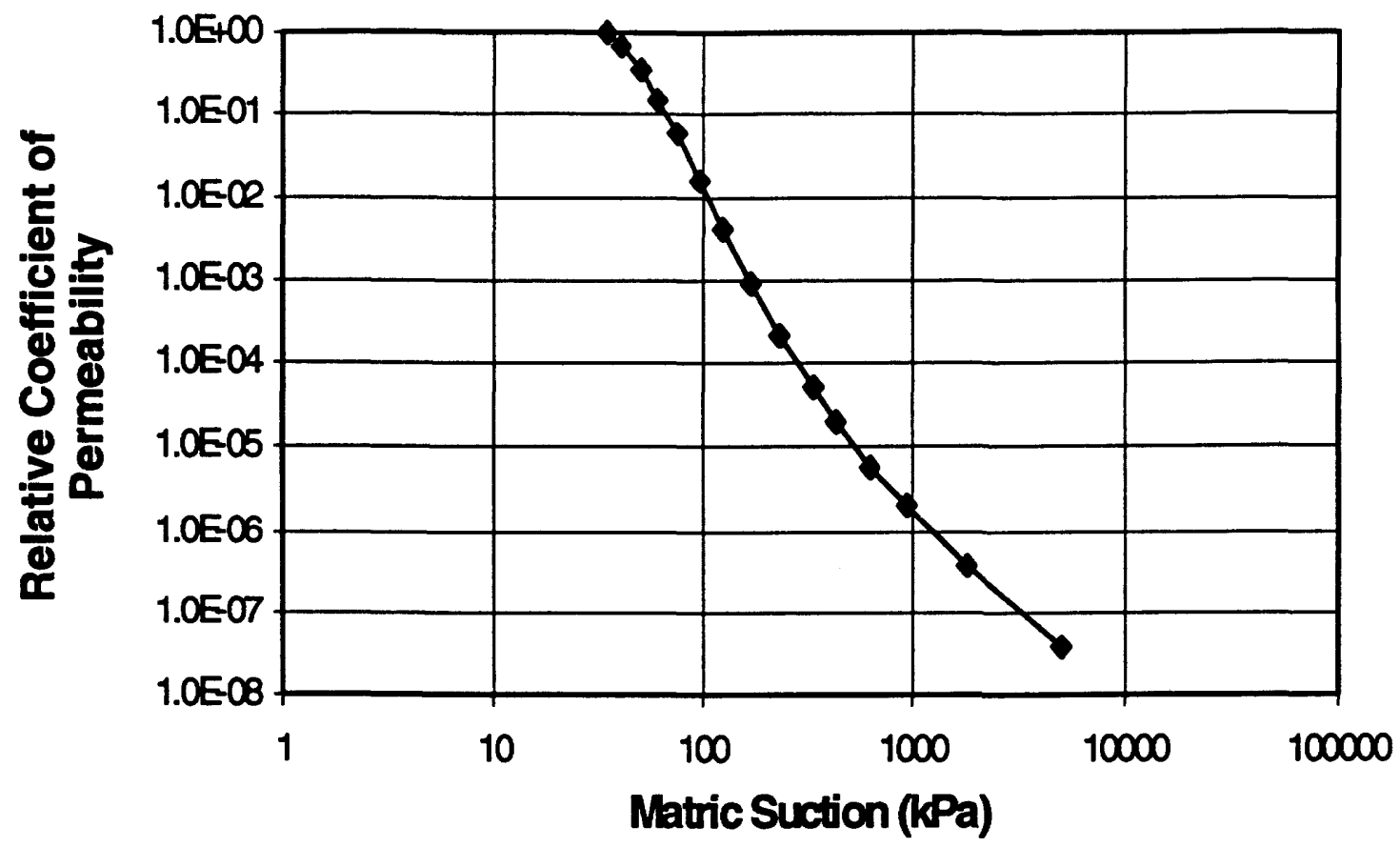

Figure 4.9 Relative Coefficient of Permeability for Silica Flour

Jame (1977) estimated the porosity of the silica flour material used to be 0.49 . The soil water characteristic curve used in this study was measured at a porosity of 0.51 . Because of this difference, three different saturated coefficients of permeability measured for the porosities in the range given above, were used during the computer simulations in this study. The sensitivity of the computed results with respect to small changes in the saturated coefficient of permeability for the silica flour is discussed in Chapters 5 and 6.

In numerous previous soil freezing heat flow and mass transfer models, researchers have adopted an impedance factor to account for the decreased water permeability in the frozen zone. They developed the impedance factor after initial computer simulations revealed too much ice was accumulating behind the frost front. Jame (1977) and Taylor and Luthin (1978) and others used an exponential impedance factor which was solely a function of volumetric ice content. In both these investigations, the freezing experiments performed by Jame (1977) were modelled using an impedance factor calculation of the form as follows: 


$$
\mathbf{k}\left(\psi, \theta_{\mathrm{i}}\right)=\mathbf{k}(\psi) \times 10^{-\left(\mathrm{E} \theta_{\mathrm{i}}\right)}
$$

where,

$\mathbf{k}(\Psi)=$ the coefficient of permeability from the suction versus permeability data $(\mathrm{m} / \mathrm{s})$, and

$\mathrm{E}=\quad$ an empirical constant equal to 12.

Applying this impedance factor with $\mathrm{E}=12$, has the effect of exponentially reducing the coefficient of permeability by three orders of magnitude as the volumetric ice content increases from 0.0 to 0.25 . Figure 4.10 shows the exponential nature of the ice impedance factor for a range of empirical constants and material types as suggested by Gosnik et al. (1988).

There has been a great deal of criticism of the 'impedance factor' (Black and Hardenberg, 1991) as it is often considered a means of getting the model to fit the data. In this study various impedance factors were used for comparison purposes, ranging from $E=0$ to $E=$ 12. The results of this comparison are presented in Chapter 5 and discussed in Chapter 6.

The thermal conductivity versus water content relationship for an unfrozen sample of the silica flour is shown in Figure 4.11. This Figure also compares experimental results obtained by Jame (1977) with theoretical approximations obtained using the methods proposed by de Vries (1963) and Johansen (1975). The method given by Johansen (1975) is much easier than de Vries (1963) method and according to Farouki (1981) gives superior results for a wider range of soil types and water contents. As a result, the Johansen (1975) method for computing the thermal conductivity of a frozen soil was chosen in this study during computer simulations. The thermal conductivity in the unfrozen zone was obtained directly from the data of Figure 4.11. For details regarding the application of the Johansen (1975) method see Chapter 2. 


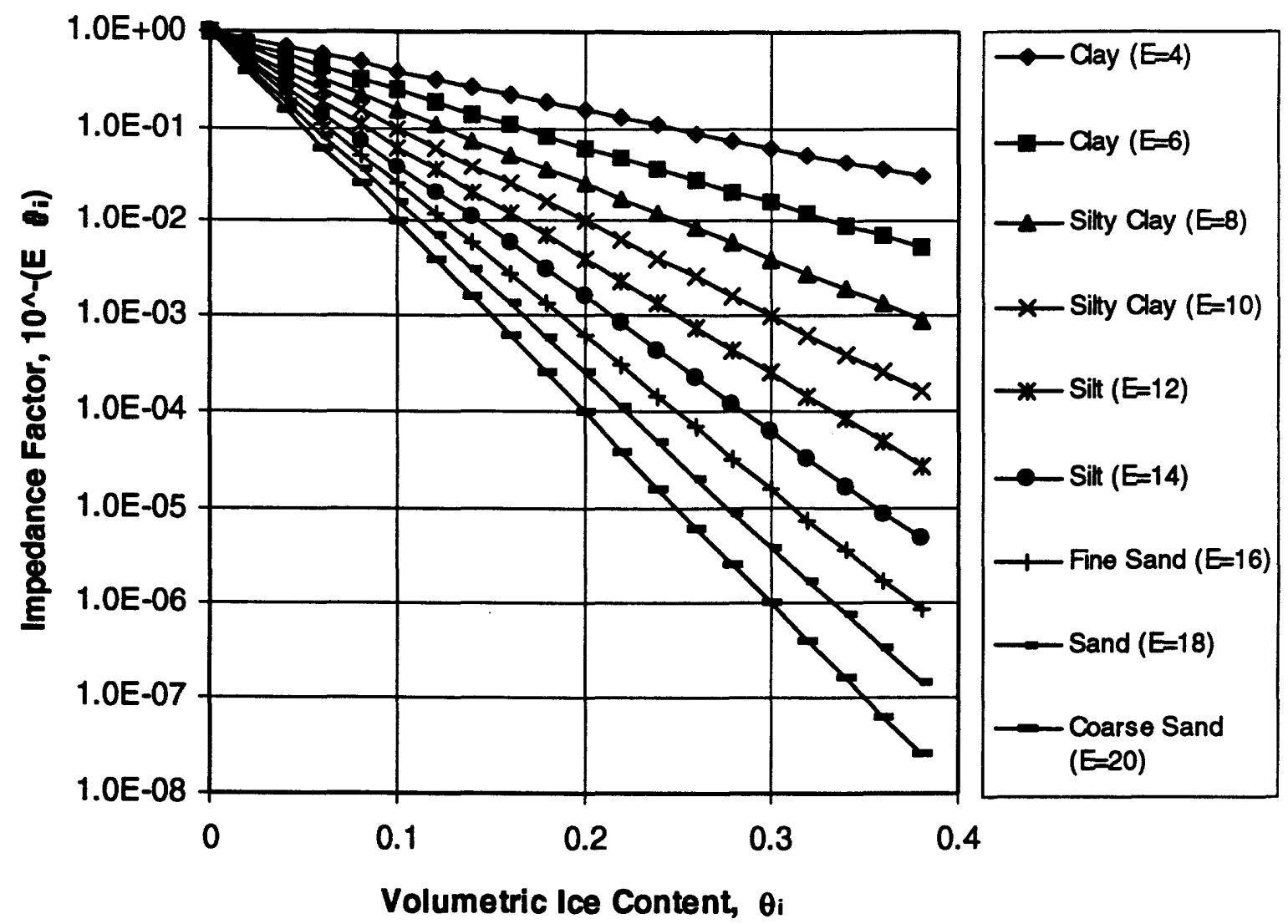

Figure 4.10 Coefficient of Permeability Ice Impedance Factors for Various Soil Types (after Gosnik et al. 1988)

Several parameters are required for the Johansen (1975) method thermal conductivity calculations. The thermal conductivity of the silica flour solid particle, $\lambda_{s}$, was assumed by Jame (1977) to be that of pure quartz at $8.54 \mathrm{~W} / \mathrm{m}{ }^{0} \mathrm{C}$. Johansen (1975) suggested a value for $\lambda_{s}$ of $7.7 \mathrm{~W} / \mathrm{m}^{0} \mathrm{C}$. In this study, a value of $8.12 \mathrm{~W} / \mathrm{m}^{0} \mathrm{C}$ seemed to give good agreement between measured and computed thermal conductivities as shown in Figure 4.11. The dry thermal conductivity of the mixture, $\lambda_{d}$, required in the Johansen (1975) method was measured by Jame (1977) to be approximately $0.25 \mathrm{~W} / \mathrm{m}^{0} \mathrm{C}$. 


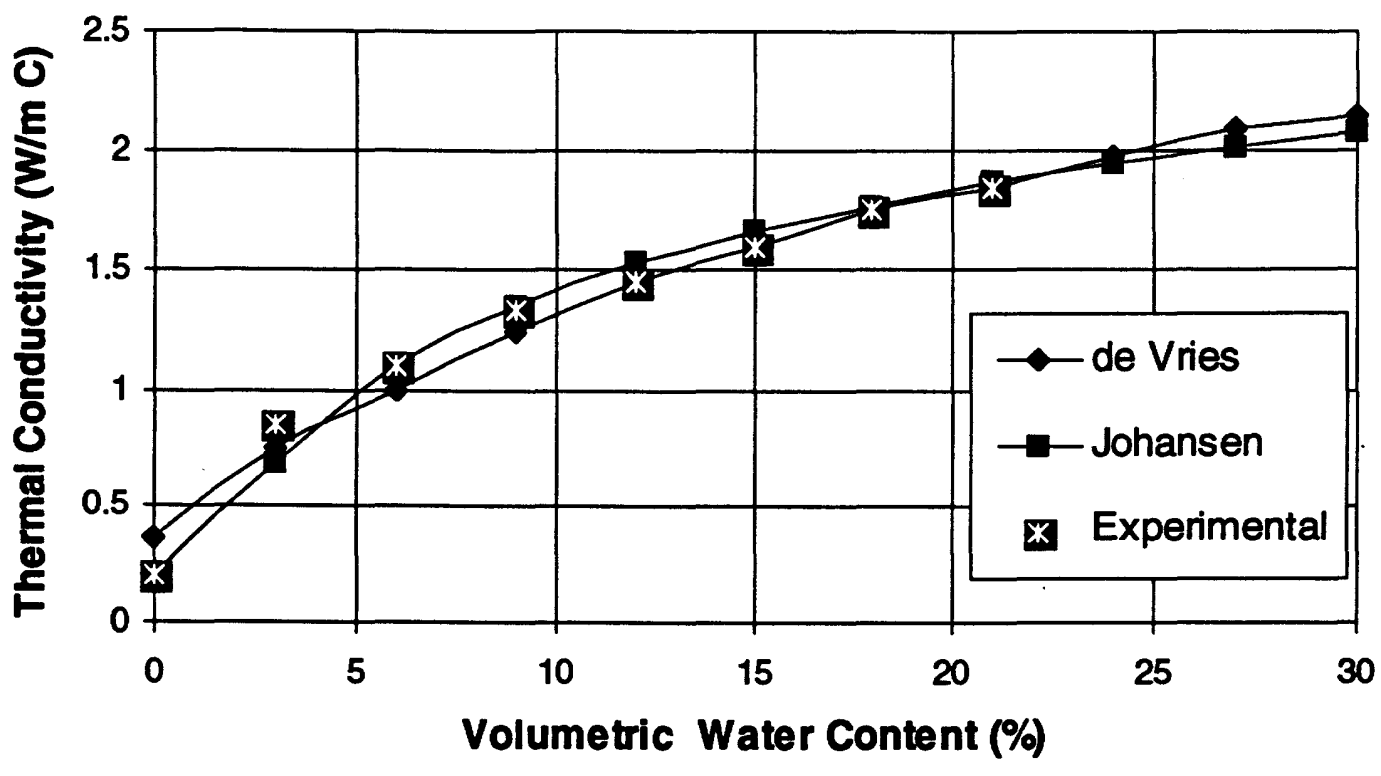

Figure 4.11 Thermal Conductivity for Unfrozen Silica Flour

Figure 4.12 shows the thermal conductivity of the frozen silica flour as a function of subzero temperature. The volumetric ice contents were obtained by subtracting the unfrozen water content ( as given by the soil freezing curve -i.e., Figure 4.4) from an arbitrarily chosen initial water content for a range of temperatures below $0{ }^{\circ} \mathrm{C}$. Figure 4.12 has no practical application as it was computed assuming no moisture transfer occurred. It is interesting, however, to see the wide range of thermal conductivities possible in a freezing soil, and that the thermal conductivity is influenced primarily by ice content.

The volumetric specific heat of the silica flour, liquid and ice mixture, $\rho c$, was calculated using the following expression:

$$
\rho c=\gamma_{\mathrm{d}}\left(\mathrm{c}_{\mathrm{s}}+4.184 \mathrm{~W}_{\mathrm{u}}+2.10 \mathrm{~W}_{\mathrm{i}}\right)
$$

where,

pc $=$ the volumetric specific heat $\left(\mathrm{J} / \mathrm{m}^{3} \mathrm{C}\right)$,

$\gamma_{\mathrm{d}}=$ the dry density of the silica flour $\left(1330 \mathrm{~kg} / \mathrm{m}^{3}\right)$, 
$\mathrm{c}_{\mathrm{s}}=$ the mass specific heat of the silica flour $(0.837 \mathrm{~J} / \mathrm{g} \mathrm{C})$,

$4.184=$ the mass specific heat of water $(\mathrm{J} / \mathrm{g} \mathrm{C})$,

$\mathrm{W}_{\mathrm{u}}=$ the gravimetric water content (dec.),

$2.10=$ the mass specific heat of ice $(\mathrm{J} / \mathrm{g} \mathrm{C})$, and

$\mathrm{W}_{\mathrm{i}}=$ the gravimetric ice content (dec.).

Figure 4.13 shows the volumetric specific heat for a range of unfrozen water contents computed using equation 4.2 without the ice content term. In the frozen zone, the computation of volumetric specific heat is obtained by including the mass specific heat of ice term in equation 4.2.

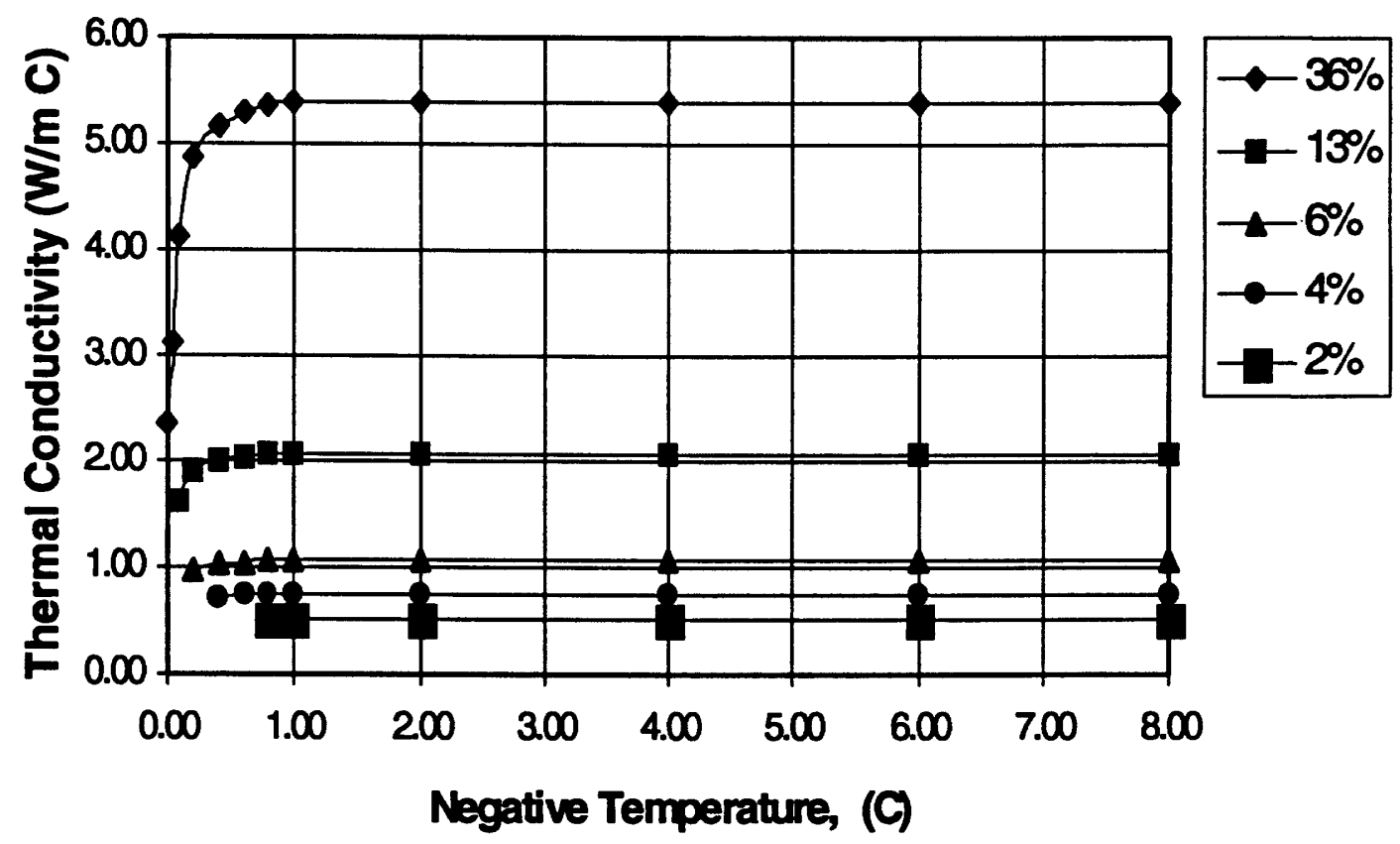

Figure 4.12 Thermal Conductivity for Frozen Silica Flour for Different Initial Gravimetric Water Contents and Neglecting Mass Transfer During Freezing 


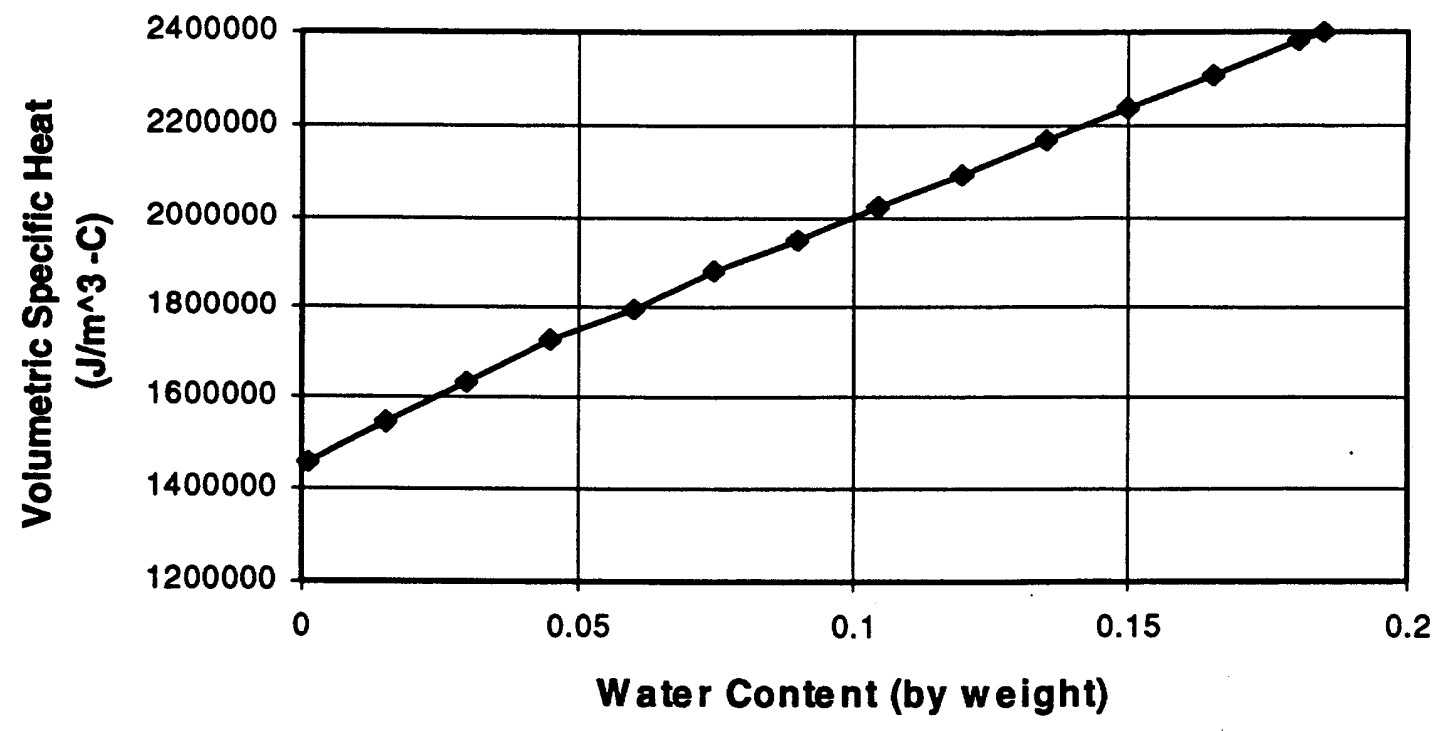

Figure 4.13 Volumetric Specific Heat in Unfrozen Soil 


\section{CHAPTER 5 PRESENTATION OF RESULTS}

\subsection{Introduction}

This chapter presents the results of modelling the Jame (1980) laboratory freezing test data. In addition, the results obtained during calibration of the silica flour hydraulic properties are presented for discussion in Chapter 6.

\subsection{Results of Modelling Jame (1980) Laboratory Data}

The silica flour soil property functions and equations were incorporated into the computer program either as part of a data input file, or as part of a programming subroutine or function modification. To model the freezing tests conducted by Jame (1980) a cold end temperature boundary condition algorithm had to be added to the computer code because the temperatures at the cold end decreased from initial conditions to the prescribed cold end temperature over a period of 0.5 to 4 hours. SoilCover (MEND, 1993) presently does not allow hourly input data. The cold end temperature boundary conditions for each of the tests are given in Figure 5.1.

A finite element grid consisting of 31 nodes with even $1 \mathrm{~cm}$ spacings was used in all of the test verifications. A linear finite element was assumed with two Gauss points in each 
element. The system was considered to have converged if the suctions and temperatures did not change by more than $1 \%$ between successive iterations. Convergence was obtained at every time step in all tests. The Crank - Nicholson central difference time stepping routine scheme was used in the SoilCover program, and times steps were allowed to vary from 4 seconds to 1000 seconds. A time step control parameter limiting the change in time steps to a maximum of $4 \%$ between successive time steps was used. The average time to simulate a 72 hour freezing test was about 15 minutes. The first day took about 10 minutes to simulate, the second day took about 3 minutes to simulate, while the third day took about 1 minute to simulate. In general, the time steps became much larger as the system approached steady state. Finally, since the experiments performed by Jame (1977) were on a horizontal column of soil, the gravity term in the mass transfer equation was turned off in the computer program code.

As discussed in section 4.3, the precise saturated coefficient of permeability and ice impedance factors were not known prior to modelling. For this reason, modelling was carried out using a saturated coefficient of permeability ranging over one half an order of magnitude from $4.5 \times 10^{-5} \mathrm{~cm} / \mathrm{s}$ to $9.5 \times 10^{-5} \mathrm{~cm} / \mathrm{s}$. Initially, an ice impedance factor was not applied. The results of the modelling using a saturated coefficient of permeability of $7.0 \times 10^{-5} \mathrm{~cm} / \mathrm{s}$ are illustrated in Figures 5.2 through 5.4 for the Jame (1980) tests 1 to 3 respectively. Results obtained during the calibration of soil hydraulic properties are presented in the next section. 

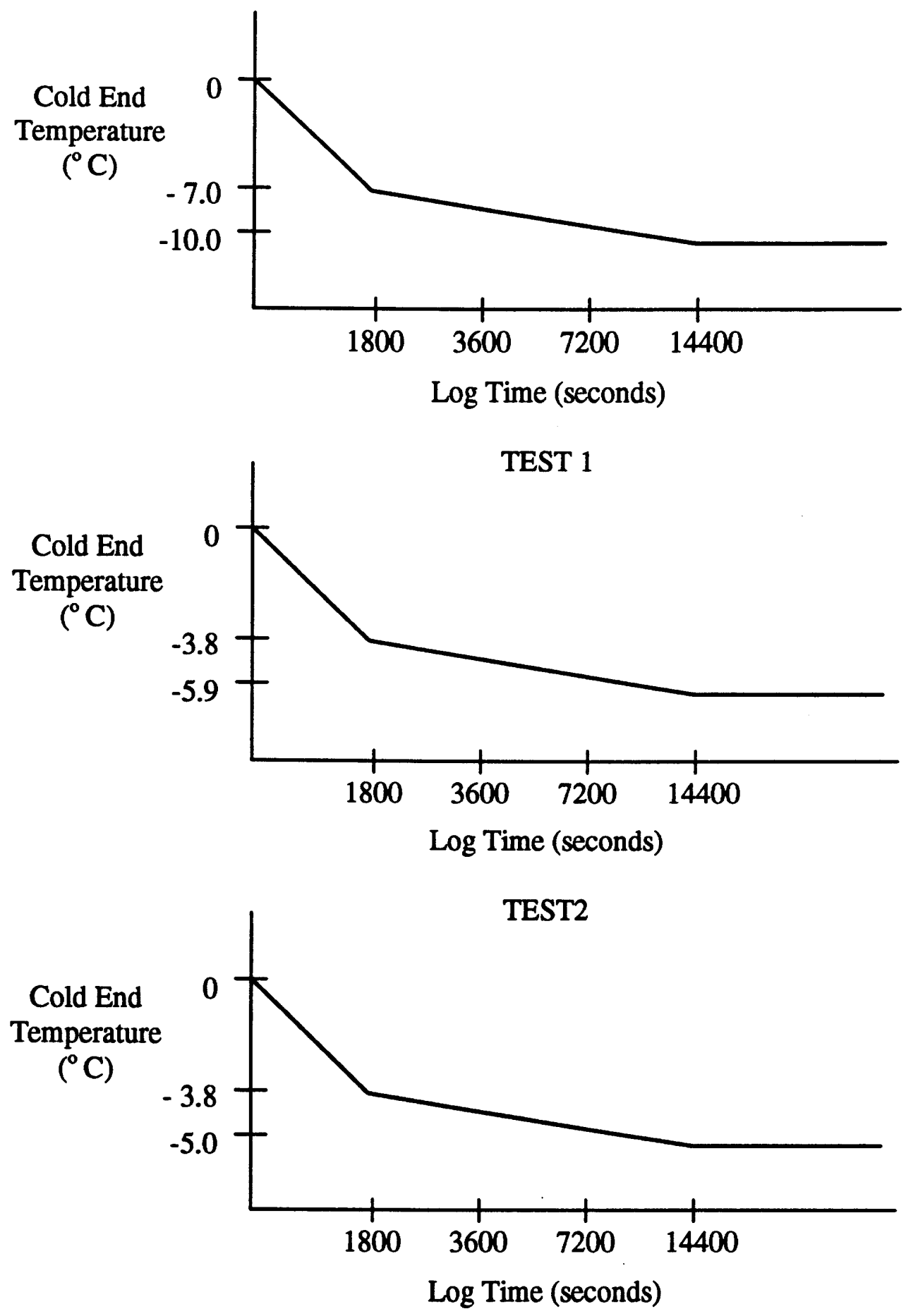

TEST 3

Figure 5.1 Cold End Temperature Boundary Conditions for Tests 1 - 3 (Jame, 1980) 

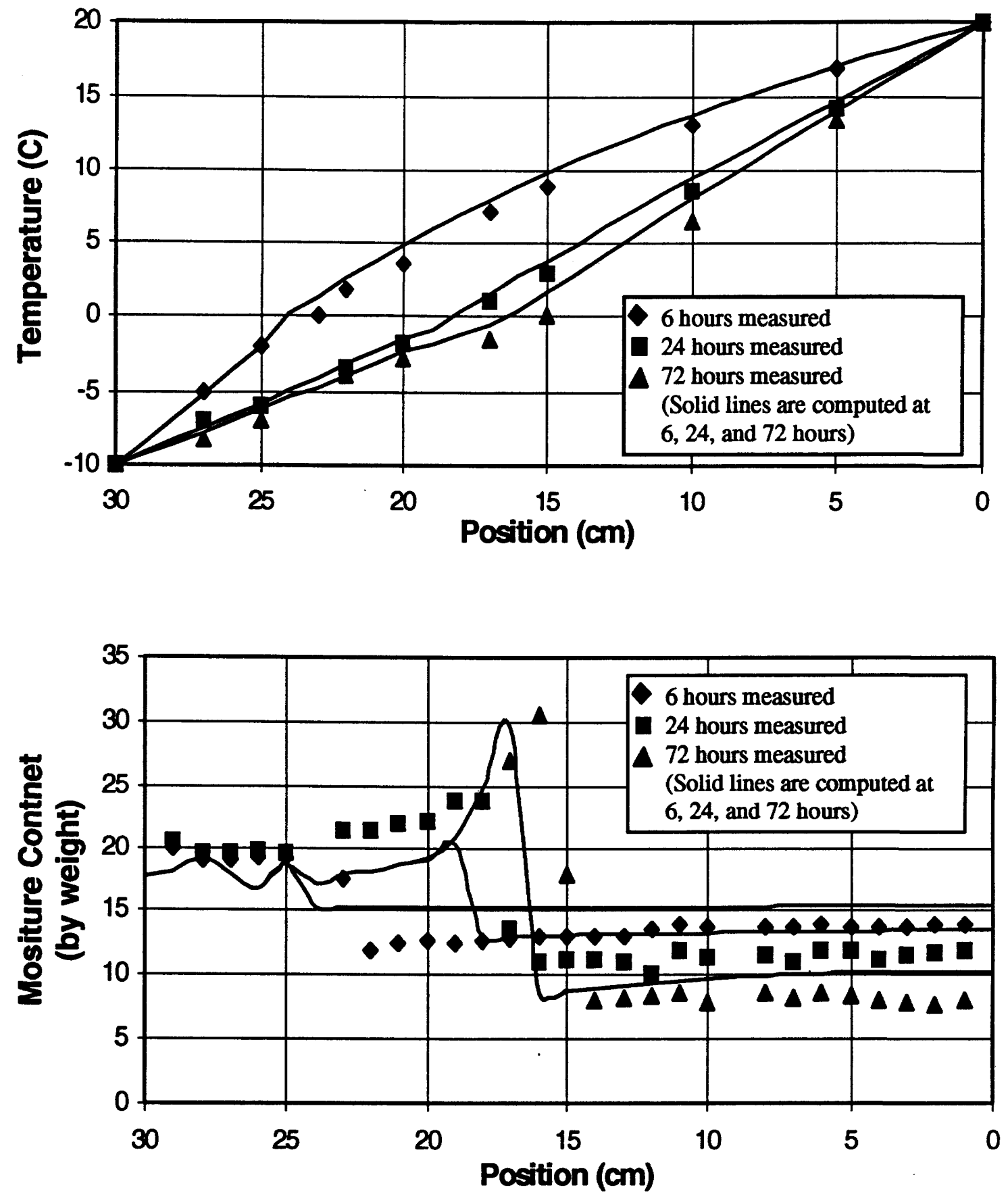

Figure 5.2 Modelling Results of Test 1 

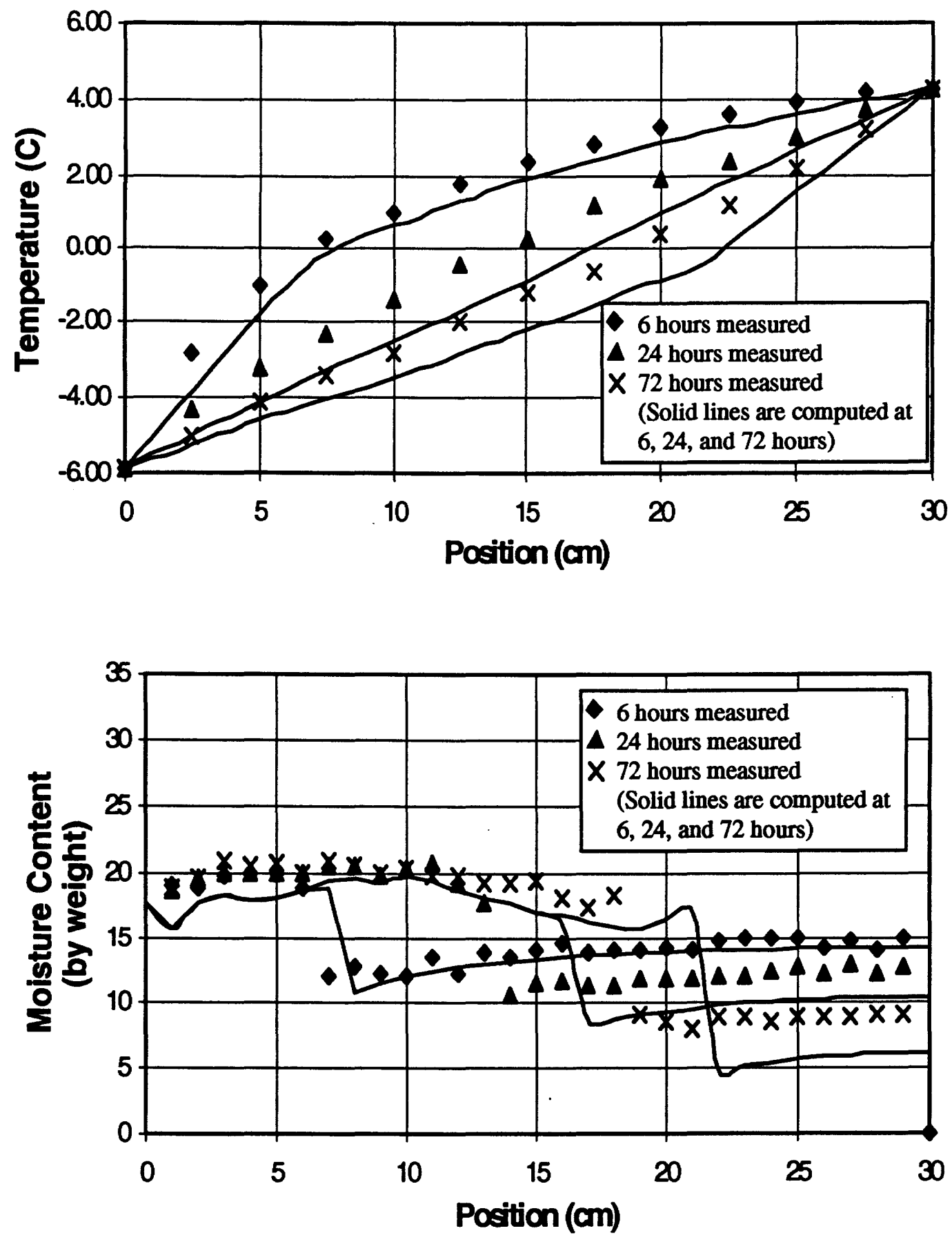

Figure 5.3 Modelling Results of Test 2 

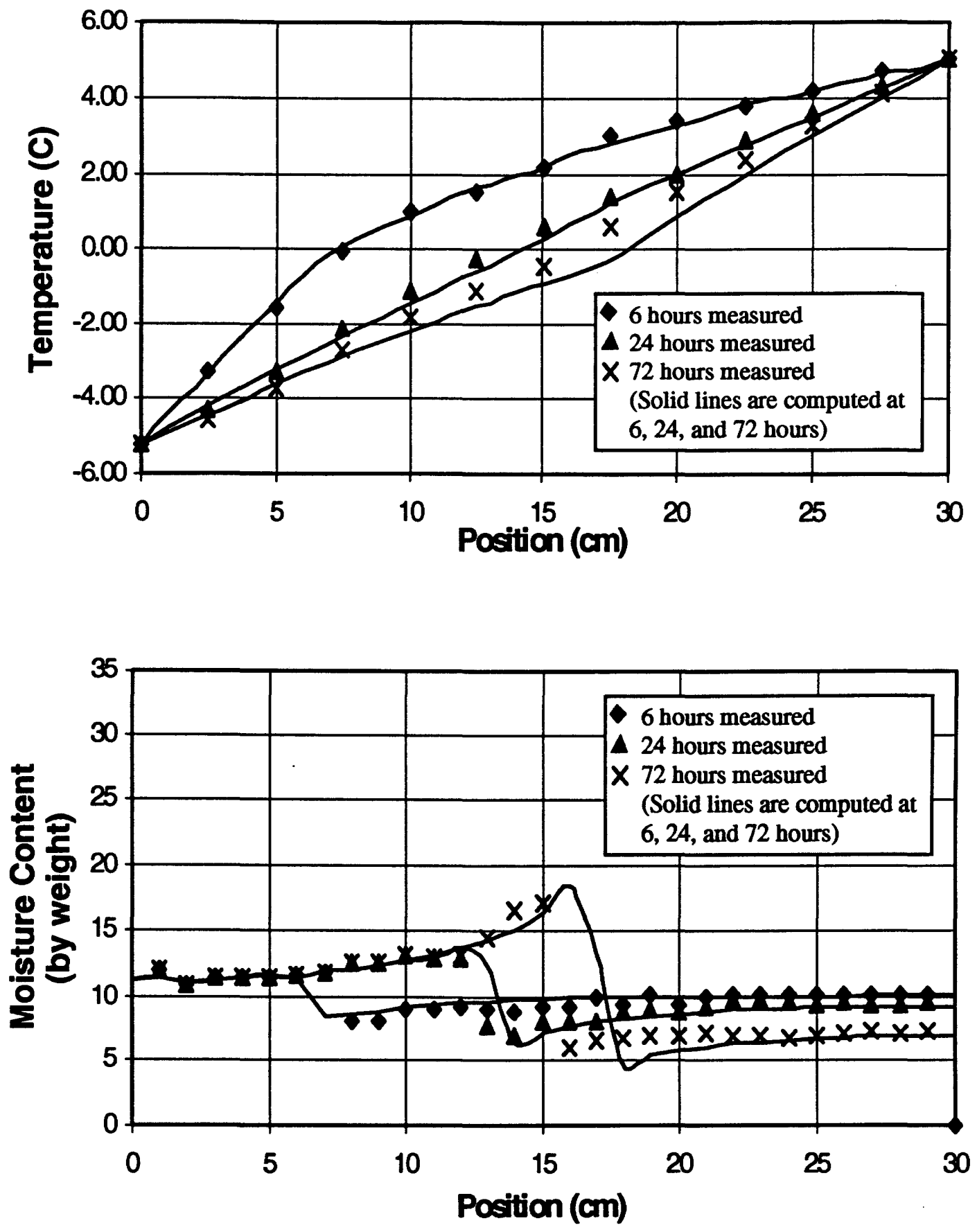

Figure 5.4 Modelling Results of Test 3 


\subsection{Calibration of Hydraulic Properties}

In section 4.3, mention was made of the fact that the saturated coefficient of permeability was experimentally determined to be within the range of $2.5 \times 10^{-4} \mathrm{~cm} / \mathrm{s}$ at a porosity of 0.52 , to $3.0 \times 10^{-5} \mathrm{~cm} / \mathrm{s}$ at a porosity of 0.48 . The modified Tempe Cell test for the material used in this study indicated the porosity to be about 0.51 . As a result, three different values for the saturated coefficients of permeability were selected for testing. All values were within half an order of magnitude of each other. It was also noted previously that the measured soil water characteristic curve did not account for suctions above $100 \mathrm{kPa}$. In hindsight, additional experimental testing should have been carried out to determine the volumetric water contents at higher values of suctions. Since this was not done, three different soil water characteristic curves were used for comparison purposes in this study. Finally, a range of ice impedance factors were applied to the permeability values to determine their significance and to obtain the correct empirical constant which could be used to calibrate the computer model for accurate simulation of the data presented by Jame (1980).

The saturated coefficients of permeability used in this study were $4.5 \times 10^{-5} \mathrm{~cm} / \mathrm{s}, 7.0 \mathrm{x}$ $10^{-5} \mathrm{~cm} / \mathrm{s}$, and $9.5 \times 10^{-5} \mathrm{~cm} / \mathrm{s}$. The three different soil water characteristic curves used are shown in Figure 5.5a. The corresponding relative permeability functions obtained using the Fredlund et al. (1994) equations are given in Figure 5.5b. The three different ice impedance factors are shown in Figure 5.6.

The saturated coefficients of permeability and soil water characteristic curves were chosen in such a way that they would adequately represent the measured soil property. The ice impedance factors were chosen such that they ranged from zero impedance to a three order of magnitude drop in permeability at a volumetric ice content of 0.25 . Zero ice impedance would imply that the permeability given by the permeability versus suction relationship for an unfrozen soil would also apply in a frozen soil. A three order of magnitude drop in permeability impedance factor would be similar to that which Jame (1980) applied for simulating the experimental data in his study. In this study, every effort 
was made to avoid unreasonable adjustments of material parameters in order to obtain the desired results. Table 5.1 summarizes the numerical simulations using the various soil properties.

Table 5.1 Summary of Test Conditions Used in Calibration of Hydraulic Properites

\begin{tabular}{|c|c|c|c|c|}
\hline $\begin{array}{c}\text { Test Record } \\
\text { Number }\end{array}$ & $\begin{array}{c}\text { Jame Test } \\
\text { Number }\end{array}$ & SWC Type & $\begin{array}{c}\text { ksat. } \times 10^{-5} \\
(\mathrm{~cm} / \mathrm{s})\end{array}$ & $\begin{array}{c}\text { Impedance Factor } \\
\text { Empirical Constant }\end{array}$ \\
\hline JT 102 & 1 & 1 & 4.5 & 0 \\
\hline JT 202 & 2 & 1 & 4.5 & 0 \\
\hline JT 302 & 3 & 1 & 4.5 & 0 \\
\hline JT 103 & 1 & 2 & 4.5 & 0 \\
\hline JT 203 & 2 & 2 & 4.5 & 0 \\
\hline JT 303 & 3 & 2 & 4.5 & 0 \\
\hline JT 104 & 1 & 3 & 4.5 & 0 \\
\hline JT 204 & 2 & 3 & 4.5 & 0 \\
\hline JT 304 & 3 & 3 & 4.5 & 0 \\
\hline JT 105 & 1 & 3 & 7.0 & 0 \\
\hline JT 205 & 2 & 3 & 7.0 & 0 \\
\hline JT 305 & 3 & 3 & 7.0 & 0 \\
\hline JT 306 & 3 & 3 & 7.0 & 6 \\
\hline JT 307 & 3 & 3 & 7.0 & 12 \\
\hline JT 108 & 1 & 3 & 9.5 & 0 \\
\hline JT 208 & 2 & 3 & 9.5 & 0 \\
\hline JT 308 & 3 & 3 & 9.5 & 0 \\
\hline
\end{tabular}




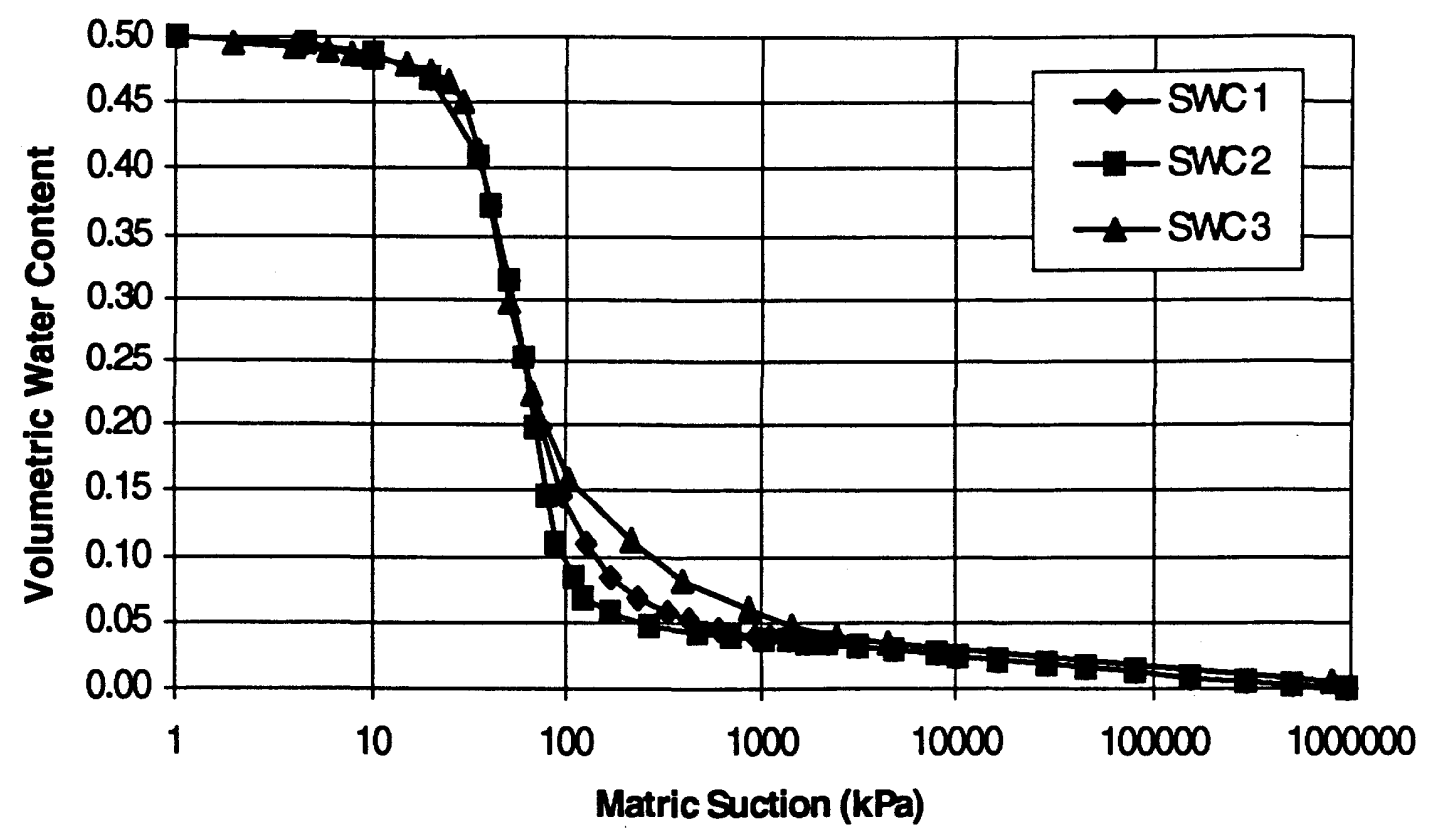

Figure 5.5a Soil Water Characteristic Curves Used In Calibrations

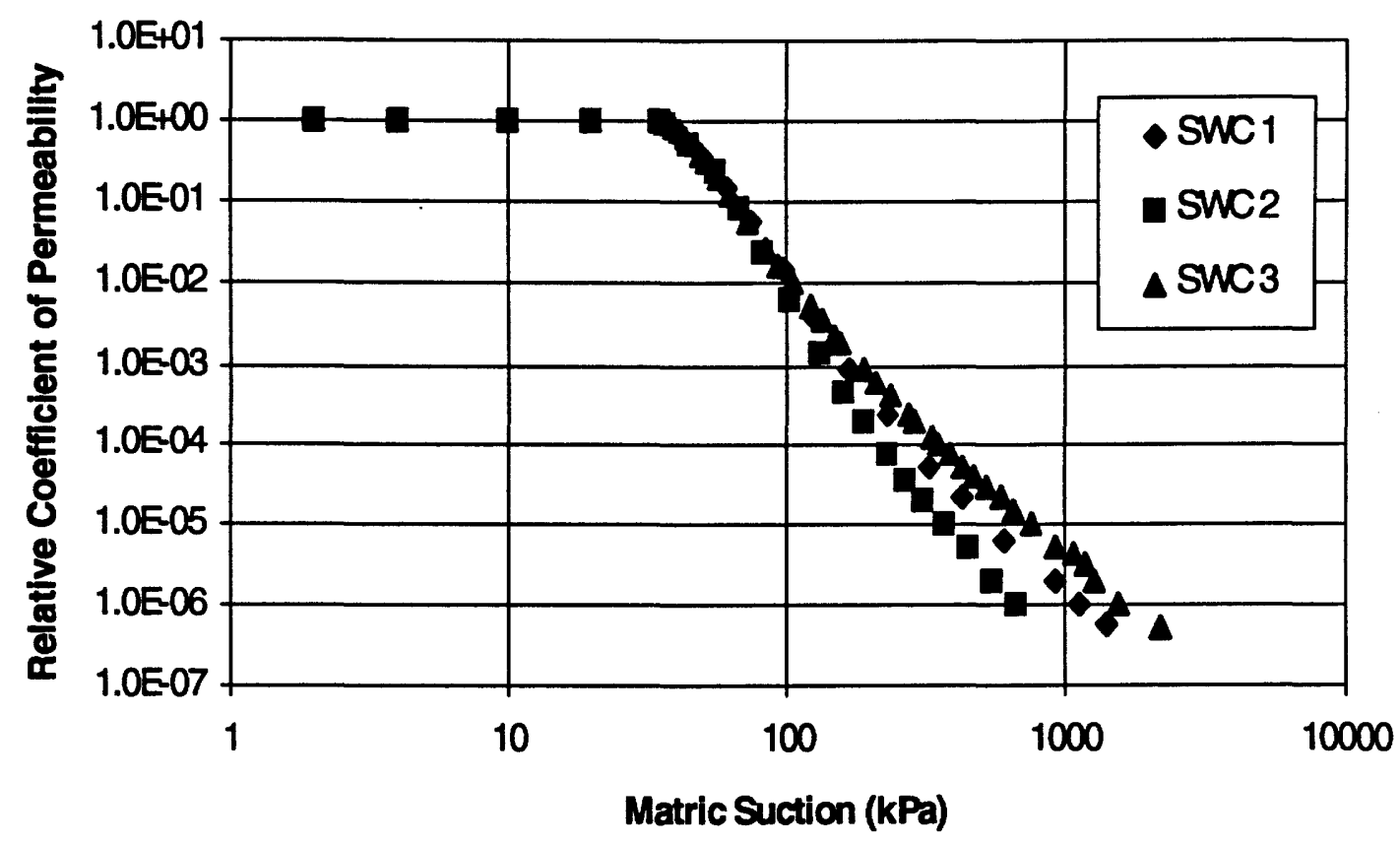

Figure 5.5b Relative Coefficient of Permeability Curves Obtained Using the Fredlund et al. (1994) Equations for the SWC Curves Used in Calibrations 


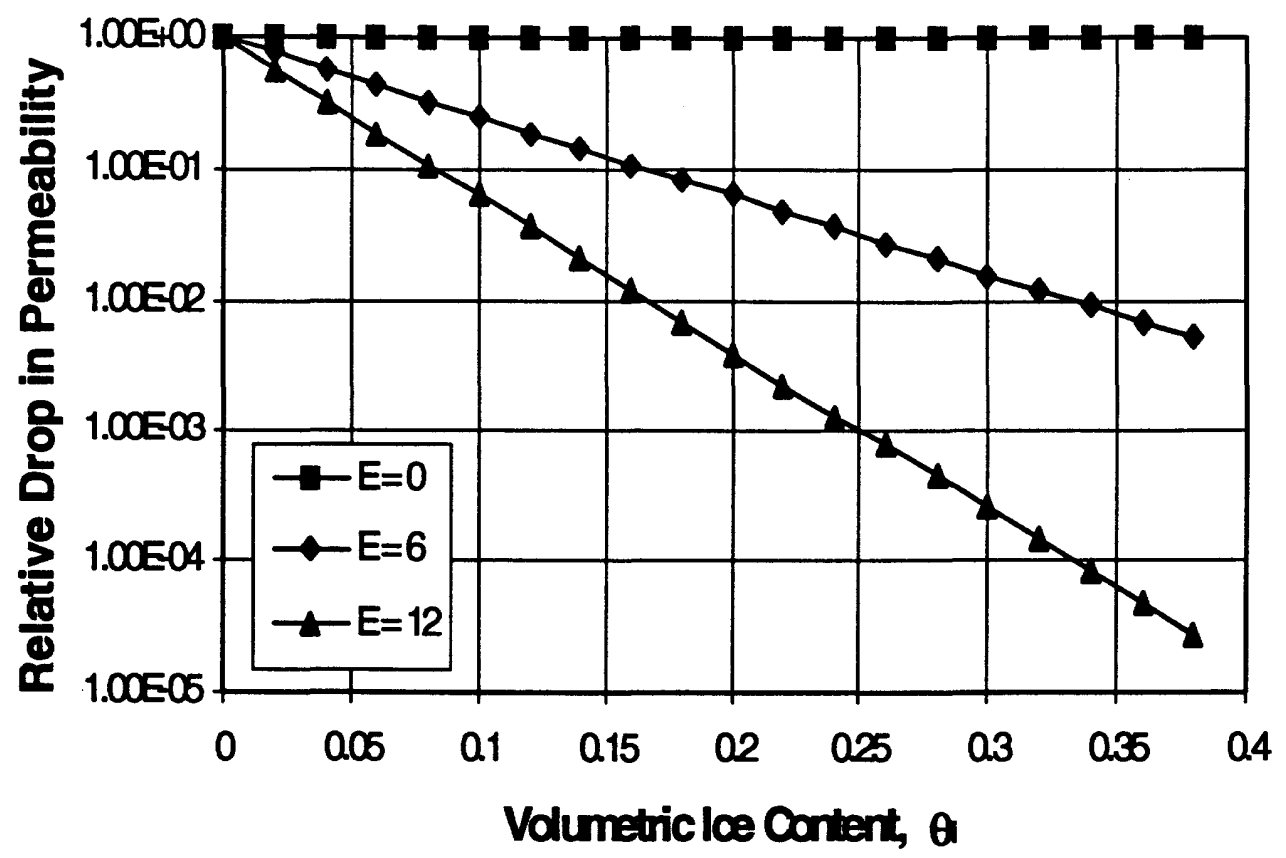

Figure 5.6 Range of Ice Impedance Factors and Relative Magnitude Applied in this Study

Simulations were carried out in the order they appear in table 5.1. Initially, the soil water characteristic curves were varied for each of the Jame (1977) freezing tests with an initial saturated coefficient of permeability of $4.5 \times 10^{-5} \mathrm{~cm} / \mathrm{s}$ and no impedance factor applied. These initial simulations indicated that an impedance factor was likely not necessary. After the initial nine simulations were complete (i.e., Table 5.1) the computed suction and temperature profiles showed that soil type 3 gave the most reasonable agreement with measured results when considering all three freezing tests. Using the soil water characteristic curve shown as soil type 3 , six more simulations were performed to compare the effects of increasing the saturated coefficient of permeability. Once the most reasonable permeability was established at $7.0 \times 10^{-5} \mathrm{~cm} / \mathrm{s}$, additional testing was done to study the effect of adding different ice impedance factors. The results of some of these tests are presented below. Comments and general observations about the results are presented in Chapter 6. 
Figures 5.7 and 5.8 compare the temperatures and total moisture profiles simulated using the three slightly different soil water characteristic curves. These results were obtained for simulation of test 3 , using a saturated coefficient of permeability of $4.5 \times 10^{-5} \mathrm{~cm} / \mathrm{s}$ and no impedance factor.

Figures 5.9 and 5.10 compare the temperature and total moisture profiles simulated with SWC 3 and three different saturated coefficients of permeability. Again, these results were obtained for simulation of test 3 , using no impedance factor.

Figures 5.11 and 5.12 compare the temperature and total moisture profiles simulated with three different permeability ice impedance factors. These results were obtained for simulation of test 3, using SWC 3, and a saturated coefficient of permeability of $7.0 \times 10^{-5}$ $\mathrm{cm} / \mathrm{s}$. 


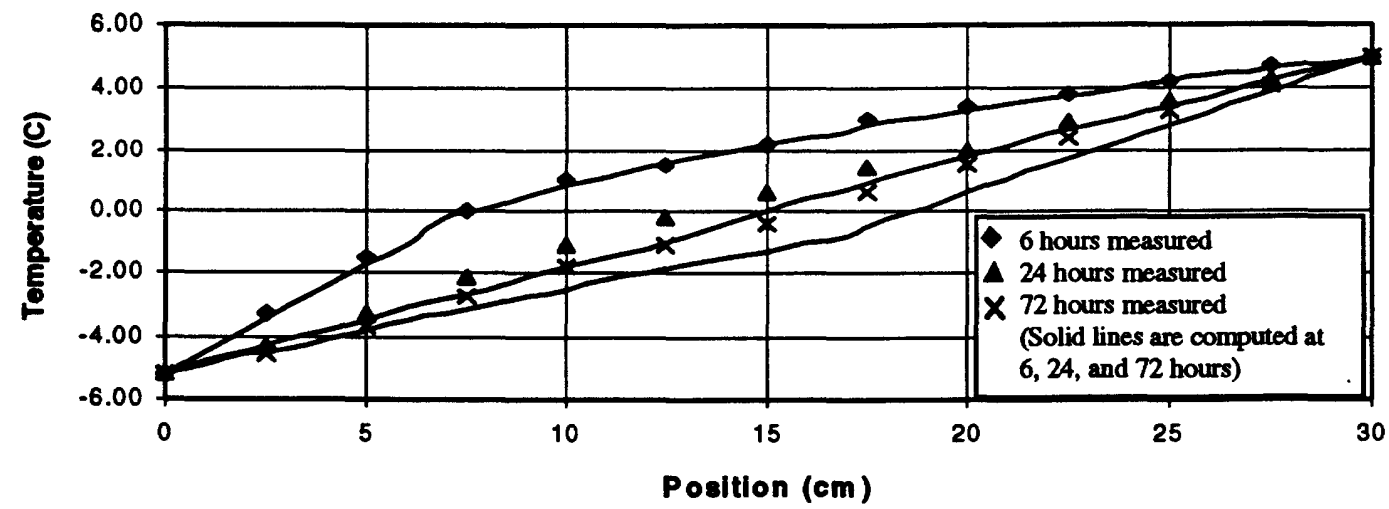

Figure 5.7a SWC 1

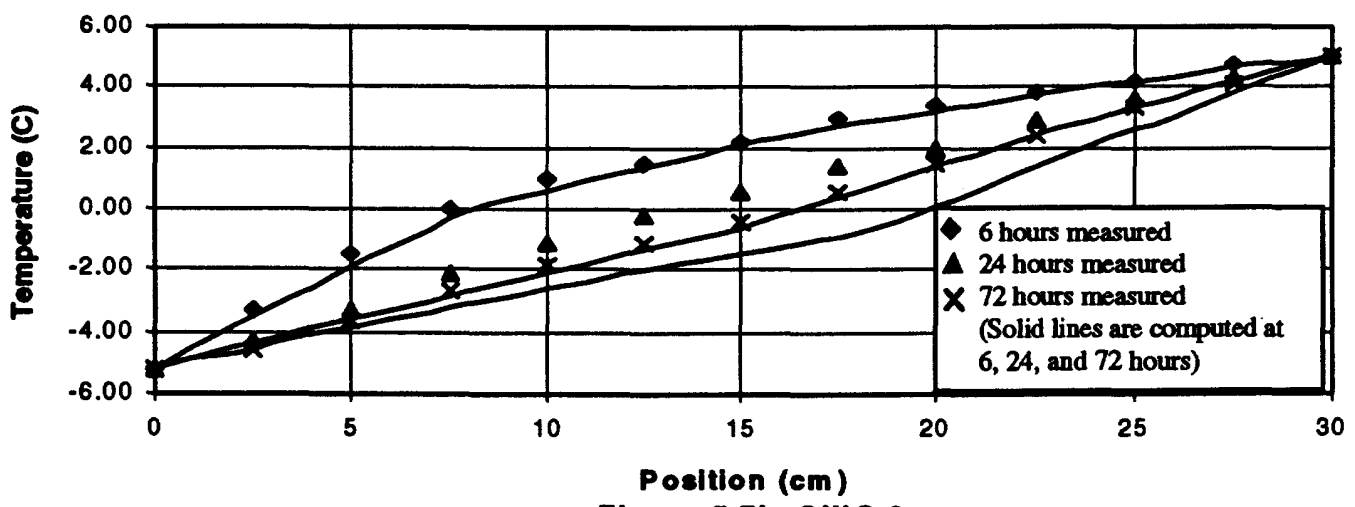

Flgure 5.7b SWC 2

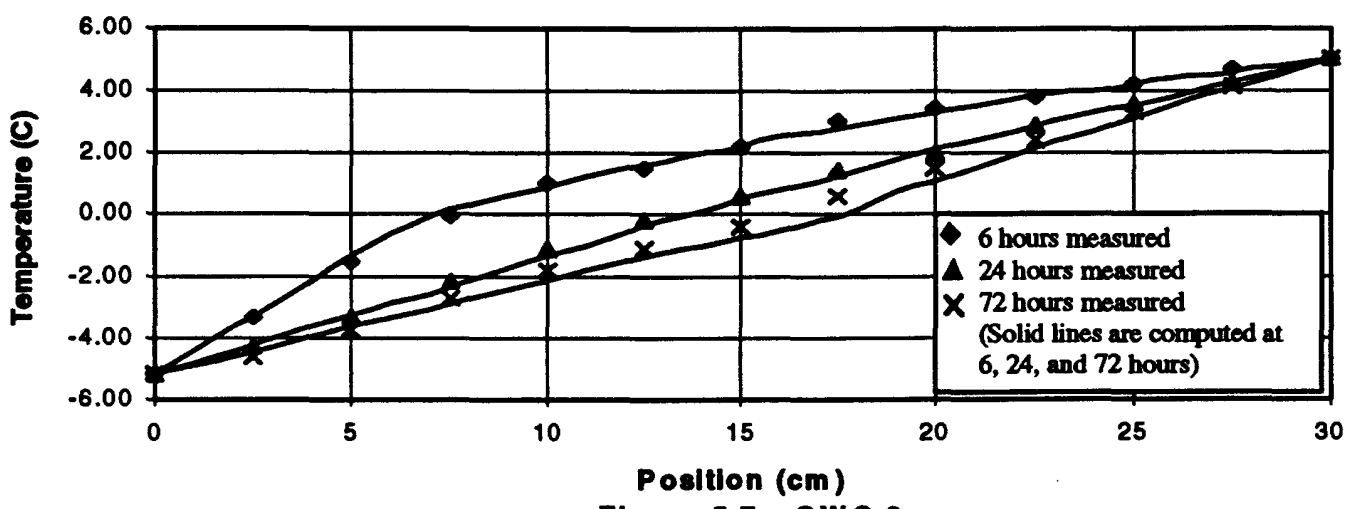

Figure 5.7e SWC 3

Figure 5.7 Comparison of Temperature Profiles for Test 3 Using Three Different Soil Water Characteristic Curves, ksat $=4.5 \times 10^{-5} \mathrm{~cm} / \mathrm{s}$, and No Impedance Factor 


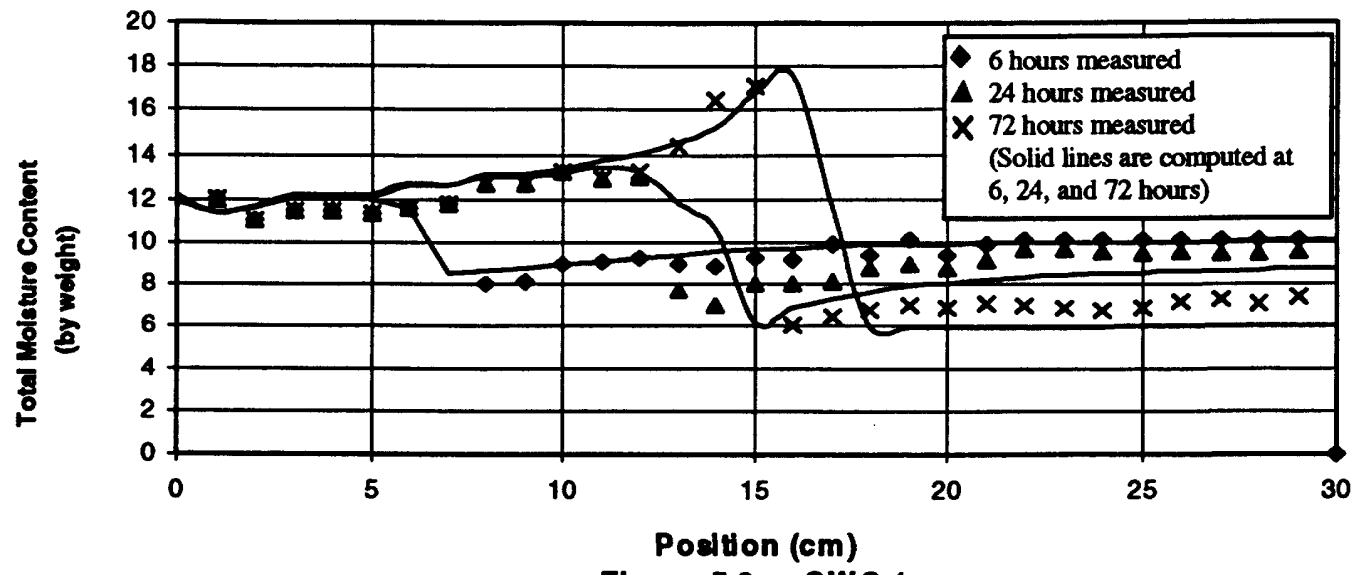

Figure 5.8a SWC 1

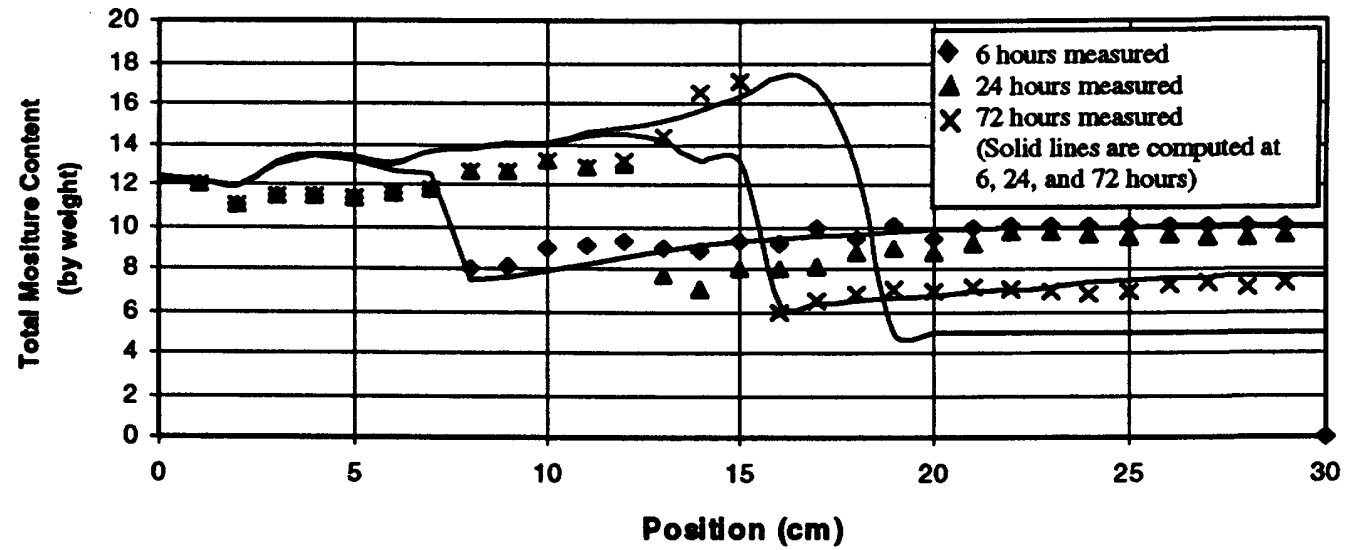

Figure 5.8b SWC 2

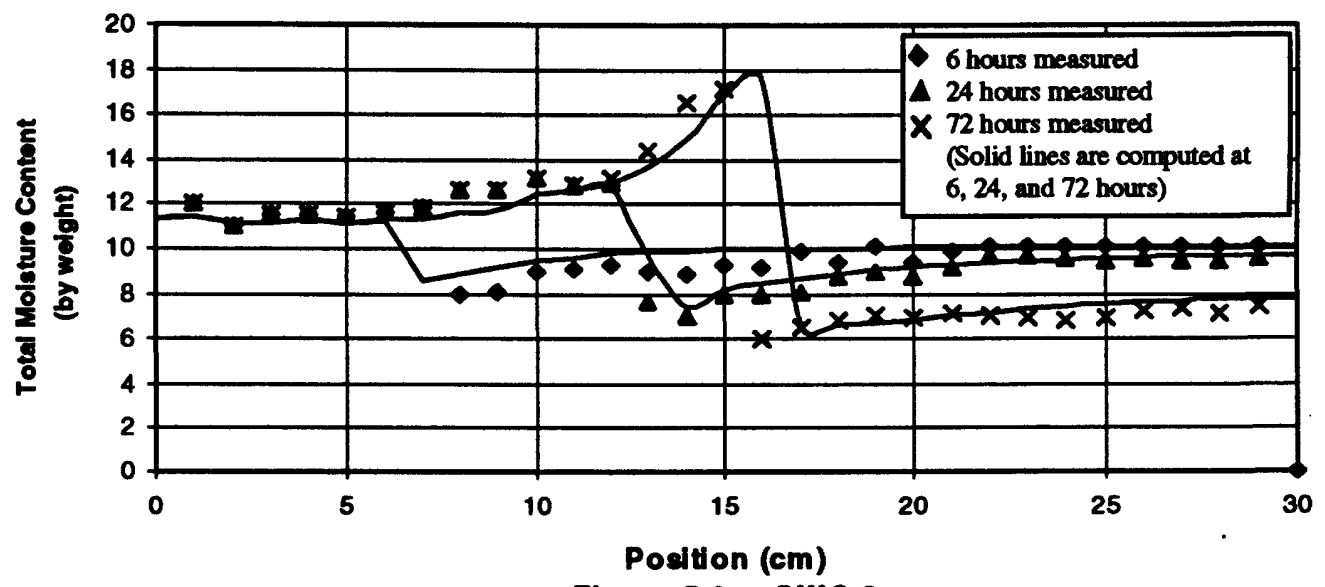

Flgure 5.8C SWC 3

Figure 5.8 Comparison of Moisture Profiles for Test 3 Using Three Different Soil Water Characteristic Curves, ksat $=4.5 \times 10^{-5} \mathrm{~cm} / \mathrm{s}$, and No Impedance Factor 


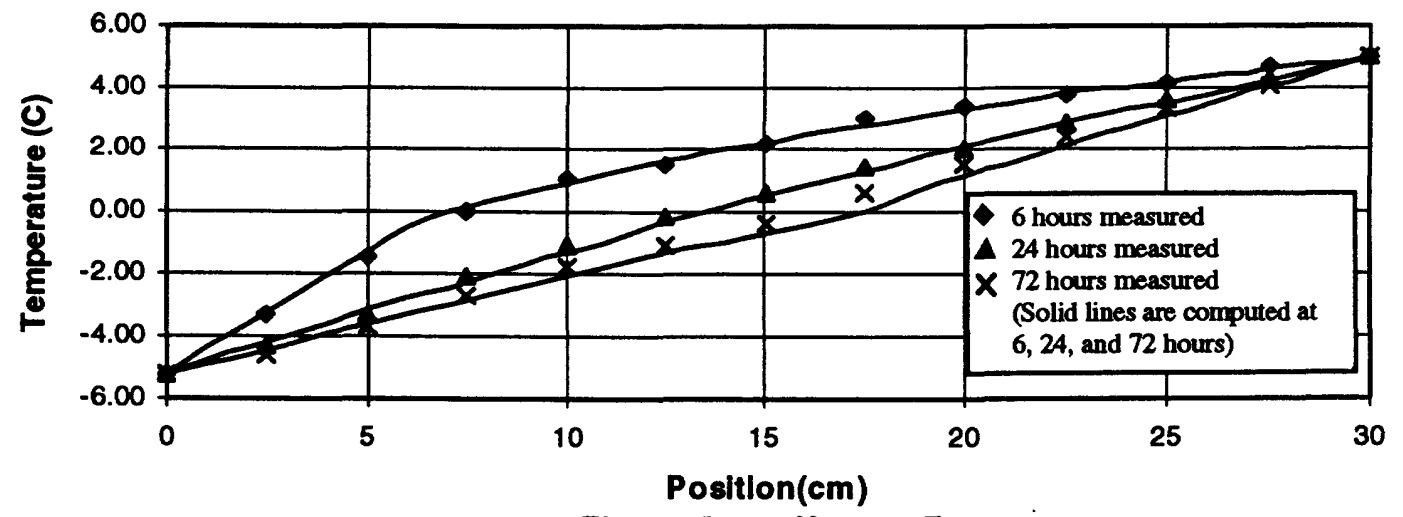

Figure 5.9a $\quad K s=4.5 E-5$

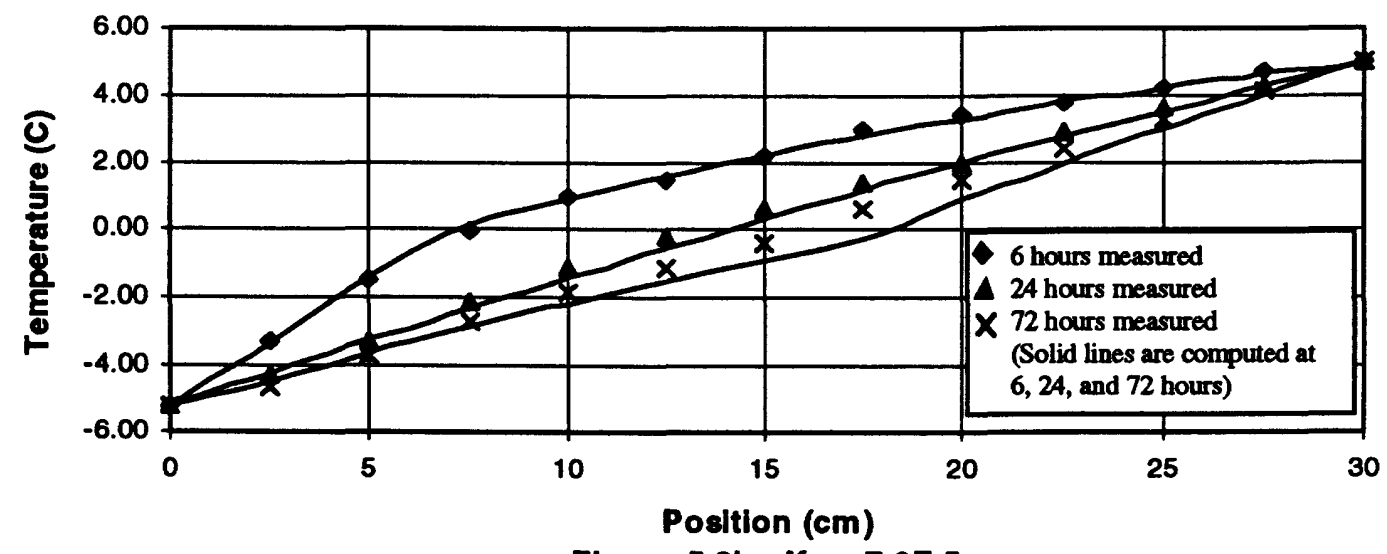

Figure 5.9b $\quad K 8=7.0 E-5$

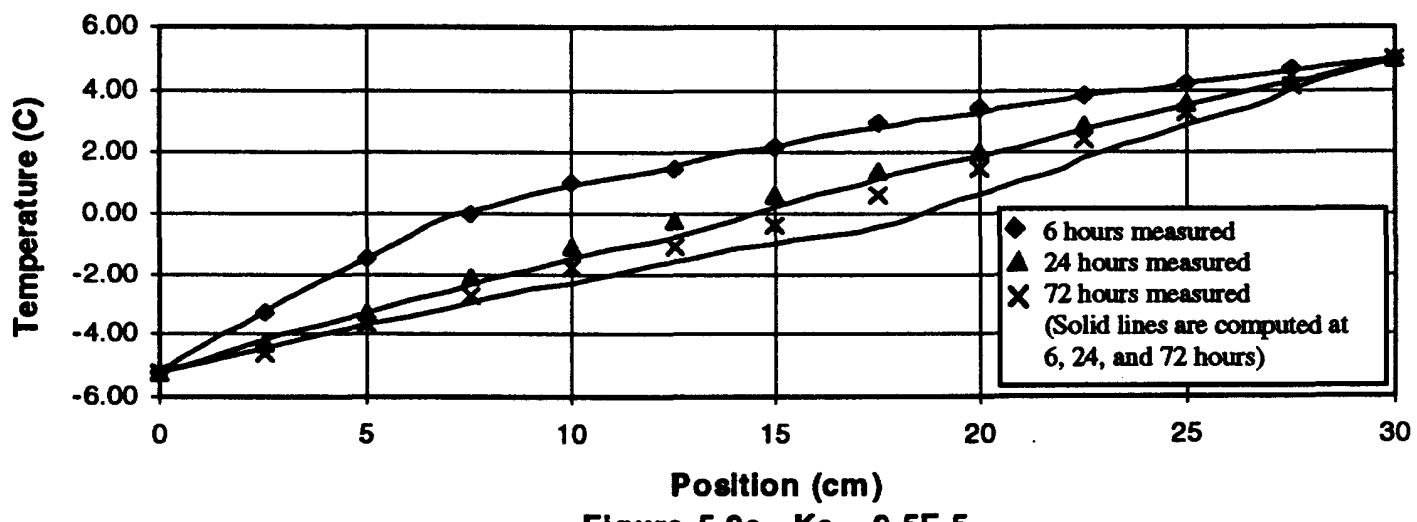

Figure $5.9 \mathrm{C} \quad \mathrm{Ks}=\mathbf{9} .5 \mathrm{E}-5$

Figure 5.9 Comparison of Temperature Profiles for Test 3 Using Three Different Saturated Coefficients of Permeability, SWC 3, and No Impedance Factor 


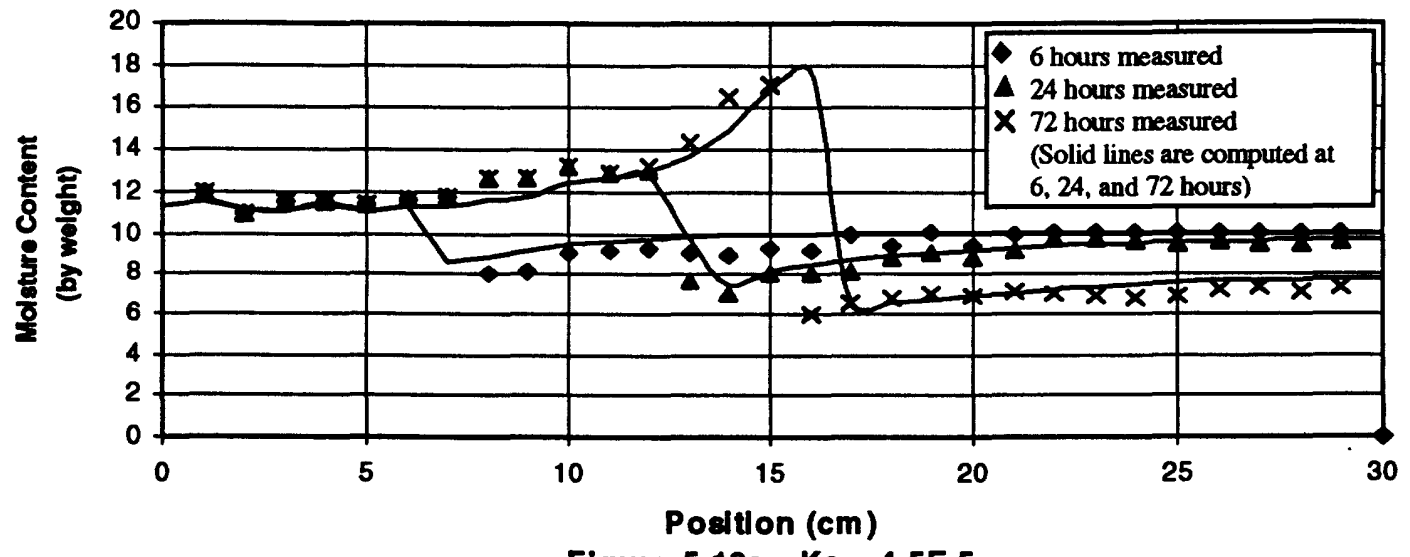

Figure 5.10a $\quad K s=4.5 E-5$
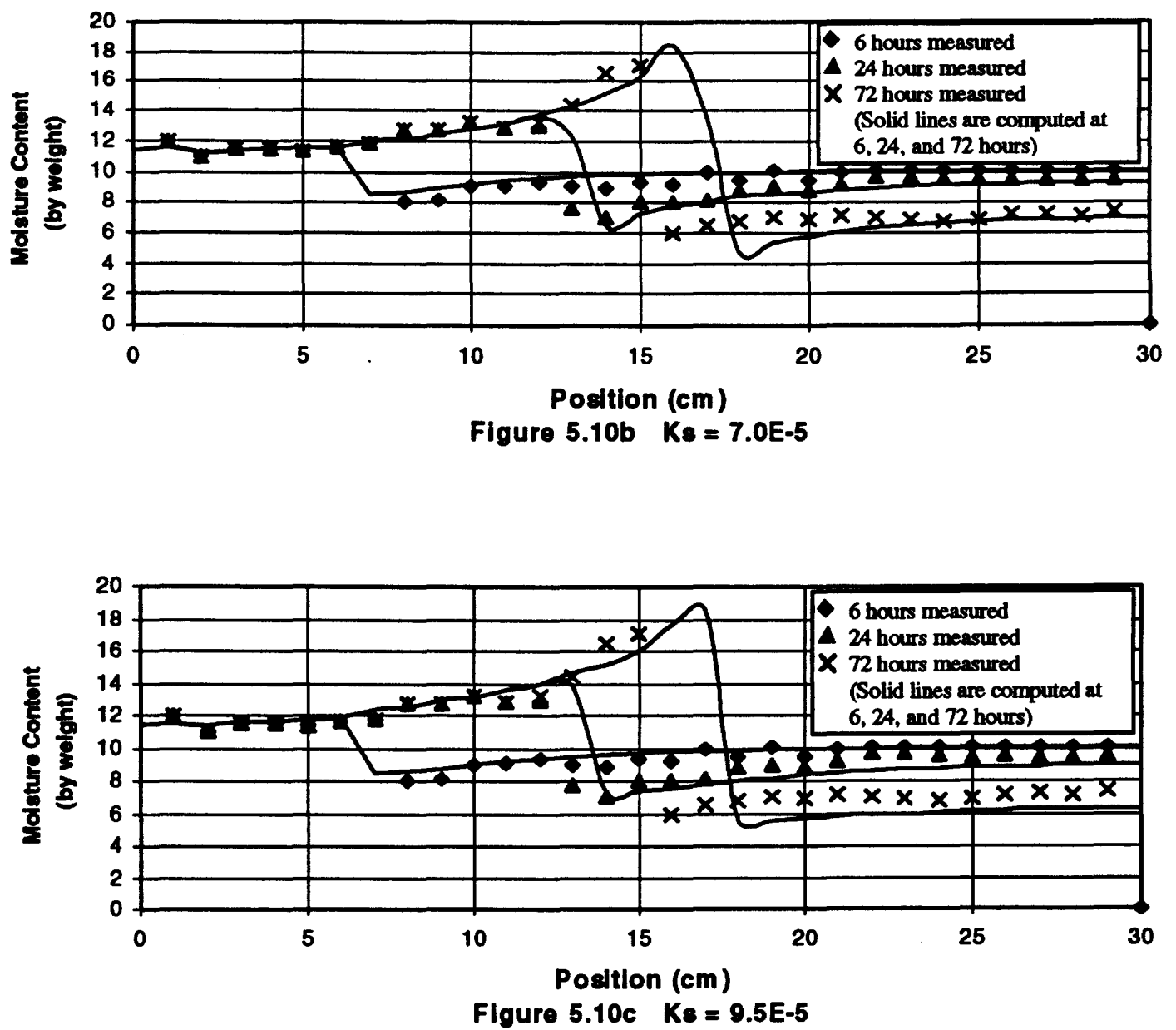

Figure 5.10 Comparison of Moisture Profiles for Test 3 Using Three Different Saturated Coefficients of Permeability, SWC 3, and No Impedance Factor 

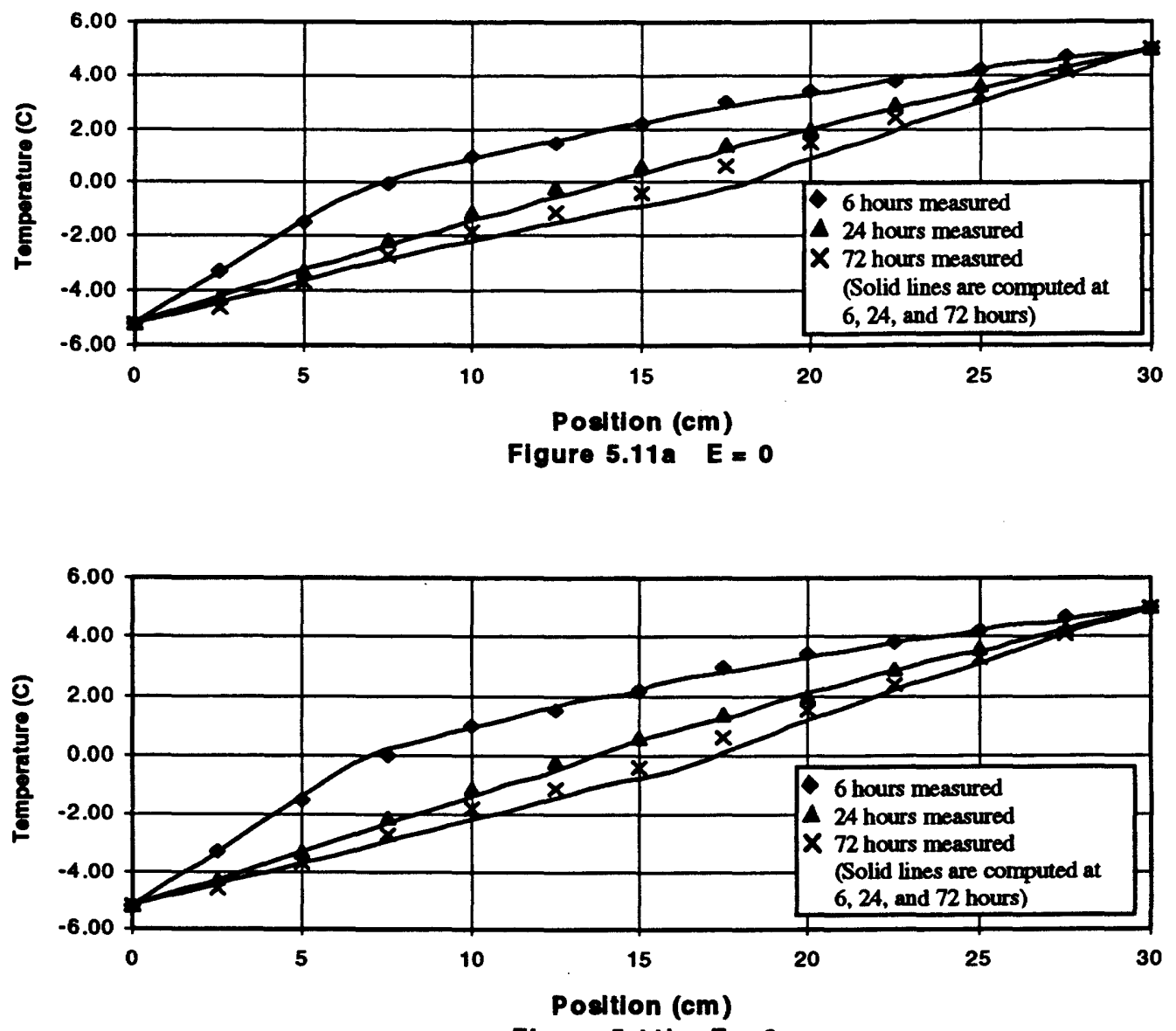

Figure 5.11b $E=6$

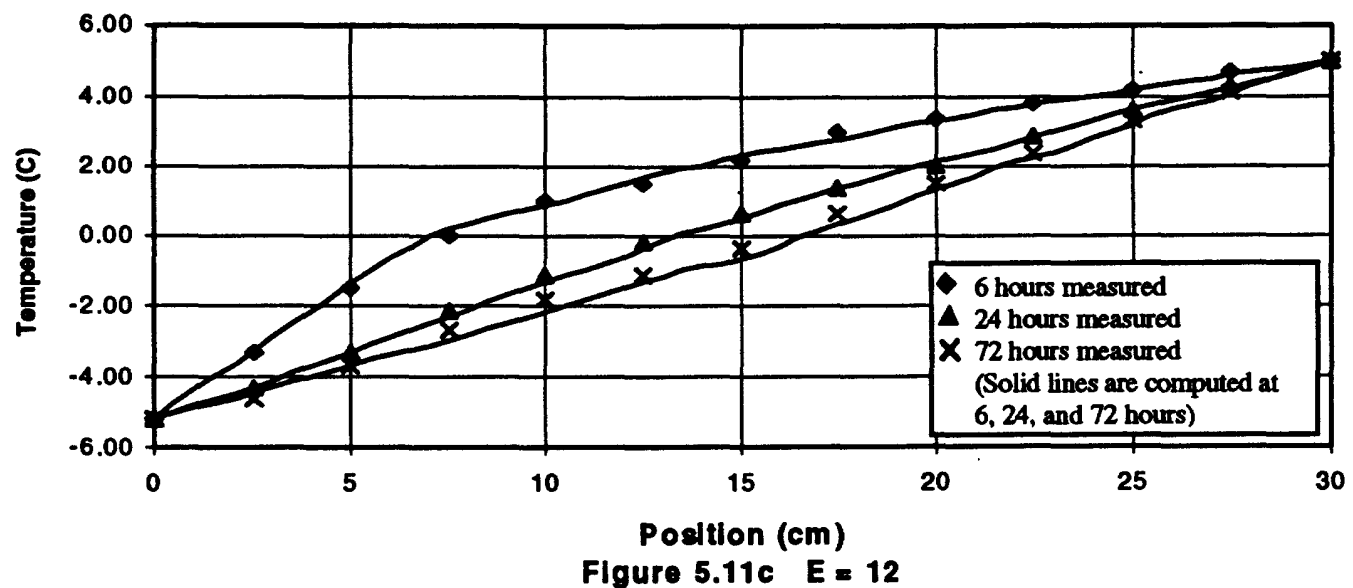

Figure 5.11 Comparison of Temperature Profiles for Test 3 Using Three Different Ice Impedance Factor Coefficients, SWC 3, and Ksat $=7.0 \times 10^{-5} \mathrm{~cm} / \mathrm{s}$ 


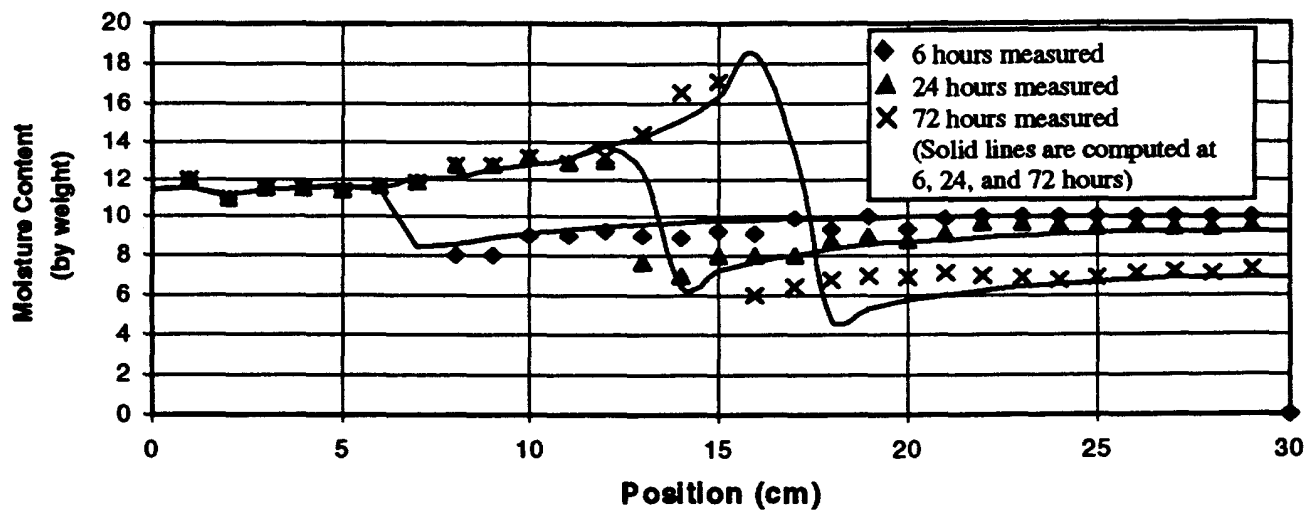

Flgure 5.12a $E=0$

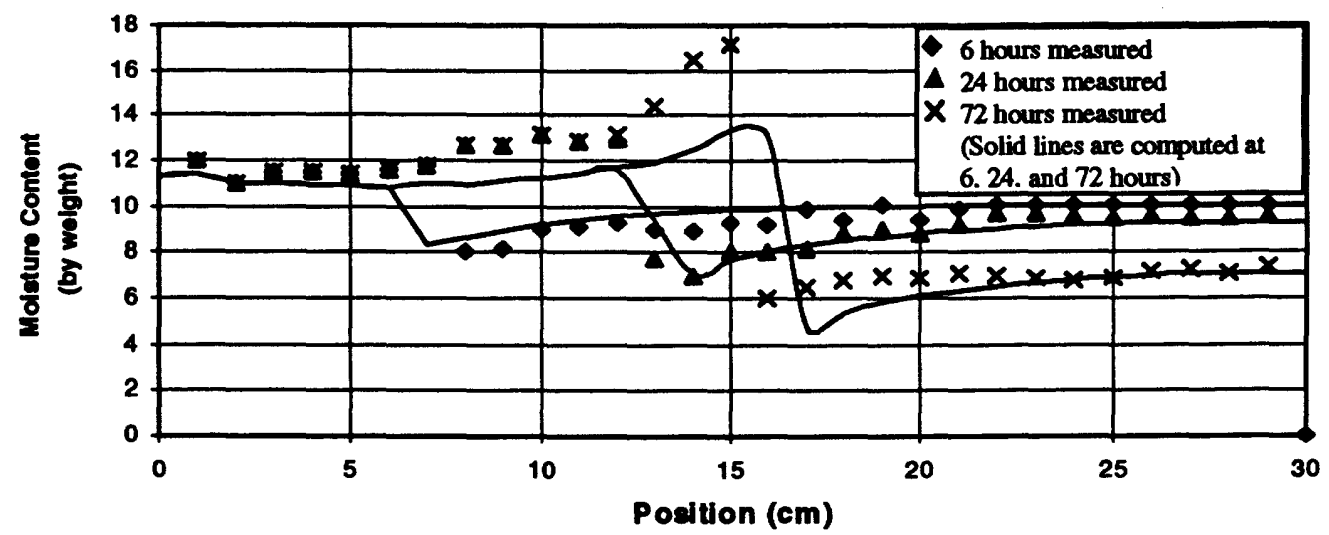

Figure $5.12 b \quad E=6$

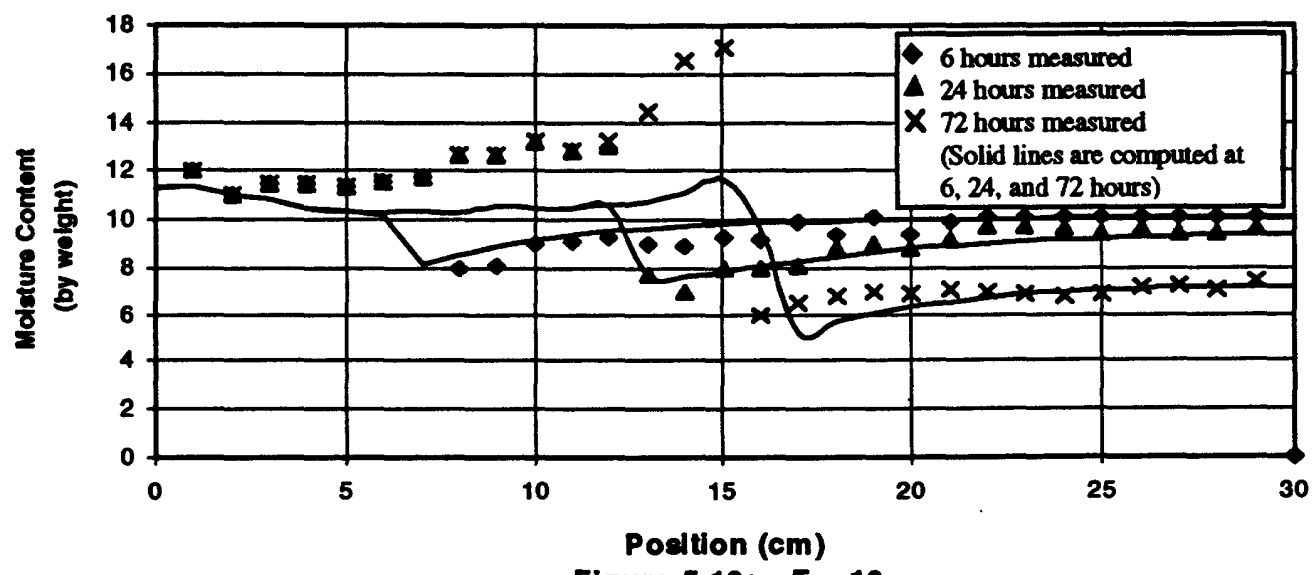

Figure $5.12 c E=12$

Figure 5.12 Comparison of Moisture Profiles for Test 3 Using Three Different Ice Impedance Factor Coefficients, SWC 3, and Ksat $=7.0 \times 10^{-5} \mathrm{~cm} / \mathrm{s}$ 


\section{CHAPTER 6 DISCUSSION OF MODELLING RESULTS}

\subsection{Introduction}

In Chapter 3 the theoretical framework for heat and mass transfer in freezing soils was presented. The computer program SoilCover (MEND, 1993) was modified to provide a numerical solution for the proposed theory. In Chapter 5 the results of the computer simulations for heat and mass flow in freezing soils under controlled were presented. The discussions in this chapter address several issues including the results of the laboratory data simulation program, and the advantages and limitations of the numerical model.

\subsection{Advantages and Limitations of the Revised Numerical Solution}

This section discusses some of the advantages and limitations of the revised heat and mass transfer model. The advantages deal mainly with how the phase change theory was incorporated into an existing non-freezing computer model. The limitations deal mainly with the assumptions used in developing the revised model. In addition, specific reasons for minor discrepancies between measured and computed results are discussed at the end of section 6.3 for the laboratory data modelling program.

In a non-freezing, unsaturated soil, the heat and mass transfer equations can be coupled by the water vapour pressure term which appears in each equation (Wilson, 1990). The 
dominant coupling term between the heat and mass transfer continuity relationships for freezing soils is the volumetric ice content. In the freezing case mass transfer continuity relationship, water that changes phase to ice is no longer considered free to transfer through the system. Furthermore, water that changes phase instantly introduces latent heat into the system at the point of phase change. The original SoilCover (MEND, 1993) program for non-freezing conditions was written so that the dependent variables used in the solution scheme are matric suction and temperature which are coupled together by the vapour pressure of the free water. The addition of the volumetric ice content variable causes problems to the numerical solution because the system of equations becomes indeterminate (i.e., there are two equations and three unknowns).

A common approach to the solution for this problem is to estimate an ice content for each new time step so that the corresponding latent heat of phase change and moisture sink quantities can be used to balance the heat and mass continuity equations over the next time step. This was the approach taken by various researchers who developed soil freezing models. For example, Jame (1977) estimated a new ice content for each time step by computing the heat transfer over the next time step assuming no moisture flux. A change in ice content was then back calculated based on the difference in unfrozen water contents between the current temperature and the estimated new temperature. The change in ice content was then applied to the main coupled heat and mass transfer equations and the procedure continued until convergence was achieved. This proved to be a cumbersome approach which required long computing times with convergence difficulties as the mass transfer component was neglected in the initial estimate of change in ice content.

In the revised numerical model, latent heat of phase change is applied to the heat transfer equation intrinsically because the mass transfer equation is incorporated in the heat transfer equation. There is no need to guess a change in ice content outside of the main solution algorithm and, as a result, the computing time is greatly reduced and convergence becomes a minor problem that is easily rectifiable by adjusting time step or convergence 
criteria. The actual change in ice content over the previous time step is calculated at the end of each iteration so that the soil thermal and hydraulic properties can be modified between iterations. Once the system has converged, the current change in ice content over the previous time step is computed and added to an total nodal ice content array.

The limitations of the revised model relate primarily to the assumptions made for the theoretical development. Convective heat transfer was omitted from the heat transfer equation. This is a reasonable assumption when modelling freezing and thawing in compacted fine materials. However, it is a questionable assumption when modelling freezing and thawing in less dense, coarser materials especially if high liquid fluxes are anticipated (i.e., from snow melt infiltration, or from large water sources at lower depths in an open system). The current application of the revised SoilCover model is to predict moisture redistribution throughout the winter in compacted clay covers over mine waste materials. For this application, it should be reasonable to neglect convective heat transfer.

Sublimation of ice (i.e., direct solid to vapour phase mass transfer) is also neglected in the frozen zone behind the freezing front. Sublimation depends on the partial vapour pressure difference between ice and the surrounding air, but the vapour pressure of ice is not included in the model formulation. If water changes phase to vapour due to a vapour pressure difference between the liquid water and air, then some of the ice must melt to increase the liquid water volume to that predicted by the soil freezing curve. This scenario is included in the model formulation.

The numerical model does not account for heat and mass transfer across a snow layer. However, a proven approach to this problem does not appear in the literature. Snow cover effects are a fundamental problem when modelling winter conditions in the field. Heat and mass transfer through snow layers is difficult to model because the physical and thermal properties of snow crystals are continually changing and this in turn changes the hydraulic and thermal properties of the snow layers. For example, the thermal conductivity of snow has been shown to range from $0.046 \mathrm{~W} / \mathrm{cmK}$ for fresh, light snow, to 
$0.326 \mathrm{~W} / \mathrm{cmK}$ for old, dense snow (Stepphuhn, 1981). If adequate meteorological data including snow depth and density are available, then the temperature of the soil surface beneath the snow pack can be approximated using a simple Fourier heat conduction formulation. Perhaps a simpler estimation of soil surface temperature can be obtained using data reported by Stepphuhn (1981). He reports that the difference in air to soil temperature across a snow layer in Eastern Europe ranged from $1.1{ }^{\circ} \mathrm{C}$ per centimeter of snow when the snow was $0-10 \mathrm{~cm}$ thick, to $0.1^{\circ} \mathrm{C}$ per centimeter of snow when the snow was $70-80 \mathrm{~cm}$ thick.

\subsection{The Laboratory Data Modelling Program}

The Laboratory data modelling program achieved three objectives listed below.

1) The simulation program verified that temperature and moisture content profiles measured by Jame (1980) could be simulated using the proposed theoretical approach.

2) The sensitivity analysis permitted some conclusions to be made about the sensitivity of the computed results to small changes in certain soil property input parameters.

3) The process examined the use of arbitrarily chosen ice impedance factors in soil freezing models.

\subsubsection{General Comments Regarding the Simulated Temperature and Moisture Profiles}

Figures 6.1 to 6.3 compare the computed and simulated temperature and water (liquid and ice) content profiles for the three freezing tests reported by Jame (1980). 

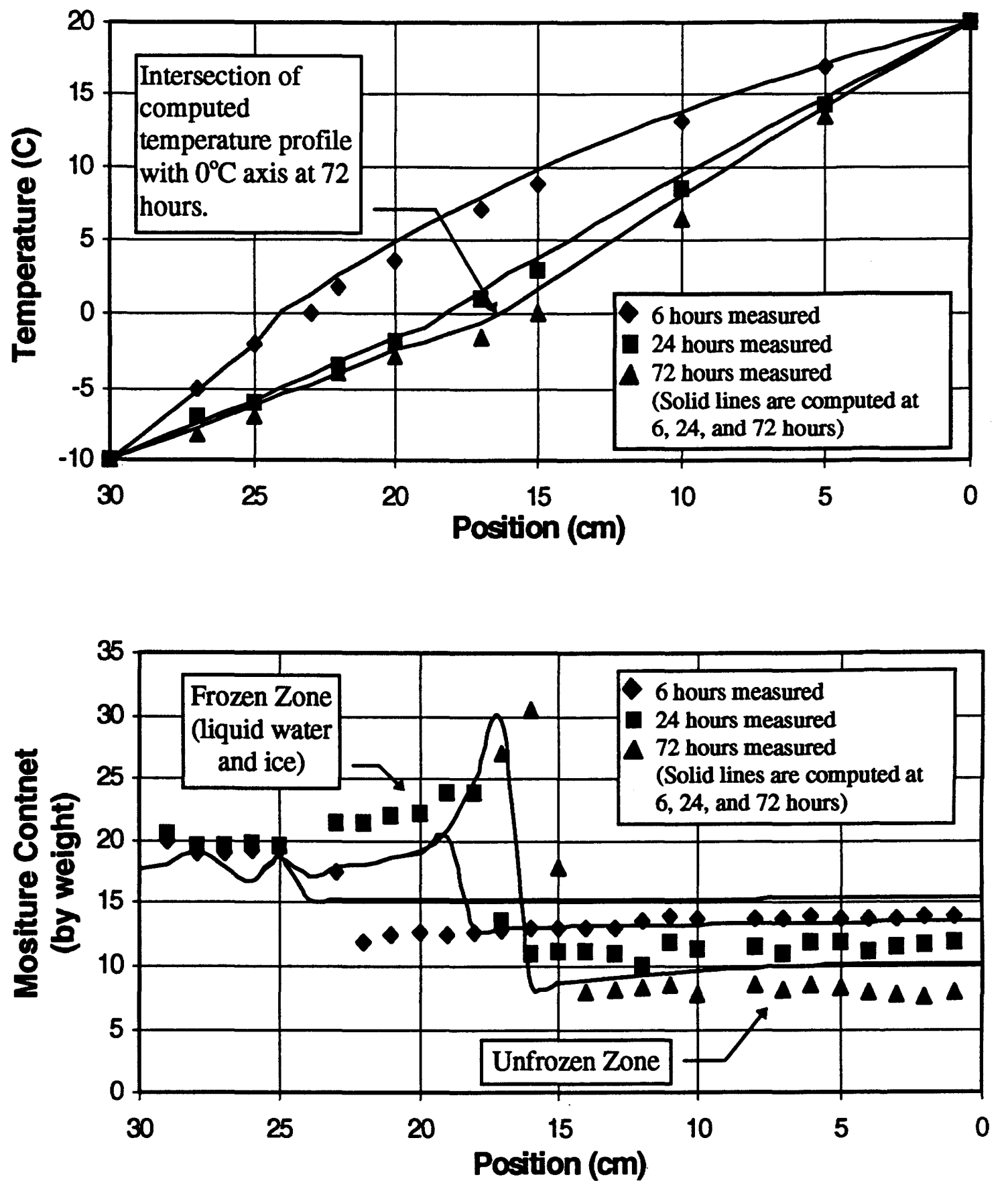

Figure 6.1 Modelling Results of Test 1 

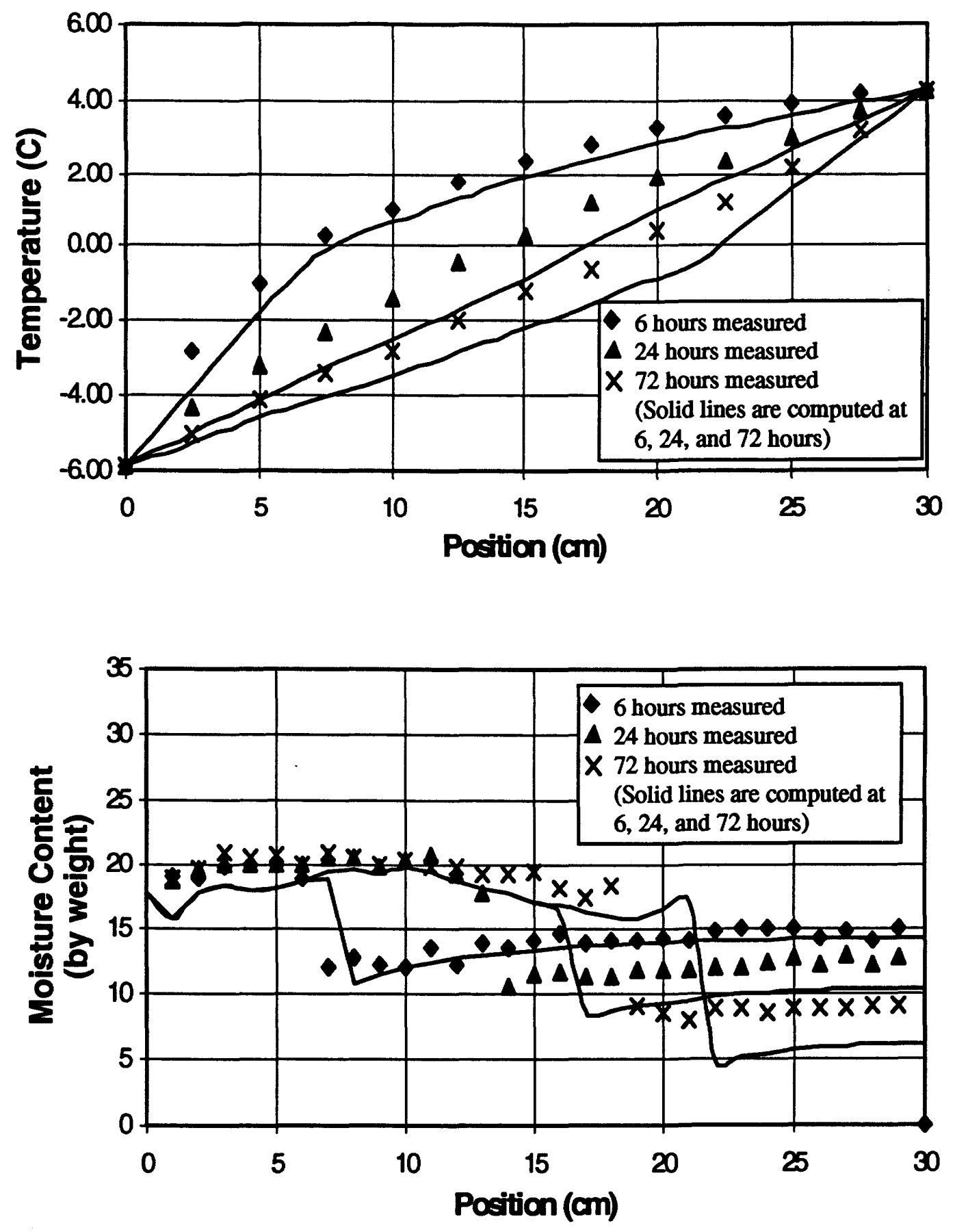

Figure 6.2 Modelling Results of Test 2 

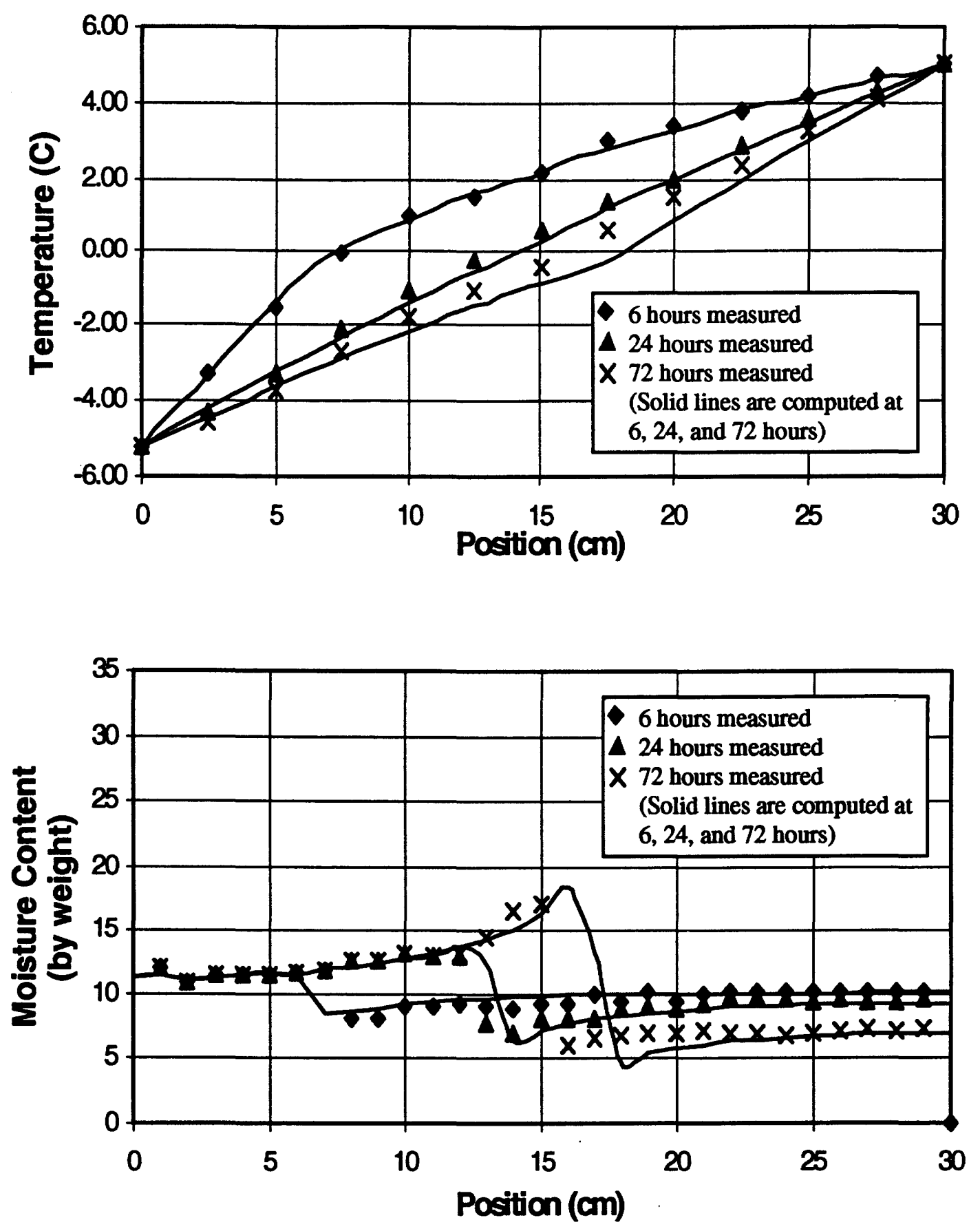

Figure 6.3 Modelling Results of Test 3 
The simulated temperature and total moisture content profiles fit the experimental data with varied accuracy. The agreement between the computed and measured liquid water contents and temperatures seem to vary depending on the initial water content and temperature boundary conditions of the freezing test being simulated. Jame (1977) selected the initial and boundary conditions such that different temperature gradients were imposed on the soil at different initial water contents. He also ensured that the initial water contents were low enough to prevent frost heave from occurring in the silty material during closed system testing.

During freezing test 1 (Figure 6.1) the initial uniform temperature was $20^{\circ} \mathrm{C}$ and the initial water content was $15.6 \%$. The cold end temperature was set at $-10^{\circ} \mathrm{C}$ which induced a thermal gradient of about $1{ }^{\circ} \mathrm{C} / \mathrm{cm}$ throughout the horizontal column. In this simulation the computed temperatures are within 3.3\% of the measured values at both 6 hour and 24 hour times. At 72 hours, the computed temperatures lag behind the measured values to a maximum of $5 \%$ at the frost front. The frost front is assumed to be the intersection of the computed temperature profile with the $0{ }^{\circ} \mathrm{C}$ axis in Figure 6.1.

The computed moisture content values are compared with measured ice and unfrozen water contents in the lower chart of Figure 6.1. In this figure, the agreement between measured and computed values is less accurate. The computed ice contents (i.e., left side of Figure 6.1) are a maximum of $18 \%$ lower than the measured values in the interval between 6 and 24 hours. Between 48 and 72 hours the advancing frost front appears to become stationary and an ice build up occurs. The quantity of computed ice at the frost front is within $3.3 \%$ of measured ice values except the frost front is positioned about $1 \mathrm{~cm}$ short of the measured frost front. The computed unfrozen water contents are $12 \%$ to $25 \%$ higher than the measured water contents at all times in the tests with the maximum difference occurring during the earlier stages of the simulation. Both the temperature and moisture profiles show excellent trend agreement between the measured and computed values. 
The initial uniform temperature for freezing test 2 (i.e., Figure 6.2) was $5^{\circ} \mathrm{C}$ and the initial water content was $15 \%$. The cold end temperature was set to $-4.25^{\circ} \mathrm{C}$ which resulted in a thermal gradient of $1 / 3^{\circ} \mathrm{C} / \mathrm{cm}$ throughout the column. The computed temperature profiles precede the measured profiles at all times during the simulation. At the 6 hour interval the maximum difference between measured and computed temperatures is $8.3 \%$. At 72 hours the computed temperature profile precedes the measured profile up to a maximum of $2 \mathrm{~cm}$ (or $6.6 \%$ ) at the intersection of the temperature line with the $0^{\circ} \mathrm{C}$ axis.

The computed ice content (i.e., left side of lower chart in Figure 6.2) varies from the measured ice content to a maximum of $7.5 \%$ during the simulation. The difference between the computed and measured frost front positions increased during the simulation to a maximum of $2 \mathrm{~cm}$ (or $6.6 \%$ ) at the end of the test. Figure 6.2 shows good agreement (i.e., less than 5\% error) between computed and measured unfrozen water contents earlier in the simulation, but by 72 hours the percent difference between computed unfrozen water contents and measured water contents is about $30 \%$. Again, there is excellent agreement between measured and computed temperature and moisture content trends, even to the extent that both the measured and computed ice content profiles show a small build up of ice at 72 hours when the advancing frost front became somewhat stationary.

The measured results of Test 2 significantly differ from test 1 in one way. Both tests were carried out using a sample with an initial water content of about $15 \%$. However, in test 1 there was a higher thermal gradient across the column (i.e., $1^{\circ} \mathrm{C} / \mathrm{cm}$ for test 1 compared with $1 / 3^{\circ} \mathrm{C} / \mathrm{cm}$ for test 2). The higher thermal gradient in test 1 seemed to cause a large increase in ice content at the frost front, whereas this did not occur in test 2. At the higher thermal gradient the frost front advanced more slowly and allowed moisture to transfer from the unfrozen zone towards the frost front. Common sense would suggest that the frost front would advance faster at higher thermal gradients, however, this was not the case. This was due to the fact that in test 1, the warm end temperature (i.e., 20 
${ }^{\circ} \mathrm{C}$ ) was twice the magnitude as the cold end temperature (i.e., $-10^{\circ} \mathrm{C}$ ). Thus, even though the thermal gradient was higher in test 1 , more heat had to be removed in test 1 and the frost front advanced more slowly.

The results of freezing test 3 are presented in Figure 6.3. In this test the initial uniform temperature was $5^{\circ} \mathrm{C}$ and the initial water content was $10 \%$. The cold end temperature was set to $-5^{\circ} \mathrm{C}$ which resulted in a thermal gradient equal to $1 / 3{ }^{\circ} \mathrm{C} / \mathrm{cm}$. During this simulation the maximum difference between computed and measured temperature values was $8 \%$ which occurred at the duration of the simulation. At the 6 and 24 hour intervals the maximum difference between computed and measured temperatures is $3 \%$. The computed temperature profile preceded the measured temperature profile by about $1 \mathrm{~cm}$ at the 72 hour mark, and as a result, the frost front in the computed moisture profile is also about $1 \mathrm{~cm}$ (or 3.3\%) ahead of the measured frost front at the 72 hour mark. The maximum difference between computed and measured moisture contents is $7.5 \%$ at the 72 hour mark, while the maximum difference at all other times in the simulation is $3 \%$.

During test 3 there appeared to be a small build up of ice at the frost front in the later stages of the test. The thermal gradient imposed on the sample in test 3 was the same as that of test 2 which showed no ice build up. However, in test 3 the initial water content was only $10 \%$ as compared with $15 \%$ for test 2 . The lower permeability associated with the lower water content in test 2 did not permit as much water flux to the frost front in the early stages of the test. As a result, the frost front advanced rapidly until it approached a thermal steady state condition, at which time water slowly made its way to the frost front and accumulated as ice.

\subsubsection{Reasons for the Discrepancy Between Computed and Measured Results}

The differences between computed and measured temperature and moisture content profiles can be attributed to several factors. These factors fall into two categories: numerical solution technique approximations, and soil property function accuracy. 
Numerical factors are discussed next, and the sensitivity of the computed results to various soil property factors is discussed in the following section.

In the SoilCover finite element computer algorithm, the element stiffness and mass storage matrices are developed at every Gauss point in every element, starting at the ground surface (i.e., element \# 1) and proceeding deeper into the soil. The freezing point depression temperature is determined at each Gauss point based on the local liquid water content at the end of the last time step. If the new temperature at that Gauss point is below the freezing point temperature, the modified heat equation is turned on and the mass transfer equation is turned off. If the new temperature is above the freezing point then the element stiffness and mass storage matrices are formulated using the non-freezing coupled heat and mass transfer equations.

By observing computed Gauss point temperatures and suctions during a simulation, it was noticed that the temperature profile would advance rapidly through the soil until the water at a Gauss point location would start to freeze. At that point, the latent heat of phase change released into the system slowed the advancing cold front and ice would build up. The cold front would then start to advance rapidly again until the next Gauss point temperature was low enough for freezing. In this way, the advancing cold front seemed to speed up, then slow down, then speed up etc. The simulated freezing process was not continuous because Gauss points are located a finite distance from each other. The discontinuous nature of the finite element formulation geometry introduces some error in the computed results.

Another problem related to the finite element formulation geometry is that the program is not able to accurately predict suction values just ahead of the advancing frost front. The numerical model uses suction values to estimate the soil properties at the Gauss points between nodes, but it does so without knowledge of the exact location of the frost front in this region. In the finite element formulation, the Gauss point suctions are estimated based on the suctions at the previous and adjacent nodes. This estimation process can result in 
suctions which are too high in the zone immediately ahead of the frost front (i.e., there are high suctions even though the temperature has not lowered to the point when ice forms). Figure 6.4 illustrates the potential problems introduced by erroneous Gauss point property estimations. In this figure, ice is assumed to form at $0^{\circ} \mathrm{C}$. The estimated Gauss point ice content profile shows similar problems as the suction profile for the case where one node has ice build up and the adjacent node does not. In general, the numerical model can not determine the location of the frost front between adjacent nodes.

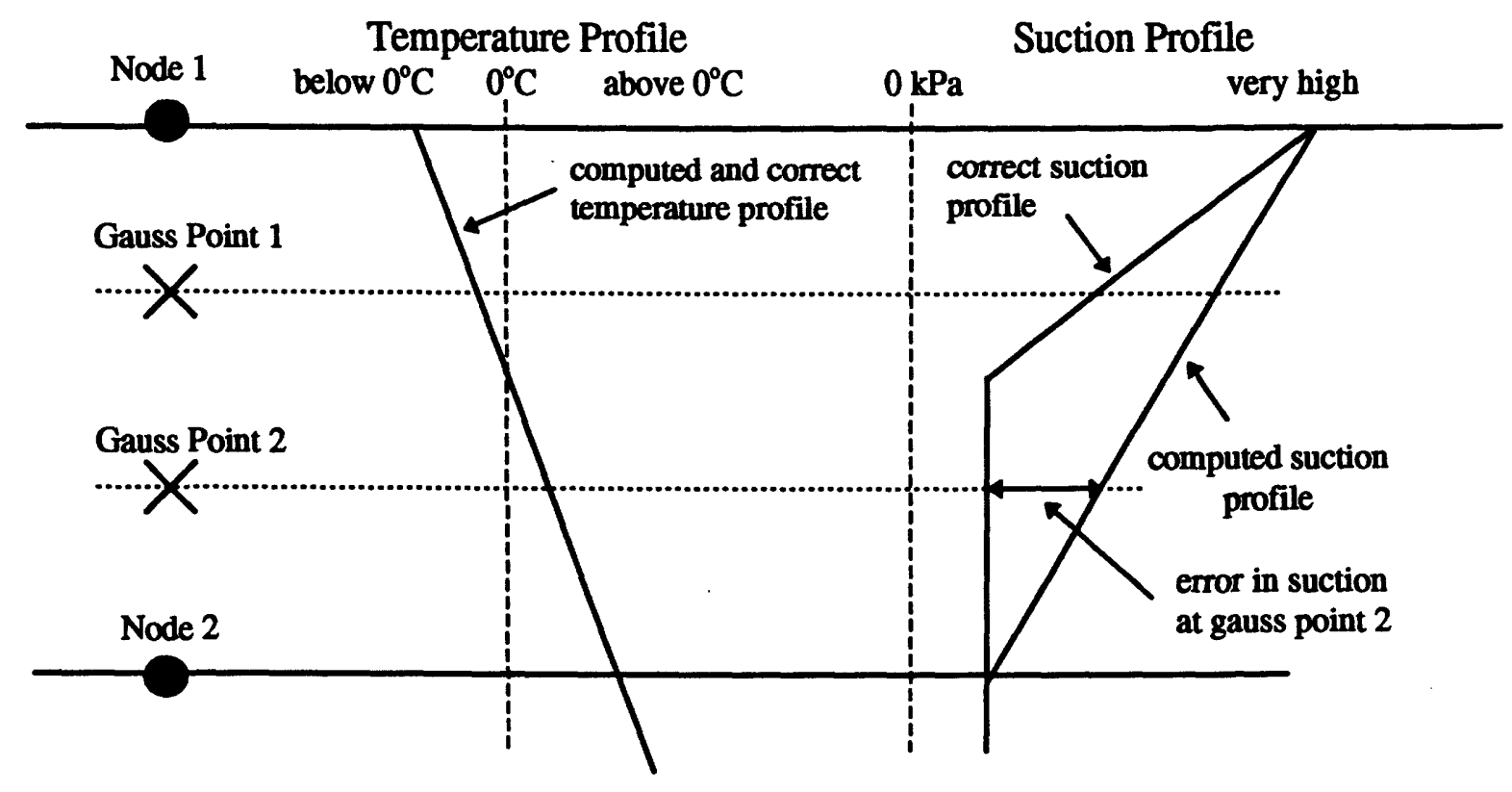

Figure 6.4 Problems With Numerical Gauss Point Suction Estimations

By observing computed Gauss point temperatures and suctions during a simulation it was noticed that the temperature profile through the Gauss points was estimated with good accuracy, but the suction profiles were often erroneous. An overestimate of suctions just ahead of the frost front resulted in a lower than actual estimate of coefficient of permeability. In return, less water flowed to the frost front to change phase and release latent heat. This in turn resulted in a frost front which advanced too rapidly. In other words, less heat was put into the system to slow the frost fronts advance. Clearly, the computed results of test 2 and test 3 (i.e., Figure 6.2 and 6.3) show a frost front slightly 
ahead of the actual frost front. When the frost front advanced too rapidly, too many nodes would change phase and as a result the suctions in the unfrozen zone would tend to get too high. This, in turn, resulted in lower than actual computed water content values in the unfrozen zone.

The finite element method (or any numerical procedure) can only be used to approximate a physical system. The results presented above clearly show that some errors are inherent in the finite element formulation when modelling a rapidly advancing cold front with high moisture redistribution.

\subsection{The Sensitivity of Computed Results to Soil Property Functions}

Other factors accounting for differences between computed and measured results are related to the soil property functions used in the simulations. Figure 6.5 shows three slightly different soil water characteristic curves used in the sensitivity analysis. The curves have the same air entry values and approximately the same values of residual matric suction. However, the three curves have slightly different radii of curvature near their residual water contents. Figures 6.6 to 6.8 show the changes in computed results obtained by making small changes to different soil property functions. Figure 6.6 shows the computed results for the same freezing test simulated with the three soil water characteristic curves.

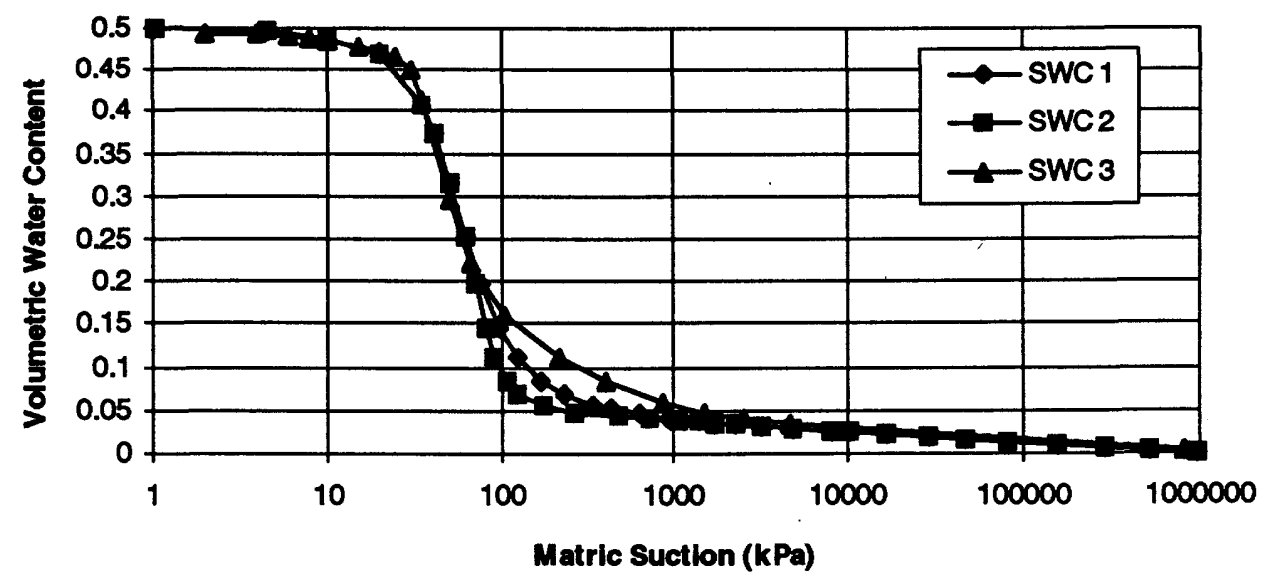

Figure 6.5 Soil Water Characteristic Curves Used in Sensitivity Study 


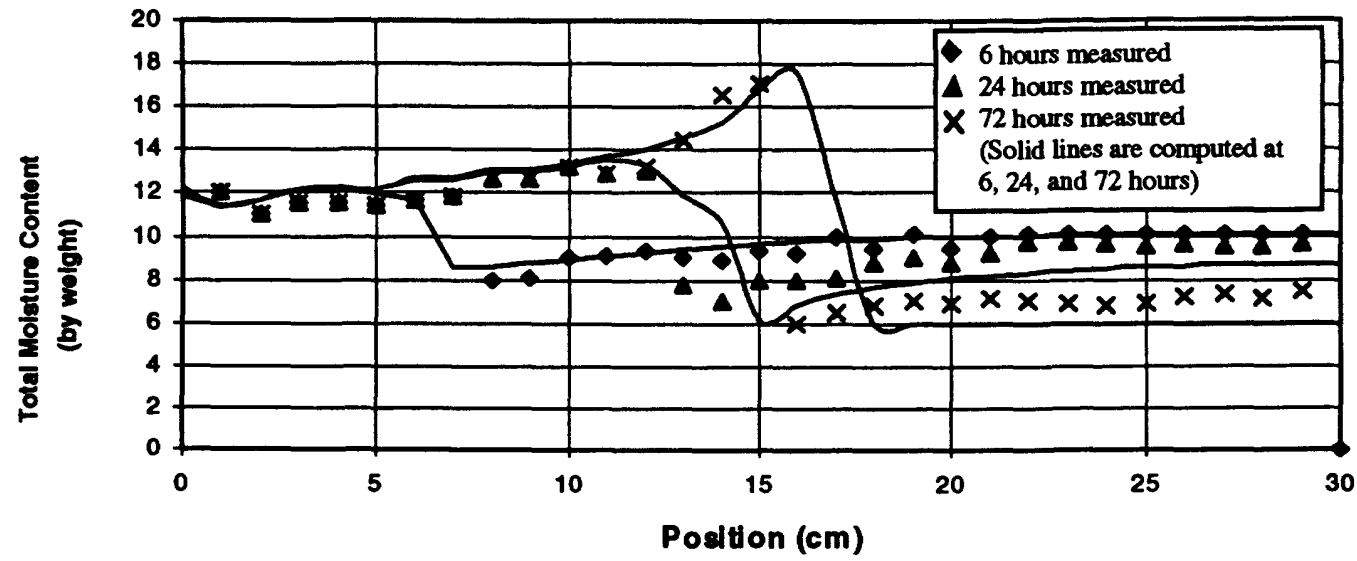

Flgure 6.6a SWC 1

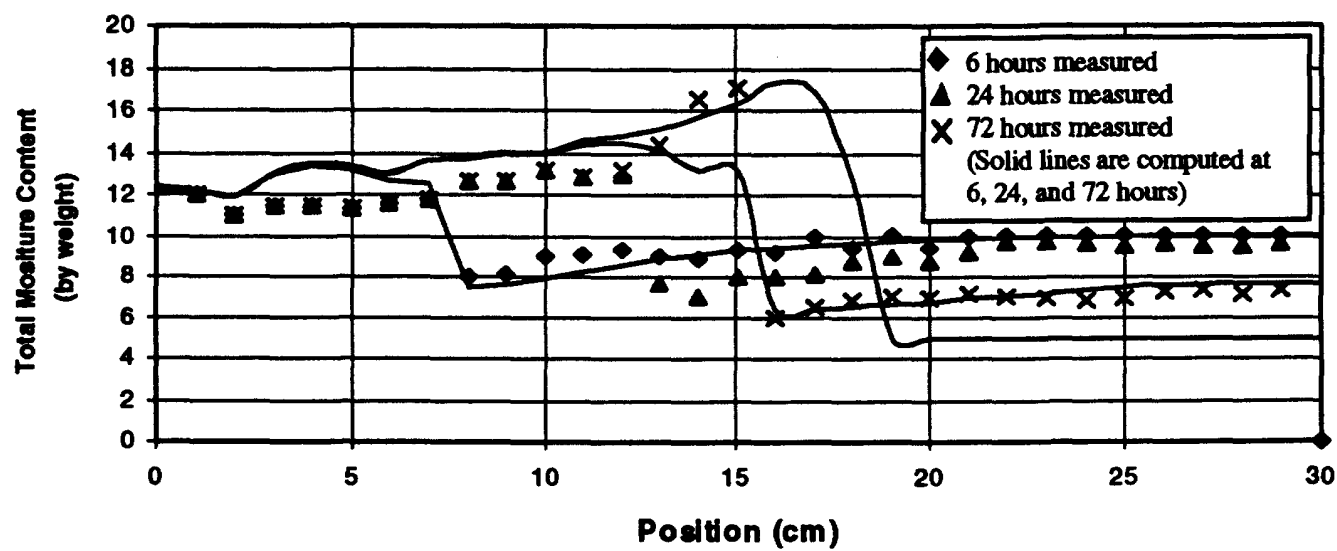

Flgure 6.6b SWC 2

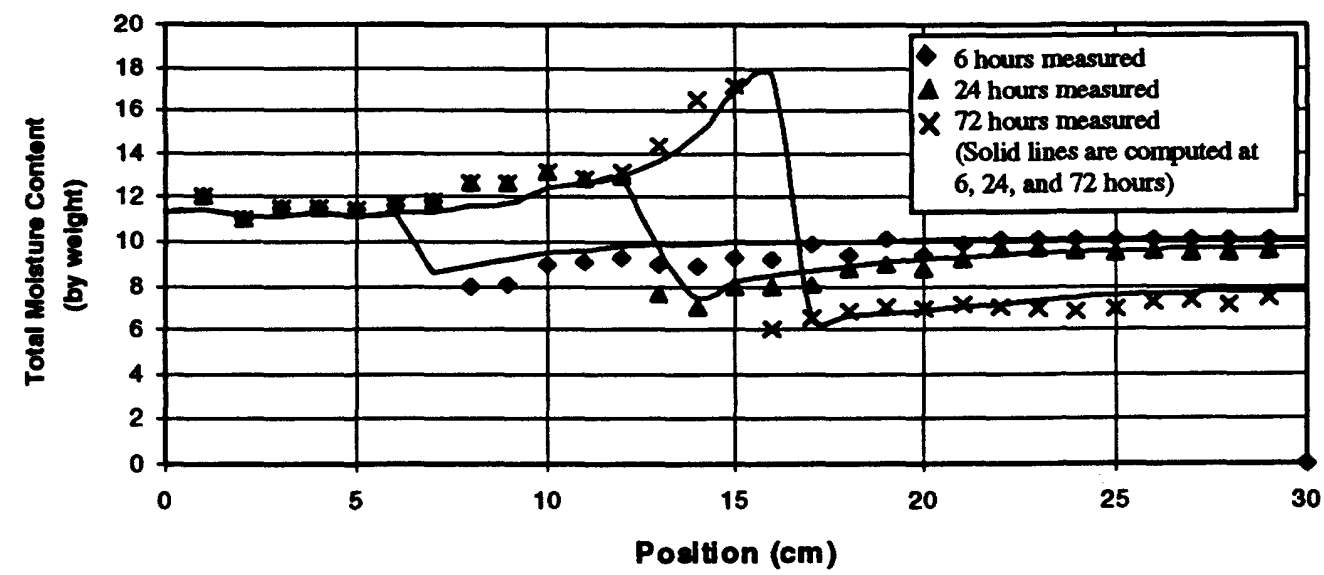

Figure 6.6c SWC 3

Figure 6.6 Moisture Content Profiles Computed Using Three Different Soil Water Characteristic Curves 
The results for the simulations using the soil water characteristic curve with the intermediate radius of curvature (i.e., SWC 1 from Figure 6.5) show a computed frost front which precedes the measured frost front by about $2 \mathrm{~cm}$. In addition, the computed ice contents are slightly high in the initial stages of the simulation, while the computed unfrozen water contents are slightly low in the later stages of the simulation.

The results for the simulations made using the soil water characteristic curve with the largest radius of curvature (i.e., SWC 2) show ice contents even higher than those computed using the soil water characteristic curve marked SWC 1 and unfrozen water contents much lower than those computed with SWC 1 . In addition, the computed frost front precedes the measured frost front by about $3 \mathrm{~cm}$.

The results of the simulations made using the soil water characteristic curve marked as SWC 3 in Figure 6.5 show a computed frost front position which agrees well with the measured frost front position. In addition, ice and unfrozen water contents are in agreement with measured results. In summary, the results presented in Figure 6.6 shows that for this material and test conditions, small errors in the estimation or measurement of the radius of curvature of the soil water characteristic curve near the residual water content had a significant effect on the accuracy of the computed results.

Figure 6.7 compares the simulation results for the same test obtained with slightly different saturated coefficients of permeability. The measured saturated coefficient of permeability for the silica flour used in this study varied about one order of magnitude over a porosity range of 0.48 to 0.51 . Because it was not possible to re-construct the material used by Jame (1980) (i.e., soil type, density), some flexibility was used in the selection of the saturated coefficient of permeability used in this study. 


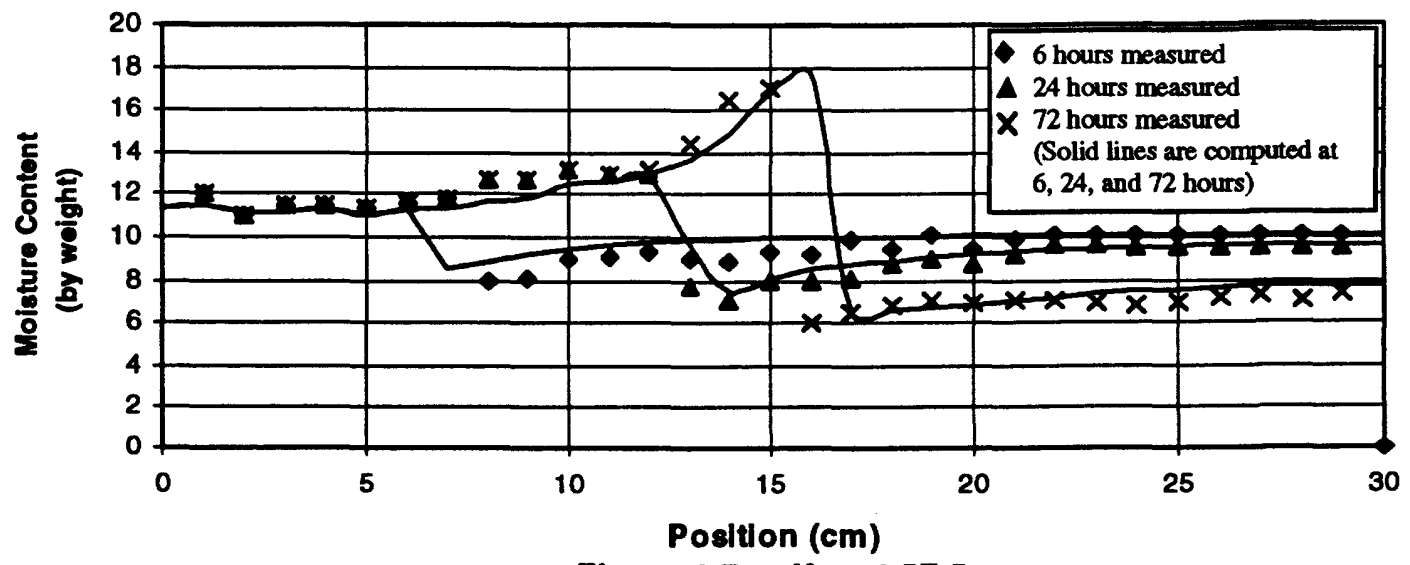

Figure 6.7a $\mathrm{Ks}=4.5 \mathrm{E}-5$

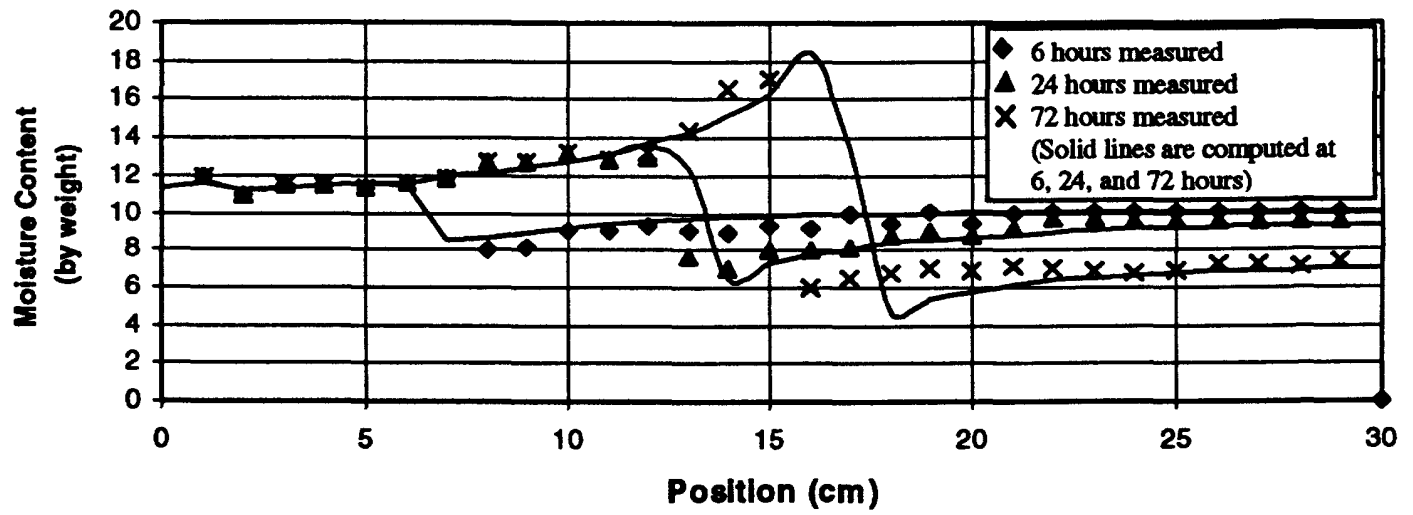

Figure 6.7b $\quad K s=7.0 E-5$

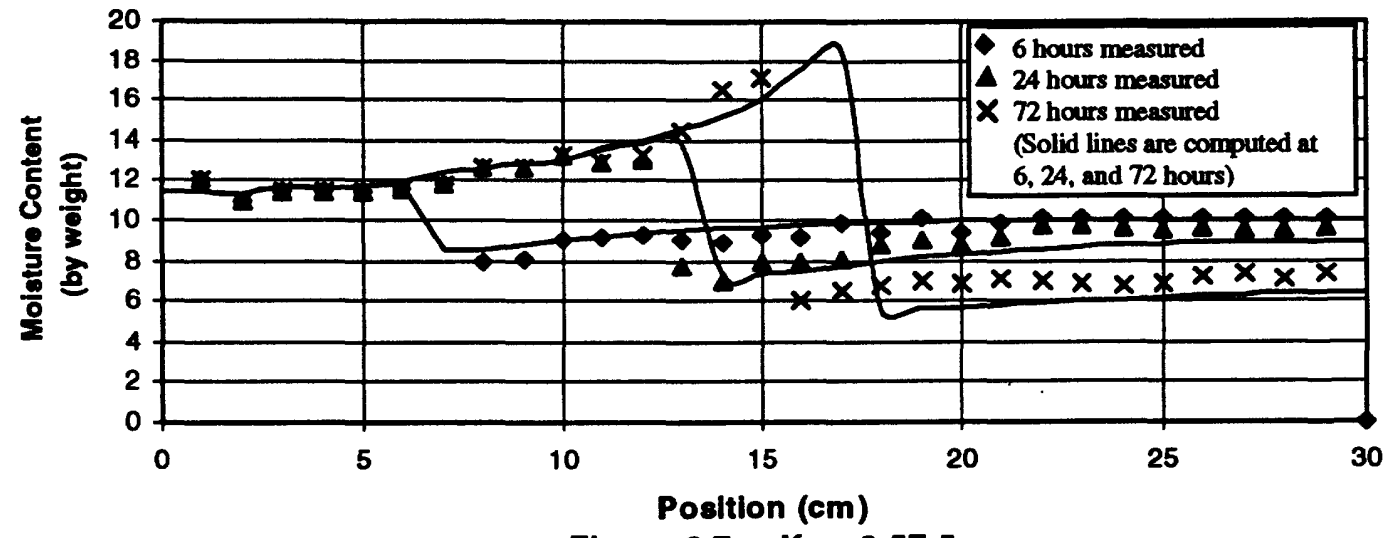

Figure 6.7c $\quad K s=9.5 \mathrm{E}-5$

Figure 6.7 Moisture Content Profiles Computed Using Three Different Saturated Coefficients of Permeability 
Figure 6.7a shows the simulated temperature and moisture contents using a Ksat of $4.5 \mathrm{x}$ $10^{-5} \mathrm{~cm} / \mathrm{s}$. In this figure, there is excellent agreement between computed and measured temperatures and water contents. In Figure $6.7 \mathrm{~b}$ the Ksat is $7.0 \times 10^{-5} \mathrm{~cm} / \mathrm{s}$. It can be noted that the computed frost front slightly precedes the measured frost front, and the computed unfrozen water contents are slightly lower than the measured unfrozen water contents. Finally, with a Ksat of $9.5 \times 10^{-5} \mathrm{~cm} / \mathrm{s}$ (i.e., Figure $6.7 \mathrm{c}$ ) the computed frost front also precedes the measured frost front, and the computed unfrozen water contents are even lower than those computed with the slightly lower Ksat.

Based on the results presented in Figure 6.7 it can be concluded that for this material and test conditions, a higher permeability resulted in a faster moving frost front, which in turn resulted in lower than actual predictions of water content in the unfrozen zones at the end of the test. It can be noted that for all three Ksat values, the agreement between measured and computed unfrozen water contents is better in the earlier stages of the test. This suggests that the Ksat has a greater effect on the rate of frost front advance than on the unfrozen water content. The reason the higher Ksat test shows a lower unfrozen water content at later stages of testing is that more nodes have frozen (due to the faster rate of frost front advance) and higher suctions have advanced deeper into the soil, thus drawing more moisture out of the unfrozen zone.

Figure 6.8 compares the simulated results of the same freezing test using three different ice impedance factor coefficients (i.e., E). In the top chart of the figure, no impedance factor was applied during any stage of the simulation. Contrary to results obtained by Jame (1977), Taylor and Luthin (1978) and others, these results show that reasonable predictions of ice content can be obtained without an arbitrarily chosen ice impedance factor. 


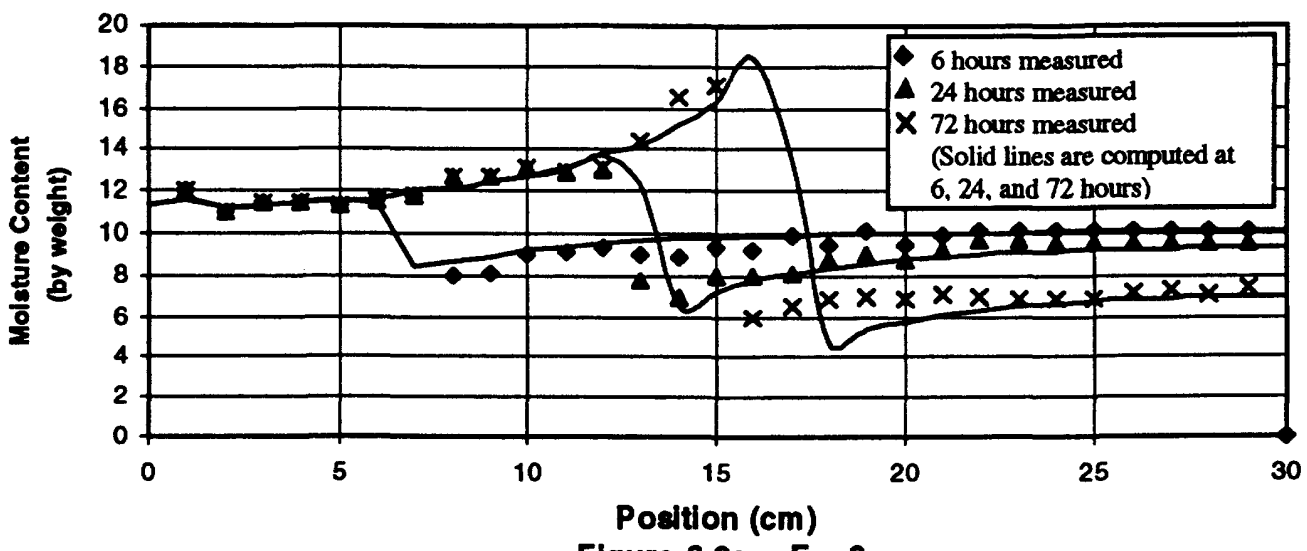

Figure 6.8a $\quad E=0$

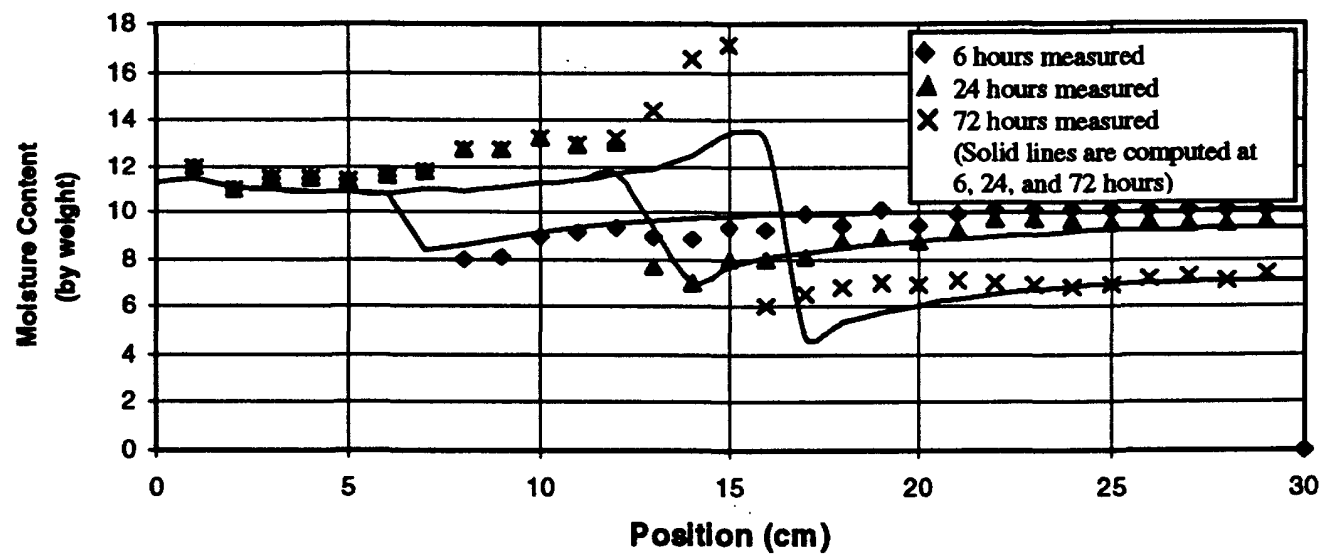

Figure 6.8b $E=6$

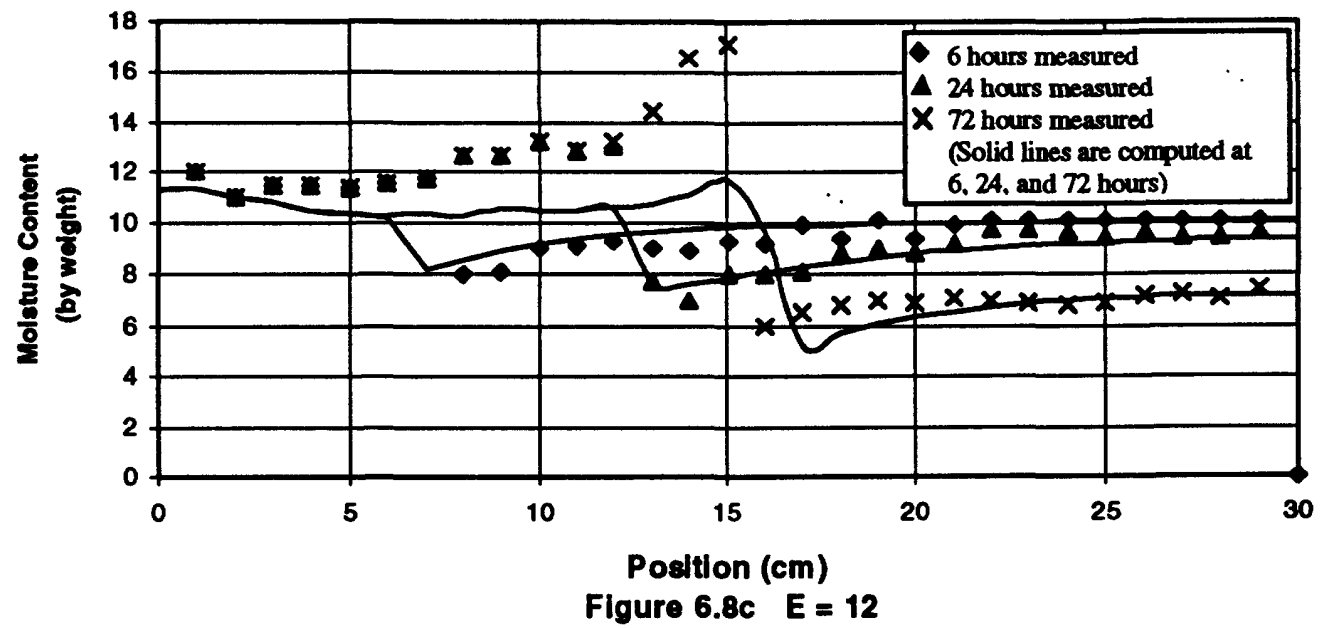

Figure 6.8 Moisture Content Profiles Computed Using Three Different Ice Impedance Factors 
The middle chart of Figure 6.8 shows the computed results obtained with an ice impedance factor coefficient (i.e., E ) of 6 . This implies a 0 to 1.5 order of magnitude drop in permeability as the volumetric ice content increases from 0 to 0.25 . These results clearly show a large under estimation of ice content behind the frost front. The bottom chart shows the computed results obtained using an ice impedance factor coefficient of 12 , or a 0 to 3 order magnitude drop in permeability as the volumetric ice content increases from 0 to 0.25 . This figure shows there is an actual decrease in ice content during the middle stages of testing and then a very slight ice build up as the test approaches the 72 hour mark.

Based on the results presented in Figure 6.8, it can be concluded that application of an ice impedance factor reduces the computed build up of ice behind the frost front. These findings also raise serious questions about the necessity of the ice impedance factor. Harlan (1973) originally hypothesized that the water permeability of a frozen soil could be obtained from the suction - permeability relationship measured at room temperature for any given unfrozen water content. Harlan (1973), Jame (1977) and others were not able to verify this theory. In any case the impedance factor was introduced to make computed results fit measured data. Perhaps the earlier researchers were not able to accurately measure the soil water characteristic curve, or they were not able to extrapolate a reasonable suction - permeability function from the soil water characteristic curve. In this study, the relative permeability function was obtained using the Fredlund et al. (1994) method and it appears to have given reasonable results even in the frozen zone of the soil.

The hypothesis proposed by Harlan (1973) appears logical. If thermodynamic equilibrium requires that the larger pores freeze first (i.e., where the suction is lowest there is less freezing point depression) then any unfrozen water must remain in the smaller pores. In a draining, unfrozen soil, the larger pores also drain first because there is less surface tension across a larger radius of curvature pore. In this case, the remaining water is also stored in the smaller pores. The question can be raised, are these not the same smaller pores which remain unfrozen when the soil freezes? If the suction - permeability 
relationship can predict the permeability of capillary water in the unsaturated pores spaces in a drying soil, then the same relationship should apply to freezing soils. In any case, more work needs to be done to explore these possibilities.

Four tornado plots are presented in Figures 6.9 to 6.12. These plots illustrate the sensitivity of the computed results to the changes in soil property functions discussed above. The first plot compares the position of the computed and simulated temperature profile where it crosses the $0^{\circ} \mathrm{C}$ axis at 6,12 , and 72 hours. The second plot compares the computed and measured ice contents at the freezing front at 6,12 and 72 hours. The third plot compares the unfrozen water contents at the freezing front; and the last plot compares the unfrozen water contents well ahead of the freezing front.

These figures clearly show that slight changes to the soil water characteristic curve or saturated coefficient of permeability cause significant differences between computed and experimental data. The ice impedance factor has a significant effect in the computation of ice contents behind the freezing front and also the computation of unfrozen water content at the freezing front. In general, these figures help illustrate the complex relationships which exist between suctions, temperatures, and liquid flux near the frost front in a freezing soil. 


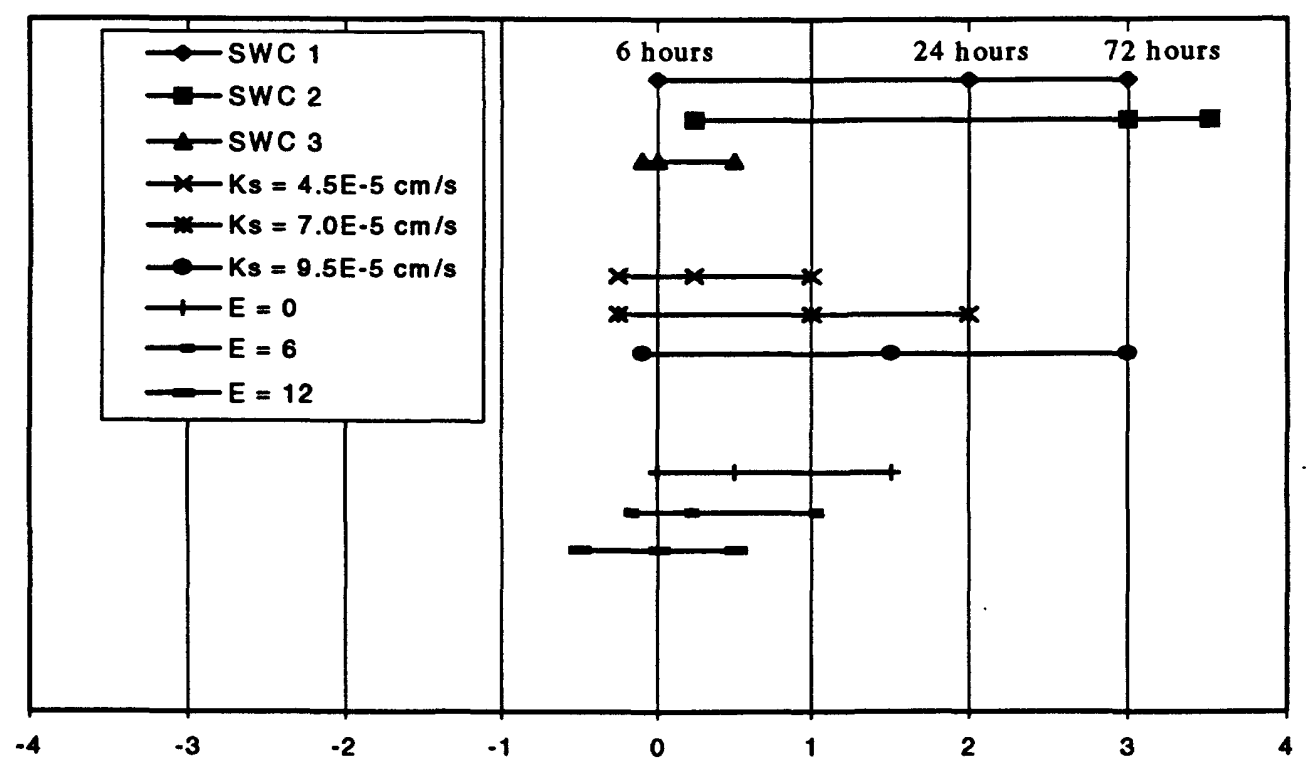

Dlstance Between Computed and Measured Temperature Proflles $(\mathrm{cm})$ at 0 Degree Axls for 6, 24 and 72 Hours

Figure 6.9 Tornado Plot Comparing Position of Temperature Profiles Computed Using Slightly Different Soil Property Functions

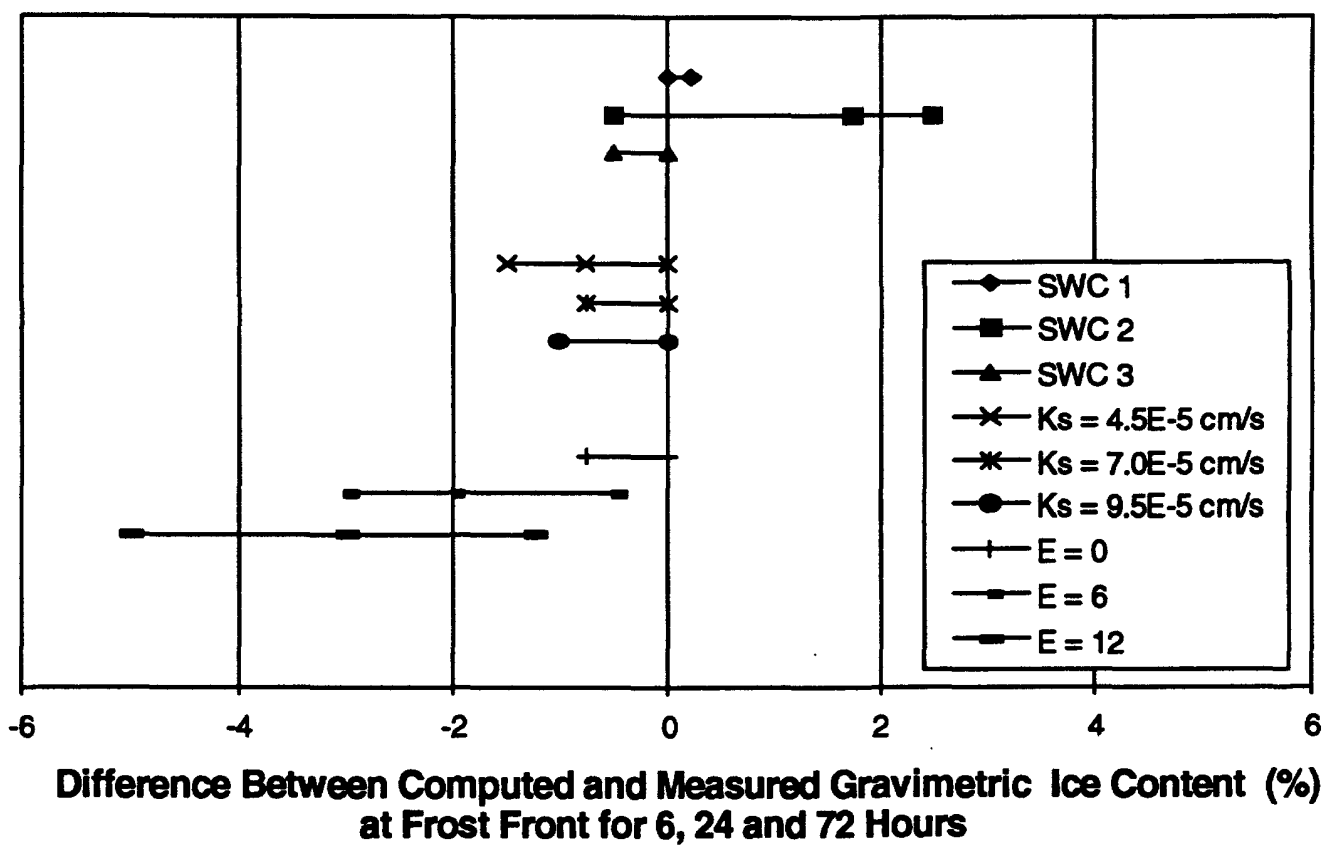

Figure 6.10 Tornado Plot Comparing Ice Contents Computed Using Slightly Different Soil Property Functions 


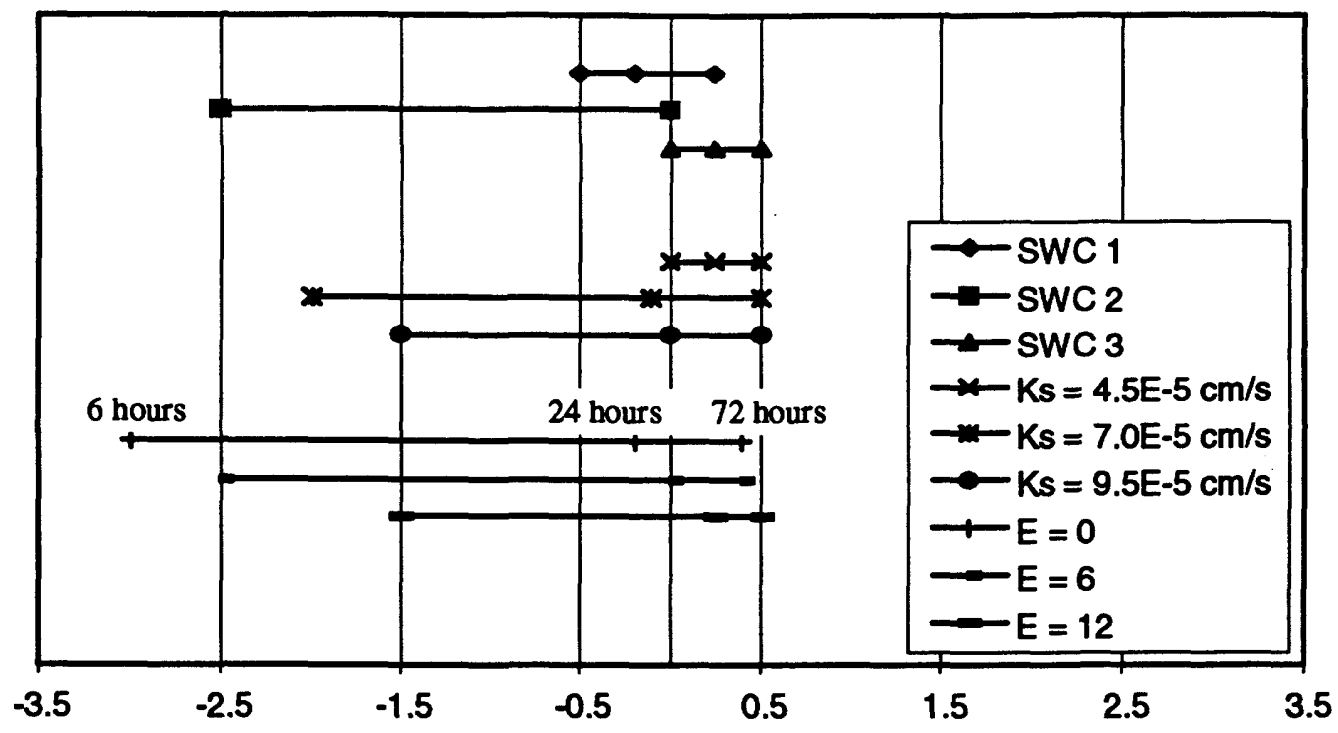

Difference Between Computed and Measured Gravimetric Unfrozen Water Content (\%) at Frost Front for 6, 24, and 72 Hours

Figure 6.11 Tornado Plot Comparing Unfrozen Water Contents Computed Using Slightly Different Soil Property Functions

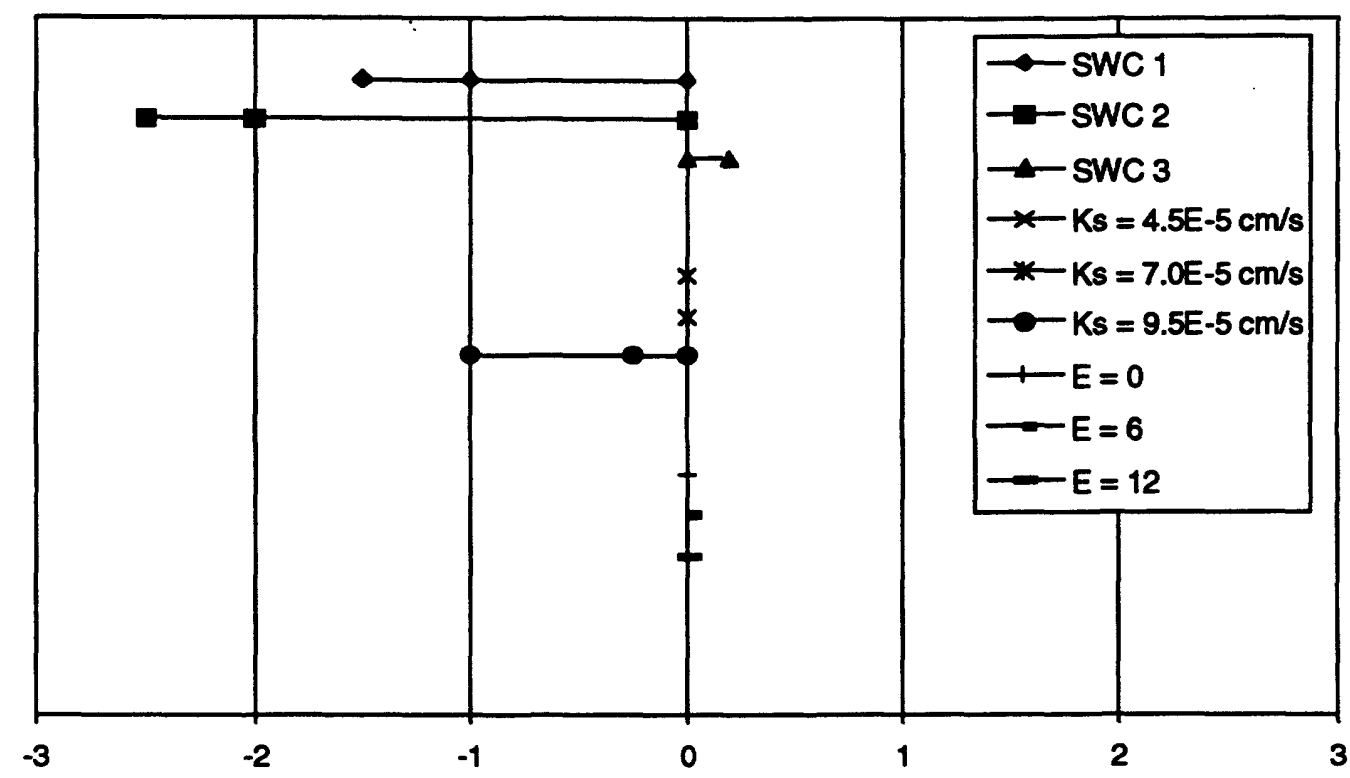

Difference Between Computed and Measured Unfrozen Gravimetric Water Content (\%) Ahead of Freezing Front at 6, 24, and 72 Hours

Figure 6.12 Tornado Plot Comparing Unfrozen Water Contents Computed Using Slightly Different Soil Property Functions 


\section{CHAPTER 7 CONCLUSIONS}

Numerical analysis of freezing and thawing processes in unsaturated soils is complicated by many factors. The desire to obtain a clear understanding of the physics involved in soil freezing has led researchers to develop numerous theoretical models for analysing the problem. The literature shows that many different models have been developed, and that none of them are capable of being a general tool for geotechnical engineers. In fact, most of them were developed as research tools designed to fulfill a unique objective. All of the models, including the modified SoilCover (MEND, 1993) program, are restricted by the assumptions that were made as the models were developed.

Thermodynamic equilibrium theory has been applied to soil freezing with limited success. If the water in a soil is assumed to be held totally by either capillary or adsorptive forces, then it is possible to relate matric suction to sub-zero temperature using the appropriate form of the Clapeyron equation. However, the majority of soils contain both capillary and adsorptive water; thus, the Clapeyron equation falls short. Thermodynamic equilibrium theory, among others, has been used to develop freezing point depression relationships. Jame (1972) showed that the freezing point depression function was the same as the unfrozen water content versus sub-zero temperature function. This is advantageous in numerical modelling because the same curve can be used to determine when the pore-water will begin to freeze (based on unfrozen water contents in the pores) 
and also what the unfrozen water content will be after the majority of pore-water has frozen.

To date, the soil water characteristic curve has been used sparingly in soil freezing analysis. This curve plays a significant role in the modified SoilCover (MEND, 1993) model because it couples the thermal and stress states of the soil in the frozen or partially frozen zones. The unfrozen water content is common to both the soil freezing curve and the soil water characteristic curve which enables the matric suction to be computed for any sub-zero temperature in the soil. When the slope of the soil freezing curve is divided by the slope of the soil water characteristic curve the resulting value is a ratio of proportionality between changing suctions and changing temperatures. It provides the constant values used in the Clapeyron equation, and it is not limited by soil type. This relationship enabled a single equation to be derived which accounted for both the heat flow and mass transfer continuity.

The soil water characteristic curve can be used to compute the relationship between water permeability and suction in unfrozen soils. However, researchers are divided on how to determine the coefficient of permeability in a frozen or partially frozen soil. This division gave rise to the term 'ice impedance factor', an empirical relationship used to calibrate soil freezing models. It was initially intended that an ice impedance factor be used in this program. However, results of initial testing showed that the permeability versus suction function that was derived from the soil water characteristic curve using equations recently presented by Fredlund et al. (1994) gave excellent results for liquid flux in the frozen and unfrozen zones. This is a very important finding and needs to be investigated in more detail.

The objective of this research program was to present theory for heat and mass transfer in freezing unsaturated soils. The theory was then verified using laboratory data. The laboratory modelling program was carried out by simulating soil freezing of fine silica flour with large water fluxes in the unfrozen zone. The results of these simulations 
verified that the freezing analysis capabilities of the revised numerical model were working. A sensitivity analysis was also done to compare computed results using slightly different soil property functions. The results of the sensitivity study clearly showed that small changes to the soil water characteristic curve or saturated coefficient of permeability value caused significant differences between computed and experimental results. Inclusion of an ice impedance factor had a significant effect in the computation of ice contents behind the freezing front, and also in the computation of unfrozen water content at the freezing front. The results of this study suggest that an ice impedance factor is not necessary if an accurate permeability versus suction relationship can be predicted.

The field modelling exercise (presented in Appendix A) was carried out to demonstrate that the revised model was capable of both freezing and thawing analysis, and that it could do so within the framework of the existing computer code. In the field data simulations, good agreement between computed and measured temperature profiles was obtained for times when ice was not present in the soil. An incorrect calculation of pore ice affected the calculated thermal conductivity values which in turn resulted in a calculated frost front that advanced too deep into the soil during one period of the simulation. The accuracy of the computed results could be improved by using a more accurate soil freezing curve relationship, as it directly affects the computed ice content values. In addition, more information is needed about the bottom and top boundary conditions (i.e., in the waste rock and beneath the snow pack).

This revised numerical model should be considered as a first step in developing a truly year round soil heat and mass transfer model. The current formulation uses suction and temperature as dependent variables and relies heavily on the soil freezing and soil water characteristic curves. More information about the complex relationship between these curves is needed. In particular, it would be desirable to be able to accurately predict the soil freezing curve from soil water characteristic curve data. More research should also be carried out to explore the relationships between soil water characteristic curves and suction - permeability functions in freezing soils. 
Future modifications to the model should include: adding convective heat transfer and sublimation effects; adding an algorithm to couple the soil surface with the snow and the snow surface with the atmosphere; and incorporating unsaturated soil mechanics theory to account for total stress, effective stress, ice pressures and frost heave.

The revised numerical model is useful to engineers in its current form. If a user has a clear understanding of the limitations and advantages inherent in the model, then he or she should be able to carry out field response and predictive modelling on a year round basis. 


\section{REFERENCES}

Anderson, Duwayne M. and N.R. Morgenstern, 1973. Physics, Chemistry, and Mechanics of Frozen Ground: A Review. Proceedings: Second International Conference on Permafrost. Yakutsk, USSR., pp. 257-288.

Beskow, G., 1935. Soil Freezing and Frost Heaving with Special Application to Roads and Railroads. The Swedish Geological Society, C, no. 375. Year Book no. 3 (Translated by J.O. Osterberg), Technological Institute, Northwestern University.

Black, Patrick B. and Allen R. Tice, 1989. Comparison of Soil Freezing Curve and Soil Water Curve Data for Windsor Sandy Loam. Water Resources Res., vol. 25(10), pp. 2205-2210.

Black, Partick B. and Mark J. Hardenberg, Editors, 1991. Historical Perspective in Frost Heave Research, The Early Works of S. Taber and G. Beskow. CRREL Special Report 91-23. U.S. Army Corps of Engineers, Hanover, New Hampshire.

Boyoucous, G.J., 1920. Degree of Temperature to Which Soils Can Be Cooled Without Freezing. Journal of Agricultural Research., vol. 20, pp. 267-269.

Dakshanamurthy, V. and D.G. Fredlund, 1981. A Mathematical Model for Predicting Moisture Flow in an Unsaturated Soil under Hydraulic and Temperature Gradients. Water Resources Research Journal., vol. 17(3), pp. 714-722.

De Vries', D.A., 1952. The Thermal Conductivity of Soils. Mededelingen Van Der Landbouwhogeschool te Wageningen, 52, pp. 1-73.

Dirksen, C. and R.D. Miller, 1966. Closed-System Freezing of Unsaturated Soil. Soil Science Society of America Proceedings., vol. 30, pp. 168-173. 
Everett, D.H., 1961. The Thermodynamics of Frost Damage to Porous Solids. Faraday Soc. Trans. 57 (465, Part 9), pp. 1542-1551.

Farouki, Omar, 1981. Thermal Properties of Soils. CRREL Monograph 81-1. U.S. Army Corps of Engineers, Hanover, New Hampshire, USA.

Fisher, R.A., 1924. The Freezing of Water in Capillary Systems: A Critical Discussion. Journal of Physical Chemistry., vol. 28, pp. 36-67.

Flerchinger, Gerald Norma, 1987. Simultaneous Heat and Water Model of a SnowResidue-Soil System. Ph.D. Thesis, Washington State University, Pullman, Washington.

Fredlund, D.G. and H. Rahardjo, 1993. Soil Mechanics for Unsaturated Soils. John Wiley \& Sons, Inc. New York.

Fredlund, D.G., Xing, Anqing, and Shangyan Huang, 1994. Predicting the Permeability Function for Unsaturated Soils Using the Soil-Water Characteristic Curve. Canadian Geotechnical Journal., vol. 31, pp. 533-546.

Fredlund, D.G., and Anqing Xing, 1994. Equations for the Soil-Water Characteristic Curve. Canadian Geotechnical Journal, vol. 31, pp. 521-532.

Gilpin, R.R., 1980. A Model for the Prediction of Ice Lensing and Frost Heave in Soils. Water Resources Research, Vol. 16 (5). pp. 918-930.

Gosnik, J.P., Kawasaki, K., Osterkamp, T.E., and J. Holty, 1988. Heat and Moisture Transport During Annual Freezing and Thawing. Proceedings: Fourth International Conference on Permafrost. July 17-22. Fairbanks, Alaska., pp. 355360.

Gray, D.M., R.J. Granger, and G.E. Dyck, 1985. Overwinter Soil Moisture Changes. Transactions, ASCE., vol. 28(2), pp. 442-447.

Guymon, Gary L, and James N. Luthin, 1974. A Coupled Heat and Moisture Transport Model for Arctic Soils. Water Resources Res., vol. 10(5), pp. 995-1001.

Harlan, R.L., 1973. Analysis of Coupled Heat-Fluid Transport in Partially Frozen Soil. Water Resource Res., vol. 9, pp. 1314 - 1323.

Hoekstra, P., 1966. Moisture Movement in Soil Under Temperature Gradients with the Cold Side Temperature Below Freezing. Water Resource Res., vol. 2, pp. 241250. 
Hromadka, Ted, 1987. A Nodal Domain Integration Model of Two-Dimensional Heat and Soil-Water Flow Coupled by Soil-Water Phase Change. CRREL Special Report 87-9. U.S. Army Corps of Engineers, Hanover, New Hampshire.

Jame, Y.W., 1972. Temperature Effects on Phase Composition of a Partially Frozen Soil. M.Sc. Thesis. University of Saskatchewan, Saskatoon, Canada.

Jame, Y.W., 1977. Heat and Mass Transfer in Freezing Soil. Ph.D. Thesis. University of Saskatchewan, Saskatoon, Canada.

Jame, Y.W. and D.I. Norum, 1980. Heat and Mass Transfer in Freezing Unsaturated Porous Media. Water Resource Res., vol. 16, pp. 811-819.

Johansen, O., 1975. Thermal Conductivity of Soils, Ph.D. Theses. Trondheim, Norway. (CRREL Draft Translation 637, 1977).

Joshi, Bhaskar, 1993. A Finite Element Model for the Coupled Flow of Moisture and Heat is Soils under Atmospheric Forcing. M.Sc. Dissertation, University of Saskatchewan, Saskatoon, Canada.

Jumikis, A.R., 1966. Thermal Soil Mechanics. Rutgers University Press., New Brunswick, N.J.

Kemper, W.D., 1960. Water and Ion Movement in Thin Films as Influenced by the Electrostatic Charge and Diffuse Layer of Cations Associated with Clay Mineral Surfaces. Soil Science Society of America Proceedings., vol. 24, pp. 10-16.

Kersten, M.S., 1949. Laboratory Research for the Determination of the Thermal Properties of Soils. ACFEL Technical Report 23. AD 712516.

Konrad, Jean-Marie, and Norbert R. Morgenstern, 1980. A Mechanistic Theory of Ice Lens Formation in Fine-Grained Soils. Canadian Geotechnical Journal., vol. 17, pp. 473-486.

Lange, G.R. and H.L. McKim, 1966. Saturation, Phase Composition, and Freezing-Point Depression in a Rigid Soil Model. Permafrost: Proceedings of an International Conference. National Academy of Sciences, Washington, D.C., pp. 187-192.

Low, P.F., D.M. Anderson and P. Hoekstra, 1968. Some Thermodynamic Relationships for Soil At or Below the Freezing Point: 1. Freezing Point Depression and Heat Capacity. Water Resources Res., vol. 4, pp. 379-394.

Lunardini, V.J., 1991. Heat Transfer with Freezing and Thawing. Elsevier Science Publishers B.V., New York, N.Y. 
Mageau, D.W. and N.R. Morgenstern, 1980. Observations on Moisture Migration in Frozen Soils. Canadian Geotechnical Journal., vol. 17, pp. 54-60.

Martynov, G.A., 1959. Heat and Moisture Transfer in Freezing and Thawing Soils. In Principles of Geocryology. Nathional Research Council of Canada, Technical Translation 1065, Chapter VI.

Maulem, Y. 1976. A New Model for Predicting the Hydrualic Conductivity of Unsaturated Porous Media. Water Resource Research., vol. 12, pp. 513-522.

MEND, 1993. SoilCover, User's Manual for an Evaporative Flux Model. University of Saskatchewan, Saskatoon, Saskatchewan, Canada.

McGaw, R.W., R.L. Berg, and J.W. Ingersoll, 1982. An Investigation of Transient Processes in an Advancing Zone of Freezing. Proceedings: Fourth International Conference on Permafrost. July 17-22. Fairbanks, Alaska., pp. 821-825.

Miller, Robert D., 1966. Phase Equilibria and Soil Freezing. Proceedings of an International Conference. National Academy of Sciences, Washington, D.C., pp. 193-197.

Miller, Robert D., 1973. Soil Freezing in Relation to Pore Water Pressure and Temperature. Proceedings: Second International Conference on Permafrost. Yakutsk, USSR., pp. 244 - 352.

Nakano, Y., A.R. Tice, J.L. Oliphant, and T.F. Jenkins, 1982. Soil-Water Diffusivity of Unsaturated Soils at Sub-Zero Temperatures. Proceedings: Fourth International Conference on Permafrost. July 17-22. Fairbanks, Alaska., pp. 889-893.

Nersesova, Z.A., and N.A. Tsytovich, 1966. Unfrozen Water in Frozen Soils. Permafrost: Proceedings of an International Conference. National Academy of Sciences, Washington, D.C., pp. 230-233

Nixon, J.F., 1975. The Role of Convective Heat Transport in the Thawing of Frozen Soils. Canadian Geotechnical Joumal., vol. 12, pp. 425-429.

Nixon, J.F., 1991. Discrete Ice Lens Theory for Frost Heave in Soils. Canadian Geotechnical Journal., vol. 28, pp 843-859.

Nixon, J.F., 1992. Discrete Ice Lens Theory for Frost Heave Beneath Pipelines. Canadian Geotechnical Journal., vol. 29, pp. 487-497.

O'kane, M, 1995. Instrumentation and Monitoring of Engineered Soil Covers for Acid Generating Mine Waste. M.Sc. Thesis, Department of Civil Engineering, University of Saskatchewan, Saskatoon, Saskatchewan, Canada. 
Oliphant, J.L., A.R. Tice and Y. Nakano, 1982. Water Migration Due to a Temperature Gradient in Frozen Soil. Proceedings: Fourth International Conference on Permafrost. July 17-22. Fairbanks, Alaska., pp. 951-956.

Penner, E., 1959. The Mechanism of Frost Heaving in Soils. Highway Research Board. Bulletin 225. Washington. pp. 1-22.

Penner, E., 1967. Heaving Pressure in Soils During Unidirectional Freezing. Canadian Geotechnical Journal., vol. 4, pp. 398-408.

Philip, J.R. and D.A. de Vries, 1957. Moisture Movement in Porours Materials under Temperature Gradients. Transaction, American Geophysical Union., vol 38, no. 2, pp. 222-232.

Schofield, R.K., 1935. The PF of the Water in Soil. Transactions, 3rd. International Congress of Soil Science., vol. 2, pp. 37-48.

Smith, W.O., 1939. Thermal Conductivitites in Moist Soils. Soil Science Society of America Proceedings., vol. 4, pp. 521-524.

Smith, M.W., 1985. "Models of Soil Freezing", in Field and Theory, Lectures in Geocryology., M. Church and O. Slaymaker editors. University of British Columbia Press.

Swanson, D.A., 1995. Predictive Modelling of Moisture Movement in Engineered Soil Covers for Acid Generating Mine Waste. M.Sc. Thesis, Department of Civil Engineering, University of Saskatchewan, Saskatoon, Saskatchewan, Canada.

Taber, S., 1929. The Mecanichs of Frost Heaving. Journal of Geology., vol. 38, pp. 308317.

Takagi, Shunsuke, 1966. Theory of Freezing-Point Depression with Special Reference to Soil Water. Proceedings of an International Conference. National Academy of Sciences, Washington, D.C., pp. 216-224.

Tao, Y.-X. and D.M. Gray, 1994. Prediction of Snowmelt Infiltration into Frozen Soils. Numerical Heat Transfer, Part A., vol. 26, pp. 643-665.

Taylor, George S. and James N. Luthin, 1978. A Model for Coupled Heat and Moisture Transfer During Soil Freezing. Canadian Geotechnical Journal., vol. 15, pp. 548555. 
Tice, A.R., D.M. Anderson, and A.Banin, 1976. The Prediction of Unfrozen Water Contents in Frozen Soils from Liquid Limit Determinations. CRREL Report 76-8, Hanover, New Jersey.

Williams, P.J., 1964. Unfrozen Water Content of Frozen Soils and Moisture Suction. Geotechnique., vol. 14, pp. 133-142.

Williams, P.J., 1966. Suction and Its Effects in Unfrozen Water of Frozen Soils. Proceedings of an International Conference. National Academy of Sciences, Washington, D.C., pp. 225 - 229.

Williams, P.J., 1967. Properties and Behaviour of Freezing Soils. Norwegian Geotechnical Institute., Oslo. Publication 72.

Wilson, G. Ward, 1990. Soil Evaporative Fluxes for Geotechnical Engineering Problems. Ph.D. Thesis. University of Saskatchewan, Saskatoon, Saskatchewan, Canada.

Yong, Raymond, 1965. Soil Suction Effects on Partial Soil Freezing. Highway Research Rec., vol. 68, pp. 31-42. 


\section{APPENDIX A FIELD DATA MODELLING APPLICATION}

\section{A1 Introduction to Field Data Modelling}

To illustrate a practical use for the revised model, a simulation was carried out to compare computed and measured over winter soil temperature data that was collected from an instrumented soil cover over mine waste at Equity Mine near Houston, B.C. The field data modelling program also confirmed that all of the revisions to the SoilCover (MEND, 1993) program were compatible with the existing program. In particular, it was necessary to ensure that a smooth transition between non-freezing and freezing conditions took place as the surface boundary conditions changed and the upper layers of soil began to freeze. Also, it was necessary to ensure that the transition between a freezing soil and a thawing soil did not disrupt the solution process. Recall that the laboratory data modelling program did not deal with thawing soils.

The soil cover system at Equity Mine is comprised of $50 \mathrm{~cm}$ of compacted glacial till overlain by $30 \mathrm{~cm}$ of loose glacial till. For modelling purposes, $1 \mathrm{~m}$ of waste rock was assumed to exist below the compacted till. The loose till cover at the field site was vegetated to decrease erosion and to reduce precipitation and snow melt runoff. Reduced runoff may lead to higher infiltration which in turn keeps the cover system near saturation. However, vegetation increases evapotranspiration which tends to reduce saturation. It is 
desirable to keep the cover saturated because this reduces the infiltration of oxygen which reacts with water in the waste rock. This results in the oxidation of sulfide bearing minerals in the waste rock and leads to acid mine drainage problems.

A detailed description of the instrumentation installed at the Equity site is given by O'kane (1995). The instrumentation consists of a fully automated weather station which measures air temperature, relative humidity, wind speed, precipitation (excluding snow fall), and global and net radiation. Vertical culverts were installed at three locations on the cover to give access to thermal conductivity sensors which were inserted horizontally into the cover and waste rock at different depths. The thermal conductivity sensors were connected to automatic data loggers to record suctions and temperatures at different depths. Neutron probe access tubes, lysimeters, and oxygen probes were also installed at various locations on the cover. The data used in this study was obtained from a culvert stationed on the south west face of the main dump.

\section{A2 Soil Properties Used in Field Data Modelling Program}

The types of soil properties required as input for SoilCover are the same as those discussed in section 4.3. Details of the laboratory testing and field calibration of these soil properties are given by Swanson (1995). All the soil property relationships presented below are the same as those used by Swanson (1995) for the non-freezing modelling of the soil cover at Equity Silver Mine with the exception of the soil freezing curves for the three materials used in this study. Modifications to the soil properties for frozen soils are included in the program computer code.

Figure A1 shows soil water characteristic curves used by Swanson (1995) in modelling the

Equity cover system. The waste rock soil water characteristic curve used in the study is based on the curve for Beaver Creek sand. It fits the trend expected for waste rock and it indicates that a capillary break should exist between the compacted clay and the coarse waste rock. 
Figure A2 shows the relative coefficients of permeability for the three soils used in this study. The saturated coefficient of permeability of the loose till, compacted till, and waste rock were $5.7 \times 10^{-7} \mathrm{~cm} / \mathrm{s}, 2.0 \times 10^{-8} \mathrm{~cm} / \mathrm{s}$, and $1.3 .0 \times 10^{-3} \mathrm{~cm} / \mathrm{s}$ respectively. The specific gravity of the till was calculated to be 2.77 , with a field dry density of $1.74 \mathrm{Mg} / \mathrm{m}^{3}$ and a calculated porosity of 0.37 . The average dry density of the compacted till was estimated to be $1.85 \mathrm{Mg} / \mathrm{m}^{3}$ with a porosity of 0.33 (Swanson, 1995). The porosity of the Beaver Creek sand was calculated to be 0.4 with an air entry value of $3.8 \mathrm{kPa}$ (Wilson, 1990).

The soil freezing curves for the three materials are shown in Figure A3. These curves were computed using the soil water characteristic data curves shown in Figure A1 and the form of the Clapeyron equation presented by Black and Tice (1989) (i.e., discussed in Chapter 2). In applying the Clapeyron equation, it is necessary to know whether the pore-water is held by capillary or adsorptive forces. In this case, the water in the waste rock was assumed to be held by capillary forces and water in the tills was assumed to be held totally by adsorptive forces.

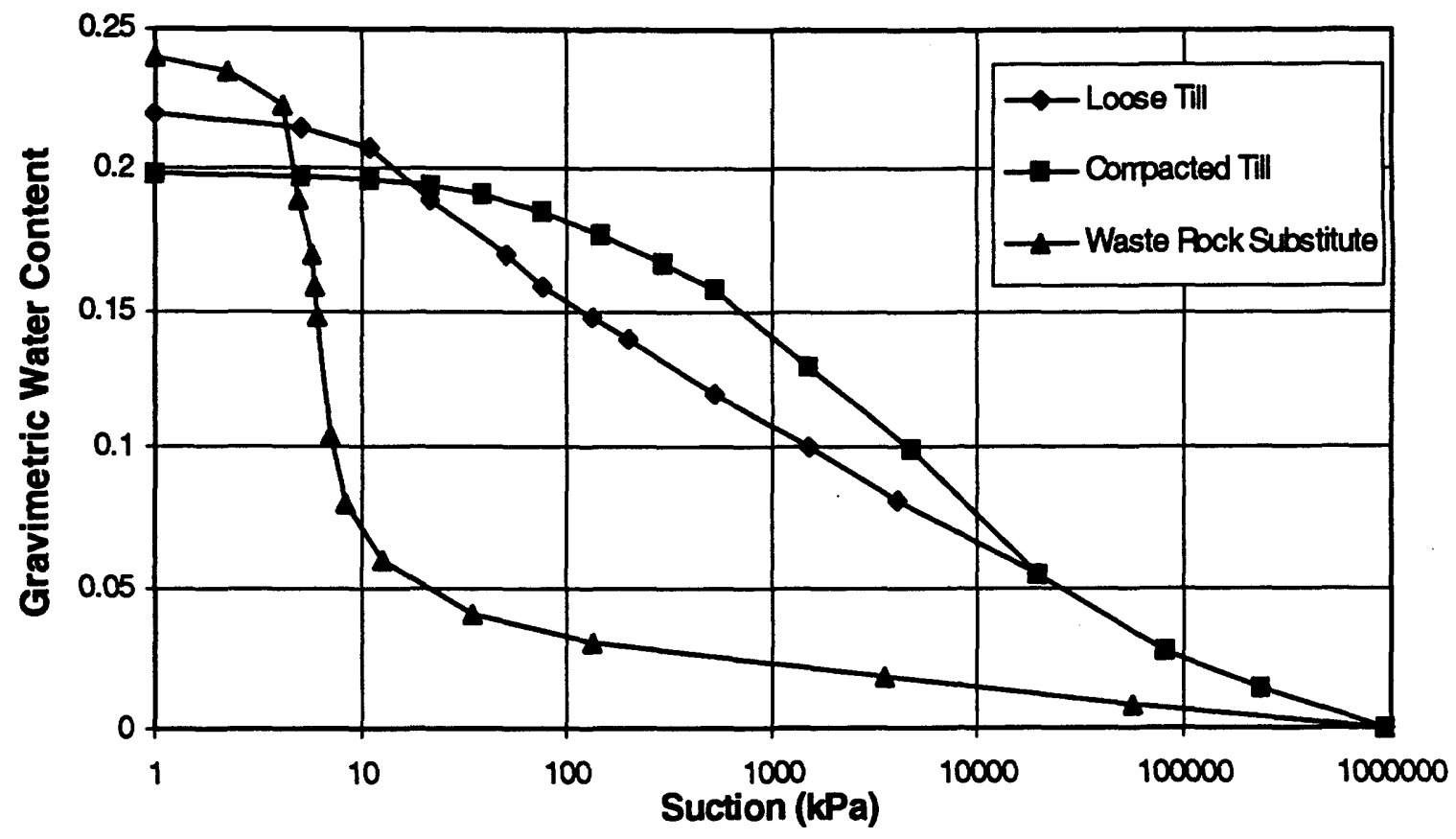

Figure A1 Soil Water Characteristic Curves for Modelling Field Data (after Swanson, 1995) 


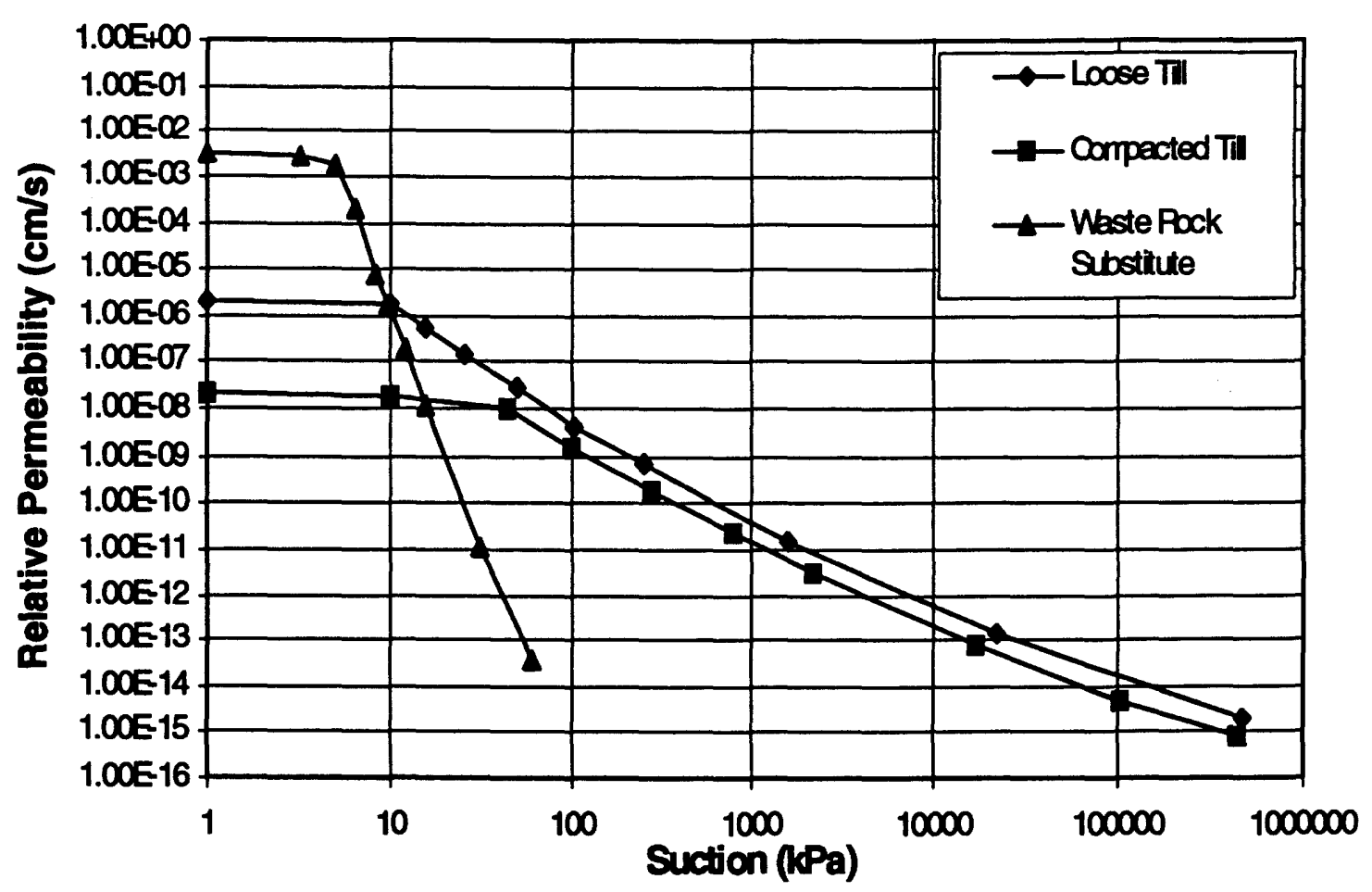

Figure A2 Relative Coefficients of Permeability for Modelling Field Data (modified after Swanson, 1995)

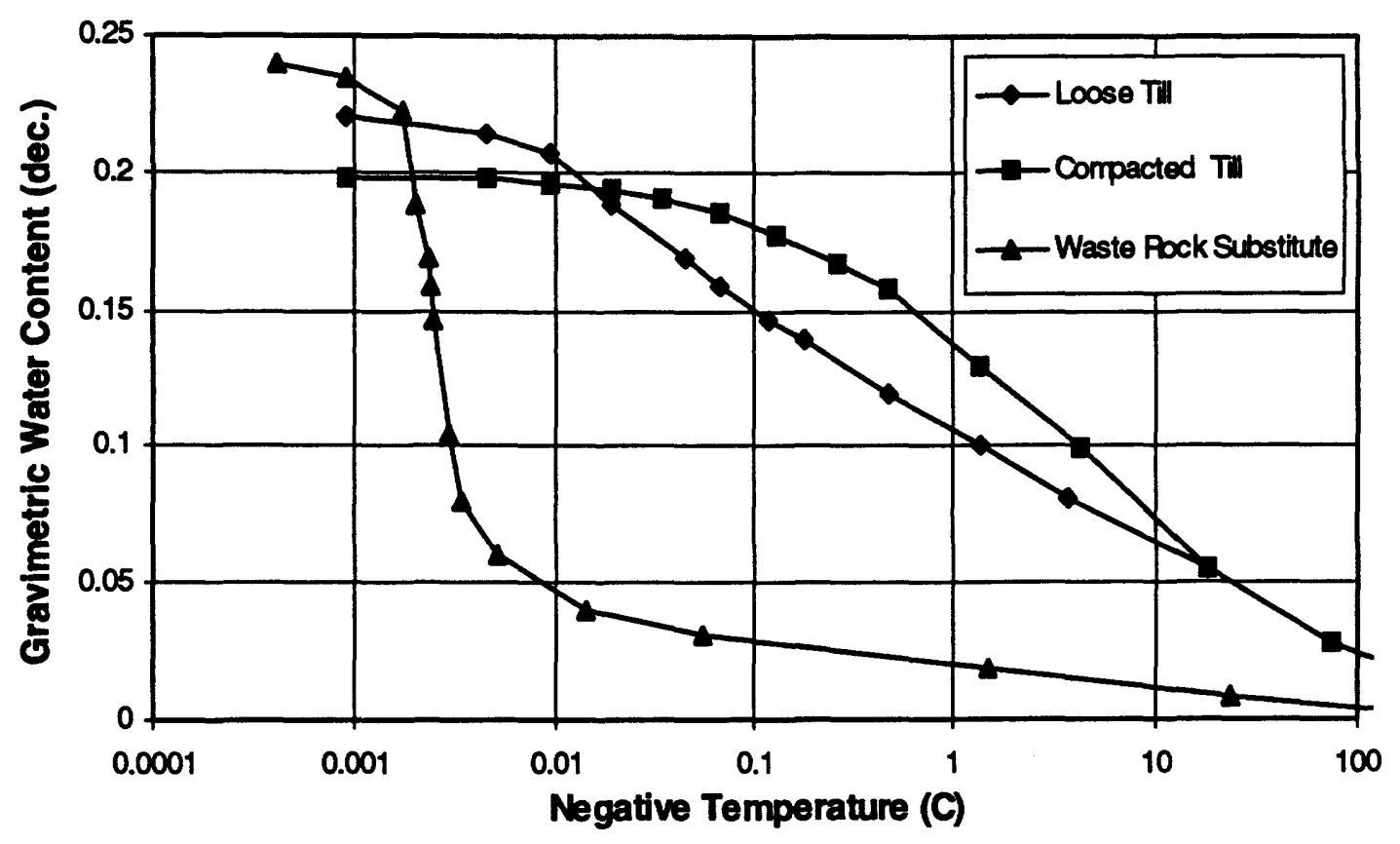

Figure A3 Soil Freezing Curve Data Used in Field Modelling Program 
Figure A4 shows the linearized ' $G$ ' function which relates changes in temperature with changes in suction. Recall that this term is required to render the modified heat transfer equation determinate. It should also be noted in Figure A4 that the ' $G$ ' function is constant for all water contents. This is a result of deriving the soil freezing curve from the soil water characteristic curve (i.e., the values of ' $G$ ' in Figure A4 are the constants of proportionality inherent in the Clapeyron equations given by Black and Tice (1989).

Figure A5 shows the thermal conductivity function of each soil for the non-freezing case. The appropriate form of the Johansen (1975) method for computing unfrozen thermal conductivity was matched to the curves in Figure A5 so that the necessary constants could be obtained for use in the method proposed by Johansen (1975) for frozen soils (i.e., Chapter 2). The dry thermal conductivity, $\lambda_{\text {dry }}$, was calculated to be $0.4 \mathrm{~W} / \mathrm{m}^{\circ} \mathrm{C}$ for the both the till and waste rock. The effective solids thermal conductivity, $\lambda_{s}$, was calculated to be $5.05 \mathrm{~W} / \mathrm{m}^{\circ} \mathrm{C}$ and $4.05 \mathrm{~W} / \mathrm{m}^{\circ} \mathrm{C}$ for the till and waste rock respectively.

Figure A6 shows the computed volumetric specific heat of the till and waste rock materials for non-freezing conditions. The volumetric specific heat of a freezing soil is a function of both water and ice contents and is not a single valued function. For the freezing case, the volumetric specific heat is computed by adding the appropriate specific heat for the volume of ice present as described in Chapter 2. This is done within the SoilCover program. 


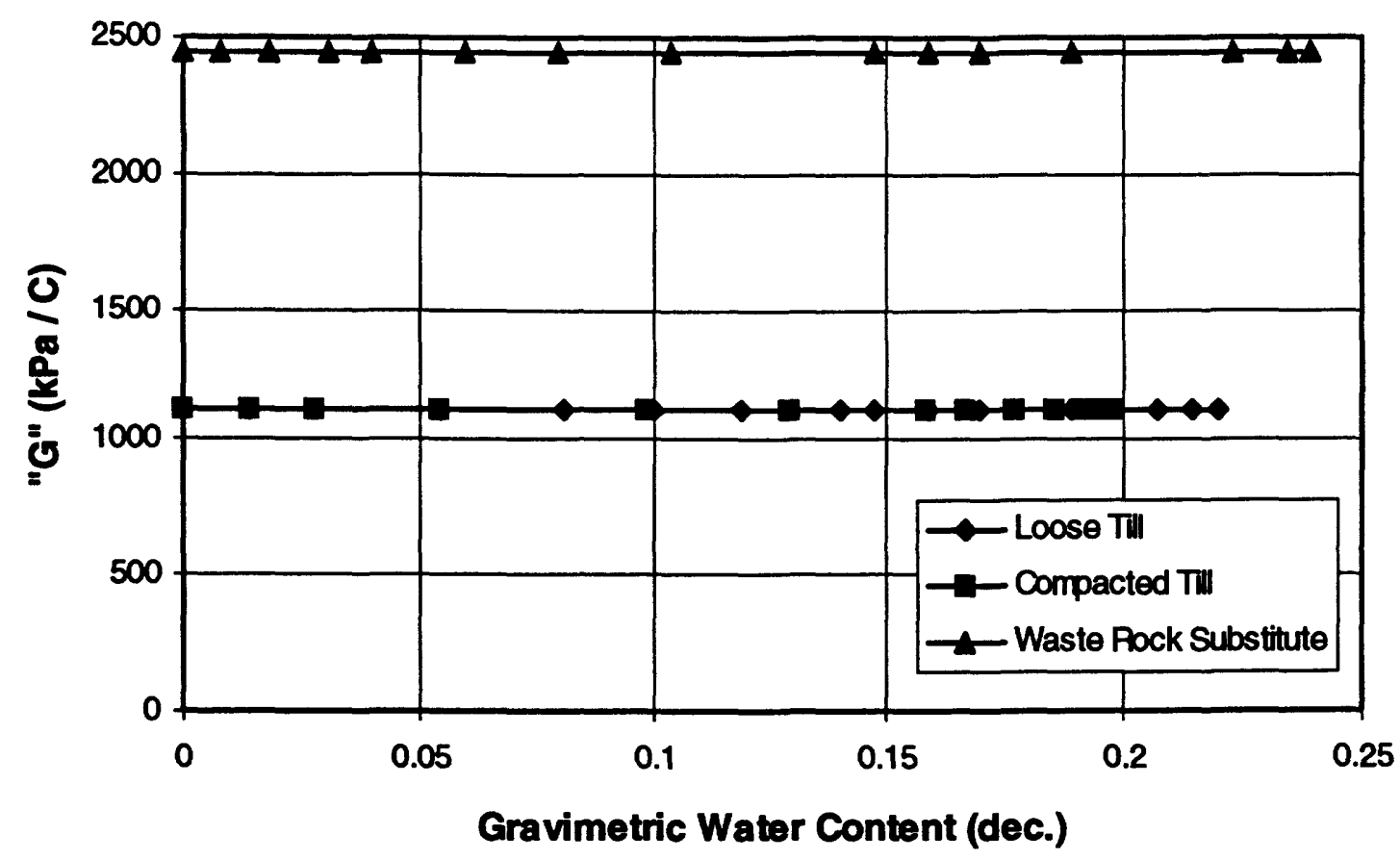

Figure A4 Ratio of Proportionality, 'G' Between Change in Suction and Change in Temperature

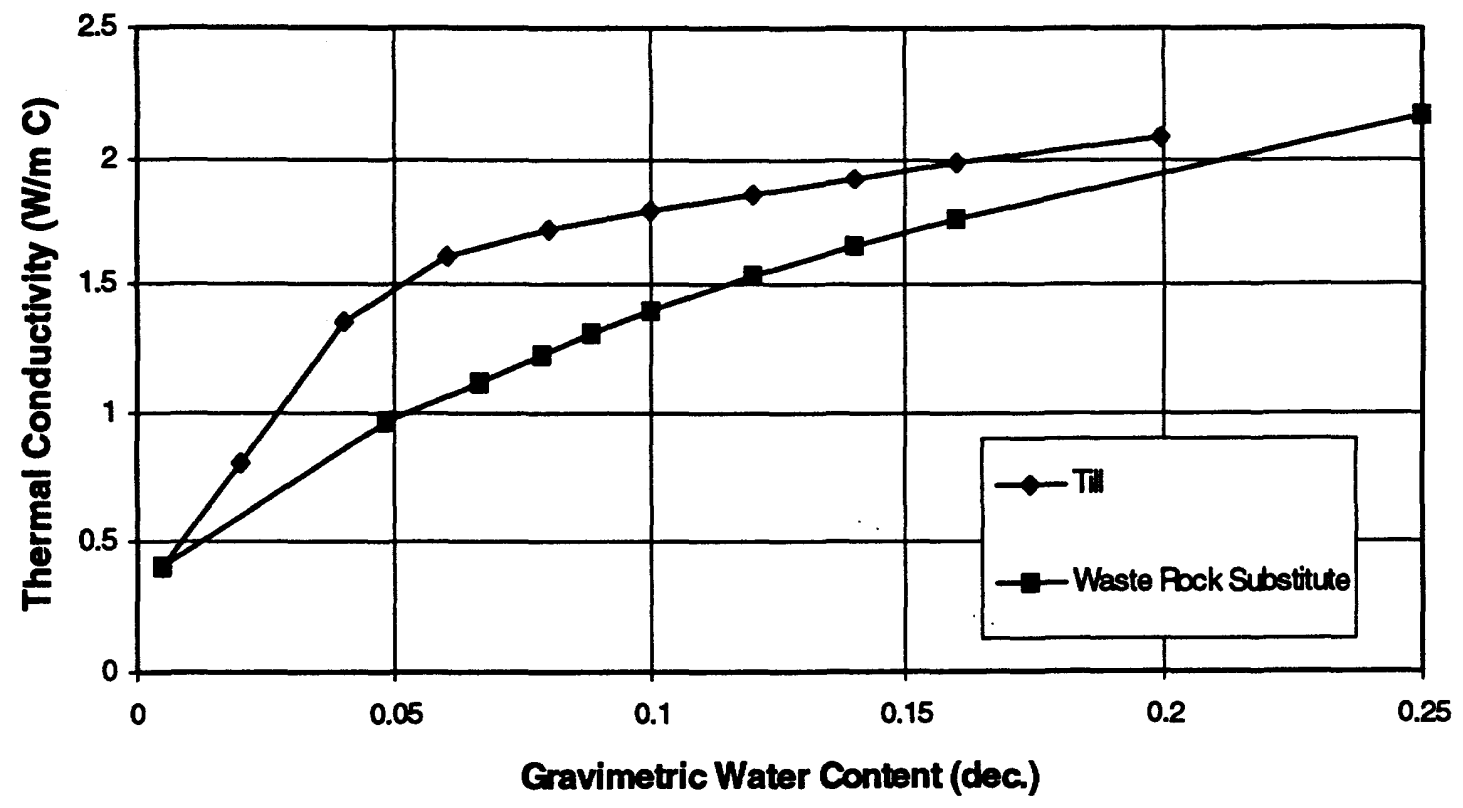

Figure A5 Thermal Conductivity Functions of Cover Materials for Non-Freezing Case (after Swanson, 1995) 


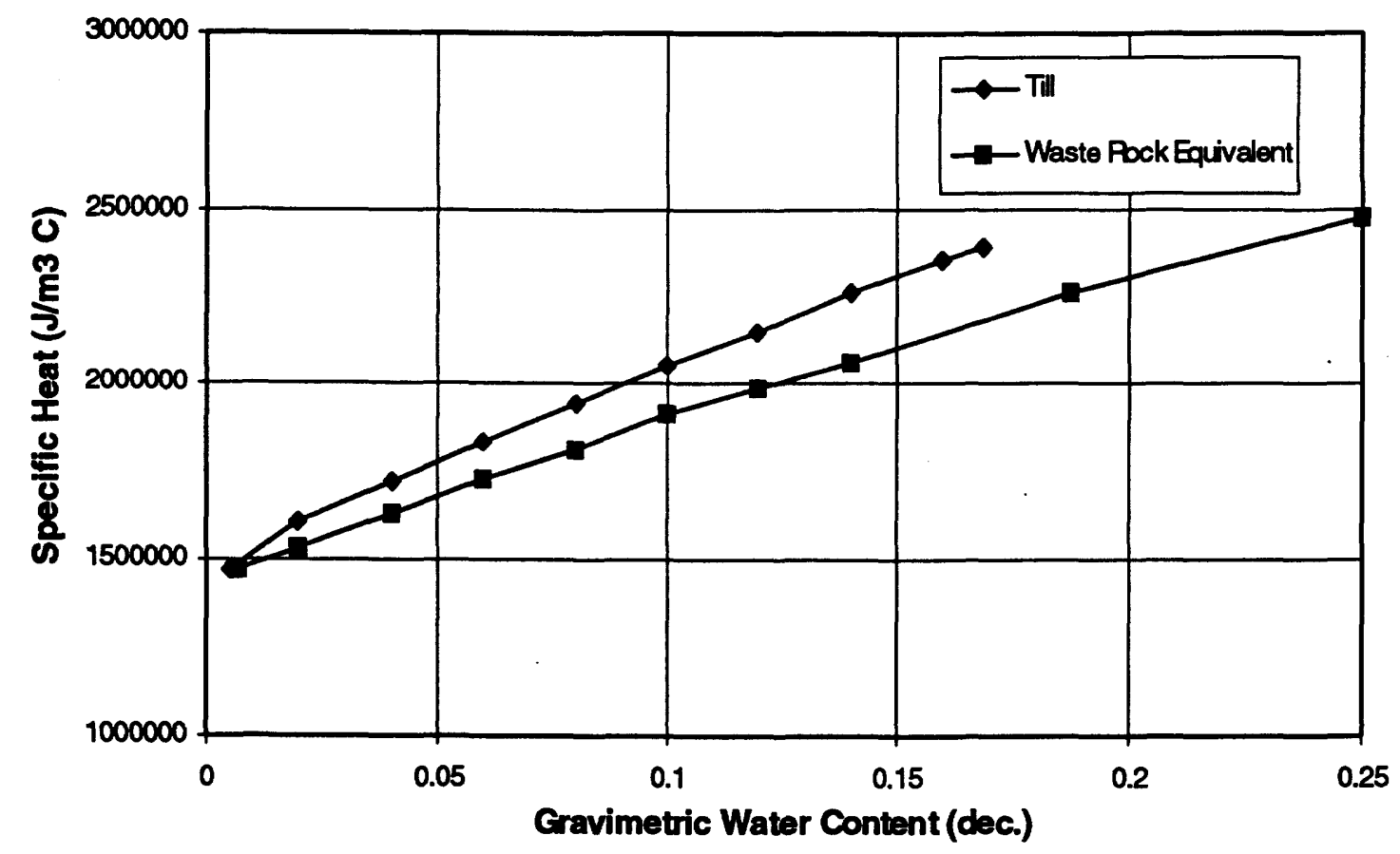

Figure A6 Specific Heat Functions of Cover Material for Non-Freezing Case (after Swanson, 1995)

\section{A3 Modelling Equity Mine Field Data}

The geometry of the soil cover system used for modelling consisted of three layers: $30 \mathrm{~cm}$ of loose till over $50 \mathrm{~cm}$ of compacted till, over $100 \mathrm{~cm}$ of waste rock (i.e., simulated using Beaver Creek Sand). The finite element mesh had 33 nodes, with nodal spacings ranging from $2 \mathrm{~cm}$ at the soil surface, to $10 \mathrm{~cm}$ at the base of the waste rock. Time steps varied from 30 seconds to 21600 seconds (i.e., 6 hours), and the system was considered to have converged if the suctions and temperatures did not change by more than $1 \%$ between successive iterations.

Swanson (1995) presents field response modelling results over a time period between the start of May and the end of October, 1993, when freezing temperatures were not encountered. Field weather data collected by O'kane (1995) shows that air and ground 
temperatures begin to fall below $0^{\circ} \mathrm{C}$ in early November. The air temperature remains below $0^{\circ} \mathrm{C}$ until some time in late April or early May. Air temperature data collected by O'kane (1995) for the winter of 1993 / 1994 are presented in Figure A7. The modelling of the field data was carried out over the time period shown in Figure A7.

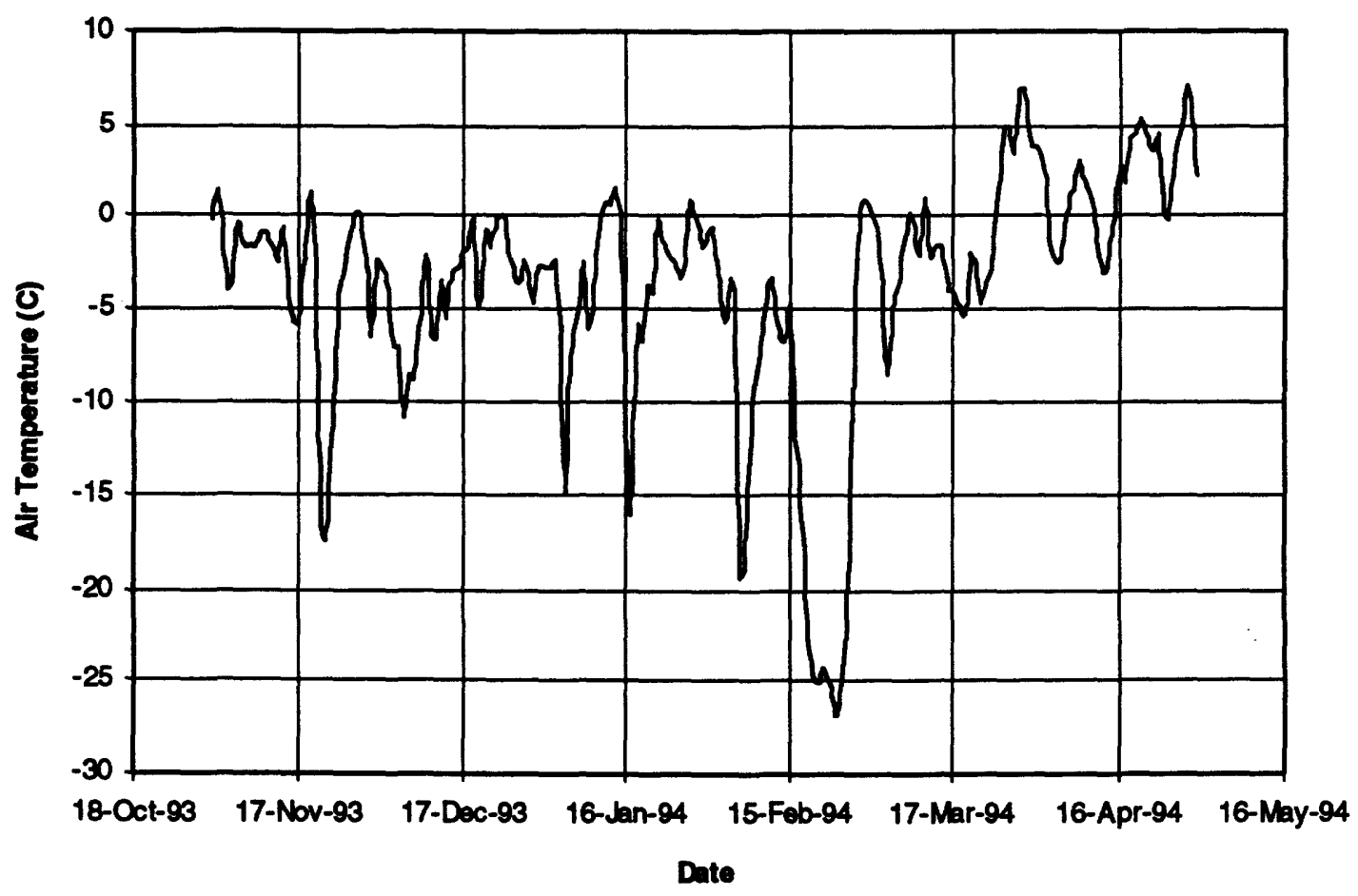

Figure A7 Mean Daily Air Temperatures Over Winter of 1993 / 1994 At Weather Station On Top of Main Waste Rock Dump

The non-freezing version of SoilCover (MEND, 1993) uses air temperature, wind speed, relative humidity, and net radiation to compute the soil temperature at the surface. These computations can only be made in the current version of SoilCover if the soil surface is not covered by snow. A hydrology report prepared for Equity Silver Mines indicates the equivalent of $370 \mathrm{~mm}$ of water falls as snow during the winter months at the mine site, and approximately $40 \%$ of this value is lost due to melting or sublimation over the winter (Ker, Priestman, 1983). The remaining $60 \%$ melts during the spring thaw. The modified freeze / thaw version of SoilCover does not include heat and mass transfer across a snow 
layer, therefore it was necessary to make some assumptions about the soil surface boundary conditions. These are subsequently discussed.

Figure A8 shows measured soil temperatures at depths of $5 \mathrm{~cm}, 10 \mathrm{~cm}$, and $31 \mathrm{~cm}$ for the winter of $1993 / 1994$. Comparison of the soil temperature profile at $5 \mathrm{~cm}$ with the air temperatures from Figure $\mathrm{A} 7$ reveals that the soil temperature dropped below $0^{\circ} \mathrm{C}$ during a cold period in the fall, and again during another period in the late spring. These events most likely occurred because the snow pack in the early winter and late spring was not thick enough to act as an insulating material preventing heat loss from the soil. Figure A8 reveals that the soil remained above freezing during the majority of the winter, and also that there was little temperature difference between $5 \mathrm{~cm}$ and $10 \mathrm{~cm}$ depths.

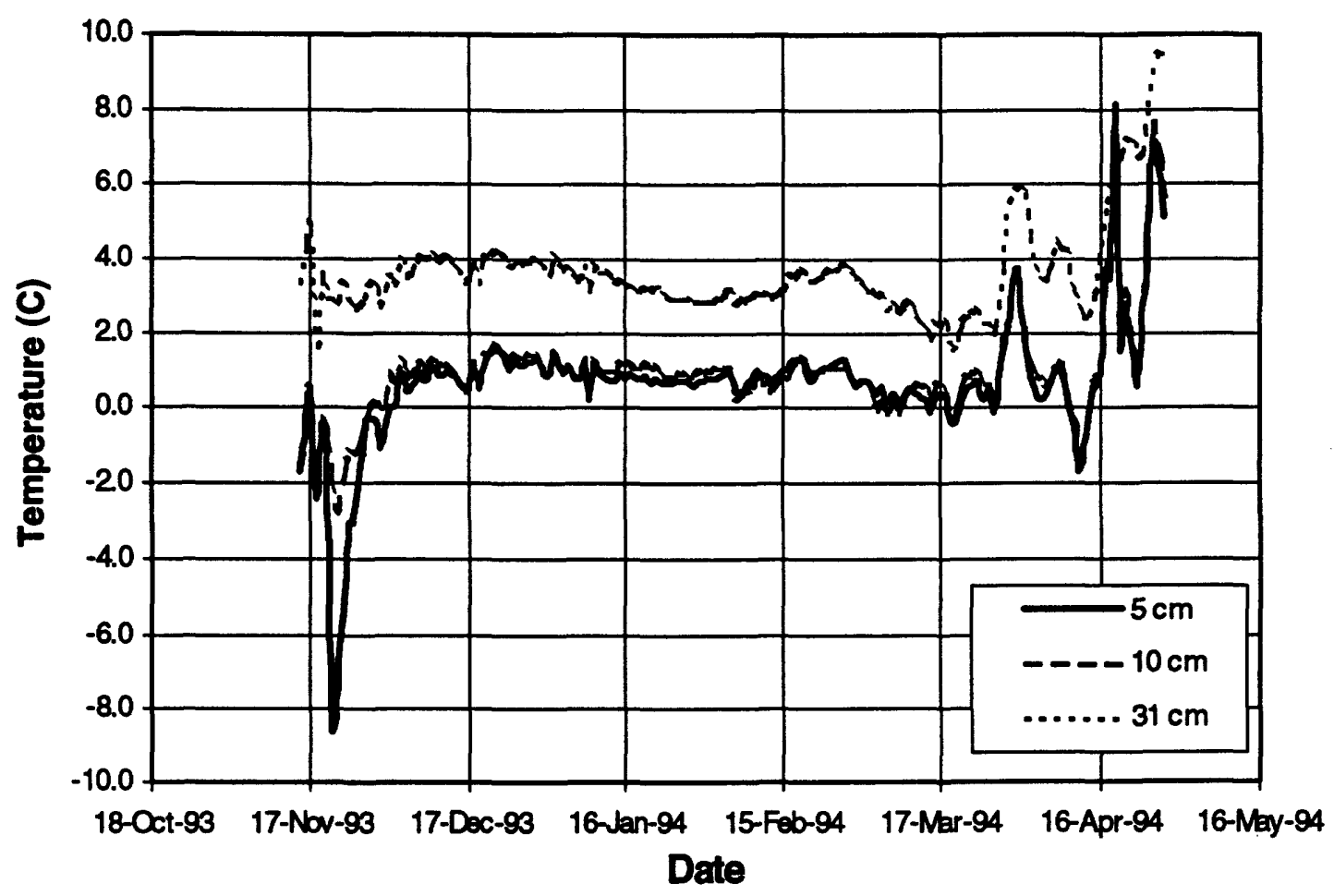

Figure A8 Measured Over Winter Soil Temperatures at Three Different Depths 
In order to verify that the modified SoilCover (MEND, 1993) program can model freeze and thaw behaviour in the soil it was necessary to impose temperature and flux boundary conditions on the system. No attempt was made to have the computer model predict energy and evaporative fluxes at the surface. The surface temperature was set to be $0.5^{\circ} \mathrm{C}$ colder than the $5 \mathrm{~cm}$ measured temperature. This seemed reasonable given that there was almost no difference in temperature between $5 \mathrm{~cm}$ and $10 \mathrm{~cm}$ depths. The warm end temperature at the surface of the waste rock (i.e., at a depth of $1.5 \mathrm{~m}$ ) was assumed constant at $25^{\circ} \mathrm{C}$ as suggested by Swanson (1995). Based on the hydrology report, the snow equivalent of $222 \mathrm{~mm}$ of water was assumed to infiltrate into the cover at an even rate during the last two weeks of the thaw period (i.e., April 23 to May 7).

Simulated and measured temperature profiles at two different depths over the winter of 1993 and 1994 are illustrated in Figure A9. Comparison of computed results with measured results reveals three points. First, there is excellent trend agreement between measured and computed results at the $5 \mathrm{~cm}$ depth. This should be expected given the fact that the model boundary conditions were based on the measured values near the surface. The second point to note is that there is excellent agreement between computed and measured results for all times when the soil temperature is above freezing. The third point is that there is poor agreement between measured and computed results at the $30 \mathrm{~cm}$ depth when the temperatures are below freezing and ice is present in the soil.

Figure A10 shows the ice content at a depth of $5 \mathrm{~cm}$ during the test period, and Figure A11 shows the corresponding thermal conductivity values of the soil at the same depth over the same period. 


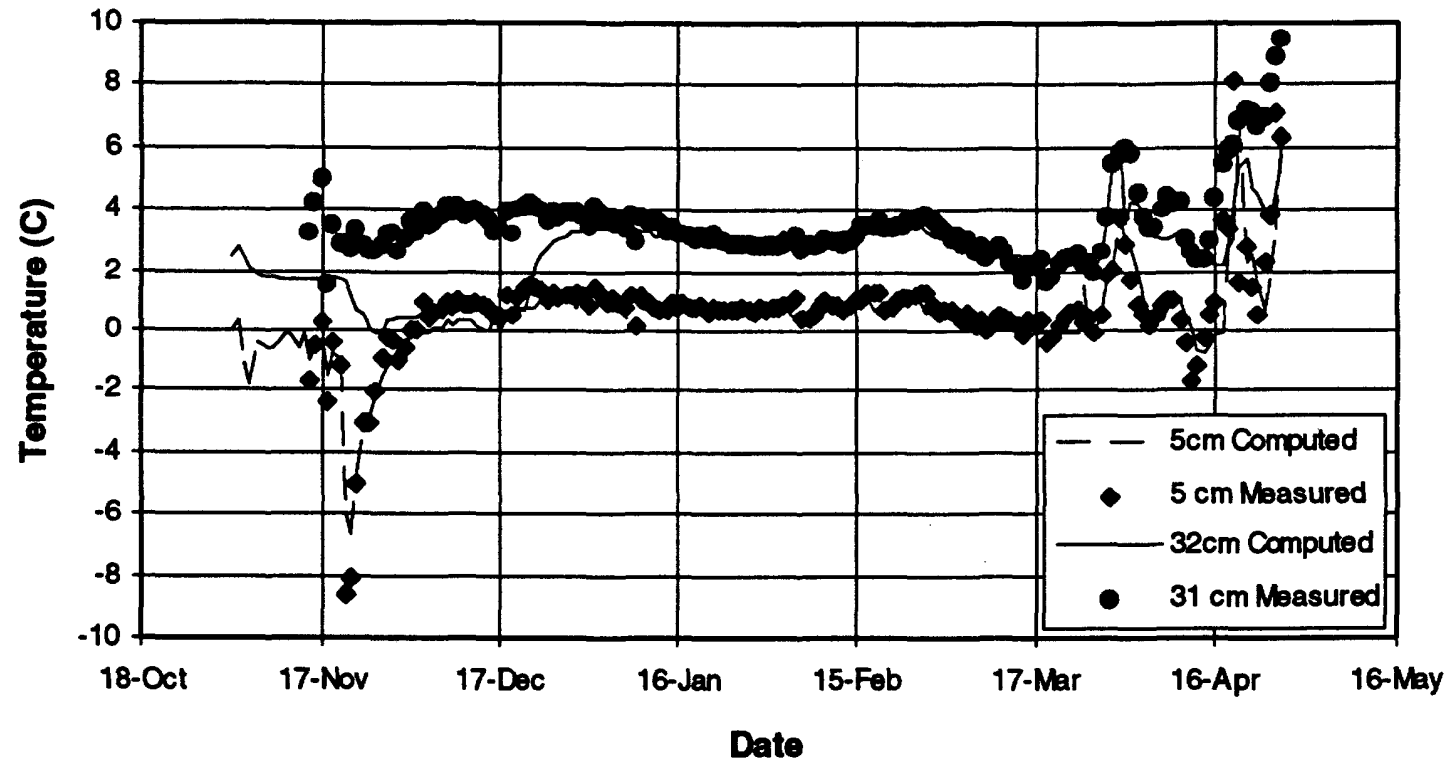

Figure A9 Computed and Measured Soil Temperatures at Two Depths During Field Data Simulation

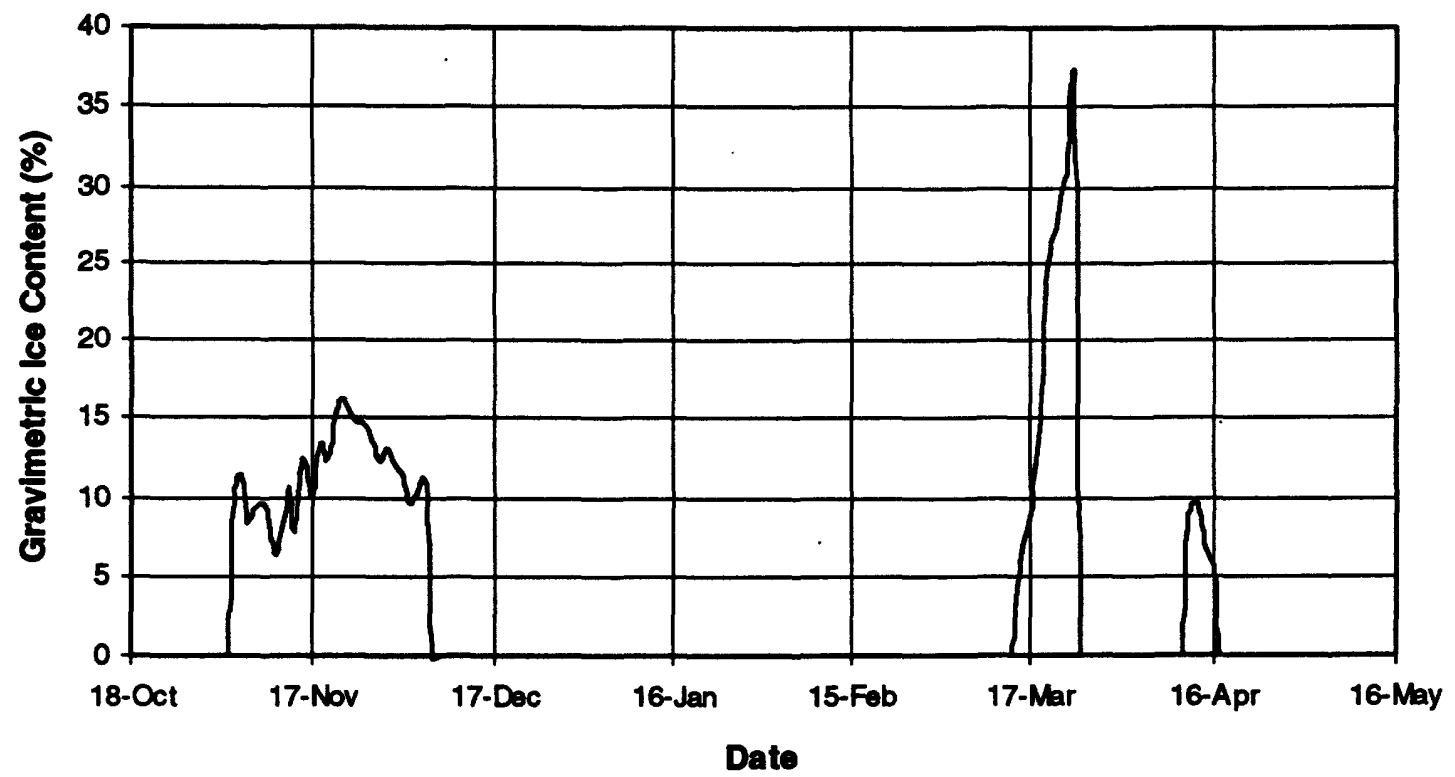

Figure A10 Computed Gravimetric Ice Contents at $5 \mathrm{~cm}$ Depth During Field Data Simulation 


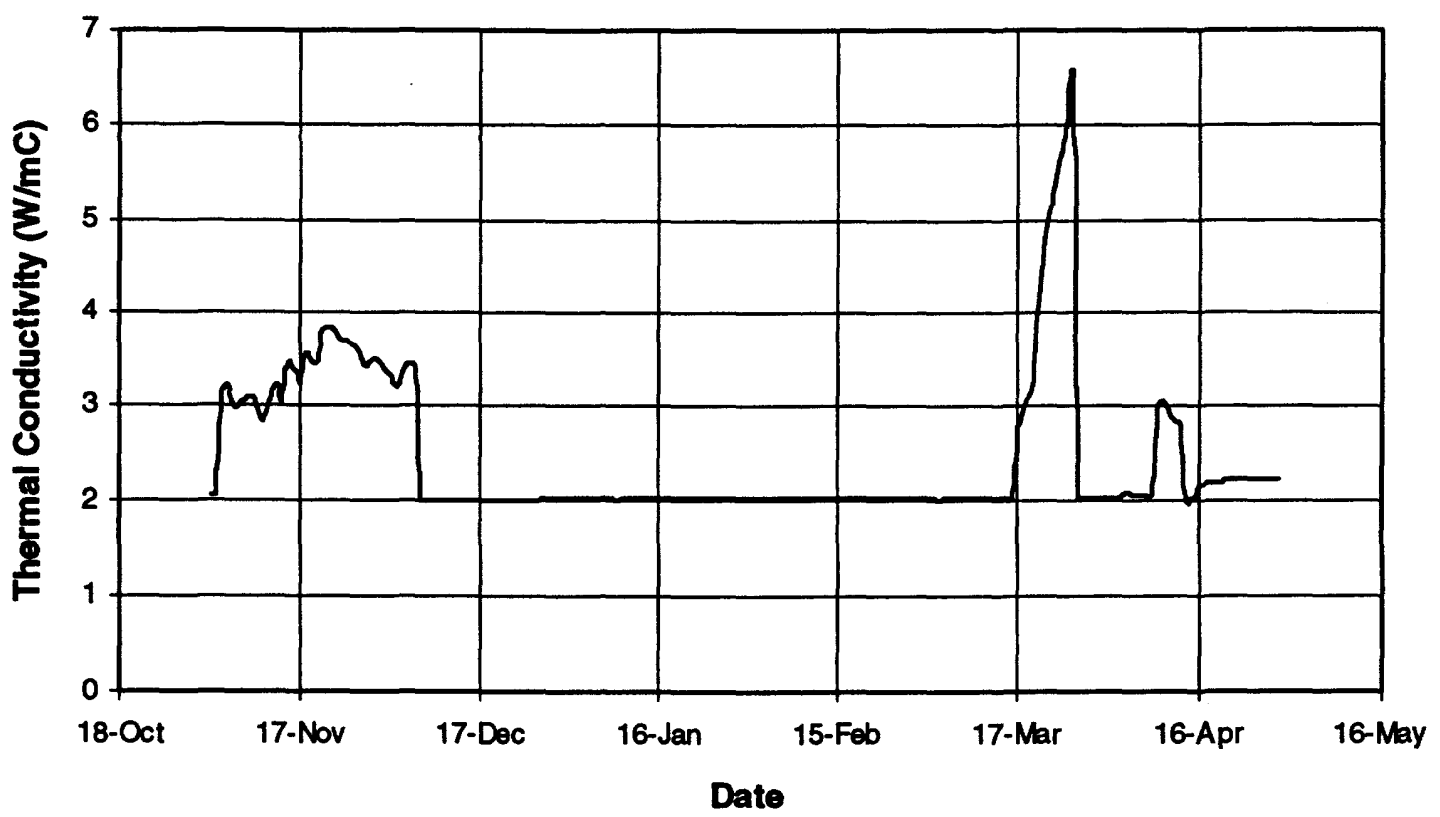

Figure A11 Computed Soil Thermal Conductivity Values at a Depth of $5 \mathrm{~cm}$ During Field Data Simulation

Figure A11 illustrates the increase in thermal conductivity values that result when ice forms in the soil. This increase is likely the reason for the poor agreement between computed and measured temperature profiles in Figure A9. When too much heat is removed from the soil system, the result is a cold front that advances faster and deeper than it should.. As a frost front advances, ice forms in some of the pores. Because ice has a higher thermal conductivity than water more heat is removed from the warmer soil. In turn, the cold front moves deeper and more water freezes.

Initial observation of the computed thermal conductivity values suggests that they may be computed incorrectly. However, this is not likely the case. There may be some error in thermal conductivity estimations based on the calculation method, but as the thermal conductivity is significantly affected by the ice content (as illustrated in Figure A11) the likely cause of disagreement between computed and measured soil temperature values is the incorrect calculation of ice content. Ice contents are computed incorrectly if the soil 
freezing curve relationship is not accurate. For example, if the soil freezing curve indicates that ice will form at $-0.05^{\circ} \mathrm{C}$ instead of $-0.5^{\circ} \mathrm{C}$ then higher ice content values will be computed as the temperature drops. In this simulation the soil freezing curve relationship was estimated using a measured soil water characteristic curve and the form of the Clapeyron equation given by Black and Tice (1989). As discussed in Chapter 2, there are problems associated with this approach. An experimentally determined soil freezing curve should yield more accurate results.

Figure A12 shows the computed liquid water contents over the duration of the test. This figure depicts the expected trends. Measured water content data near the surface during the winter months are not available because the thermal conductivity sensors used to measure matric suction do not operate effectively if pore-ice is present. It can be noted that the reductions in liquid water content values occur at the same time there is an increase in ice content (i.e., Figure A10). Figure A12 also shows that there was little redistribution of pore-water from warmer regions to colder regions as illustrated by the constant water content at a $32 \mathrm{~cm}$ depth. This is directly attributable to the lower permeability of the clay till, compared with the high permeability and high moisture flux observed in the laboratory testing of silica flour.

During the last two weeks of the simulation period, a snow water equivalent of $15 \mathrm{~mm}$ per day was applied as a flux at the top boundary. This value agrees with the snow melt predicted by Ker, Priestman (1983) in their hydrology study of the Equity Silver Mine region. A majority of the $15 \mathrm{~mm}$ of water applied each day was computed by SoilCover to be runoff. The amount which did infiltrate contributed to an increase in computed water contents to near saturation levels at the surface. This increase is reflected in Figure A12 


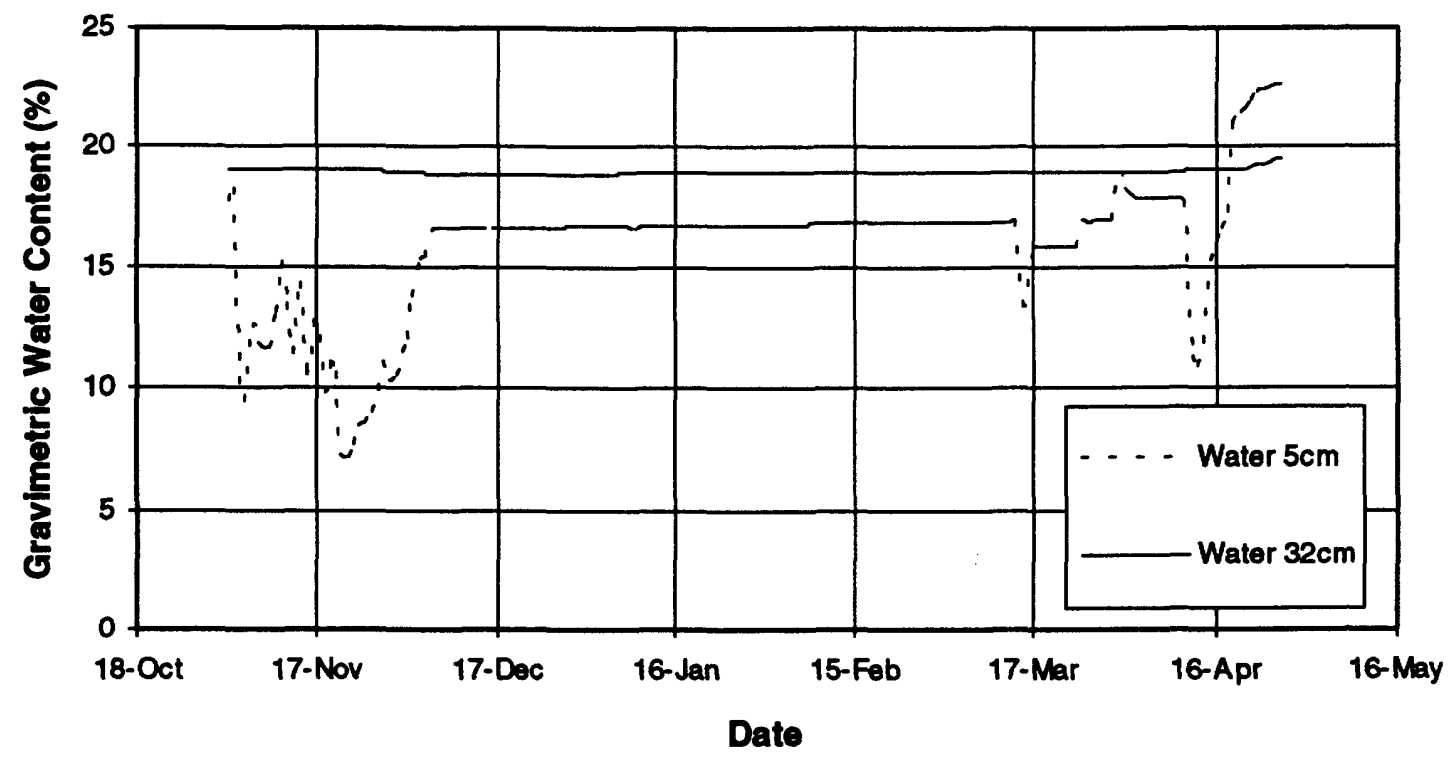

Figure A12 Computed Liquid Water Contents at Two Depths During Field Data Simulations

Finally, some comments can be made about the general trends observed in these results. The objective of a soil cover is to minimize infiltration of oxygen into the waste rock. This is done by keeping the water content in the compacted layer of the soil cover near saturation. Results of the freezing tests on the silica flour (presented earlier) clearly show that there is a large re-distribution of water within the soil. This pattern was not evident during the freezing simulations of the clay till cover and it was not expected. The permeability of the compacted clay till is sufficiently low that it limits the quantity of liquid flux over time. The soil freezing curve for the clay till shows that large quantities of liquid water are present in the soil even at temperatures as low as -5 or $-6^{\circ} \mathrm{C}$. If the majority of water did not freeze in the cover, then the matric suctions did not increase sufficiently high enough to draw water out of the warmer soils. As a result, the compacted layer of the soil cover tended to remain saturated which is the desired effect. 


\section{APPENDIX B SUBROUTINE CALL OUT DIAGRAM}


Subroutine Call Out Chart

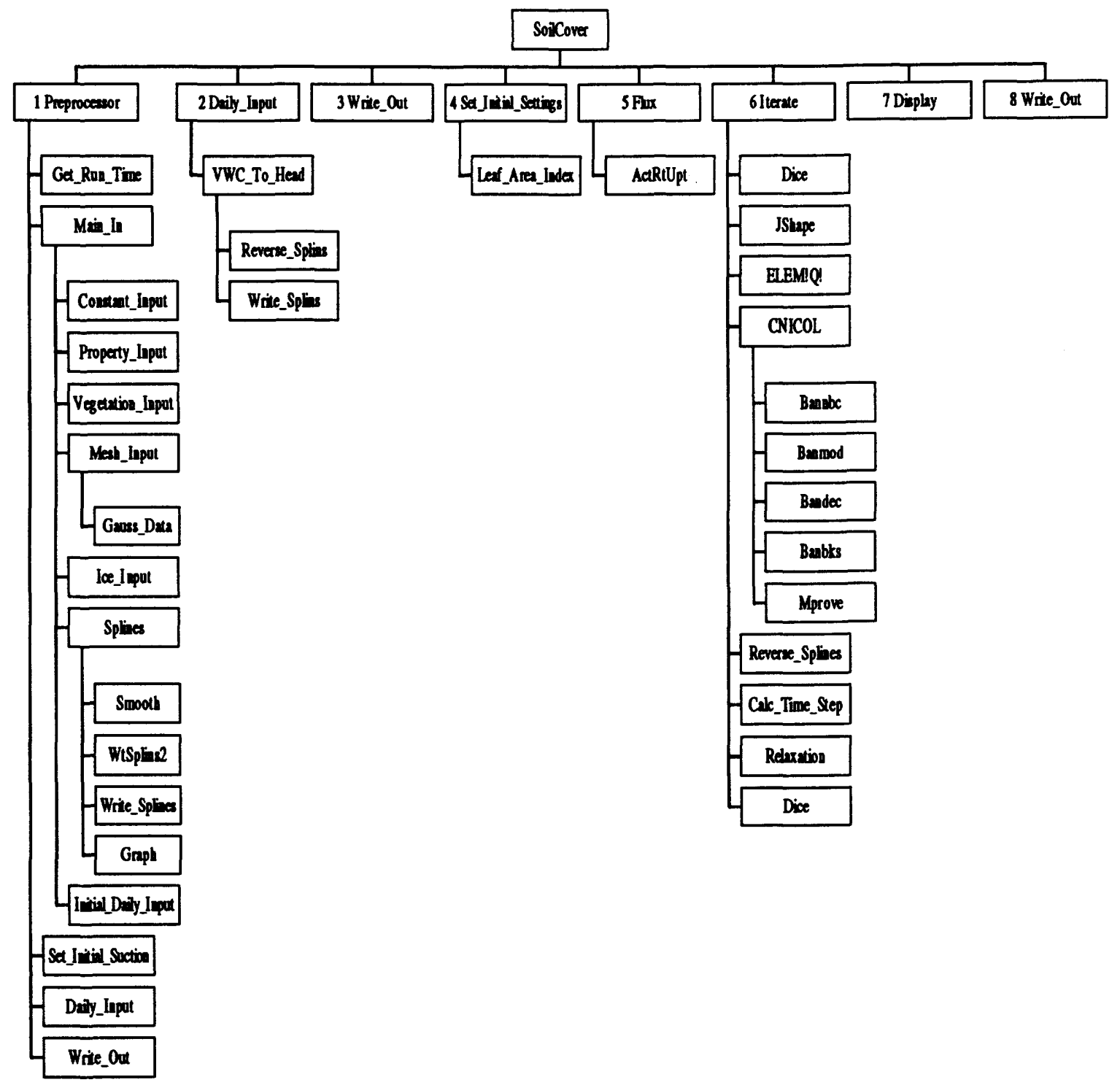




\section{APPENDIX C MAIN COMPUTER PROGRAM CODE}

Note: All revisions for freeze / thaw analysis are in bold type. 


\section{Program SoilCover}

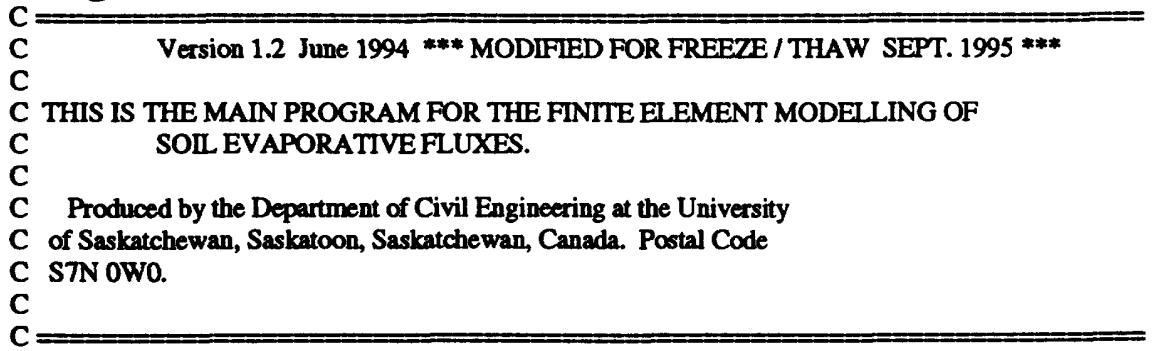

IMPLICIT NONE

INCLUDE FUNCTION.FT

INCLUDE 'DECLARE.FT INCLUDE 'SPPCOMMO.FT

! All include files in this file I Used by SPP ploting routines

C

integer dayclose ! flag used to close at end of day prematurely

REAL Lapsed_Time ! Total run time in seconds

character* 2 hotkey ! hot key to close SoilCover prematurely

INTEGER ivid,i

LOGICAL printed,out

REAL water

C REAL WaterBalance ! $(\mathrm{mm} /$ day) Function to Calculate the WaterBalance

C

C Write out program information header

C

WRITE(*,*)

WRITE(*,*)

WRTTE $(*, *) \cdot$

WRITE $(*, *)^{\prime}$

WRTTE(*,*)'

WRITE(*,*)'

WRITE(*,*)'

WRTTE $(*, *) \cdot$

WRTTE(*,*)'

WRITE(*,*)

C

C

C Start the preprocessor to do all the preliminary work which includes

C getting the runtime settings, reading in the data files,

C setting the initial suctions and temperatures, and writing the

C initial conditions to the output file.

C

TTIME $=0.0 \mathrm{DO}$

DAYCLOSE $=1$

AEsum $=0.0 \mathrm{DO}$

PEsum $=0.0 \mathrm{DO}$

ATsum $=0.0 \mathrm{DO}$

PTsum $=0.0 \mathrm{DO}$

RunOff $=0.0 \mathrm{DO}$

DO $\mathrm{i}=1$,NNODES

SFLUX (i) $=0.0 \mathrm{DO}$

SFLUXI (i) $=0.0 \mathrm{DO}$

SFLUXV (i) $=0.0 D 0$

SFLUXARU(i) $=0.0 \mathrm{DO}$

SFLUXPRU(i) $=0.0 \mathrm{DO}$

ENDDO

MAXD_OUT_TODAY $=0.0$ ! Clear daily maxd_out counter

CALL PREPROCESSOR

out $=$ true

C

DO NDAY=1,DAYS 


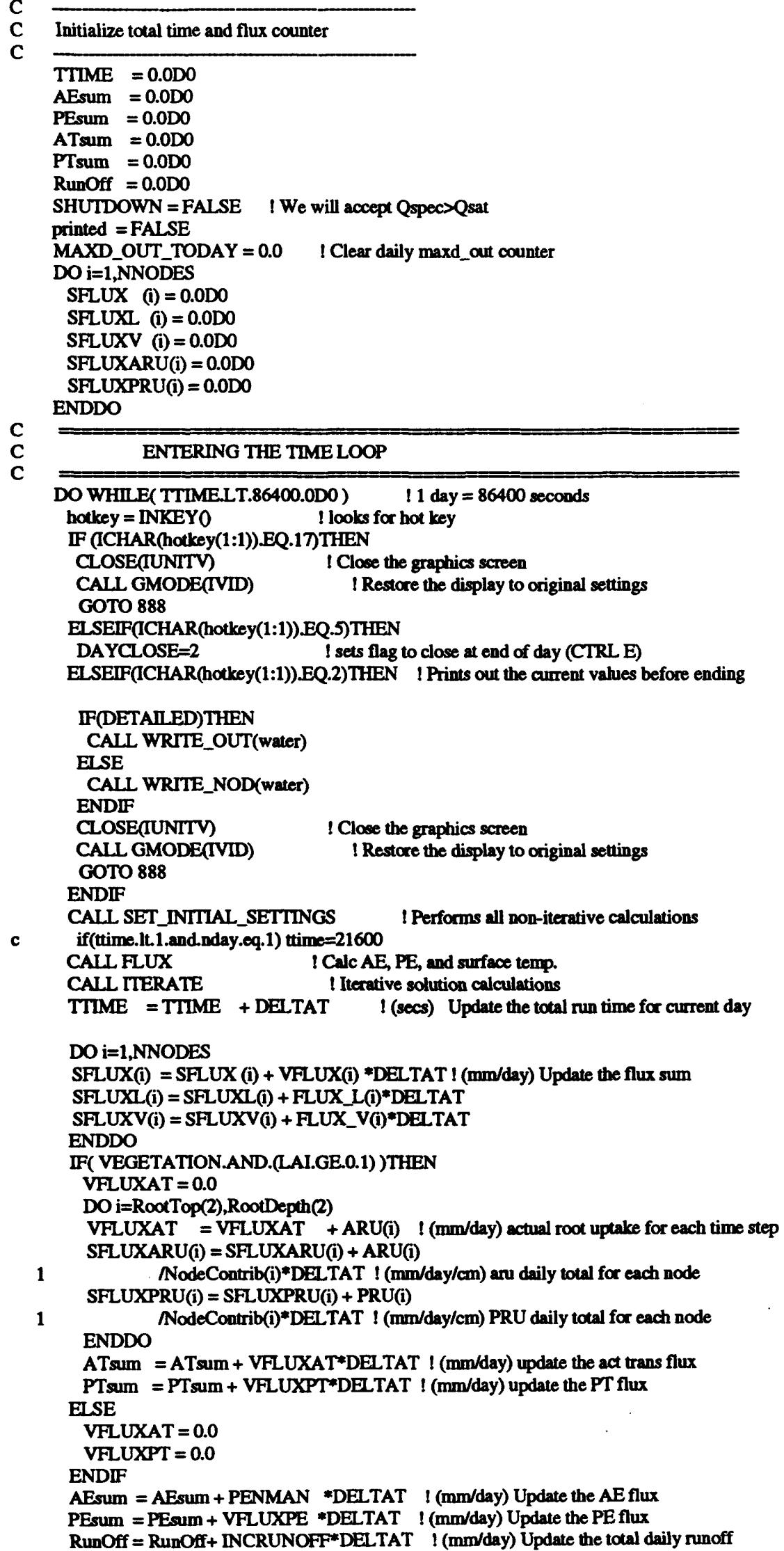


water $=$ WaterBalance $0 \quad !(\mathrm{mm} /$ day $)$ Calculate the current water balance

IF(GRAPHICS) THEN

CALL DISPLAY(ivid, VFLUXPE,PENMAN,VFLUXAT,RAIN, water)

ENDIF

IF(.NOT.printed.AND.PrintTime.EQ.1)THEN ! Print output at noon

If(TTIME.GE.43200.D0)THEN

printed $=$ TRUE

IF(DETAIIED)THEN

CALL WRITE_OUT(water)

ELSE

CALL WRITE_NOD(water)

ENDIF

ENDIF

ENDIF

C write( $\left({ }^{*}, *\right)$ 'day,ksat, ttime',nday,satk(3),ttime

if(nday.eq.1 and. ttime.ge. 21600 and.

1 out and. ttime.le.22600) then 16 hour output

call write_nod(water)

out=?lse

elseif(nday.eq. 1 and. ttimege.43200 and. not.out)then ! 12 hour output

call write_nod(water)

out=true

endif

ENDDO ! DO WHILE( TTIME.LT.86400.0DO)

C FINISHED THE TIME LOOP

C

IF(PrintTime.EQ.2)THEN

! Print cutput at midnight

IF(DETAIIED)THEN

CALL WRITE_OUT(water)

ELSE

CALL WRITE_NOD(water)

ENDIF

ENDIF

C Resetting the time step to the initial time step specified

C to reduce the shock on the system induced by the new bondary

C conditions applied on a new day.

C DEI TAT = FIRST DEITAT

IF(GRAPHICS)THEN

CLOSE(IUNITV)

CALL GMODE(IVID)

ENDIF

IF(DAYCLOSE.EQ.2)THEN ICloses SoilCover due to hot key

CLOSE(IUNITV) ! Close the graphics screen

CALL GMODE(IVID) I Restore the display to original settings

GOTO 888

ENDIF

ENDDO ! DO NDAY = 1,DAYS

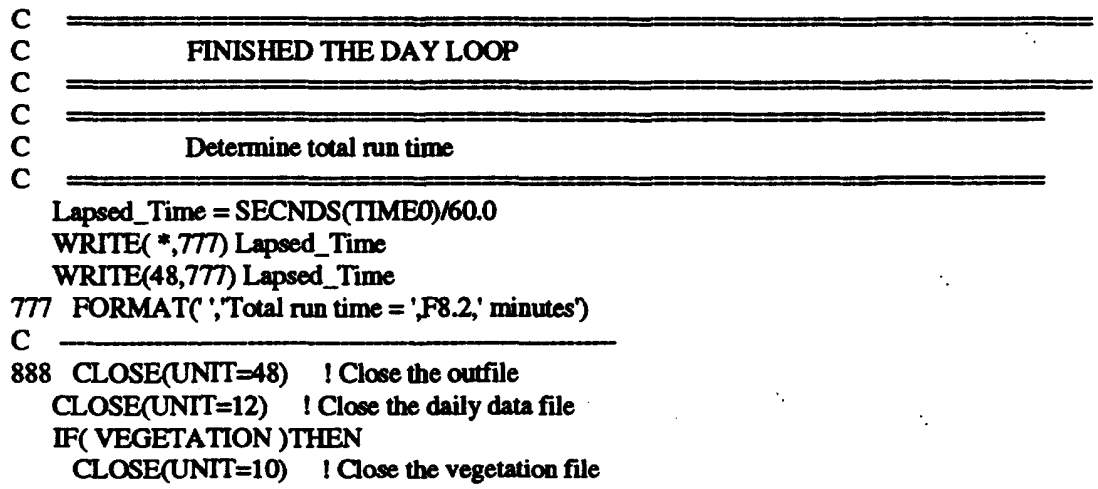


ENDIF

C END ! Of program SoilCover 


\section{APPENDIX D SUBROUTINE CODE}


C This subroutine calculates the actual root uptake for each node

C SUBROUTINE ActRtUpt(node) !(mm/sec)

C IMPLICIT NONE INCLUDE FUNCTION.FT INCLUDE DECLAREFT

! Ensure that all variables have been correctly defined ! Contains all FUNCTION declarations

! Contains all common block declarations

C INTEGER node ! (HRS) Number of hours in a day REAL limitFactor! Plant Limiting Factor

limitFactor $=$ Calc_PlantLimitFactor $($ SUCNOD(node)) $!$ calcs PLF ARU(node) $=$ PRU(node) * limitFactor

C $=$ END

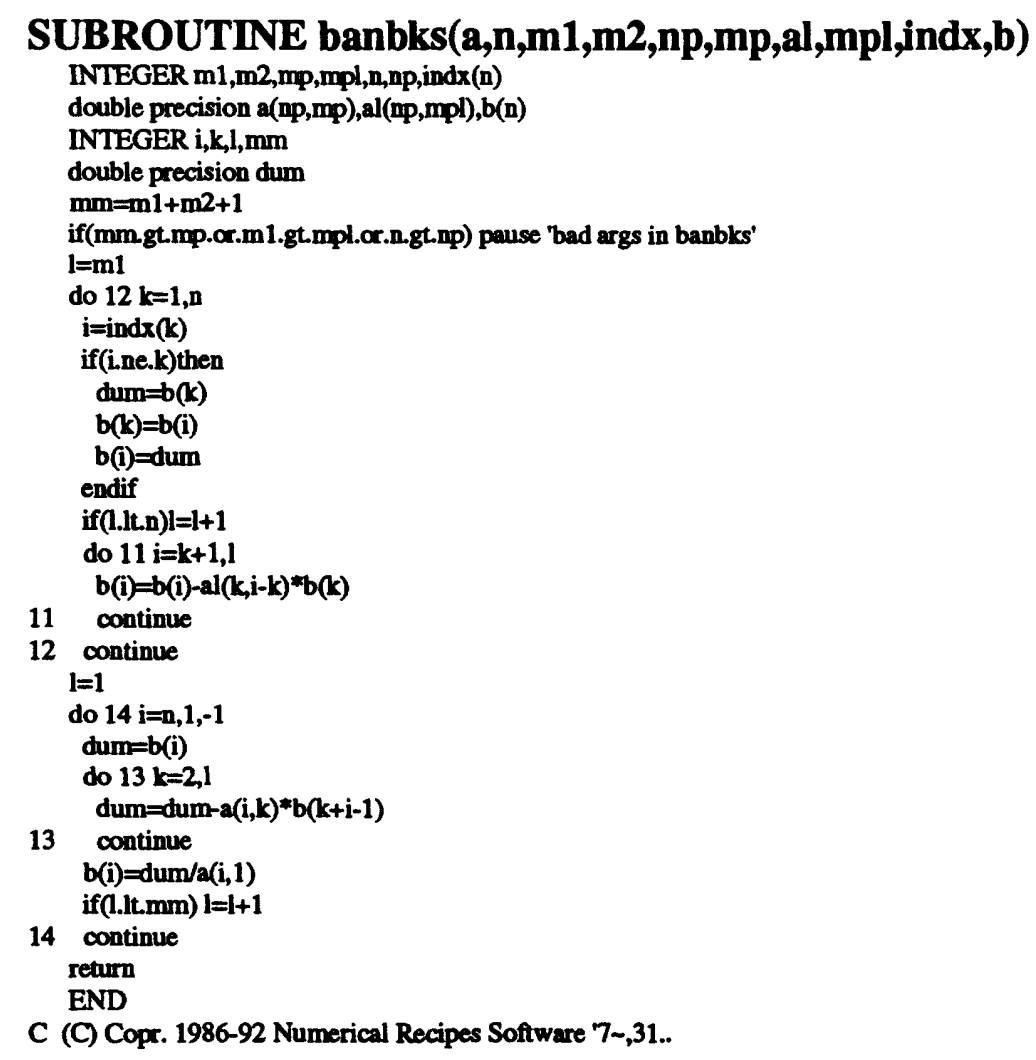

\section{SUBROUTINE bandec(a,n,m1,m2,np,mp,al,mpl,indx)}

INTEGER m1,m2,mp,mpl,n,np,indx(n)

double precision a(np,mp),al(np,mpl), TINY

PARAMETER (TINY=1.D-20)

INTEGER i,j,k,l,mm

double precision dum

$m m=m 1+m 2+1$

if(mm.gt.mp.or.m1.gt.mpl.or.n.gt.np) pause 'bad args in bandec'

$\mathbf{l}=\mathbf{m} 1$

do $13 \mathrm{i}=1, \mathrm{~m} 1$

do $11 \mathrm{j}=\mathrm{m} 1+2-\mathrm{i}, \mathrm{mm}$ 


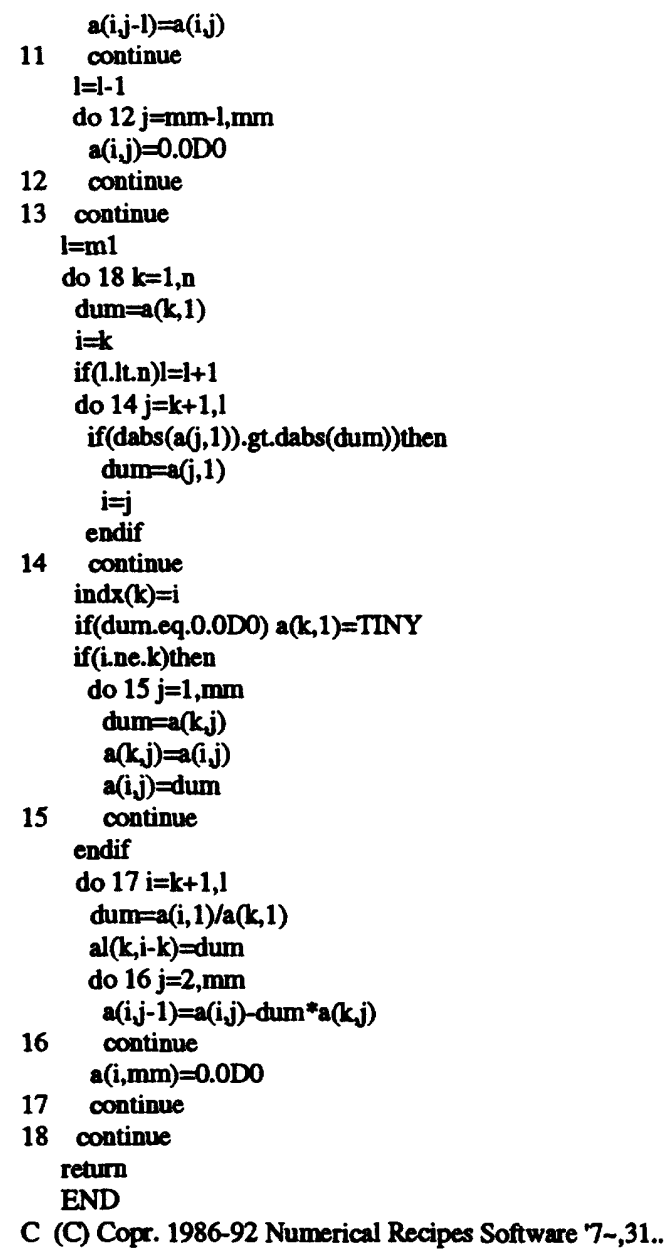

\section{SUBROUTINE BANMOD(a,n,m1,np,mp,b)}

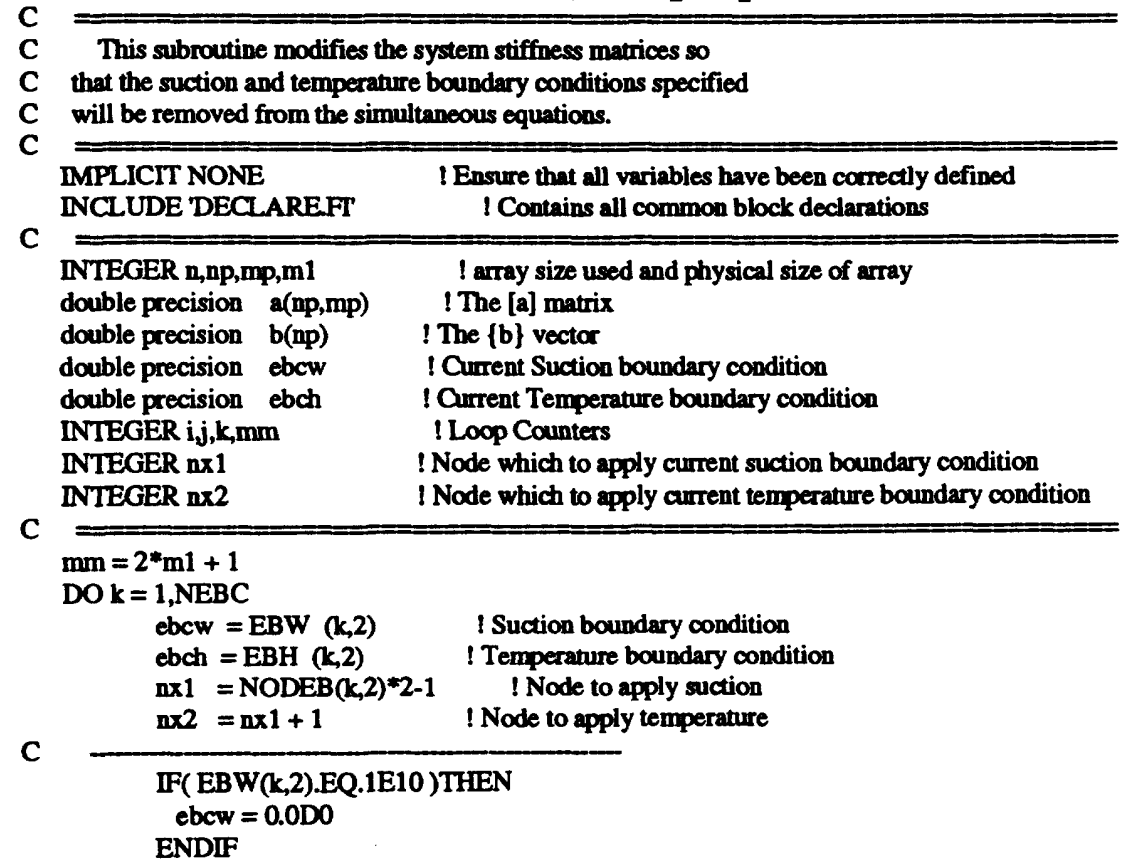



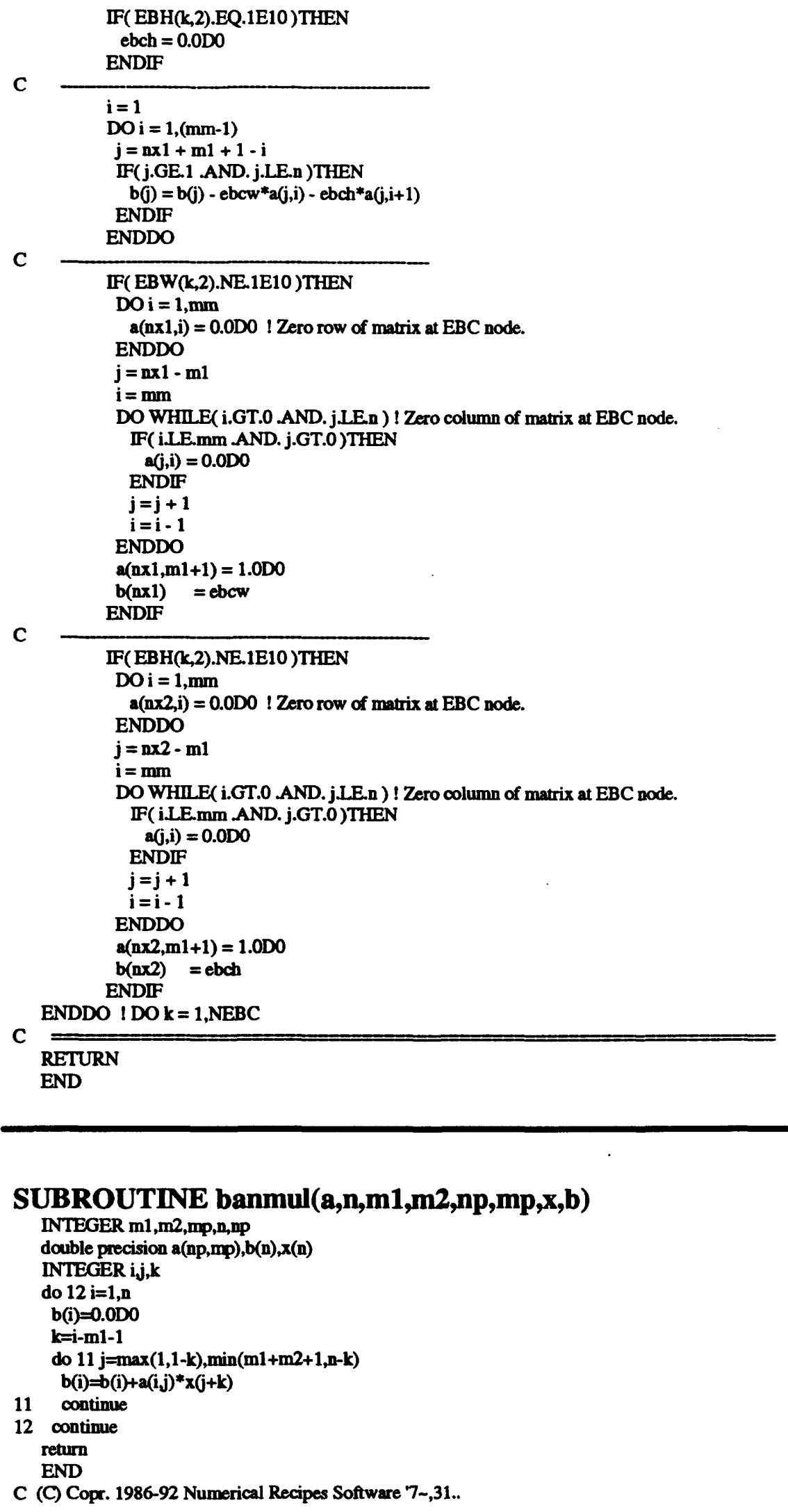


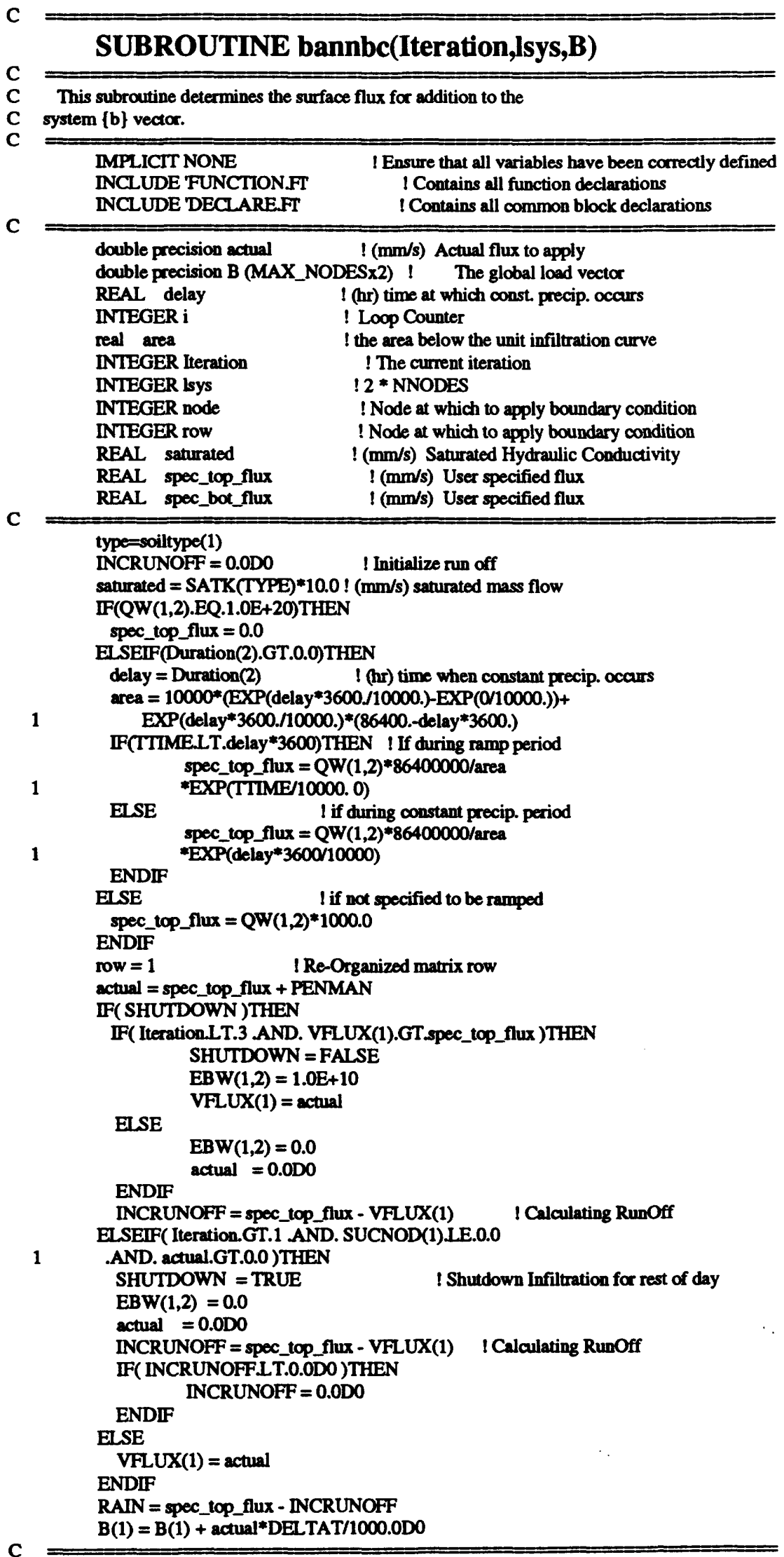


IF(QW(2,2).EQ.1.0E+20)THEN

spec_bot_flux $=0.0$

EISE

spec_bot_flux $=Q W(2,2) * 1000.0$

VFLUX(NNODES) $=$ spec_bot_flux

row $=2 *$ NNODES -1 ! Re-Organized matrix row

$\mathrm{B}($ row $)=\mathrm{B}($ row $)+$ spec_bot_flux $*$ DELTAT $/ 1000.0 \mathrm{D0}$

ENDIF

C Apply root uptake flux over the nodes throughout the root depth

C if a vegetation was specified

C

IF( VEGETATION.AND.(LAI.GE.0.1))THEN

DO $\mathrm{i}=$ Rook Top(2), RootDepth(2) I Add in act root uptake

row $=2 * \mathrm{i}-1$

ENDDO

$B($ row $)=B($ row $)+A R U(i) * D E L T A T / 1000.0 D 0$

ENDIF

RETURN

END

C

SUBROUTINE CALCULATE_TIME_STEP

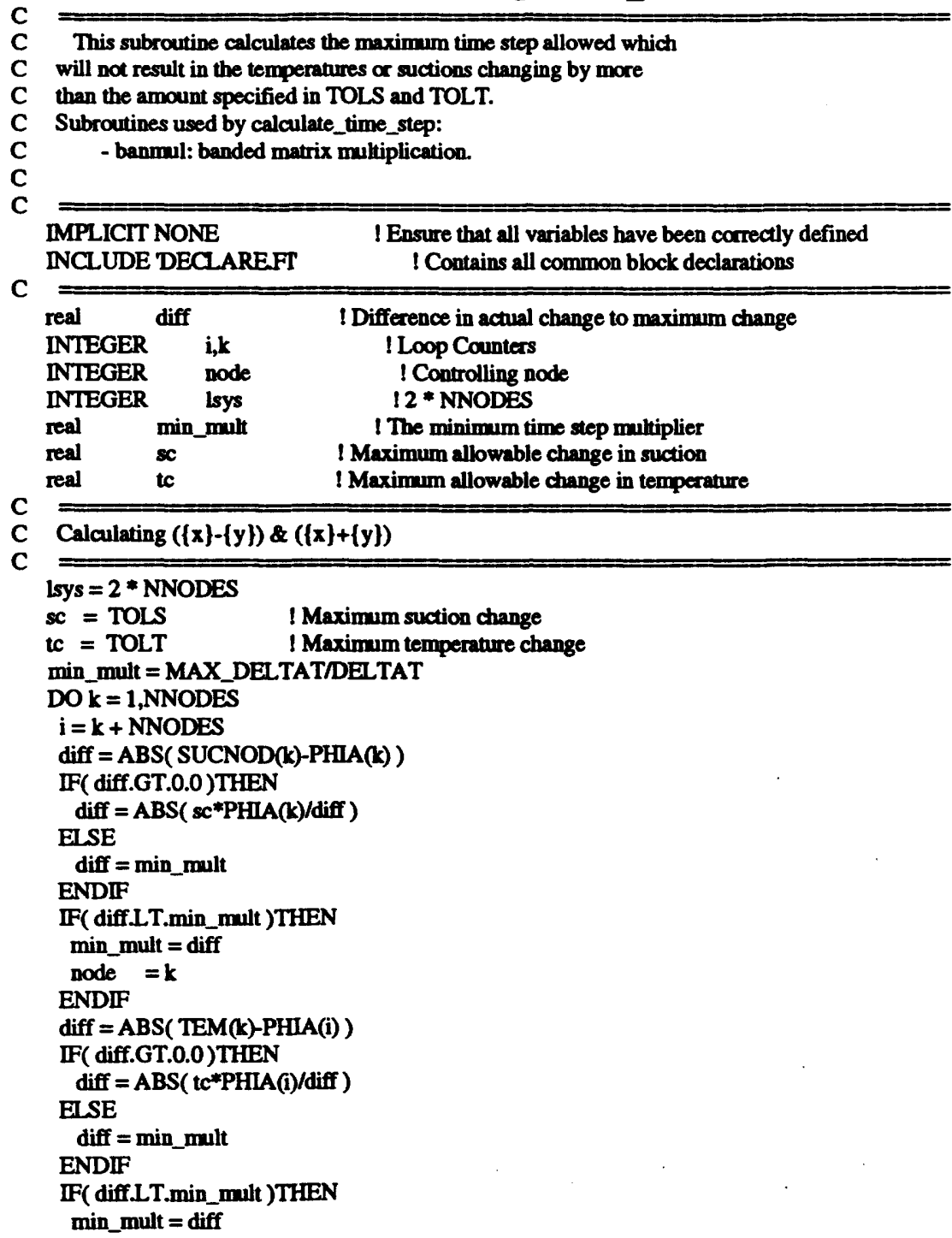




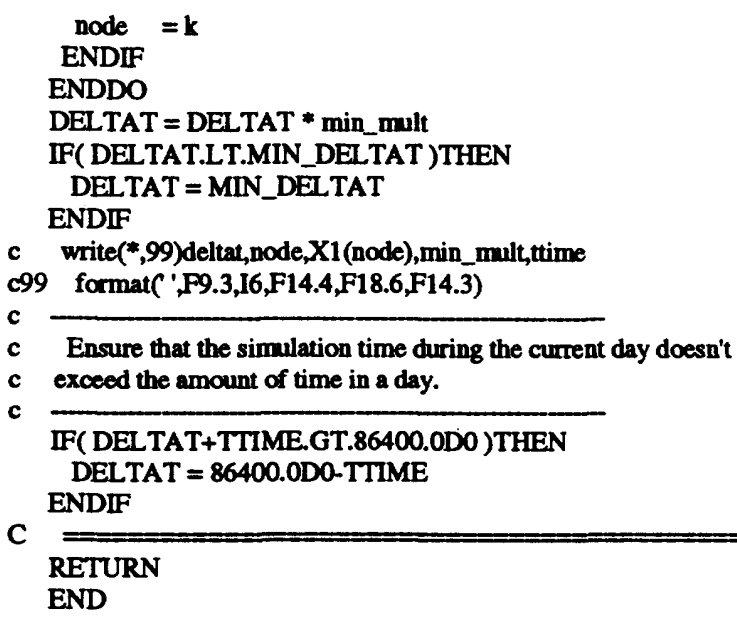

\section{C}

\section{SUBROUTINE CNICOL(Iteration)}

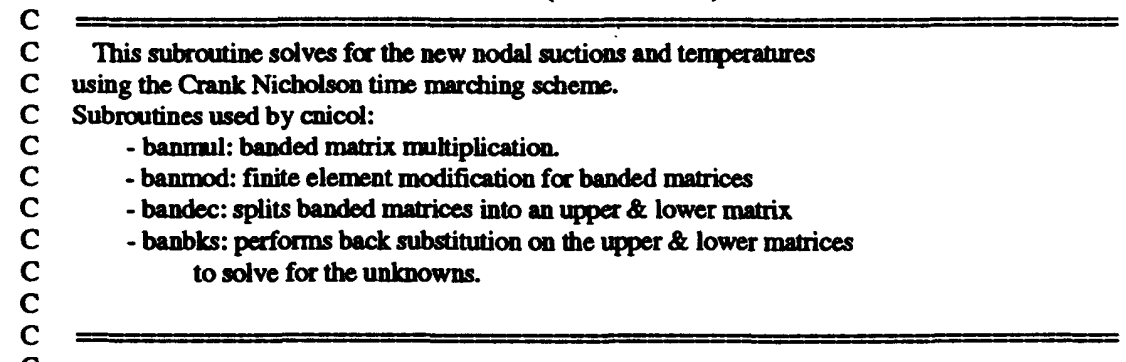

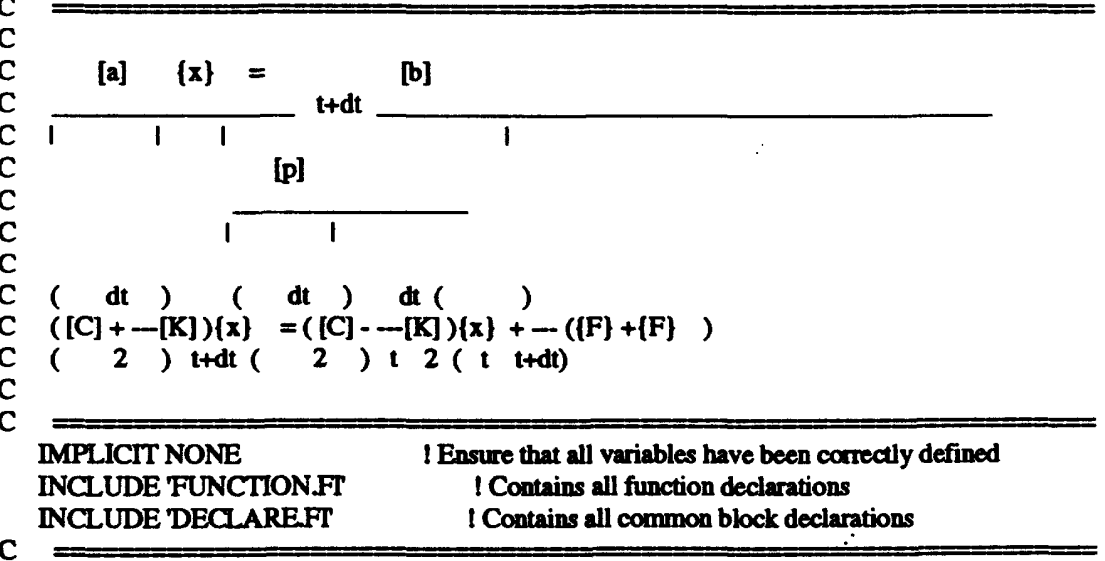

$$
\text { INTEGER MAX_BAND }
$$

c integer soil

PARAMETER(MAX_BAND = 11) IFor a quadratic element

DOUBLE PRECISION THETA,THETA1

PARAMETER( THETA $=0.5 \mathrm{DO}$ )

PARAMETER( THETA1 = 1.DO-THETA)

C

double precision a (MAX_NODESx2,MAX_BAND) ! The banded ' $a$ ' matrix where $[a]\{x\}=\{b\}$

double precision au (MAX_NODESx2,MAX_BAND) I The upper half of the lu decomposition of ' $a$ ' double precision al MAX_NODESx2,5 ) IThe lower half of the tu decomposition of ' $a$ '

double precision $b$ (MAX_NODESx2) IThe ' $b$ ' vector where $[a]\{x\}=\{b\}$

double precision fo (MAX_NODESx2) I The loed vector (@t )

double precision $\mathrm{f1}$ (MAX_NODESx2) I The load vector ( $1 \mathrm{t}+\mathrm{dt}$ )

INIEGER indx(MAX_NODESx2) ! Used by Numerical Recipies bandec \& banksb \& mprove

INTEGER Iteration

INTEGER i,j,k,l,m,type

INTEGER $\mathrm{m} 1, \mathrm{~m} 2, \mathrm{~mm}$

INTEGER lsys

! The current iteration

! Loop Counters

! Lower and Upper Half Band Widths, and total band width

!2* NNODES 
double precision stor(MAX_NODESx2,MAX_BAND) ! The banded version of the storage matrix.

double precision p (MAX_NODESx2,MAX_BAND) ! Matrix $=[C]-(d t / 2) *[K]$ NOTE: This matrix has been rearranged and banded

double precision stif(MAX_NODESx2,MAX_BAND) ! The banded version of the stiffness matrix.

double precision $x$ (MAX_NODES 2 ) I The ' $x$ ' vector where $[a]\{x\}=\{b\}$

save fo,fl ! This must be saved between calls

C Forming the banded versions of the load vector, the vector of

C knowns, the stiffness matrix, and the storage matrix.

IF( PNODES.EQ.2)THEN ! Linear Element

$\mathrm{m} 1 \mathbf{=} 3$

$m 2=3$

ELSE

$\mathrm{ml}=5$

$m 2=5$

ENDIF

$m m=m 1+m^{2}+1$

lsys $=2 *$ NNODES

IF( Iteration.EQ.0)THEN

$\mathrm{DO} \mathrm{i}=1$, lsys

fO(i) $=\mathbf{f 1}(\mathbf{i})$

ENDDO

ENDIF

DO $\mathrm{j}=1$, lsys

$1=2 * j-1$

IF(1.GT.lsys)THEN

! Re-Organized matrix column

! Temperature related

$1=1$ - lsys +1

ENDIF

$\mathbf{f 1}(\mathrm{l})=\mathrm{SYSF}(\mathrm{j})$

! Load vector $1 \mathrm{t}+\mathrm{dt}$

IF( $\mathrm{j}$.GT.NNODES ) THEN

$x(\mathbf{l})=$ PHIA $(j)$

IThen this is temperature

ELSE

$x(I)=-P H I A(j) \quad !(x)$ vector $@ t$

ENDIF

DO $\mathrm{i}=1$,lsys

$k=2 * i-1$

! Re-Organized matrix row

IF(k.GT.lsys)THEN

1 Temperature related

$k=k-1 s y s+1$

ENDIF

$m=(1+m 1+1-k)$

Banded matrix row

IF( m.GE.1 AND. mLE.mm)THEN ! If row falls within the band

stif(k,m) = SYSTIF $(i, j)$

stor $(k, m)=$ Lump $(i, j, m 1, m 2,1 s y s)$

ENDIF

ENDDO

ENDDO

IF( Iteration.EQ.0 .AND. TTIME.EQ.0.0D0 .AND. NDAY.EQ.1 )THEN

DO $i=1,1$ sys

fo(i) $=$ f1(i)

ENDDO

ENDIF

C FORMING THE a AND $p$ MATRICES(REF:SEGERLIND)

IF( TRANSIENT)THEN

do $\mathrm{m}=1, \mathrm{~mm}$

do $k=1$, lsys

$a(k, m)=s t o r(k, m)+$ THETA *DELTAT* stif(k,m)

$p(k, m)=$ stor $(k, m)-$ THETA ${ }^{*}$ DEL TA T*stif $(k, m)$

enddo

enddo

ELSE

do $\mathrm{m}=1, \mathrm{~mm}$

do $\mathrm{k}=1$, lsys

$\mathbf{a}(\mathbf{k}, \mathbf{m})=\operatorname{stif}(\mathbf{k}, \mathbf{m})$ 


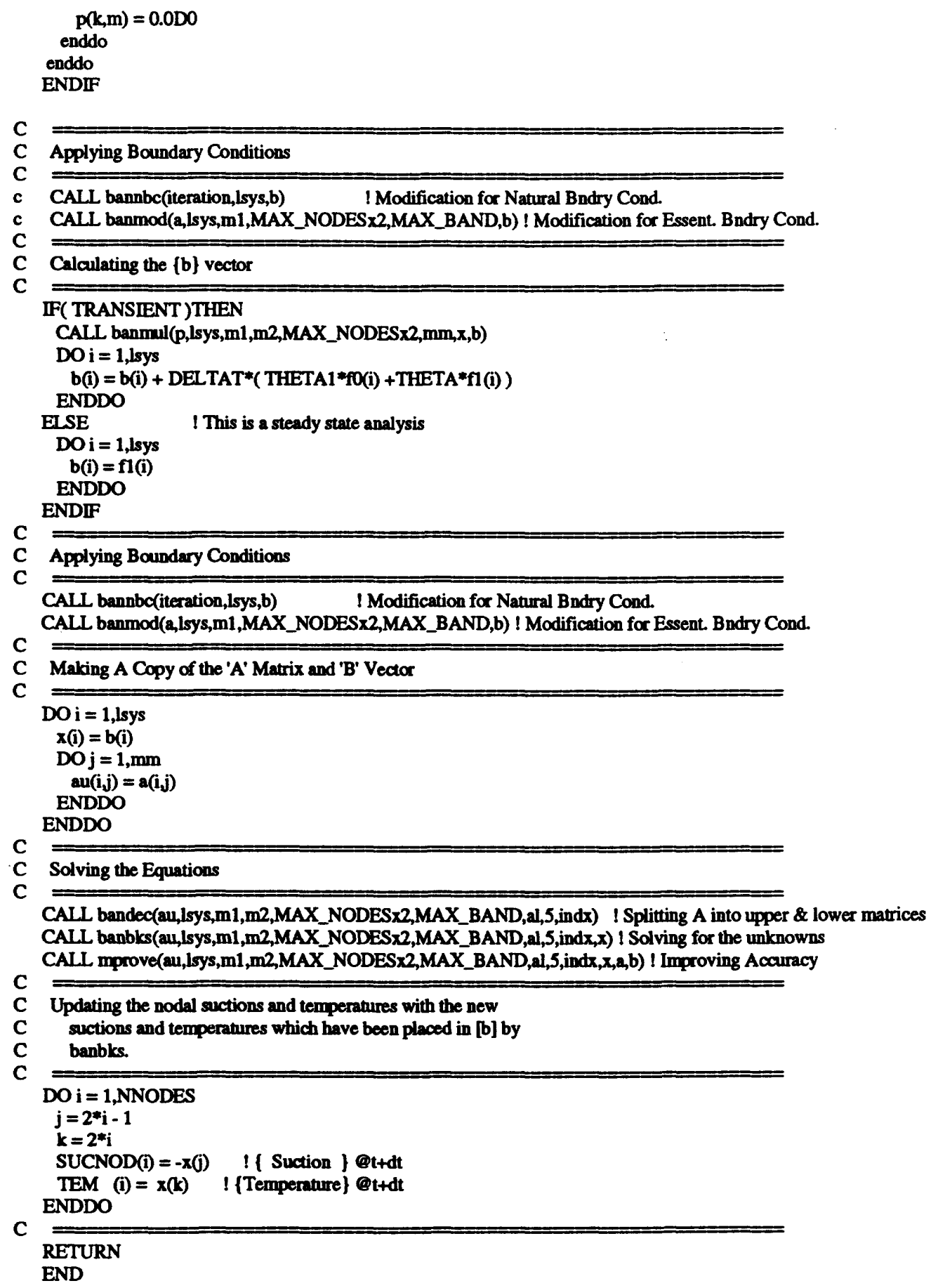

CALL bandec(au,lsys,m1,m2,MAX_NODESx2,MAX_BAND,al,5,indx) ! Splitting A into upper \& lower matrices CALL banbks(au,lsys,m1,m2,MAX_NODESx2,MAX_BAND,al,5,indx,x) I Solving for the unknowns CALL mprove(au,lsys,m1,m2,MAX_NODESx2,MAX_BAND,al,5,indx, a,a,b) ! Improving Accuracy

$c$

C Updating the nodal suctions and temperatures with the new

C suctions and temperatures which have been placed in [b] by

C banbles.

$\mathrm{C}=$ DO $\mathrm{i}=1, \mathrm{NNODES}$

$\mathrm{j}=2 * \mathbf{i}-1$

$\mathbf{k}=2 *_{\mathrm{i}}$

SUCNOD $(i)=-x(j) \quad$ ! ( Suction f @t+dt

TEM $(i)=x(k) \quad !\{$ Temperature $\}$ t $+d t$ ENDDO

C

RETURN

END

C This function is used by the subroutine 'TIERATE' to determine

C if convergence has been achieved.

$\mathrm{C}=$

\section{LOGICAL FUNCTION Convergence()}

$\mathrm{C}=$ IMPLICIT NONE INCLUDE DECLAREFT ! Contains all common block declarations

\begin{tabular}{ll}
\hline INTEGER i,soil & ! Loop Counter \\
LOGICAL converged & ! Logical flag to indicate when system has converged
\end{tabular}



REAL diff
REAL volwc, voluwc
REAL fn_point

C

converged $=$ TRUE

$\mathrm{i}=0$

DO WHIIE (i.LT.NNODES AND. converged )

$\mathrm{i}=\mathrm{i}+\mathbf{1}$

C

C Convergence for suction is based upon a relative convergence
C which is checked at every node.

C

IF(PRESNOD(i).NE.0.0E0)THEN ! Prevent division by zero diff =ABS( SUCNOD(i)-PRESNOD(i) )/PRESNOD(i)

ELSEIF(SUCNOD(i).NE.0.0E0)THEN

diff $=1.0 \mathrm{E} 0$

ENDIF

IF(diff.GT.PUSNORM)THEN

converged $=$ FALSE

IF( STEADYSTATE)THEN

WRITE $(*, 1)$ i,dif* 100.0, SUCNOD(i)

1 FORMATC Node',13;' Change ',F6.2,

1 '\% Suction',G17.4)

ENDIF

ENDIF

C Convergence for temperature is based upon a relative conv.

C which is checked at every node.

C IF(PRETNOD(i).NE.0.0E0)THEN ! Prevent division by zero diff = ABS( TEM(i)-PRETNOD(i))/PRETNOD(i)

ELSEIF(TEM(i).NE.0.0E0)THEN

diff $=1.0 \mathrm{EO}$

ENDIF

IF( diff.GT.PUTNORM)THEN

IF( STEADYSTATE.AND.converged) THEN

WRITE(*,2)i,diff*100.0,TEM(i)

2 FORMATC Node',I3,' Change 'F6.2

1 ENDIF

'\% Temperature',P9.2)

converged $=$ FALSE

ENDIF

ENDDO

Convergence $=$ converged

RETURN

END

\section{subroutine display(ivid,PE,AE,AT,INFIL,Water)}

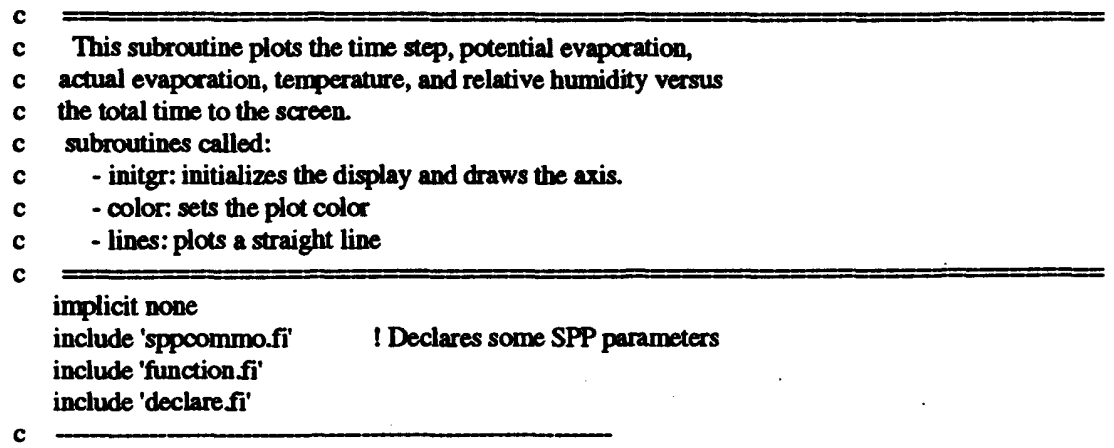




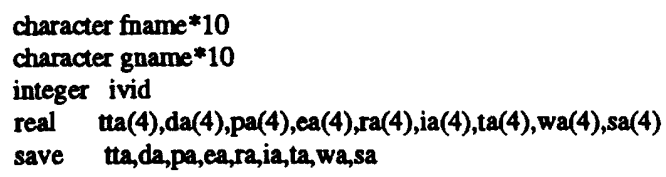


c return

end

\section{SUBROUTINE ELEM1Q1(Element)}

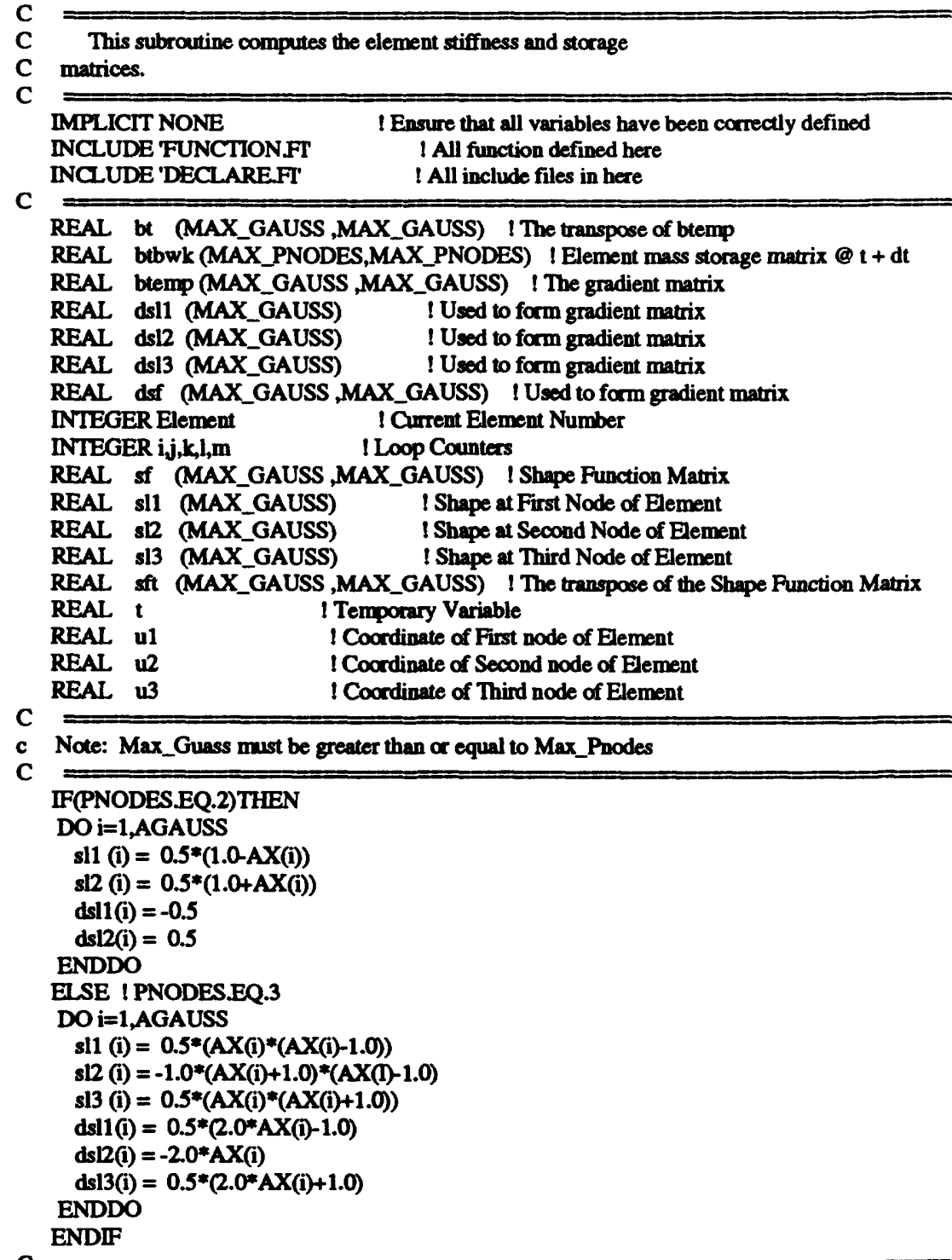

C INITIALIZING THE ELEMENT MATRICES

C

$D O j=1, P N O D E S$

DO $\mathrm{i}=1$,PNODES

$\operatorname{STSW}(i, j)=0.0$ ! Mass moisture storage matrix

STSH $(i, j)=0.0$ ! Mass heat storage matrix

BTBW $(i, j)=0.0$ ! Suction stiffness matrix

btbwk $(i, j)=0.0$ I Load related to gravity $t+d t$

BTBH $(i, j)=0.0$ ! Temperature stiffness matrix

BTBWH $(i, j)=0.0$ ! Suction stiffness matrix associated with Temperature coupling

BTBHW $(i, j)=0.0$ ! Temperature stiffness matrix associated with Suction coupling ENDDO

ENDDO

C STARTING CALCUATIONS IN THE GAUSS LOOP 
C FORMING THE sf AND dsf MATRICES

C

sf $(m, 1)=\operatorname{sil}(\mathrm{m})$

sf $(m, 2)=s 12(m)$

$\operatorname{dsf}(m, 1)=\operatorname{dsl}(m)$

$\operatorname{dsf}(m, 2)=d s 12(m)$

ELSEIF(PNODES.EQ.3) THEN

sf $(m, 1)=s 11(m)$

sf $(m, 2)=s 12(m)$

sf $(m, 3)=s 13(m)$

$\operatorname{dsf}(m, 1)=\operatorname{dsl}(m)$

$\operatorname{dsf}(m, 2)=d s 12(m)$

$\operatorname{dsf}(m, 3)=\operatorname{ds} 13(m)$

ENDIF

C FORMING THE GRADIENT MATRIX

$\mathrm{C} \longrightarrow$

DO $\mathrm{i}=1$, PNODES

$\operatorname{btemp}(m, i)=\operatorname{dsf}(m, i) * D N V J(m)$

ENDDO

C

C FORMING THE TRANSPOSE OF sf,dsf, AND B MATRICES

C

DO $\mathrm{i}=1$, PNODES

sft $(i, m)=s f(m, i) * D E T J(m)$

bt $(\mathrm{i}, \mathrm{m})=$ btemp $(\mathrm{m}, \mathrm{i})$

ENDDO

C FORMING THE PRODUCT MATRICES MULTIPL YING BY GAUSS WTS AND

C SUMMING TO OBTAN THE INTEGRATED BTB AND STS MATRICES

$\mathrm{C}$

DO $\mathrm{i}=1$, PNODES

DO $\mathrm{j}=1$, PNODES

$t=\operatorname{st}(\mathbf{i}, \mathbf{k}) * \operatorname{sf}(\mathbf{k}, \mathbf{j}) * A W(m)$

STSW $(i, j)=t^{*}$ CWMASS $(m)+\operatorname{STSW}(i, j)$

STSH $(i, j)=t^{*}$ CHMASS $(m)+$ STSH $(i, j)$

$t=b t(i, k) * b t e m p(k, j) * A W(m) * D E T J(m)$

BTBW $(i, j)=t^{*}$ CWSTIFF $(m)+$ BTBW $(i, j)$

btbwk $(\mathrm{i}, \mathrm{j})=\mathrm{t}^{*} \mathrm{CWK} \quad(\mathrm{m})+\mathrm{btbwk}(\mathrm{i}, \mathrm{j})$

BTBWH $(i, j)=t^{*}$ CWHSTIFF(m) + BTBWH $(i, j)$

BTBH $(i, j)=t^{*}$ CHSTIFF $(m)+B T B H(i, j)$

BTBHW $(i, j)=t^{*}$ CHWSTIFF(m) + BTBHW $(i, j)$

ENDDO

ENDDO

$\mathbf{k}=\mathbf{k}+\mathbf{1}$

C

300 ENDDO $!$ End of do $m=1$,AGUASS

C Forming the element load vector related to gravity

C DO $1=1$,PNODES

$\operatorname{DSTK}(\mathrm{I})=0.0$

DO $m=1$, PNODES

$\operatorname{DSTK}(\mathrm{l})=\operatorname{DSTK}(\mathrm{l})+\mathrm{btbwk}(\mathrm{l}, \mathrm{m}) * \mathrm{YCORD}(\mathrm{NELCON}(\mathrm{m}$, Element $)) / 100$. ENDDO 


\section{SUBROUTINE FLUX}

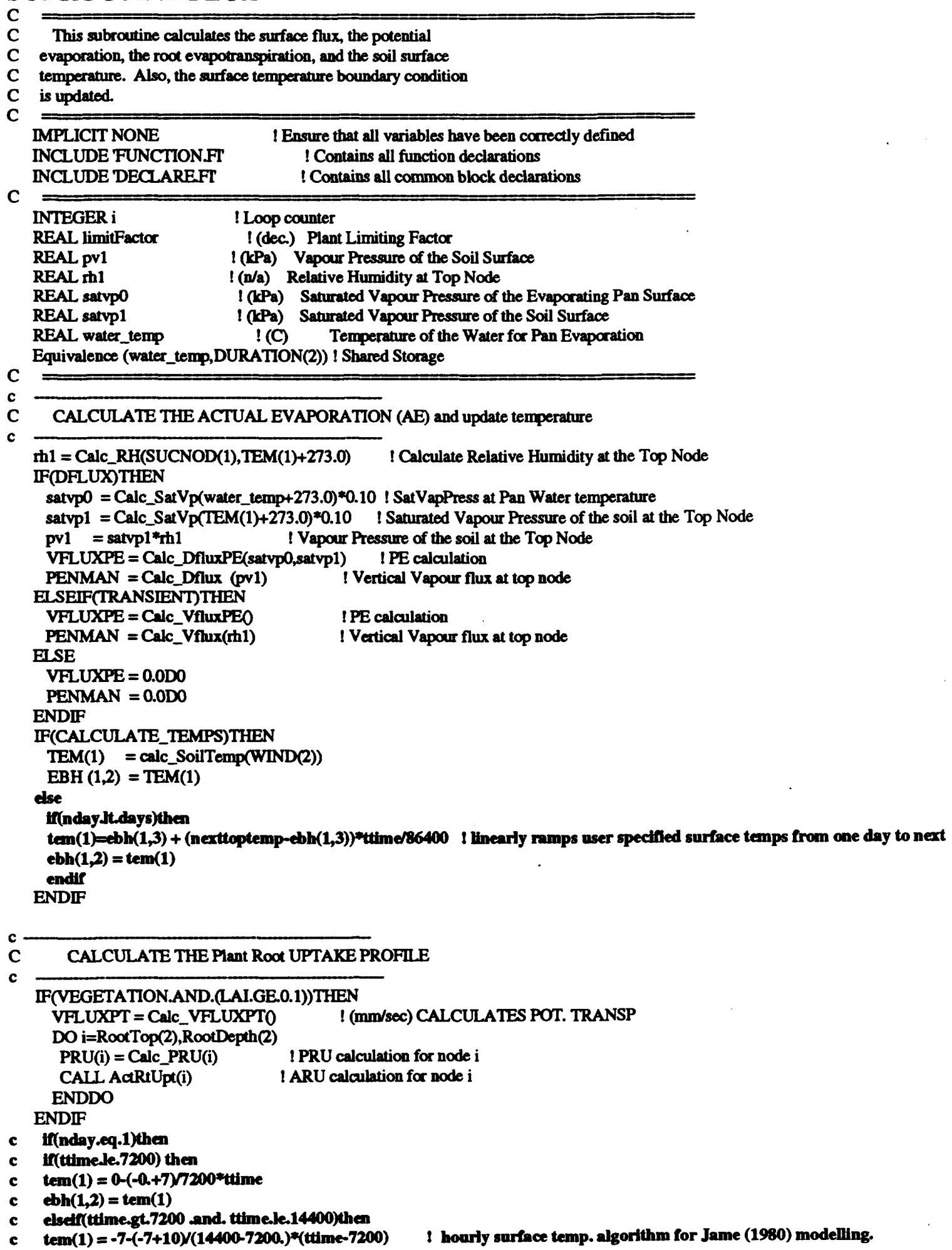


endif

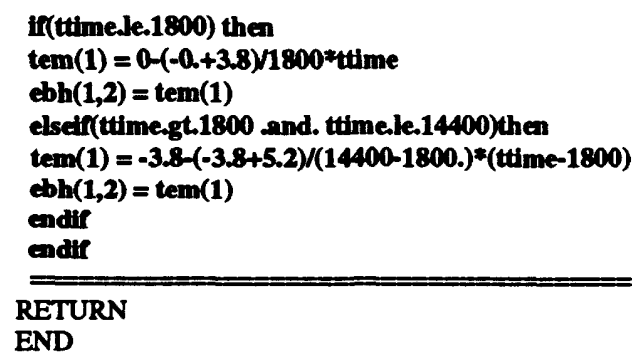

\begin{tabular}{|c|c|}
\hline \multicolumn{2}{|c|}{ SUBROUTINE GAUSS_DATA } \\
\hline $\begin{array}{l}\text { DMPLICIT NONE } \\
\text { INCLUDE 'FUNCTIONFT } \\
\text { INCLUDE 'DECLARE.FT' } \\
\end{array}$ & $\begin{array}{l}\text { ! Ensure that all variables have been correctly defined } \\
\text { I All function defined here } \\
\text { ! All include files in here }\end{array}$ \\
\hline $\begin{array}{l}\text { INTEGER } \\
\text { INTEGER }\end{array}$ & Loop Counter \\
\hline
\end{tabular}

OPEN (UNIT=23,FILE=GaussLcFile,STATUS='OLD) ! Gauss Pt. Locations OPEN (UNIT=24,FILE=GaussWtFile,STATUS='OLD) ! Gauss Pt. Weights DO $\mathrm{i}=1$,AGAUSS I Read Locations \& Weights

$\mathrm{DO} \mathrm{j}=1, \mathrm{i}$

$\operatorname{read}(23, *) \mathbf{A X}(\mathrm{j})$

$\operatorname{read}(24, *) A W(j)$

ENDDO

DO J=i+1,MAX_GAUSS ! Initialize Rest of Array to zero

$\operatorname{AX}(\boldsymbol{J})=0.0$

$\mathrm{AW}(J)=0.0$

ENDDO

ENDDO

CLOSE (UNIT $=23$ )

CLOSE (UNIT $=24$ )

RETURN

END

\section{SUBROUTINE Get_Run_Time}

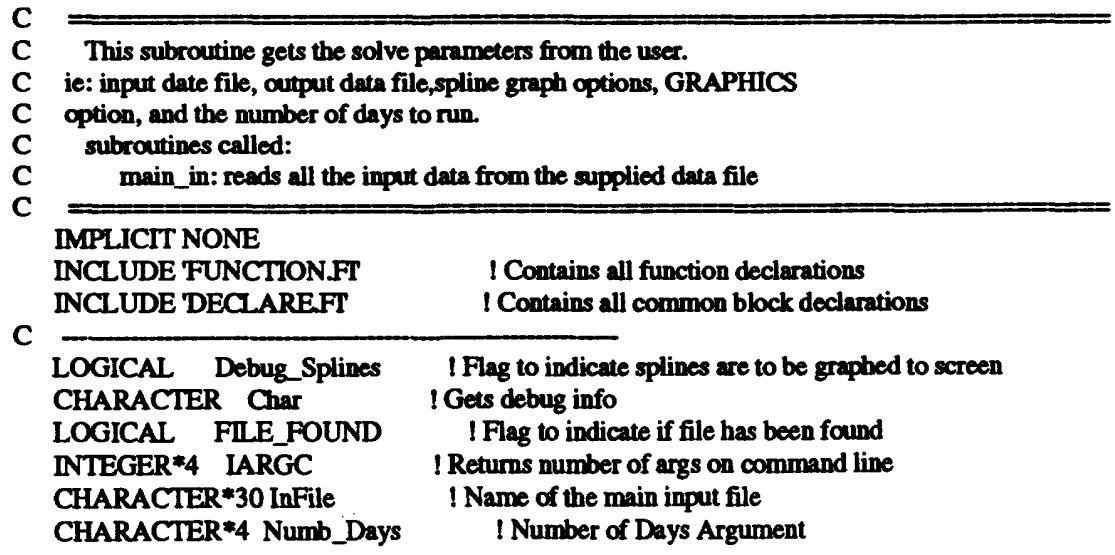




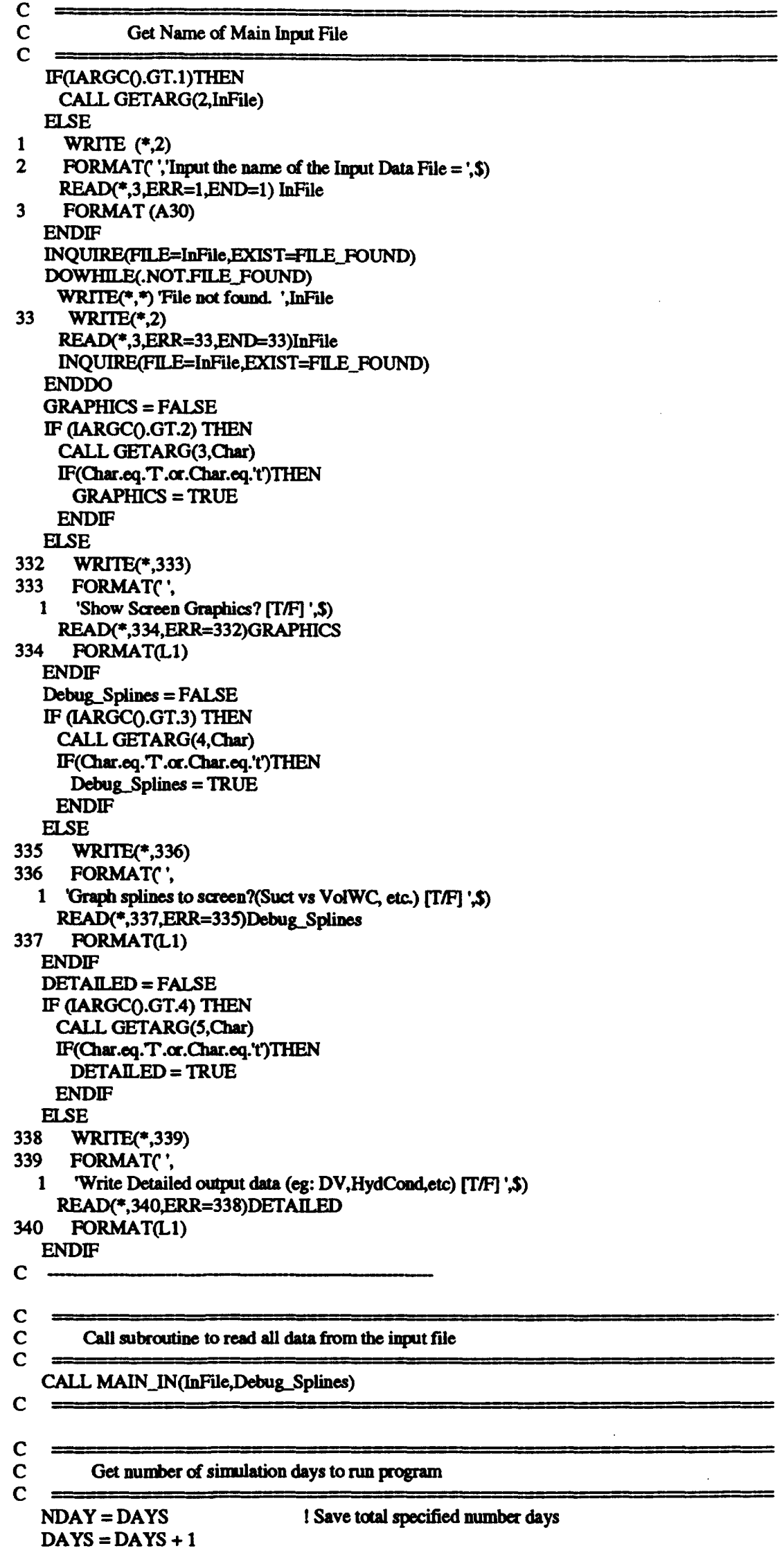




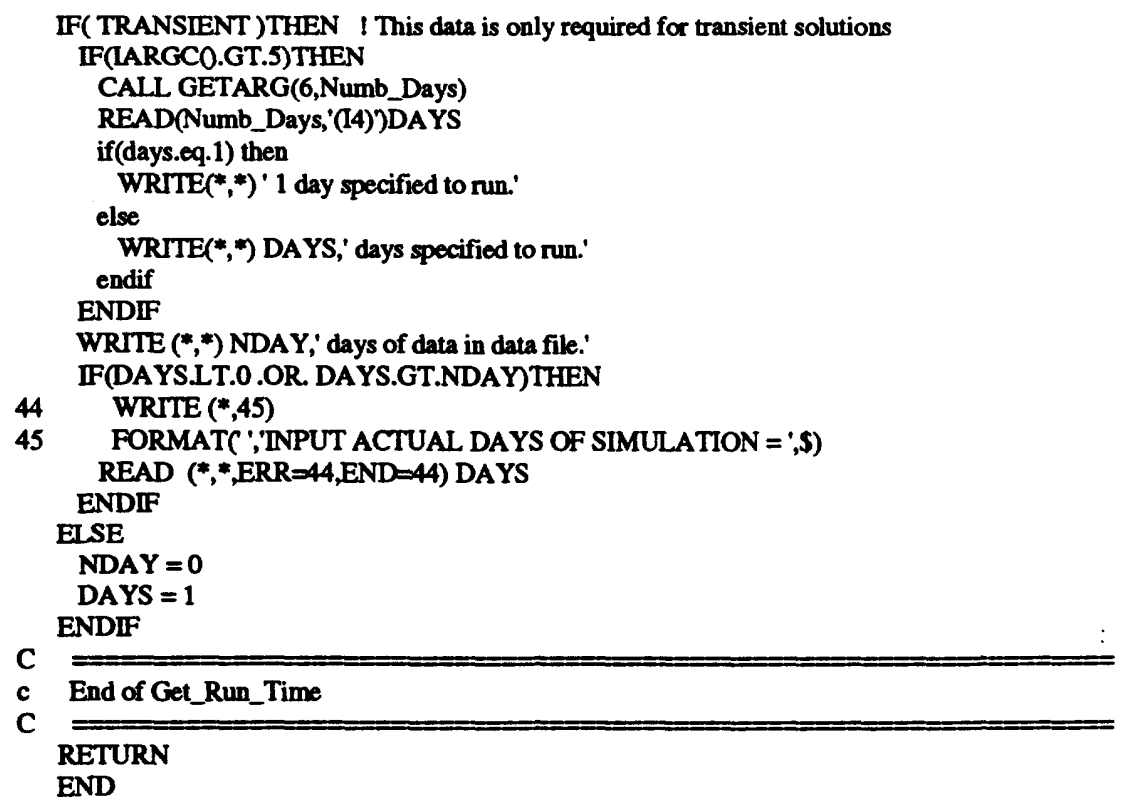

\section{subroutine graph(soil,np,xa,ya,za,yas,zas,X_Label,Y_Label,type)}

c This subroutine takes spline data and generates points from the

c spline. The splines is then printed to the screen.

c include 'sppcommo.fi' ! Declares some SPP parameters include 'constant.fi' ! declares array const.

c

integer points

parameter (points $\mathbf{1 0 0 0}$ )

character X_Label*(*) IX Axis Label

character Y_Label*(*) IY Axis Label

character fname*10

integer $i j$ ! loop counters

integer soil !Current layer

integer np(max_types) ! Number of points per layer

integer type IType of Plot Required

real $x$ (points +2) IX Coordinate for cutput points from spline data

real $x a\left(\max \_\right.$points,max_types) $1 \mathrm{X}$ cocrdinates of spline data

real y(points+2) I Y Coordinate for output points from spline data

real ya(max_points,max_types) IY cocrdinates of spline data

real yas(max_points,max_types) ! Smoothed Y Coordinate for output points from spline data

real ys(points +2$) \quad$ I Y Coordinate for output points from spline data

real z(points+2) ! Slope of spline at different points

real za(max_points,max_types) ! Curvative of spline data

real zas(max_points,max_types) ! Smoothed Slope of spline at different points

real zs(points+2) ISlope of spline at different points

real Fn Point I Fortran spline function which returns a spline value

real Fn_Slope ! Fortran spline function which returns the slope of a spline

c $=16$ ! MCA Graphics Display

ifore $=15 \quad !$ White Foreground Color

iback = 16 ! Blue Background Color

nprin $=8$

mode $=5$

isave $=-1$

fname='memory'

call vsinit(mon,10.,8.,isave,fname,iunitv,ivid,ifore,iback,iunitm)

call linwid $(0,01)$

call setasp (1.0) 

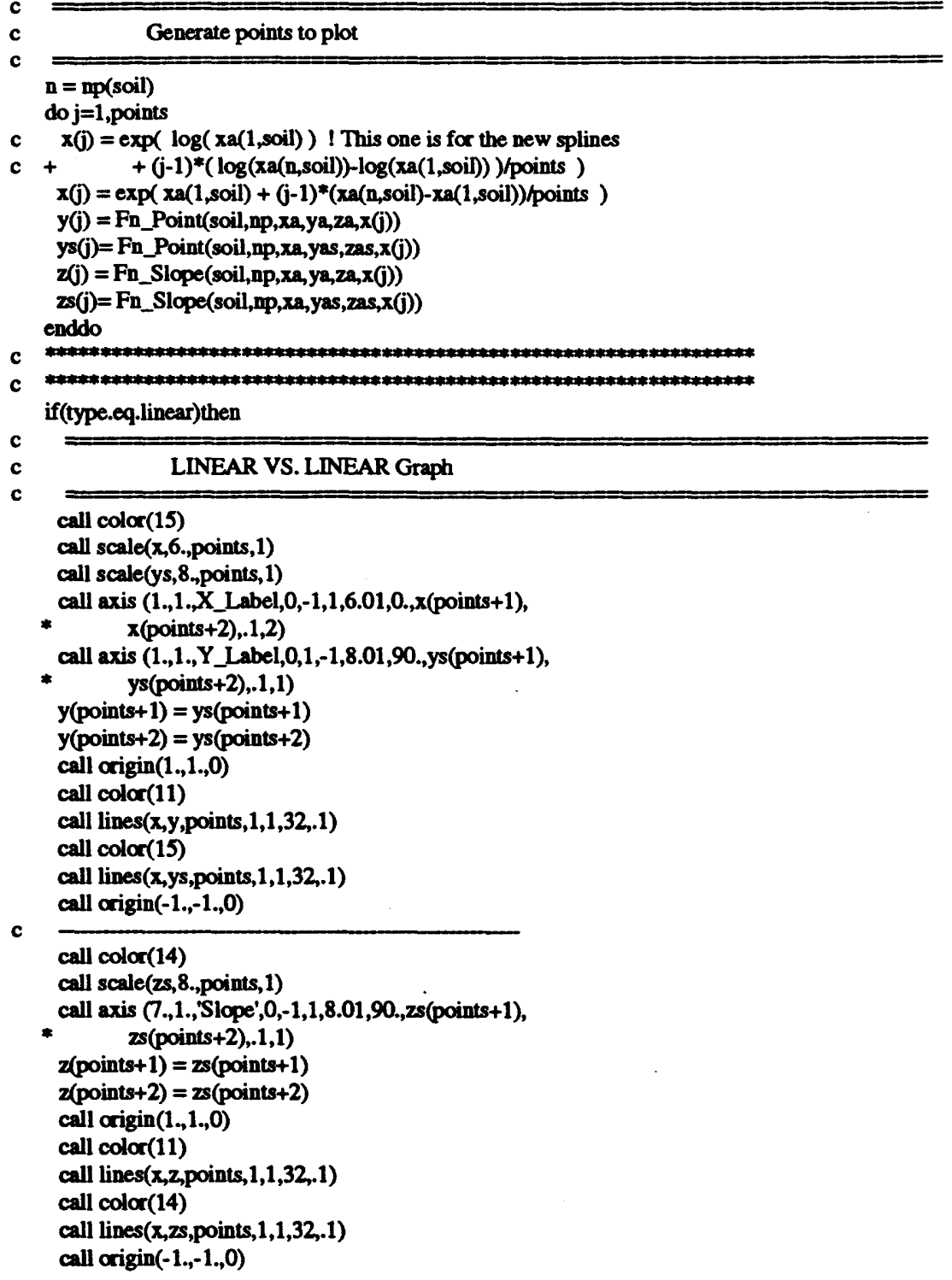

c Plot user supplied data points

c call color(11)

do $i=1, n$

$x(i)=\exp (x a(1$, soil) $)$

$y(i)=\exp (y a(i, s o i l))$

enddo

$x(n+1)=x($ points +1$)$

$x(n+2)=x$ (points +2$)$

$y(n+1)=y($ points +1$)$

$y(n+2)=y($ points +2$)$

call arigin $(1 ., 1 ., 0)$

call lines $\left(\mathrm{x}, \mathrm{y}, \mathrm{n}, 1,-1, \mathrm{ichar}\left(\mathrm{O}^{\prime}\right), \mathbf{1}\right)$

call origin $(-1,-1,0)$

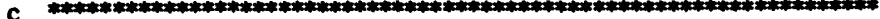

C

else if(type.eq.semi_log)then

c LOG VS. LINEAR GRAPH

c $=$

call color(15)

call lgscal (x,6.,points, 1 )

call scale (ys,8.,points,1) 
call lgaxis(1.,1.,X_Label,0,-1,1,6.01,0.,x(points+1),

$$
\text { * } \quad x \text { (points+2),.1) }
$$

call axis (1.,1.,Y_Label,0,1,-1,8.01,90.,ys(points+1),

*ys(points+2),.1,3)

$y($ points +1$)=y s($ points +1$)$

$y($ points +2$)=y s$ (points +2$)$

call origin (1.,1.,0)

call color(11)

call lgline( $x, y$, points, 1, points/10,32,-1,.1)

call color (15)

call lgline( $x, y s, p o i n t s, 1$, points/10,32,-1,.1)

call origin $(-1 .,-1 ., 0)$

c

call color(14)

call scale (zs,8.,points,1)

call axis (7.,1.,'Slope',0,-1,1,8.01,90.,zs(points+1),

* $\quad 2 s$ (points+2),.1,3)

$z($ points +1$)=z s($ points +1$)$

$z$ (points +2$)=z s$ (points +2 )

call origin(1.,1.,0)

call color(11)

call Igline( $x, z$ points, 1, points/10,32,-1,1)

call color(14)

call Igline(x,2s,points, 1,points/10,32,-1,.1)

call origin $(-1,,-1,0)$

c Plot user supplied data points

call color(11)

do $i=1, n$

$x(i)=\exp (x a(i, s o i l))$

$y(i)=\exp (y a(1$, soil $))$

enddo

$x(n+1)=x$ (points +1$)$

$x(n+2)=x$ (points +2$)$

$y(n+1)=y$ (points +1$)$

$y(n+2)=y($ points +2$)$

call origin $(1 ., 1 ., 0)$

call lgline( $x, y, n, 1,-1$, ichar(O), $-1, .1)$

call origin $(-1,,-1 ., 0)$

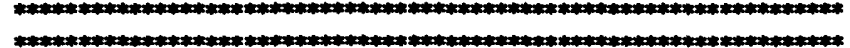

else if(type.eq.logarithmic)then

c

c

call color(15)

call lgscal (x,6.,points, 1 )

call lgscal(ys,8.,points,1)

call lgaxis(1.,1.,X_Label, 0,-1,1,6.01,0.,x(points+1),

* $\quad x$ (points+2), 1 )

call lgaxis(1.,1.,Y_Label,0,1,-1,8.01,90.,ys(points+1),

* ys(points+2),.1)

call origin $(1,, 1 ., 0)$

$y($ points +1$)=y s($ points +1$)$

$y($ points +2$)=y s($ points +2$)$

call color(11)

call Igline( $x, y$, points, 1, points/10,32,0,.1)

call color (15)

call lgline( $x, y s$, points, 1, points $/ 10,32,0, .1)$

call origin(-1.,-1.,0)

c

call color(14)

call scale (2s,8.,points,1)

call axis (7.,1.,'Slope',0,-1,1,8.01,90.,zs(points+1),

* zs(points+2),.1,3)

$z$ (points +1$)=z s$ (points +1$)$

$z$ (points +2$)=z s$ (points +2$)$

call origin(1.,1.,0)

call color(11)

call lgline(x,z,points, 1,points/10,32,-1,.1) 
call color(14)

call lgline(x,2s,points,1,points/10,32,-1,.1)

call origin $(-1,-1 ., 0)$

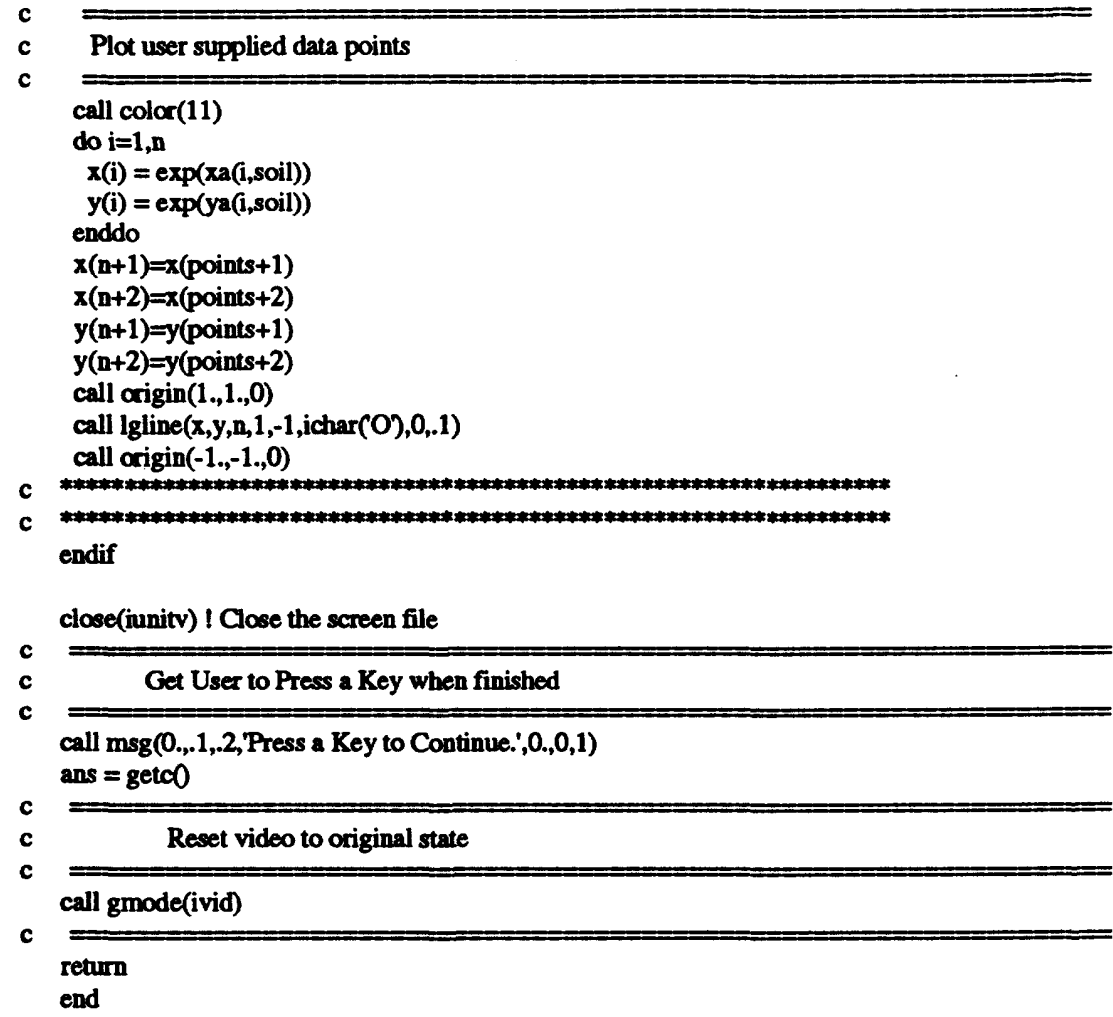

C

\section{SUBROUTINE DICE(oldtemp,newtemp,oldsuc,newsuc)}

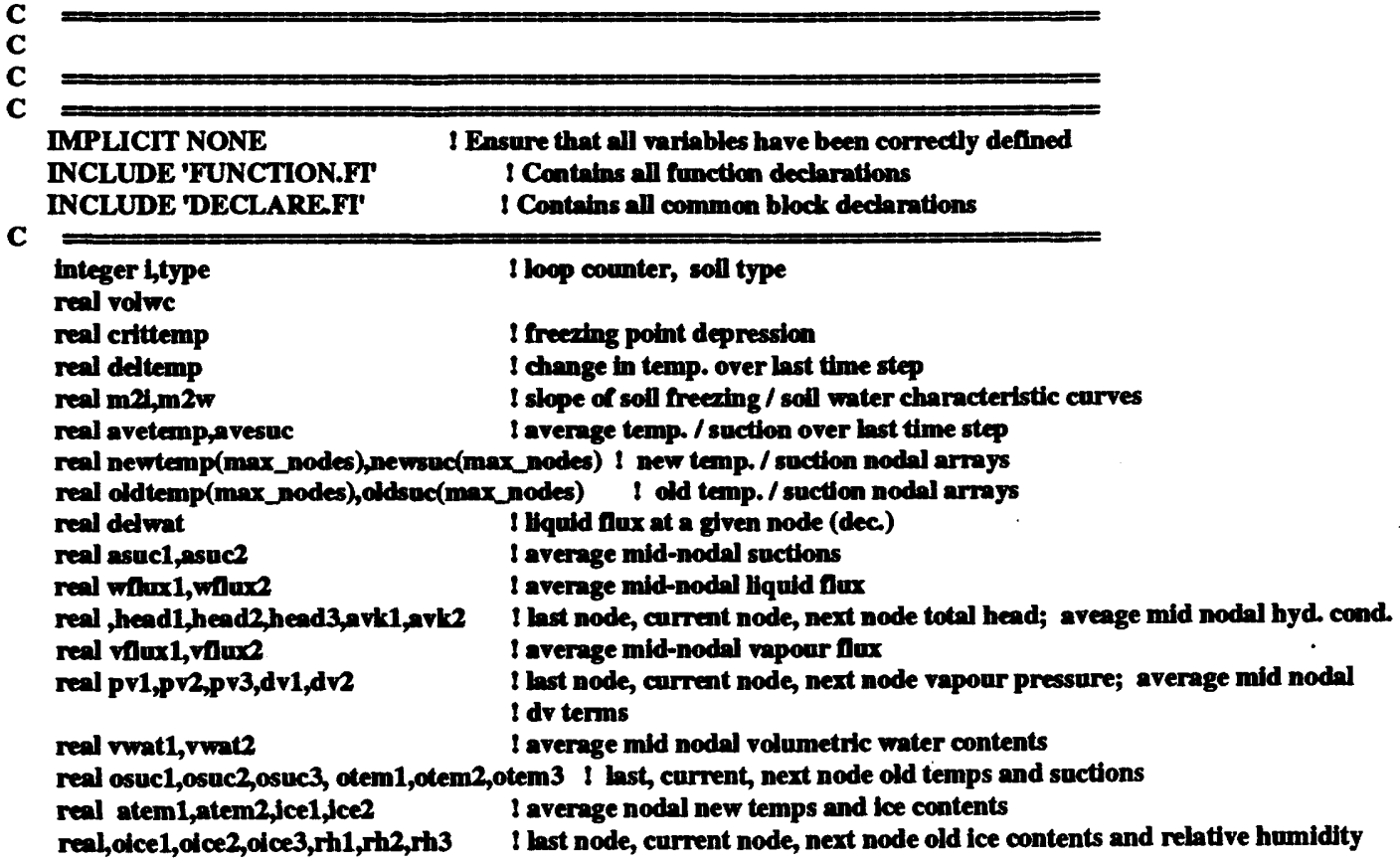

do $i=1$,nnodes 
C Calculate the critical temperature for freesing

type=solltype(i)

volwc=calc_volwo(type,oldsud(i)) ! based on start of time step suction crittemp $=$-in_point(type,points7,xvoluwc,xtem,splins17,volwc)

if(nodvolice(i).gt.0.) crittemp=oldtemp(i)

C Calculate average temp. and if freeding or thawing occured

C if(newtemp(i).Je.crittemp.and.oldtemp(i).tt.-0.05) then 1 freeaing avetemp $=$ (newtemp $(1)+$ erittemp)/2. deltemp=newtemp(i)-crittemp

elseif(newtemp(i)gtcrittempandnewtemp(i).tt.-0.05 I thawing. Note: $-0.05^{\circ} \mathrm{C}$ chosen to

1 and.nodvolice(i) gt.0.)then I prevent errors using SFC near $0^{\circ} \mathrm{C}$.

avetemp $=($ newtemp $(1)+c r i t t e m p) / 2$.

deltemp=newtemp (i)-crittemp

elseif(newtemp(i).ge-0.05.andnodvolice(i).gt.0.)then I thawing

avetemp $=(-0.05+$ erfttemp $) / 2$.

in(avetemp.gt.0) avetemp $=$-avetemp

clse

deltemp $=$ abs $(-0.05$-crittemp)

avetemp $=99$. I no freexing or thawing happening at this node

endir

C

C Calculate liquid fux over previous time step

if(avetemp.nes9) then

ir(i.eq.1) then

osue1 $=0$ ldsuc(i)

otem1=oldtemp(i)+273.16

oice1 $=$ nodvolice(i)

clse

osuc1 $=0$ idsuc( $(1-1)$

otem1=oldtemp(1-1)+273.16

olce 1=nodvolice(1-1)

endif

osuc2=oldsuc(i)

otem2=oldtemp $(\mathbf{i})+273.16$

oice2=nodvolice(i)

Ir(Leq nnodes) then

osuc3=oldsuc(i)

otem3=oldtemp(i)+273.16

oice $3=$ nodvolice(i)

clse

osuc3=oldsuc $(i+1)$

otem3=oldtemp(1+1)+273.16

oices=nodvolice (i+1)

endir

asuc1 $=($ osuc1+osuc2 $) / 2$

asuc $2=(0 \operatorname{coc} 2+0 \operatorname{coc} 3) / 2$.

atem1 $1=(\operatorname{otem} 1+\operatorname{otem} 2) / 2$

atem $2=(\operatorname{otem} 2+\operatorname{otem} 3) / 2$

ice1=(oice1+oice2) 2 .

ice $2=(0 i c e 2+o i c e 3) / 2$.

avk1=calc_k(type,asuc1,ice1)

avk2=calc_k(type,asuc 2,jice2)

dv1=calc_vapour_din(atem1, vwat1,ice1,pord(type))

dv2=calc_vapour_difr(atem2, vwat2,jce2,pord(type))

ir(i.eq nnodes) then 
head1=ycord(1-1)/100.-0suc1/grav
head2=ycord(1)/100.-0suc2/grav

rh1=calc_rh(oldsuc(1-1),oldtemp $(j-1)+273.16)$

rh2=calc_rh(oldsuc(i),oldtemp $(i)+273.16)$

pv1=calc_eatvp(oldtemp $(1-1)+273.16) * \mathrm{rh1} * 0.1$

pv2-calc_satvp(oldtemp $(1)+273.16) * \mathrm{rh2} * 0.1$

wior $2=a v k 2 *($ head 2 head1 $) /(y \operatorname{cord}(i)-y \operatorname{cond}(1-1)) * 100$.

vilux $2=d$ 2*(pv2-pv1)/(ycond(i)-ycond(i-1))*100

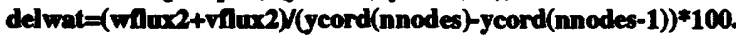

elseir(teq.1) then

head 1 =ycord(i)/100.-0suc2/grav

head2 $=$ ycord $(1+1) / 100$-ocuc3/grav

rhl=calc_Ih(oldsud(i),oldtemp(i)+273.16)

th2=calc_rh(oldsuc(i+1), oldtemp $(i+1)+273.16)$

pv1=ealc_entvp(oldtemp $(1)+273.16) *$ rh1 $* 0.1$

pr2=calc_satvp(oldtemp $(1+1)+273.16) * \mathrm{rh2} 20.1$

wilux 1=-avk1*(head2-head1)/(ycord(i)-ycord(i+1))*100.

vilur1 $=-d v 1 *(p v 2-p v 1) /(\operatorname{reord}(i)-y \operatorname{cord}(1+1)) * 100$

delwat $=($ wox $1+v i$ wax $) /(y \operatorname{cond}(2)-y \operatorname{cond}(1))^{* 100}$.

che

head1=ycord(1-1)/100,-osuc1/grev

head $\Rightarrow$ ycord $(1+1) / 100$-osuck/grav

head2-ycord(i)/100.-0suc2/grav

rh1 =calc_rh(oldsuc(l-1), oldtemp(1-1)+273.16)

rh2=calc_holdsuc(i), cldtemp(i)+273.16)

rh3=calc_h $h($ oldsuc $(t+1)$, oldtemp $(i+1)+273.16)$

pv1=calc_satvp(oldtemp(1-1)+273.16)*rh1*0.1

pr2=cak_sotvp(oldtemp(i)+273.16)*rh2*0.1

pv3=calc_entvp(oldtermp $(1+1)+273.16) *$ rh3*0.1

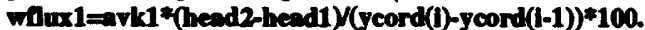

wilur 2-avl 2* (head3-bead2) $($ ycord $(1+1)-y \operatorname{cord}(1)) * 100$.

vilux1 $=d v 1 *(p v 2-p v 1) /($ cond $(1)-y \operatorname{cond}(1-1) * 100$

vilux?=dv2*(pr3-pr2)/(reord(1+1)-ycond(i))*100

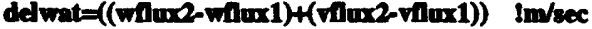

1 Kroord(1+1)-ycond(1-1))*200. imatm /sec.

endir

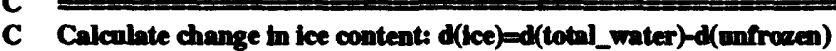

C

m21=-in_slope(type,points\&, ytem,yvoluwe,splinsis,abs(avetemp))

delice $(i)=($ delwat* deltat-m2i*deltemp)/rholce

else

delice $(i)=0$.

endil lavetemp.ne.99

If(modvolice(i)+delice(i)de.0.) then I make sure node ice content not negative

delice(i)=-nodvolice(i)

endif

if(newtemp(i).ge-0.05) then I make sure no loe above freesing

delice(i)=-nodvolice(i)

endif

endio

return

end 


\section{subroutine init_graph(ivid,tta,da,pa,ea,ra,ia,ta,wa,sa)}

c This routine initializes the display for the display subroutine

include 'SPPCOMMO.fi'
INCLUDE FUNCTION.FT

INCLUDE 'DECLARE.FT I Contains all declarations

c

character fname $* 10$

character gname*10

character message $* 20$

integer ivid

real tta(4), da(4),pa(4),ea(4),ra(4),ia(4),ta(4),wa(4),sa(4)

c

mon $=16 \quad 1$ MCA Graphics Display

ifore $=15$ ! White Foreground Color

iback = 16 ! Blue Background Color

uprin $=8$

mode $=5$

isave $=-1$

finame=-memary'

call vsinit(mon,10.,8.,isave,fname,iunitv,ivid,ifore,iback,iunitm)

call linwid( 0,01$)$

call setasp(1.0)

Initialize Deltat Scales

call color(15)

da(1) = MIN DELTAT

$\operatorname{da}(2)=$ MAX_DELTAT

$\tan (1)=0.0$

$\operatorname{ta}(2)=24.0$

call scale(tta,5.,2,1)

call lgscal(da,2,2,1)

call axis (1.2,1.,'Time (hrs)',10,-1,1,5.21,0.0,

$1 \operatorname{ta}(3), \operatorname{ta}(4), 1,1)$

call Igaxis(1.2,7., 'Time Step (seconds)'

$1 \quad 19,1,-1,2.01,90 ., \mathrm{da}(3), \mathrm{da}(4), .1)$

c Initialize Suction Scale

c call $\operatorname{color}(12)$

$\operatorname{sa}(1)=0.1$

$\operatorname{sa}(2)=1000000.0$

$\operatorname{ta}(1)=0.0$

$\operatorname{tta}(2)=24.0$

call Igscal $(s a, 5.6,2,1)$

call Igaxis(1.2,1.,'Surface Suction (kPa)',

$121,1,-1,5.61,90 ., \operatorname{sa}(3), \operatorname{sa}(4), .1)$

c Initialize PE \& AE Scales

c $=-14.0$

$\operatorname{pa}(1)=-14.0$

$\operatorname{pa}(2)=+22.0$

call color(11)

call scale(pa,8.,2,1)

do $\mathrm{i}=1,4$

ea(i) $=$ pa(i)

$\operatorname{ra}(i)=\operatorname{pa}(i)$

ia(i) $=\operatorname{pa}(i)$

enddo

call axis (.5,1.,'Surface Flux (mm/day)',

$121,1,-1,8.01,90 ., \mathrm{pa}(3), \mathrm{pa}(4), 1,1)$

c Initialize Temperature Scale

c $\mathrm{ta}(1)=10.0$ 
$\operatorname{ta}(2)=40.0$

call color(14)

call scale $($ ta, 8.,2,1)

call axis (6.5,1.,'Surface Temperature (Celcius)',

$129,-1,1,8.01,90, \operatorname{ta}(3), \operatorname{ta}(4), .1,1)$

c Initialize Water Balance Scale

$w a(1)=-1.5$

$\mathrm{wa}(2)=+1.5$

call color(4)

call scale(wa, 8.,2,1)

call axis (7.2,1.,'Water Balance (mm)',

$1 \quad 18,-1,1,8.01,90$.,wa(3),wa(4),.1,1)

call color(15)

write(message,'(A16,14)) 'Running Day ',NDAY

call msg(0.,.1,2,message, $0 ., 0,1)$

call origin(1.2,1.,0)

return

end

\section{SUBROUTINE ITERATE}

C This subroutine performs an iterative loop until the solution

C has converged or the maximum number of iterations has been performed

C The element, global, system stiffness and storage storage matrices

C are developed as well as the system load vectors. The coupled system

C of simultaneous equations is solved to determine the new nodal suctions

C and temperatures.

Subroutines called:

- CNICOL isolver for a transient analysis

- SOLVE :solver for a steady state analysis

- ELEM1Q1 sets up the elemental stiffness and mass matrices.

-FLUX :calculates the evaporative flux

- JSHAPE :the shape function, used to interpolate properties to gauss pts.

- RELAXATION:implements a relaxation scheme for the iterative loop.

- DICE :calculates the nodal change in ice content over previous time step

\section{IMPLICIT NONE}

INCLUDE 'DECLARE.FT

INCLUDE FUNCTION.FT

REAL Calc_gFlux

! All include files in here

! All functions defined here

! Function to calculate fluxes at element boundaries

REAL avesuc

LOGICAL converged

REAL dv

I Suction at Gauss Point at the half time step

! Temparary Storage for Water Contents/Suctions

! Logical flag to indicate when system has converged

gat ga NODES ,MAX NODES ) ! Global Heat Stiffness Matrix

REAL gbtbhw (MAX_NODES ,MAX_NODES ) ! Global Heat Coupled to Moisture Stiffness Matrix

REAL gbtbw (MAX NODES ,MAX_NODES) ! Global Moisture Stiffness Matrix

REAL gbtbwh (MAX_NODES ,MAX_NODES) ! Global Moisture Coupled to Heat Stiffness Matrix

REAL gcord (MAX_GAUSS) I Gaussian Coordinates

REAL glh (MAX_NODES) ! Global Heat Load Vector

REAL glw (MAX_NODES) ! Global Moisture Loed Vector @ t + dt

REAL gstsh (MAX_NODES,MAX_NODES ) ! Global Heat Mass Storage Matrix

REAL gstsw (MAX_NODES ,MAX_NODES ) ! Global Moisture Mass Storage Matrix

REAL gtemp (MAX_GAUSS) I (K) Temperature at Gauss Pts

INTEGER $i, j, k, 1, m$

REAL lastsuc

REAL lastpv

INTEGER type

LOGICAL maxd out

INTEGER iteration

REAL pv

Loop counters

I Suction at last Gauss Point at the half time step

! Vapour Pressure at Gauss Pts.

1 Current Layer

1 Logical switch set when IIER $=$ MXITER

1 Current iteration number

REAL rd1,rd2

! Vapour Pressure at Gauss Pts.

! Multiplier for Isothermal \& Thermal Vapour 


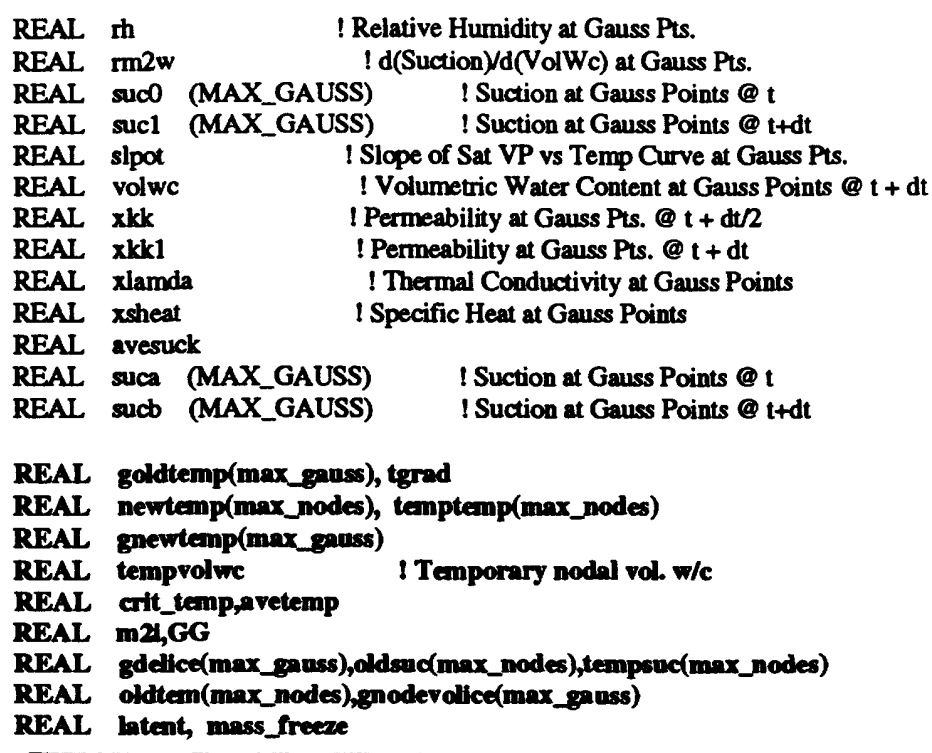

REAL goldtemp(max_gauss), tgrad

REAL newtemp(max_nodes), temptemp(max_nodes)

REAL gnewtemp(max gauss)

REAL temprolwe

I Temporary nodal vol. w/c

REAL crit_temp,avetemp

REAL m24,GG

REAL gdelice(max_ganss),oldsuo(max_nodes),tempsuc(max_nodes)

REAL oldtem(max_nodes), genodevolice(max_gauss)

REAL batent, mass_freeze

C

open(unit=27,file='test.dat',status='new')

iteration $=-1$

converged $=$ FALSE

maxd_out = FALSE
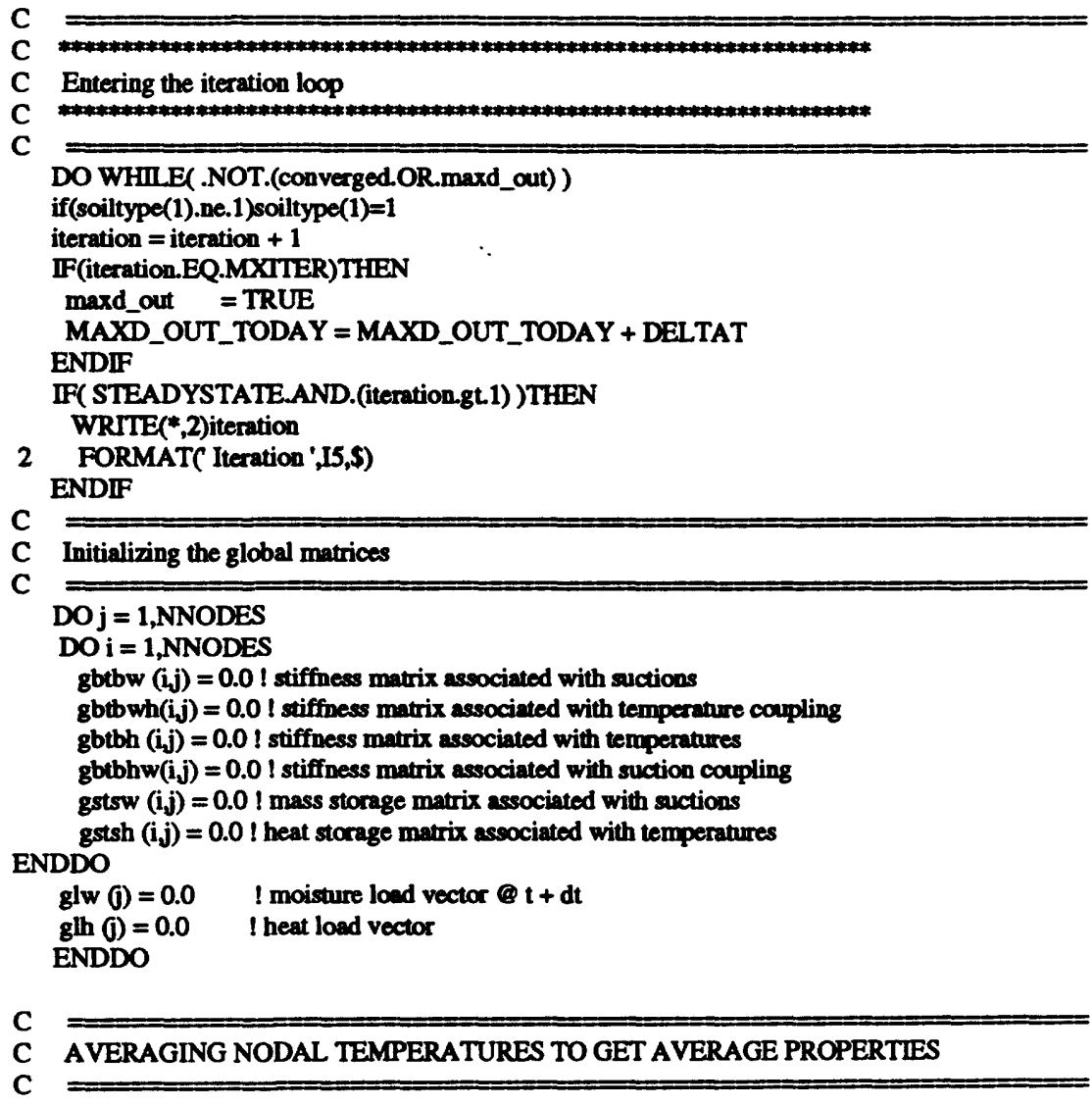

do $i=1$, nnodes

$\mathbf{j}=\mathbf{i}+$ nnodes

OLDTEM(i)=PHIA $(j) \quad$ l oldtemp=newtemp on fird iterntion

$\operatorname{OLDSUC}(\mathrm{l})=\operatorname{PHIA}(\mathrm{l})$

newtemp(i)=tem(I) I new temp is needed for freese analysis

$\operatorname{tem}(\mathrm{i})=(\operatorname{tem}(\mathrm{i})+$ phia $(\mathrm{j})) / 2 . \quad$ tem(i) is now average over dt 


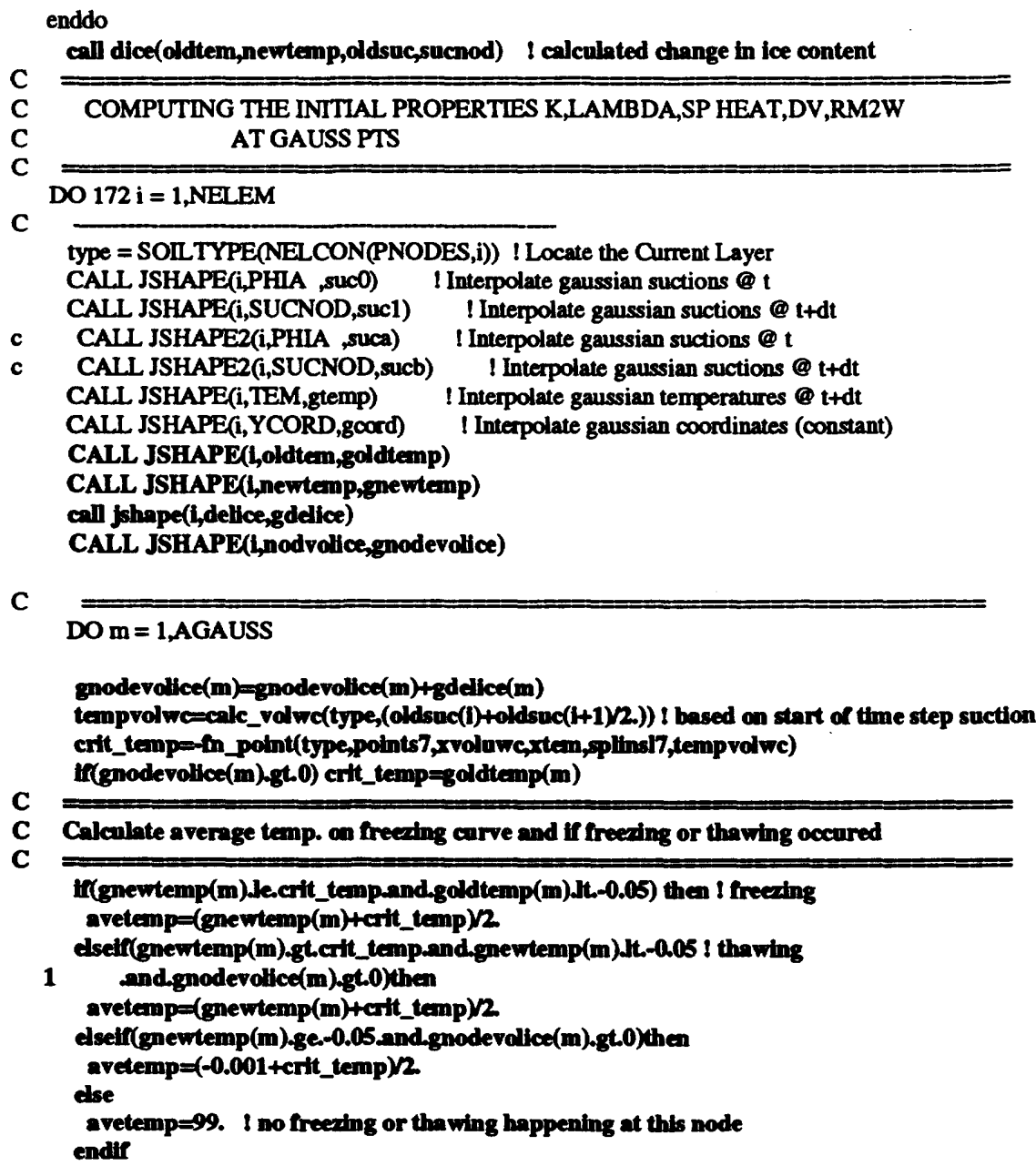

IF (gtemp(m).LT.273.16) slpot=0.05475*exp(0.0802*(gtemp(m)-273.16) I Slope if negative temps. 


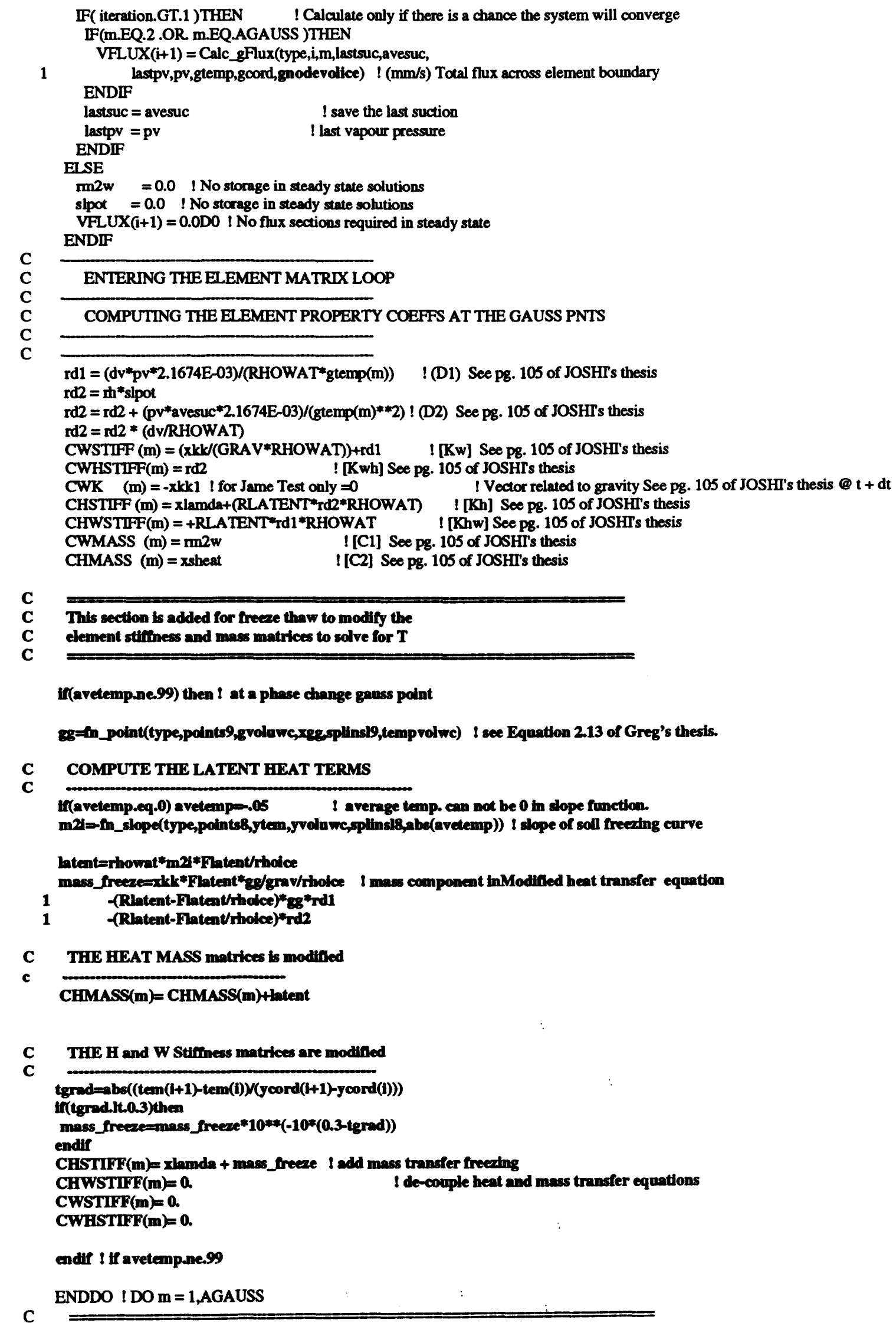


C Forming the element stiffness (BTB) and mass storage (STS) matices

C

CALL EIEM1Q1(i)

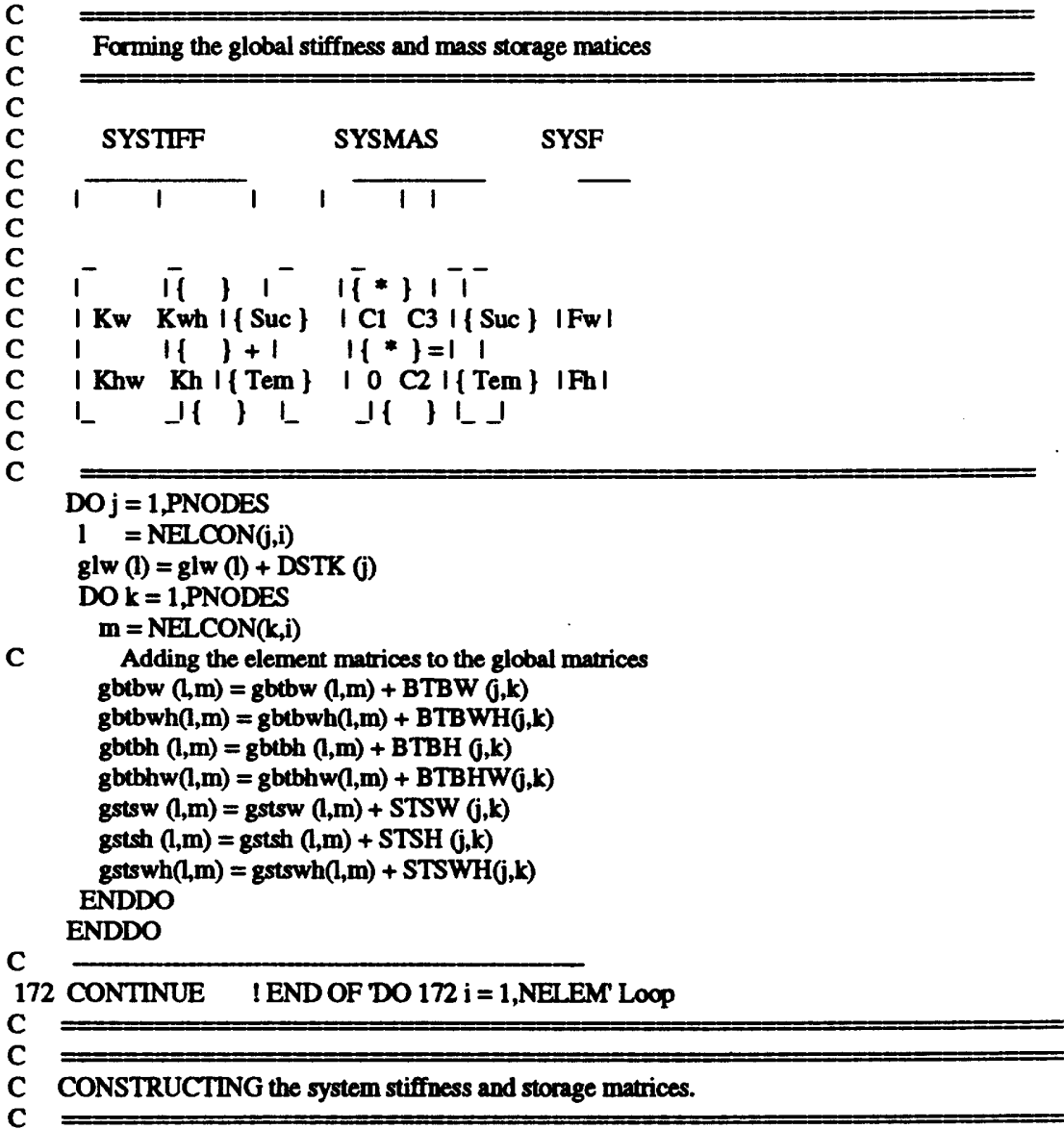

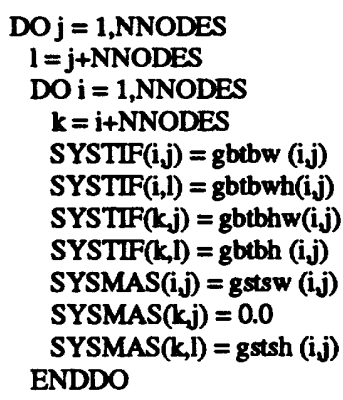

$\operatorname{SYSF}(j)=\operatorname{glw}(j)$

$\operatorname{SYSF}(\mathbf{l})=$ glh $(j)$

ENDDO

C

CALL CNICOL(iteration) ISolve for Nodal Suctions and Temperatures

C

C Modify suctions based on newly solved temperature below freeding when loe has formed.

CALL REVERSE_SPLINES $!$ Reverse the splines order so the it is in ascending suction order DO $\mathrm{i=1,MAX}$ TYPES

CALL WtSplin2(1,POINTS1,XVOLWC,XSUC,SPLINSL1)

ENDDO

DO $\mathrm{i}=1$, NNODES 


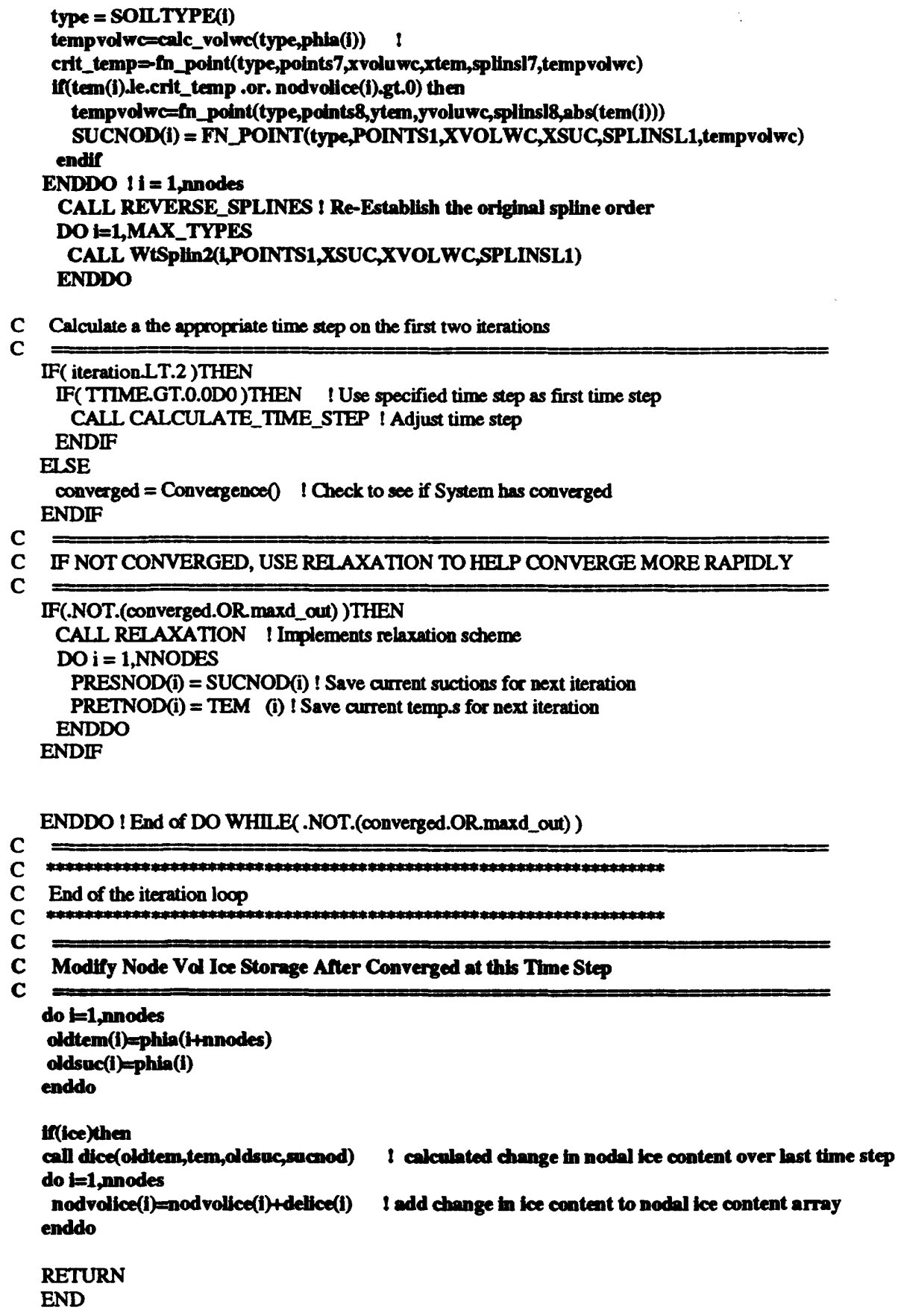
C
SUBROUTINE JSHAPE(Element,NP,GP)
C This subroutine determines the shape functions for two or three
C noded elements (see page 103 JOSHI thesis)
C
IMPL ICIT NONE
INCLUDE 'FUNCTION.FT
! Ensure that all variables have been correctly defined
INCLUDE DECLARE.FT
I Contains all function declarations
I Contains all common block declarations




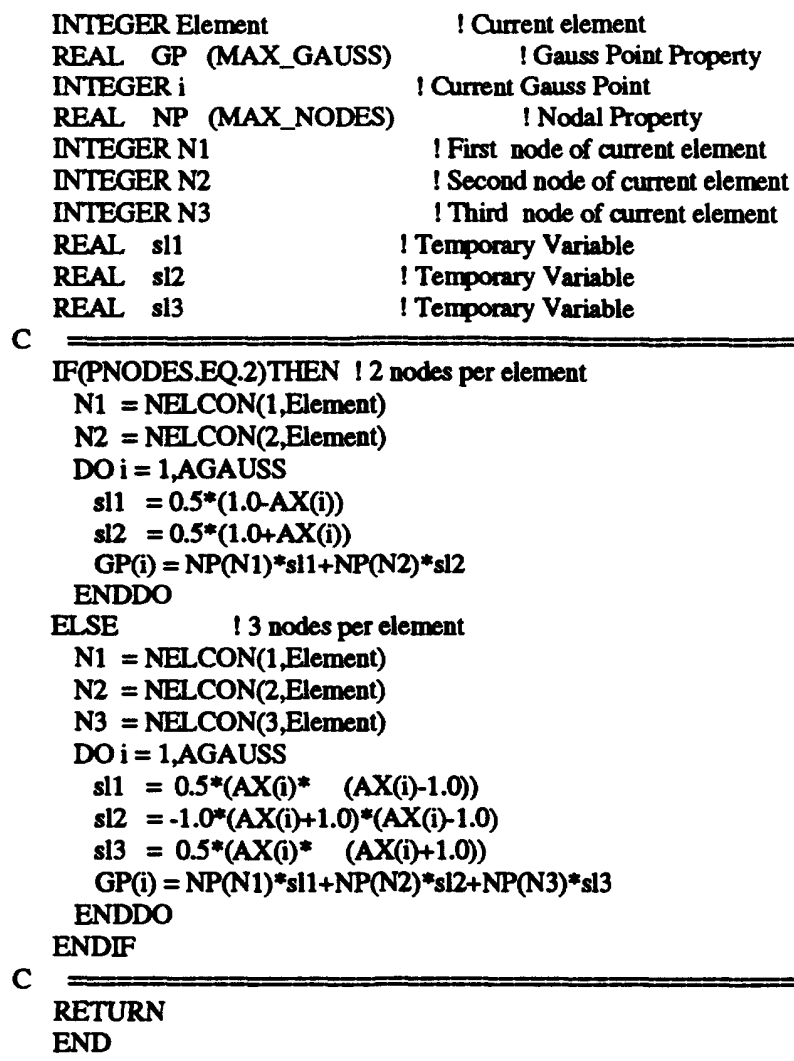

C IF(PNODES.EQ.2)THEN 12 nodes per element

\section{SUBROUTINE JSHAPE2(Element,NP,GP)}

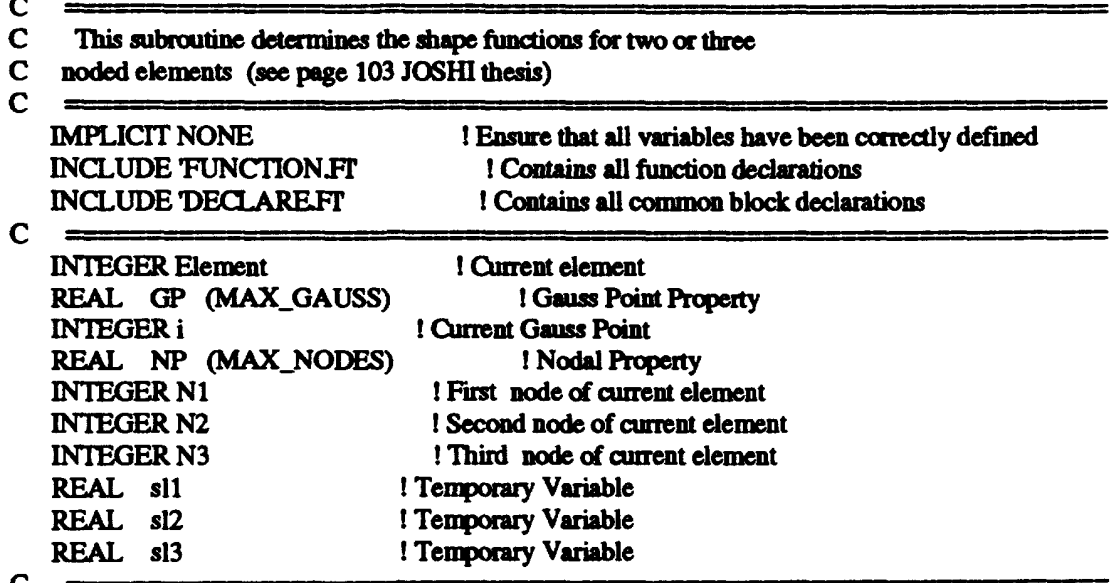

C

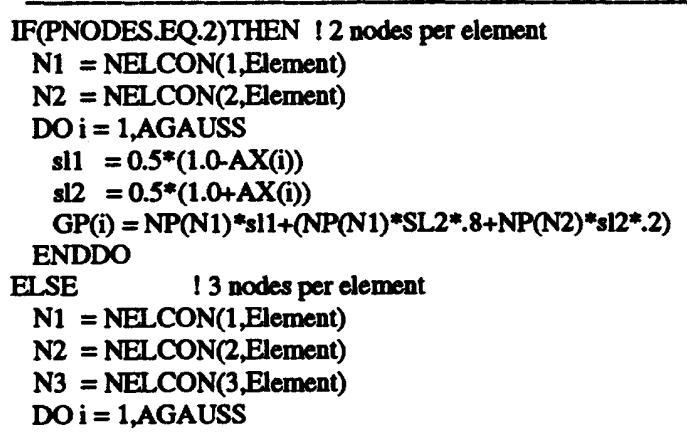




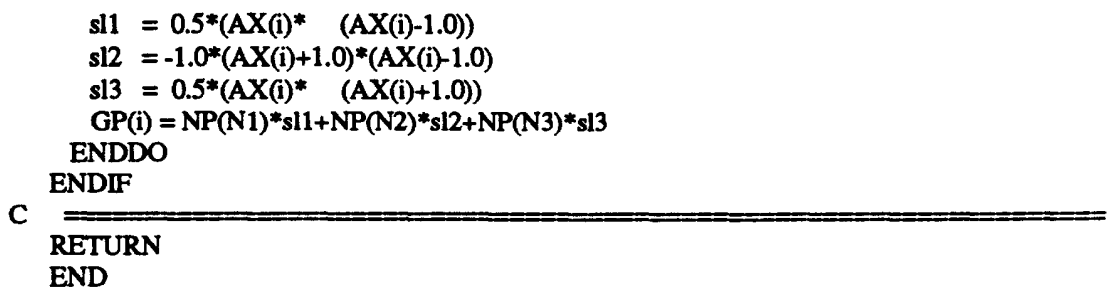

C This subroutine calculates the Leaf Area Index

$\mathrm{C}=$

\section{SUBROUTINE LeafAreaIndex}

C IMPLICIT NONE
INCLUDE FUNCTION.FI INCLUDE 'DECLARE.FT'
C ! defines nday in real value terms

C
C rday $=\mathrm{NDAY}^{*} 1.0 \quad$ IChange nday to a real number
IF(rday.LT.(EXP(XILAIDAY(1,1))-0.5))THEN
$\mathrm{LAI}=0.0 \quad$ ! if spec. but before grow. seas.
ELSEIF(rday.GT.(EXP(XILAIDAY(POINTS5(1),1))+0.5))THEN
$\mathrm{LAI}=0.0 \quad$ ! if spec. but after grow. season
ELSE
LAI = FN_POINT(1,POINTS5,XI_AIDAY,XI_AI,SPLINSL5,
1 rday) ! if spec. and in grow. season
ENDIF
IF(POINTS6(1).NE.0)THEN ! if mulch is specified
IF(rday.LT.(EXP(XMULCHDAY (1,1))-0.5))THEN
MULCH $=0.0 \quad !$ if spec. but before first spec
ELSEIF(rday.GT.(EXP(XMULCHDAY(POINTS6(1), 1))+0.5))THEN
MULCH $=0.0 \quad$ ! if spec. but after first spec
ELSE
MULCH = FN_POINT(1,POINTS6,XMULCHDAY,XMULCH,SPLINSL6,
1 rday) 1 if spec. and in grow. season
ENDIF
EISE I if veget. spec. but mulch not spec.
MULCH $=0.0$
ENDIF
else
$\mathrm{LAI}=0.0 \quad$ ! If veget. not specified
MULCH $=0.0$
endif
$\mathrm{C}=$
RETURN
END

\section{SUBROUTINE mprove(au,n,m1,m2,np,mp,al,mpl,indx,x,a,b)}


C to an improved set of values.

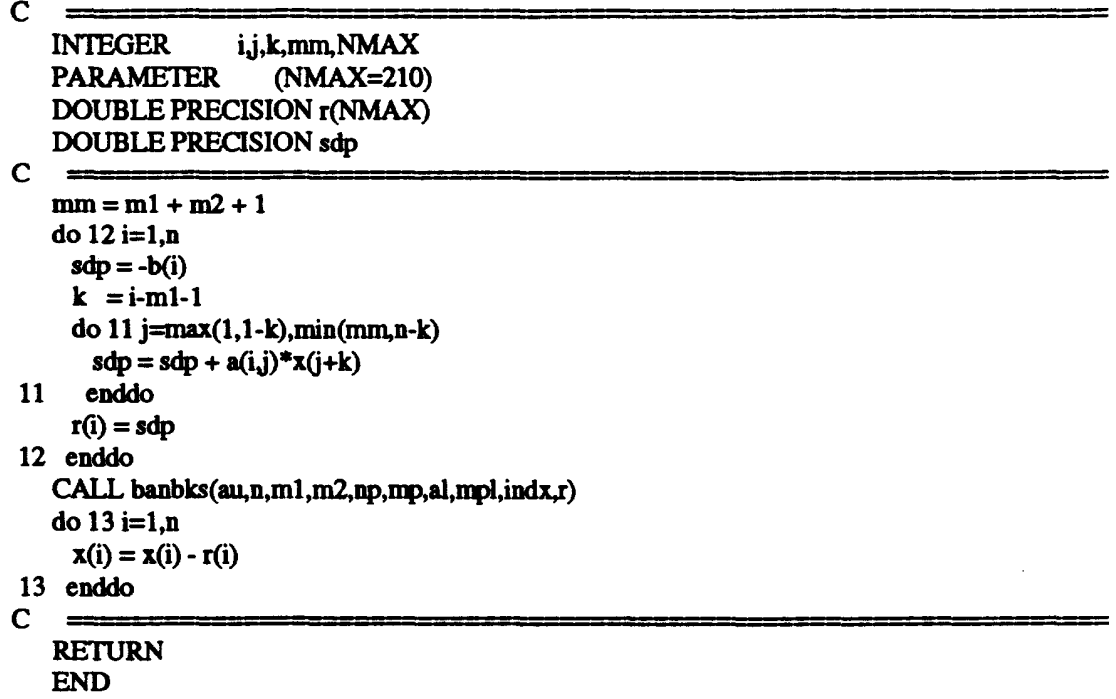

\section{SUBROUTINE PREPROCESSOR}

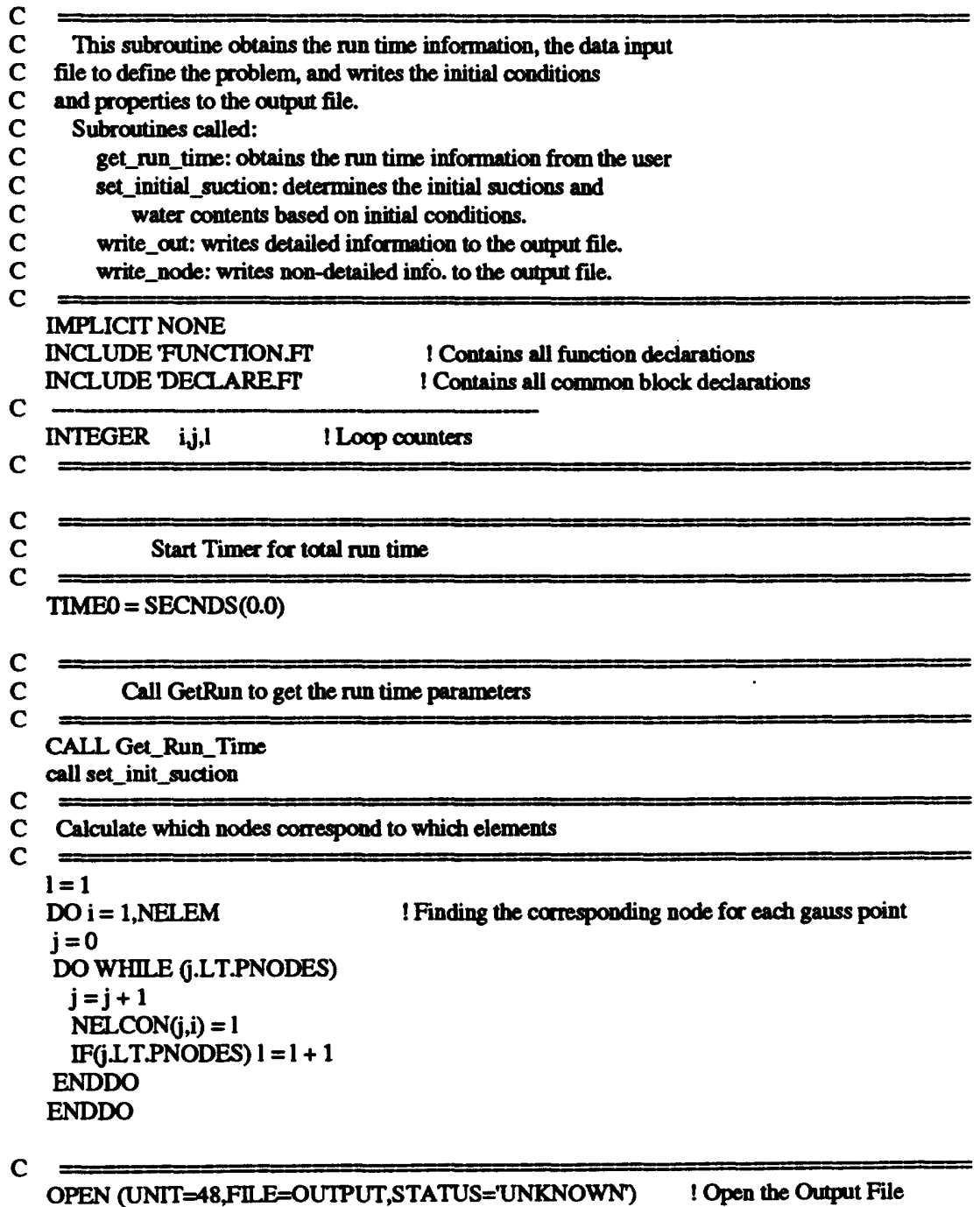



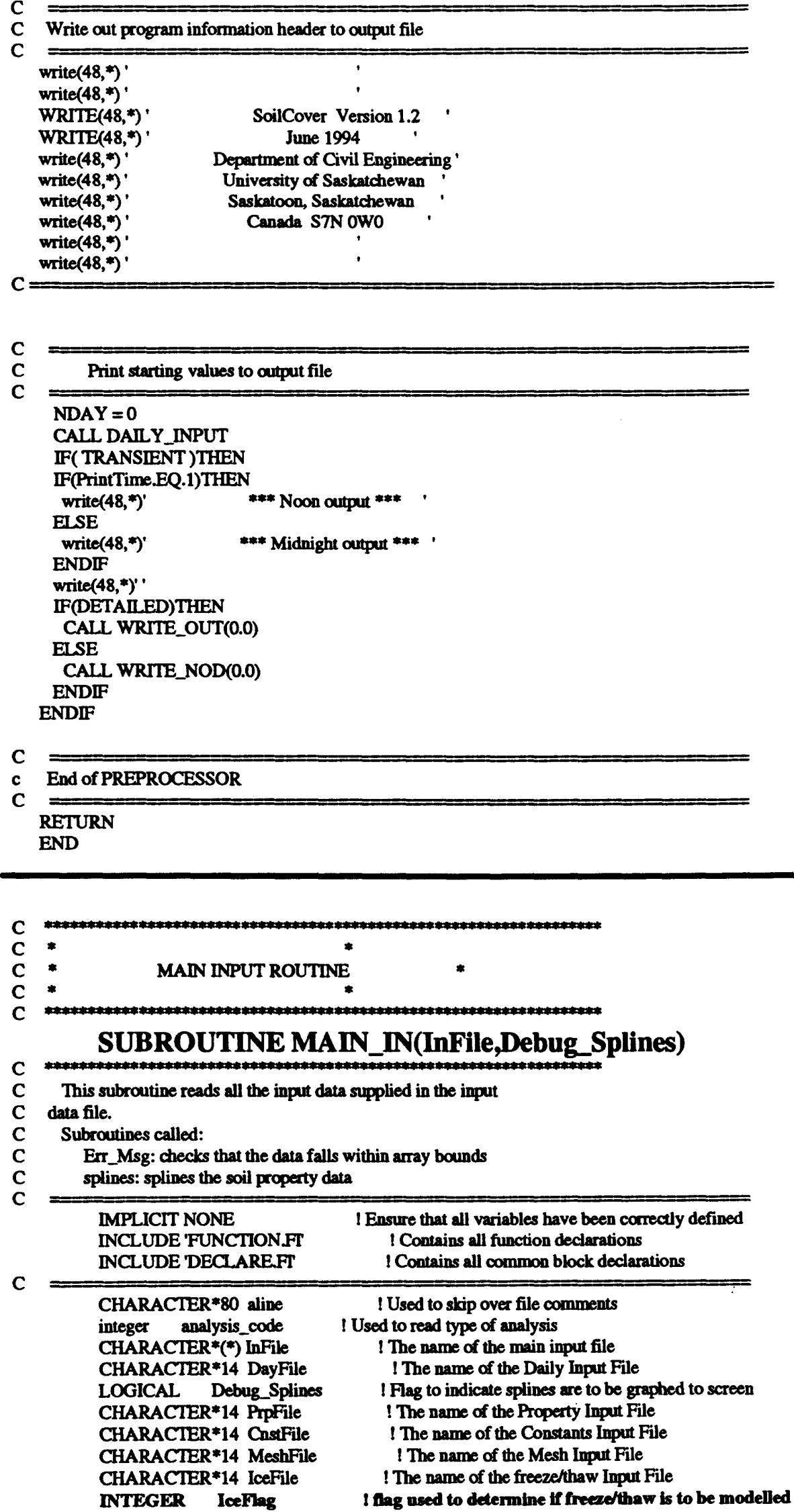


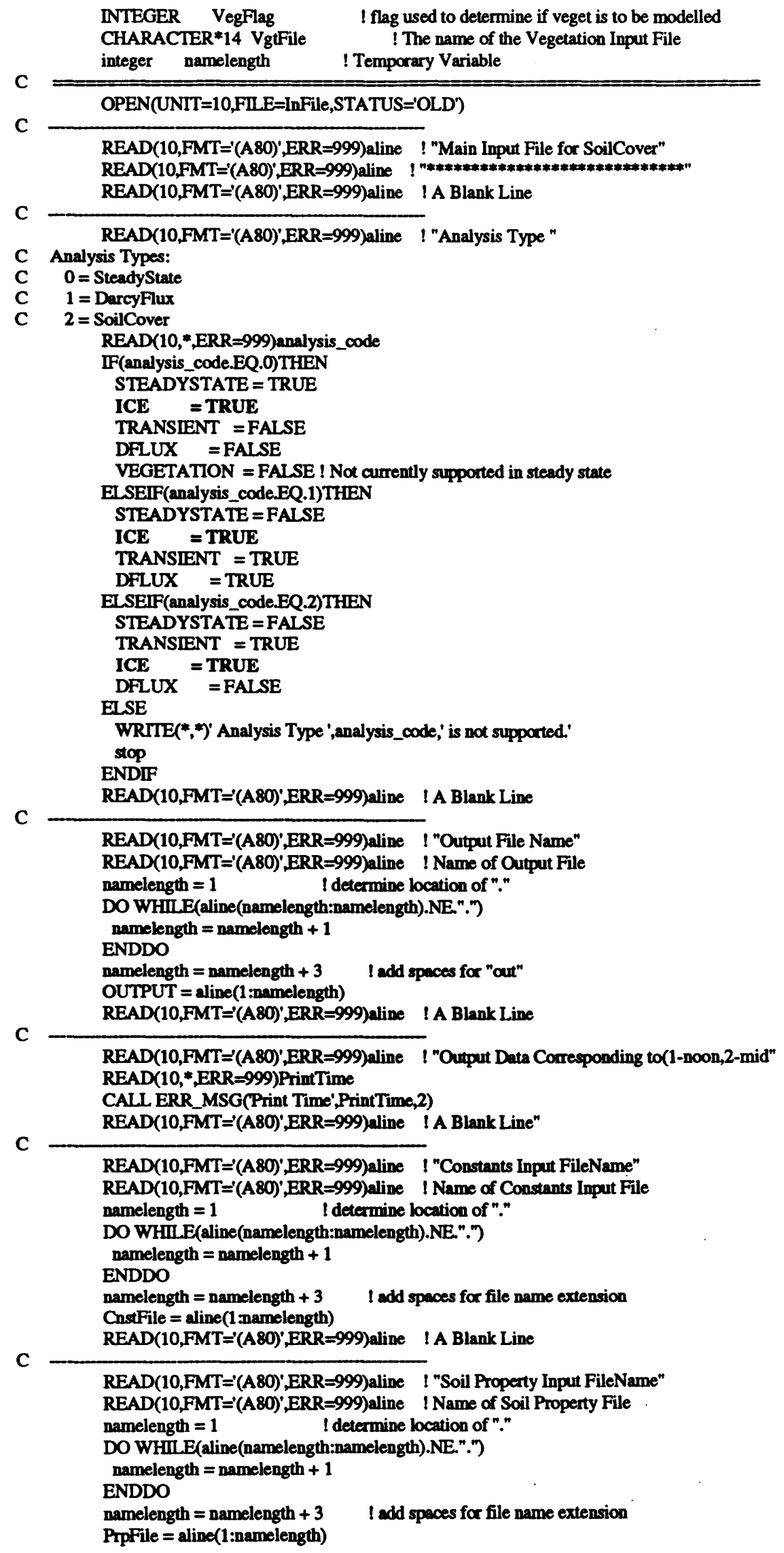




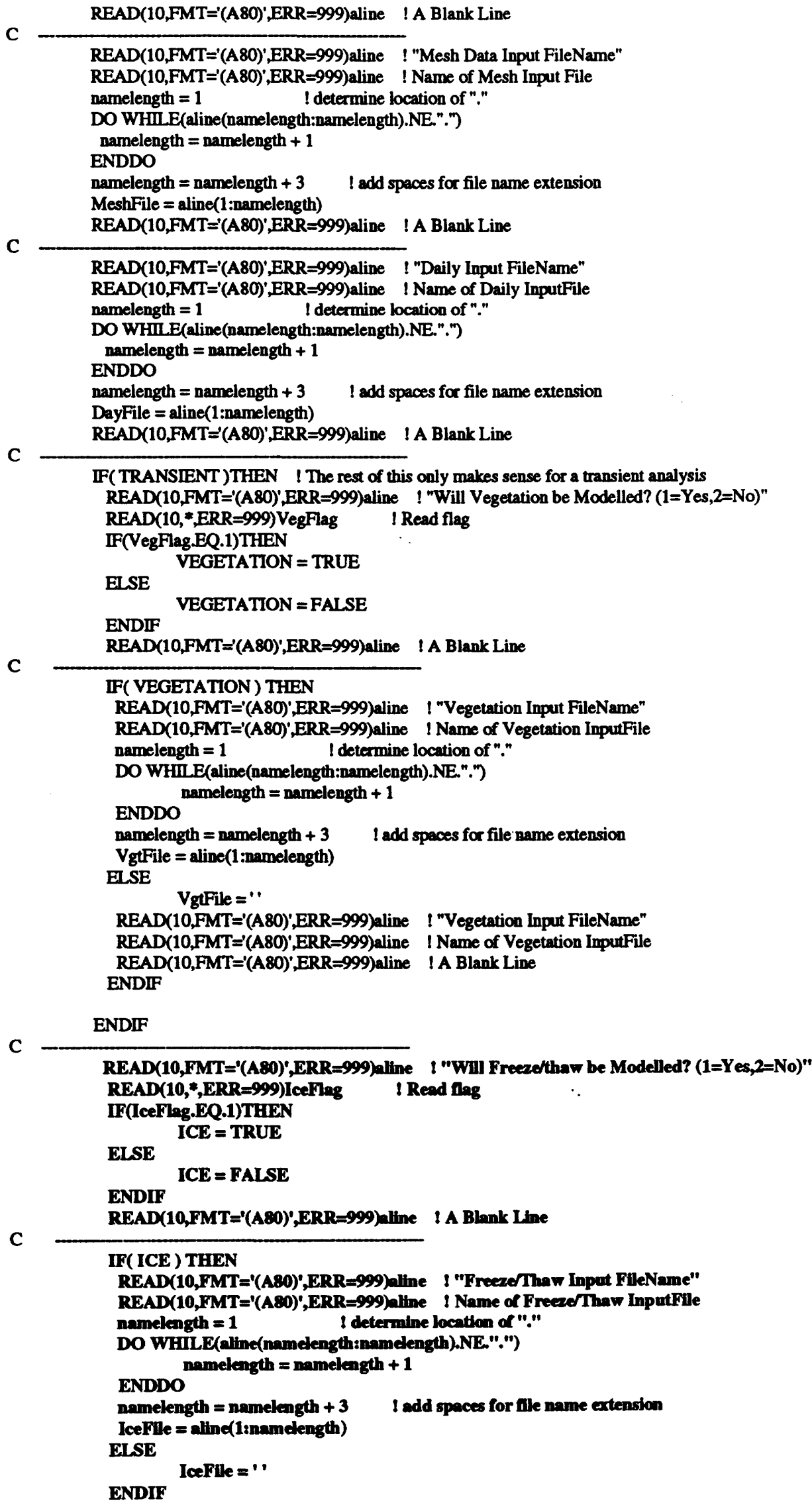




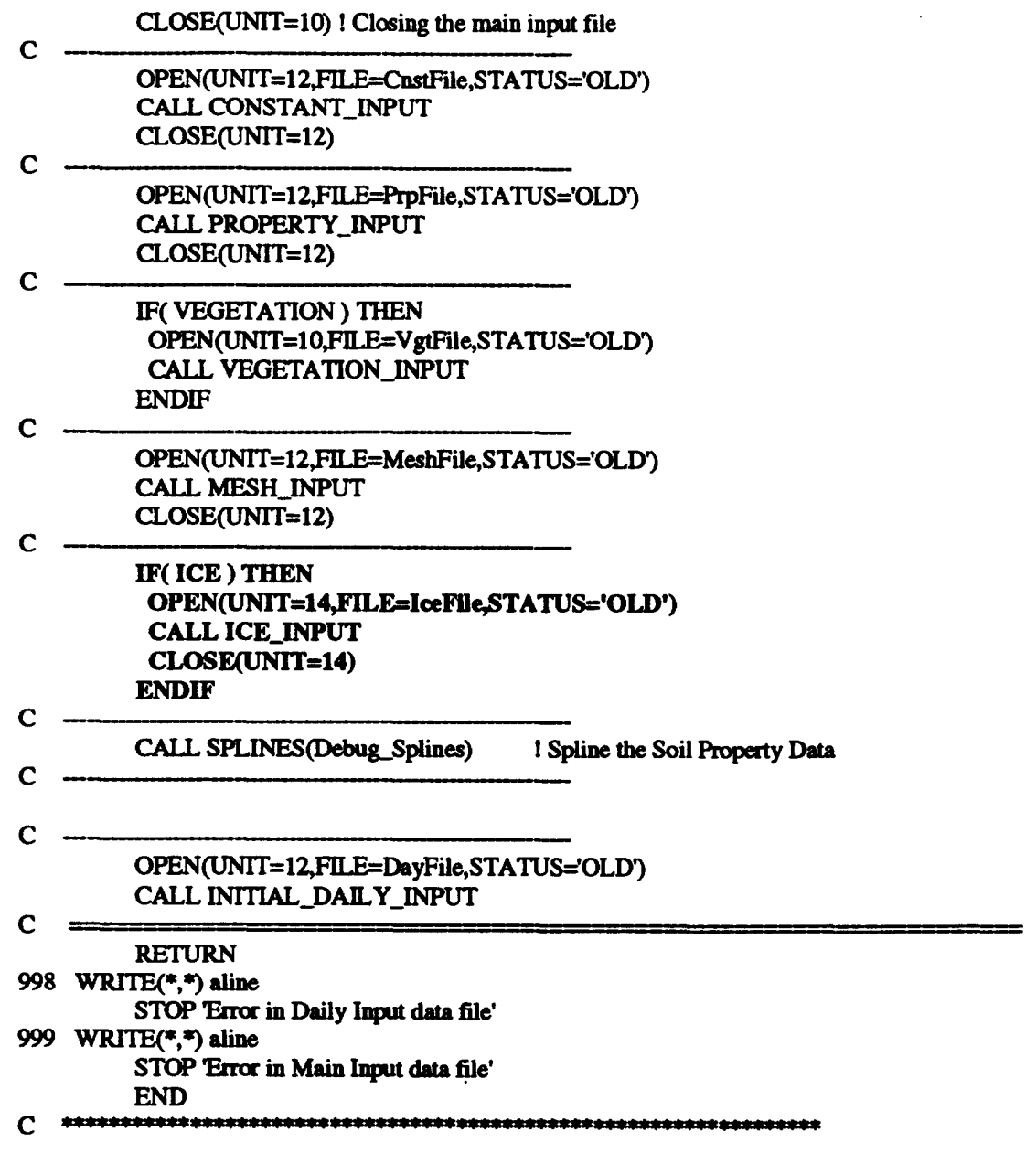

C

C $*$

C * SOIL PROPERTY INPUT ROUTINE *

C *

C

$\mathrm{C}=$ SUBROUTINE PROPERTY_INPUT

C This subroutine reads all the soil propenty input data supplied

C in the soil property input data file.

C Subroutines called:

C Err_Msg: checks that the data falls within array bounds

C E IMPLICIT NONE I Ensure that all variables have been correctly defined INCLUDE FUNCTION.FT I Contains all function declarations INCLUDE 'DECLARE.FT ! Contains all common block declarations

C CHARACTER $* 80$ aline ! Used to skip over file comments INTEGER j ILoop Counter integer type the soil type number integer wctype I specifies whether user inputs soil properties in Grav. or Vol. w/c real xwc Itemporary water content variable

C READ(12,FMT='(A80)',ERR=999)aline ! "SoilProperty InputFile for SoilCover"

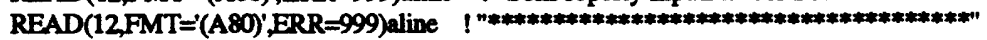




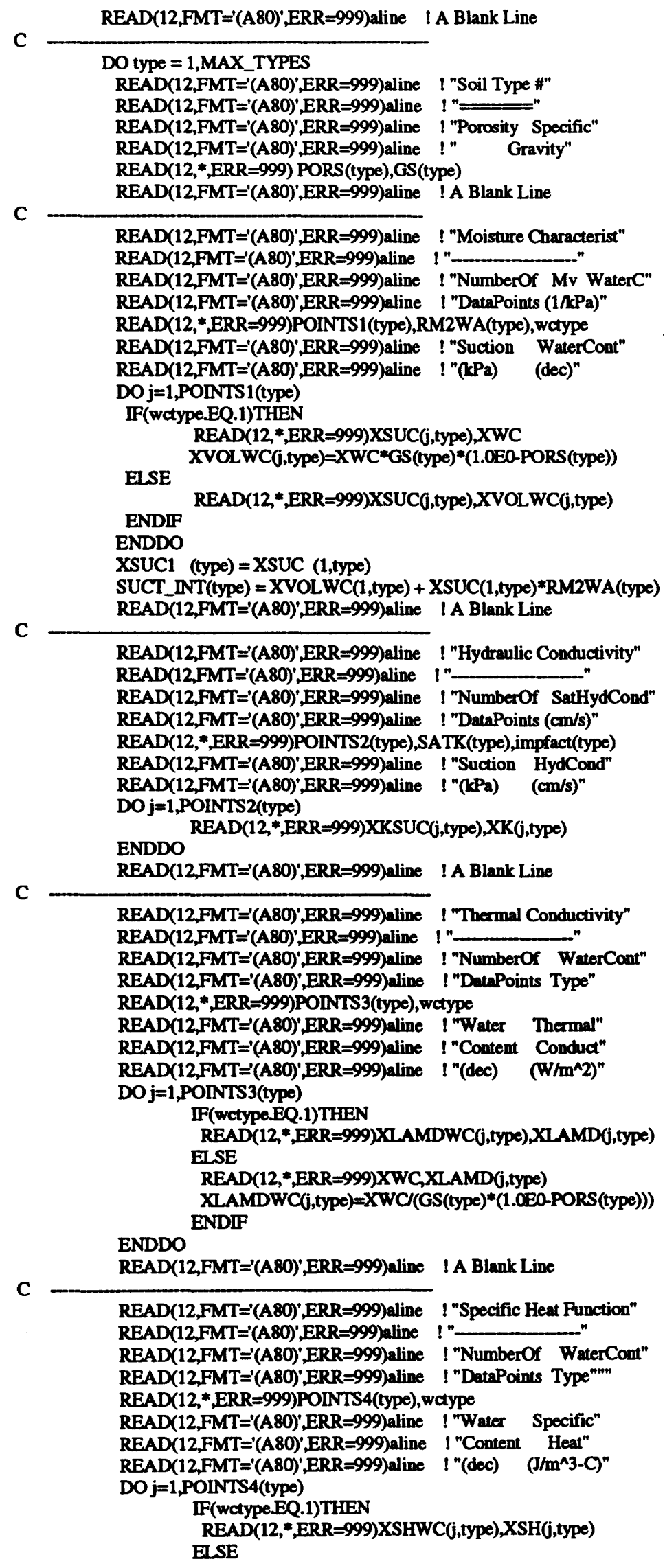



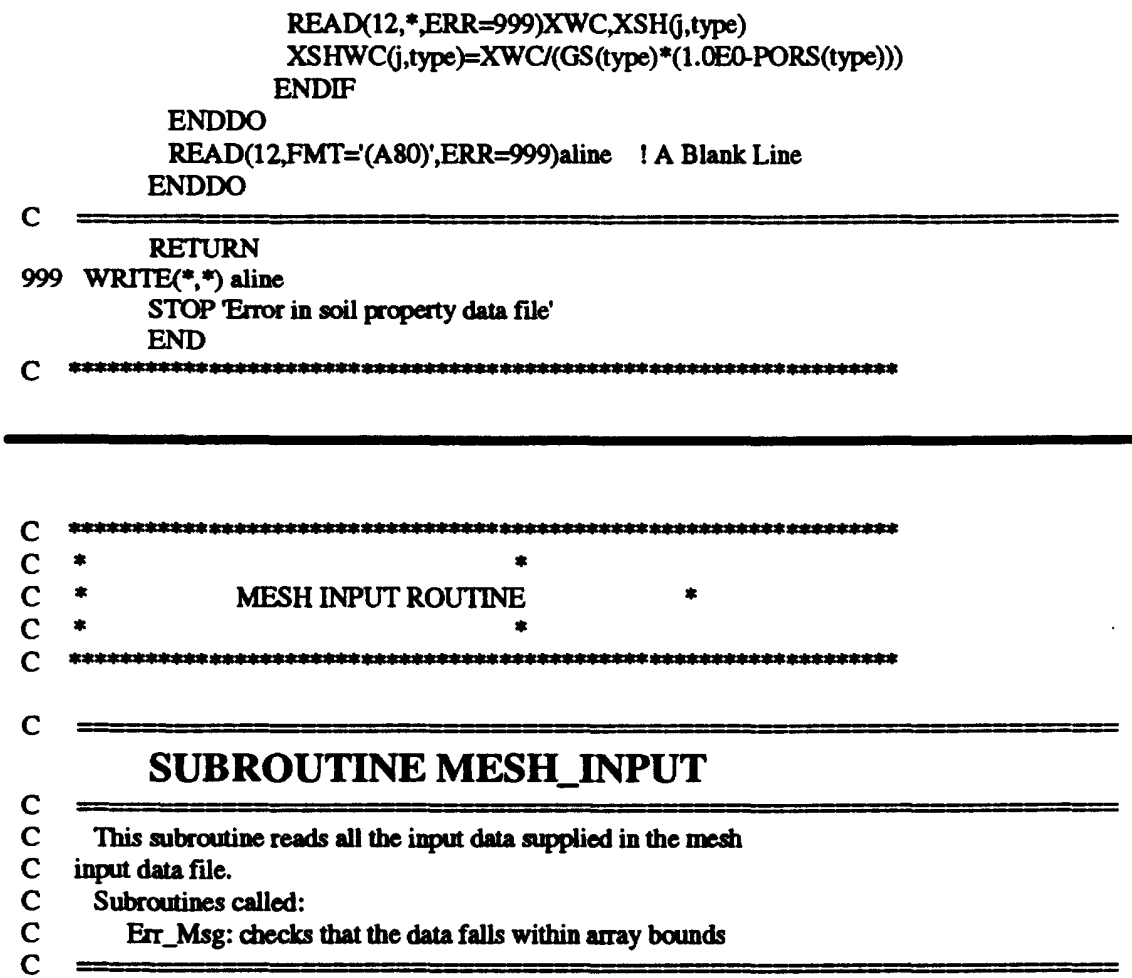


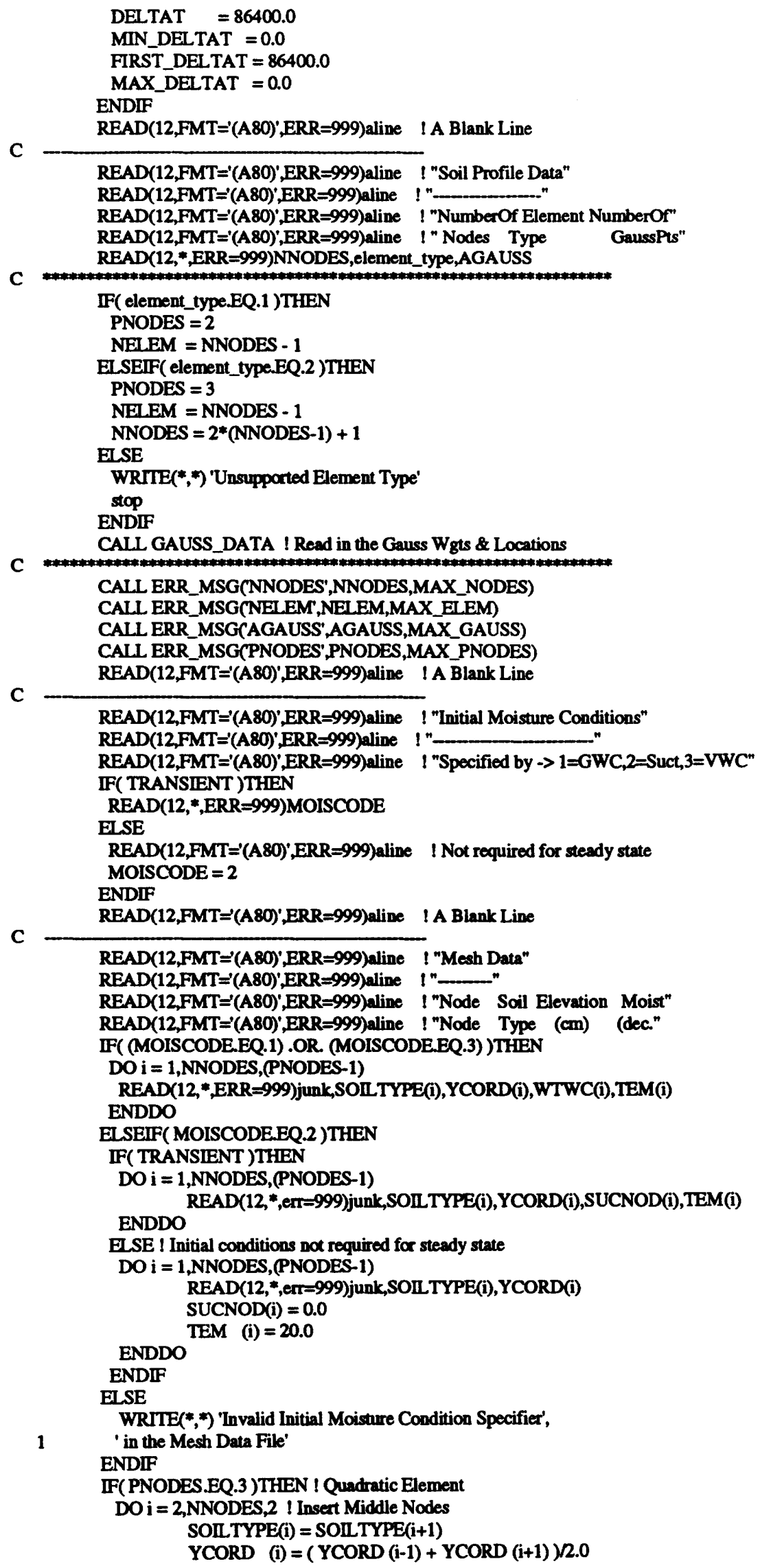


ENDDO

READ(10,FMT='(A80)',ERR=999)aline ! A Blank Line

READ(10,FMT='(A80)',ERR=999)aline ! "Daily Root Depth Data"

READ(10,FMT='(A80)',ERR=999)aline ! "

READ $(10, F M T=(A 80)$ ',ERR=999)aline ! "Day TopNode BottomNode"

\section{C $=$ RETURN}

999 WRITE(*,*) aline

STOP 'Erroc in Vegetation data file'

END

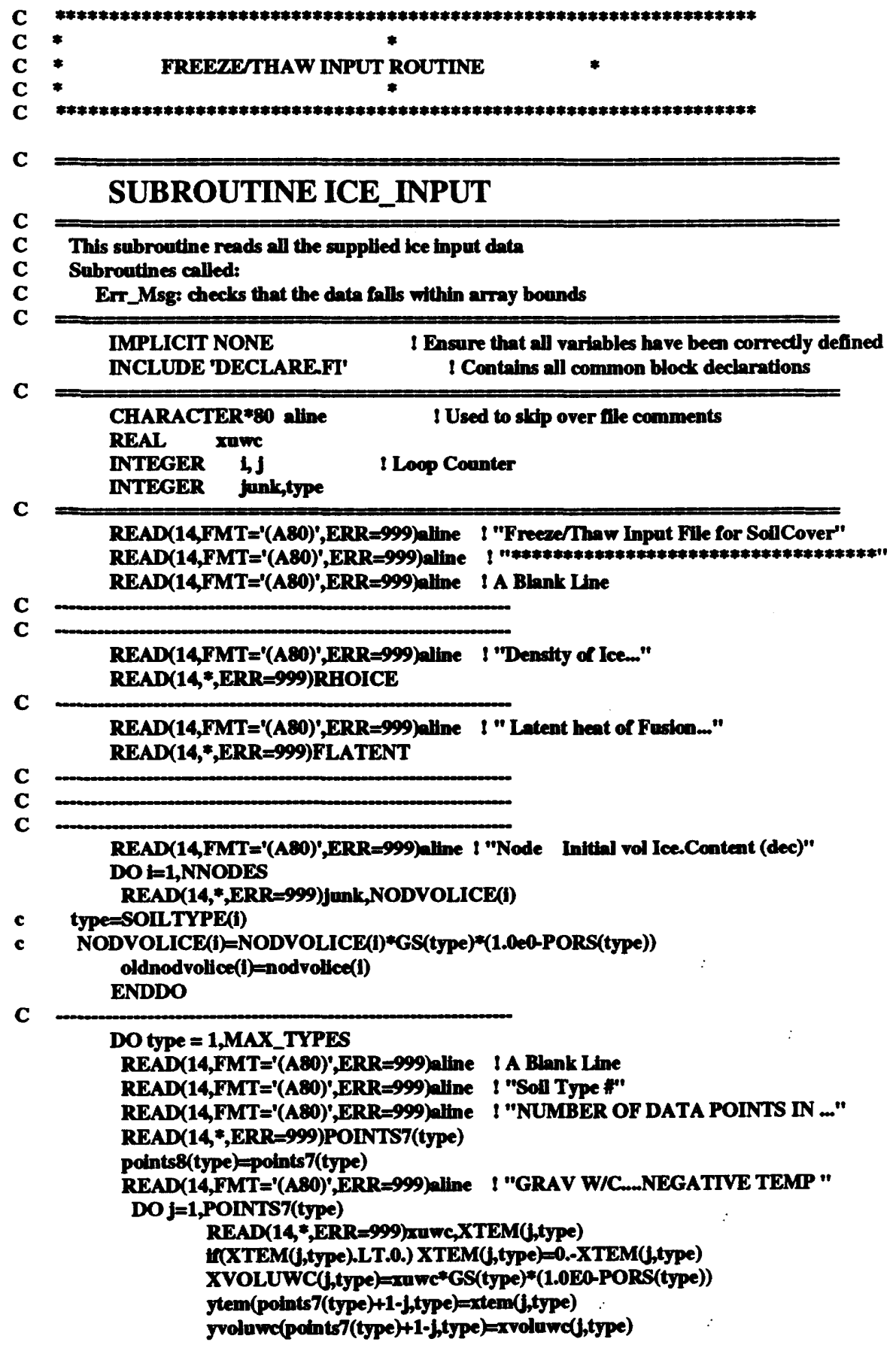


ENDDO

READ(14,FMT='(A80)',ERR=999) )aline I A Blank Line

READ(14,FMT='(A80)',ERR=999)aline I "NUMBER OF DATA POINTS IN ..."

READ(14*,ERR=999)POINTS9(type)

READ(14,FMT='(A80)',ERR=999)aline $f$ "vol W/C..ndsuc / dtem "

DO j=1,POINTSY (type)

ENDDO

READ(14,*,ERR=999)GVOLUWC(j,type),XGG(j,type)

ENDDO

RETURN

$999 \operatorname{WRTTE}(*, *)$ aline

STOP 'Error in freeze/thaw data fle'

END

C

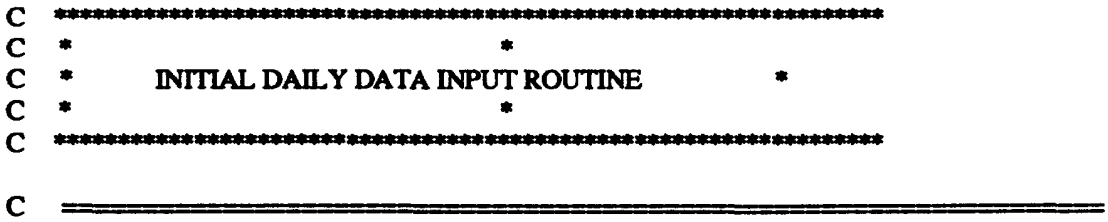

\section{SUBROUTINE INITIAL_DAILY_INPUT}

C This subroutine reads the climate and boundary condition data

C on a daily basis.

C Subroutines called:

C Err_Msg: checks that the data falls within array bounds

C

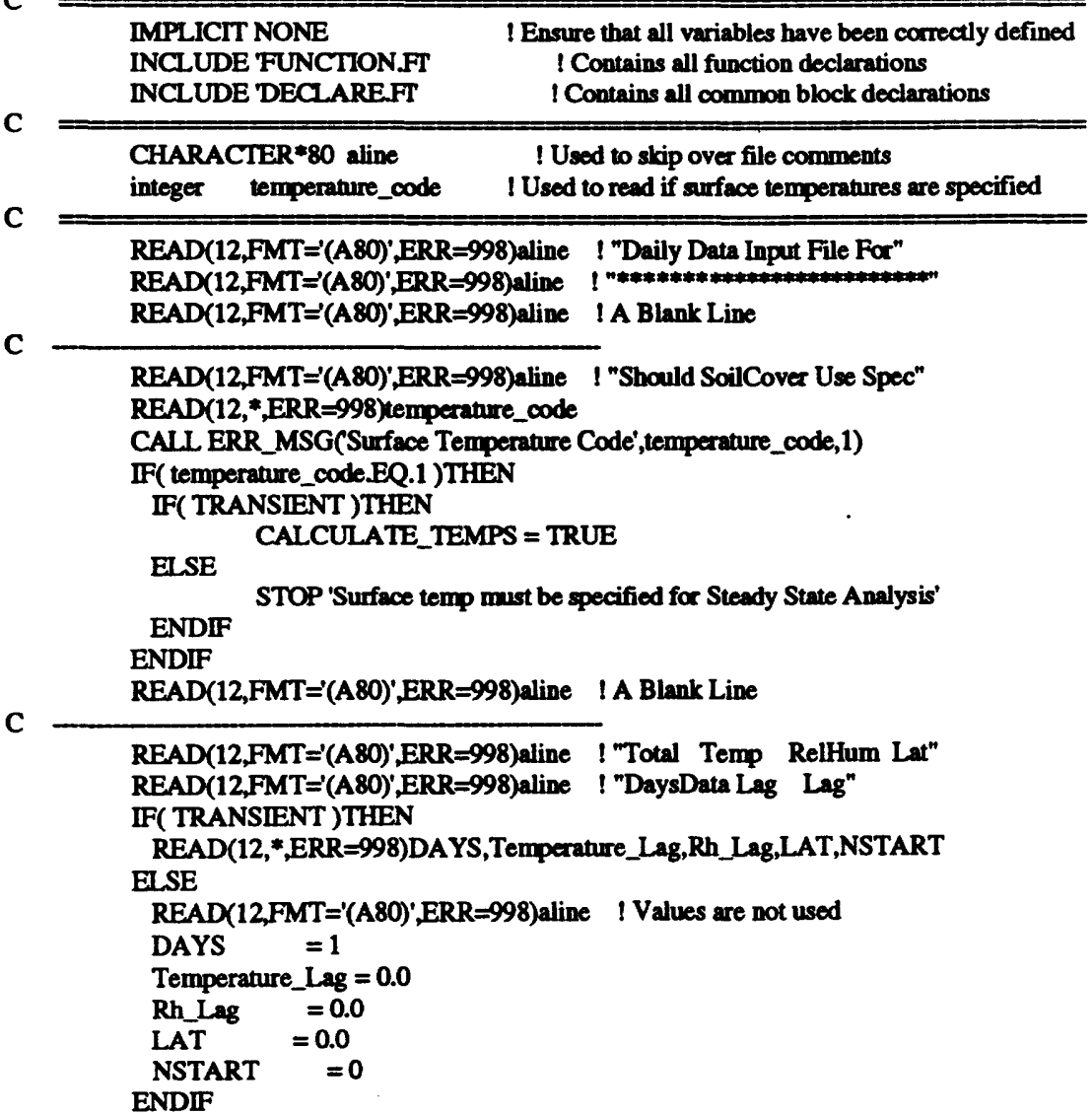


CALL ERR_MSG(DAYS',DAYS,256000)

CALL ERR_MSG(Days Past Jan.',NSTART,365)

READ(12,FMT='(A80)',ERR=998)aline ! A Blank Line

READ(12,FMT='(A80)',ERR=998)aline ! "Daily Data"

READ(12,FMT='(A80)',ERR=998)aline !"

READ(12,FMT='(A80)',ERR=998)aline ! Headings Row \#1 Line

READ(12,FMT='(A80)',ERR=998)aline ! Headings Row \#2 Line

READ(12,FMT='(A80)',ERR=998)aline I Headings Unit Line

RETURN

998 STOP 'Error in initial part of daily input data file'

END

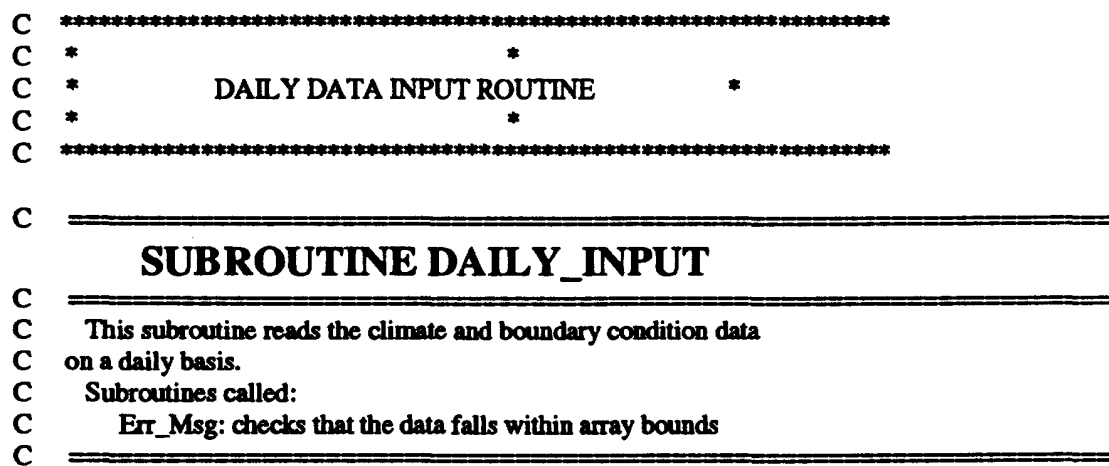
IMPLICIT NONE I Ensure that all variables have been correctly defined INCLUDE 'FUNCTION.FT' IContains all function declarations INCLUDE DECLAREFT IContains all common block declarations
C

\begin{tabular}{ll}
\hline $\begin{array}{l}\text { integer bot_type } \\
\text { real bot_value }\end{array}$ & ! specifies whether top node has vol. wc head input \\
INTEGER i & ! Loop Counter \\
INTEGER j & ! Loop Counter \\
INTEGER junk & I Used to skip over integer in input file \\
integer top_type & ! specifies whether bottom head boundary inpert as vol. wc \\
real top_value & ! the actual boundary condition \\
\hline
\end{tabular}
DO $\mathrm{i}=1,2$
NODEB $(1, i)=1$
NODEB $(2, \mathrm{i})=$ NNODES
NODEN $(1, \mathrm{i})=1$
NODEN $(2, i)=$ NNODES
TEMPAMAX $(i)=$ TEMPAMAX $(i+1)$
TEMPAMIN (i) = TEMPAMIN $(i+1)$
SOLAR (i) $=$ SOLAR $(i+1)$
RH_MAX (i) $=$ RH_MAX $(i+1)$
RH_MIN (i) $=$ RH_MIN $(i+1)$
WIND $\quad(i)=$ WIND $\quad(i+1)$
DURATION $(i)=$ DURATION $(i+1)$
RootTop (i) $=$ RootTop $(i+1)$
RootDepth(i) = RootDepth $(i+1)$
EBW $(1, i)=$ EBW $(1, i+1)$
EBW $(2, i)=E B W \quad(2, i+1)$
EBH $(1, i)=$ EBH $\quad(1, i+1)$
EBH $\quad(2, i)=E B H \quad(2, i+1)$
QW $\quad(1, \mathrm{i})=$ QW $\quad(1, \mathrm{i}+1)$
QW $\quad(2, \mathrm{i})=\mathrm{QW} \quad(2, \mathrm{i}+1)$
VFLUXPAN $(i)=$ VFLUXPAN $(i+1)$
ENDDO
C
IF( NDA Y.LT.DAYS )THEN ! If there is more data to read
READ(12,*,ERR=999)junk, TEMPAMAX(3),TEMPAMIN(3),SOLAR(3),

1 RH_MAX(3),RH_MIN(3),WIND(3),top_type,top_value,DURATION(3),

1 bot_type,bot_value,EBH(1,3),EBH(2,3), nexttoptemp ! nexttoptemp is next days user defined surface temp. IF(CALCULATE_TEMPS)THEN 


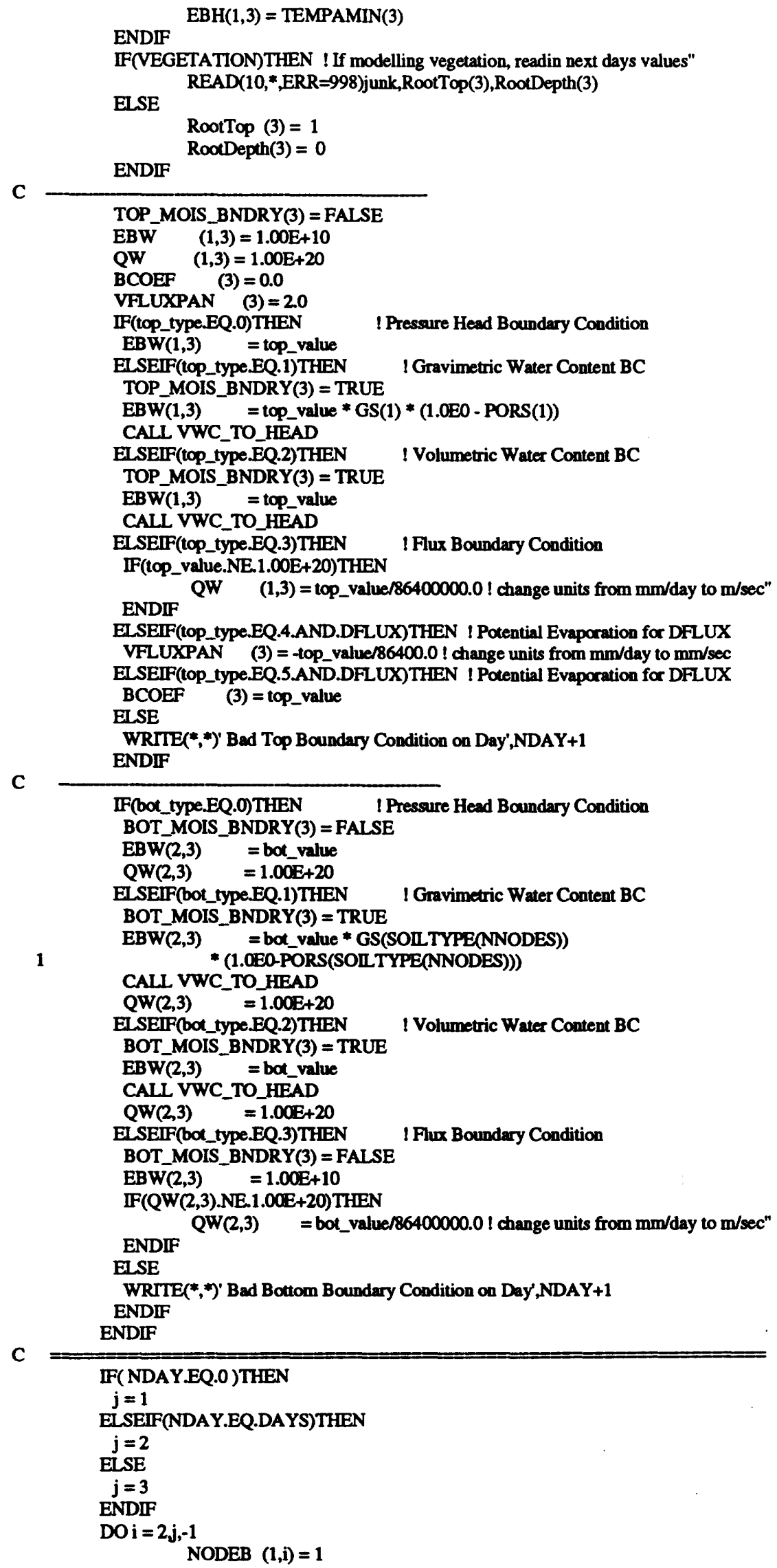


NODEB $(2, \mathbf{i})=$ NNODES

NODEN $(1, \mathrm{i})=1$

NODEN $(2, i)=$ NNODES

TEMPAMAX (i) = TEMPAMAX (3)

TEMPAMIN (i) = TEMPAMIN (3)

SOLAR (i) = SOLAR (3)

RH_MAX (i) $=$ RH_MAX (3)

RH_MIN (i) $=$ RH_MIN (3)

WIND (i) = WIND (3)

DURATION (i) = DURATION (3)

RootTop (i) $=$ RootTop (3)

RootDepth $(i)=$ RootDepth $(3)$

EBW $(1, \mathrm{i})=\mathrm{EBW} \quad(1,3)$

EBW $(2, \mathrm{i})=$ EBW $(2,3)$

EBH $(1, \mathrm{i})=\mathrm{EBH} \quad(1,3)$

EBH $(2, i)=$ EBH $(2,3)$

QW $\quad(1, \mathrm{i})=\mathrm{QW} \quad(1,3)$

QW $\quad(2, \mathbf{i})=\mathrm{QW} \quad(2,3)$

ENDDO

VFLUXPAN (i) = VFLUXPAN (3)

\section{$C=$ RETURN}

998 STOP 'Error in daily Root Depth Data File'

999 STOP 'Error in daily input data file'

END
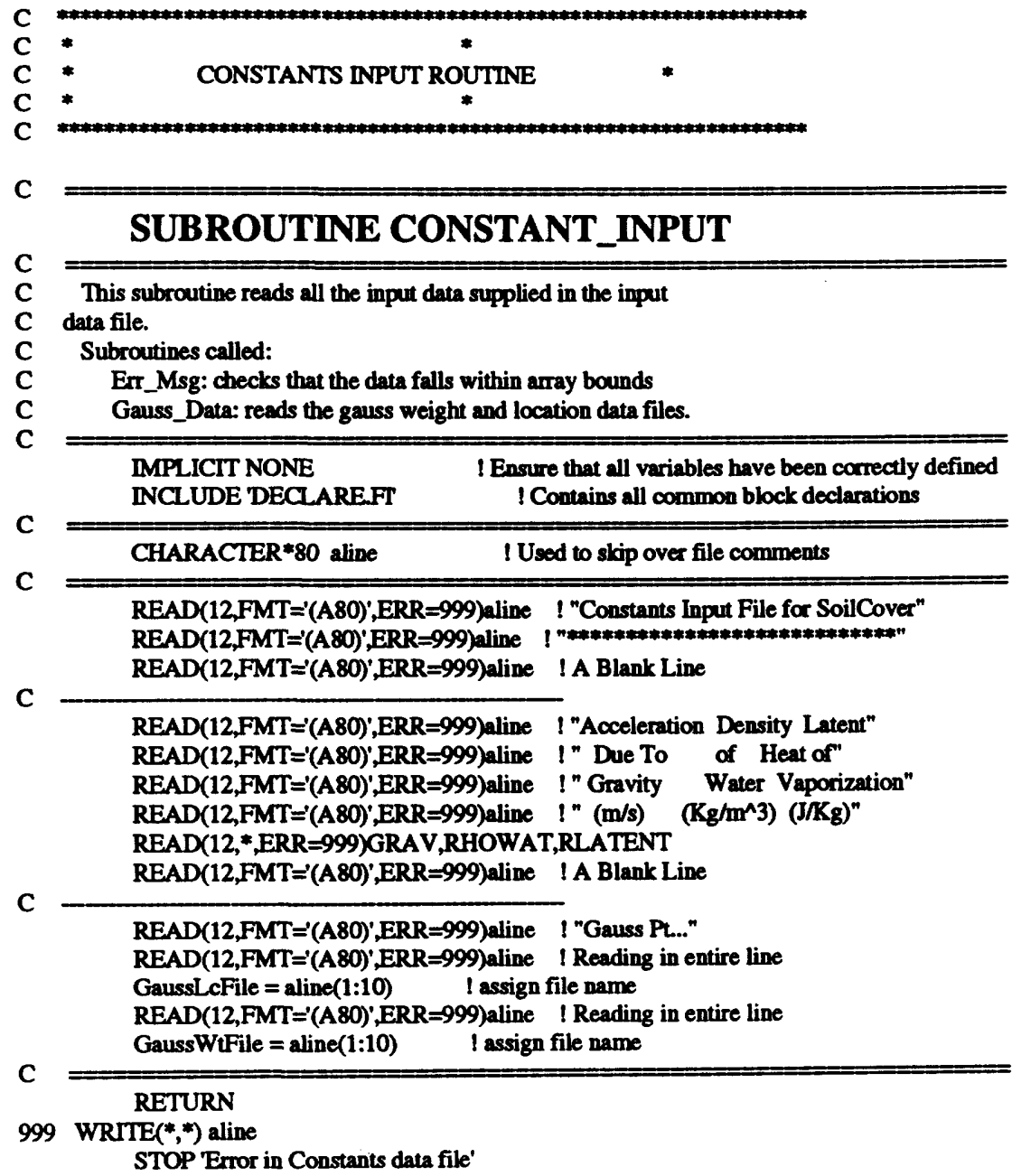
END

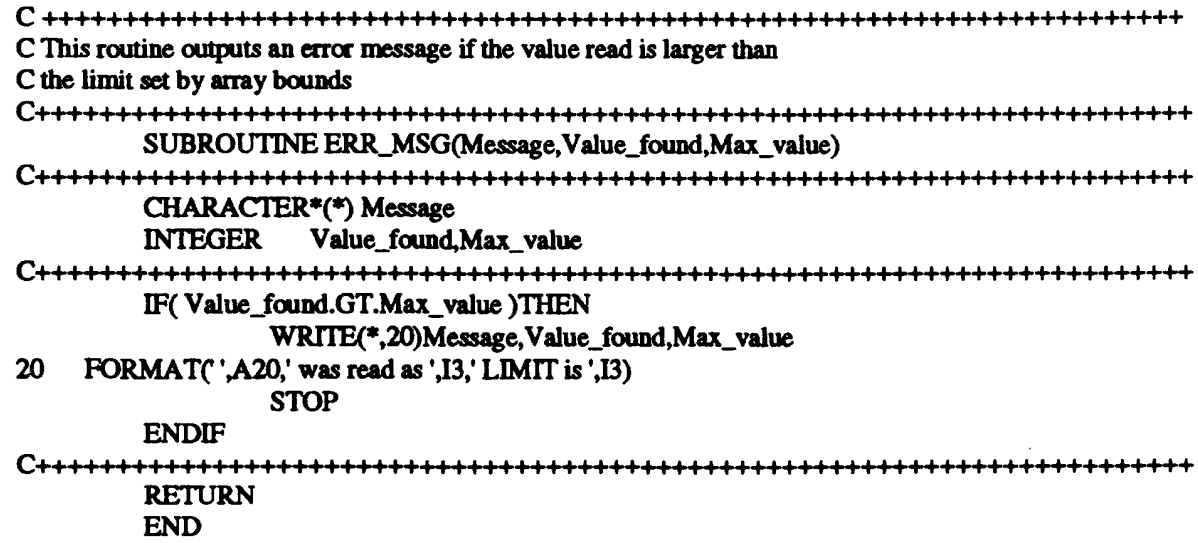

\section{SUBROUTINE RELAXATION}

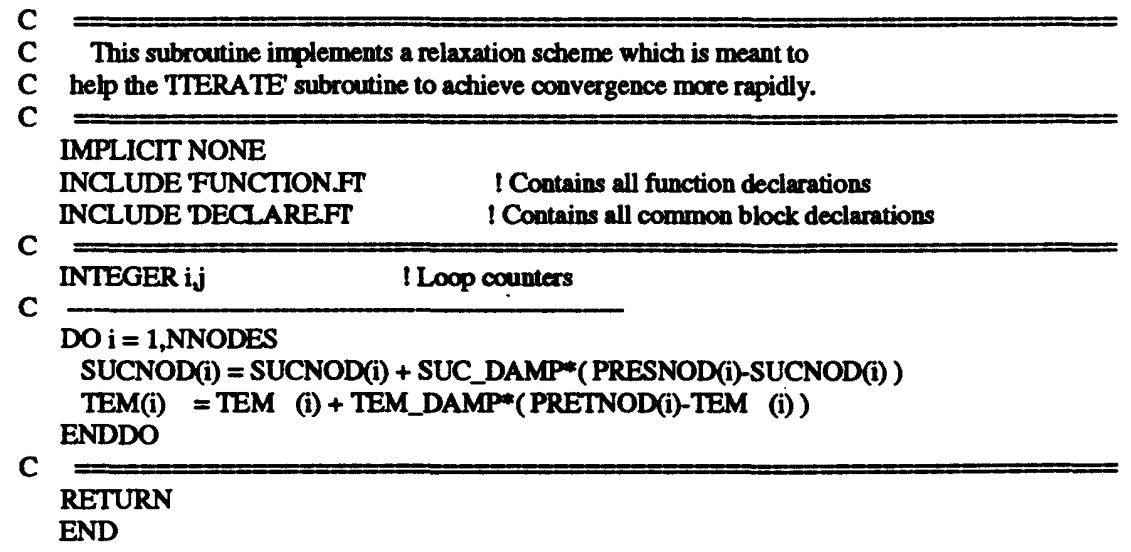

\section{SUBROUTINE REVERSE_SPLINES ! suction vs water content}

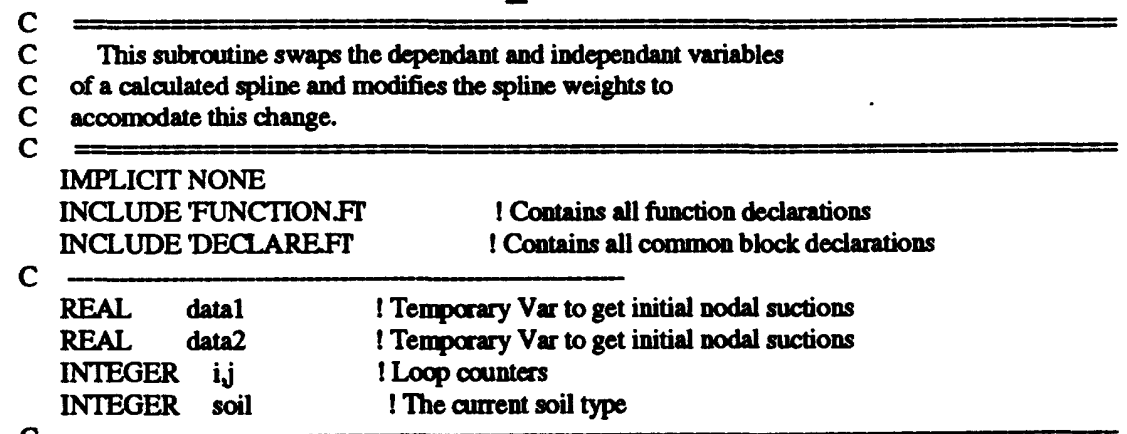

C do soil=1,MAX TYPES

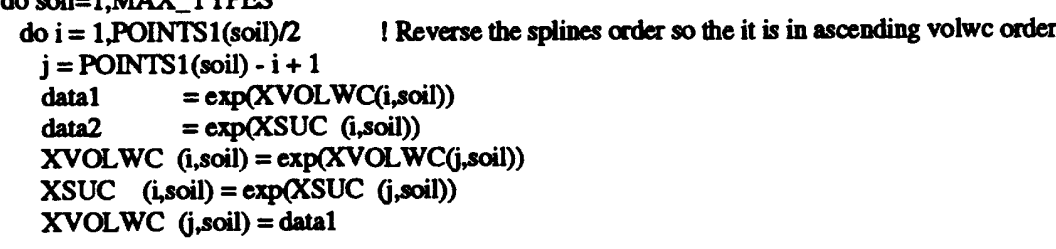




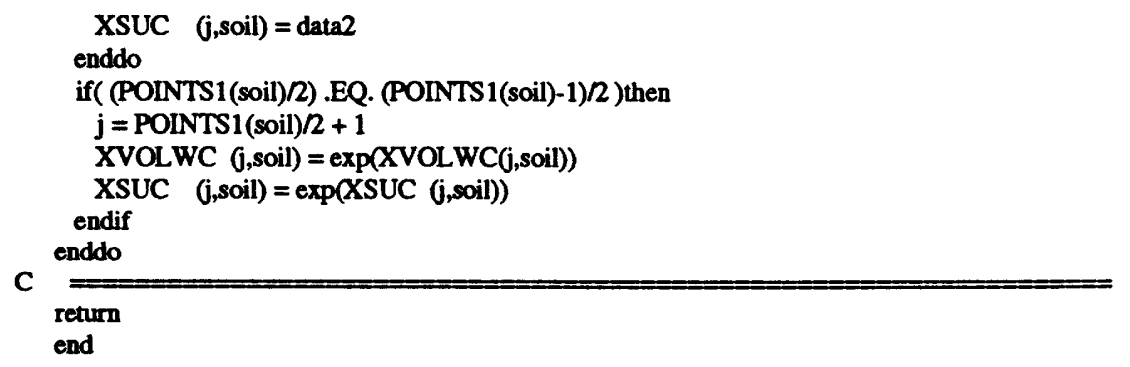

\section{SUBROUTINE REVERSE_SPLINES2 ？ temperature vs water content}

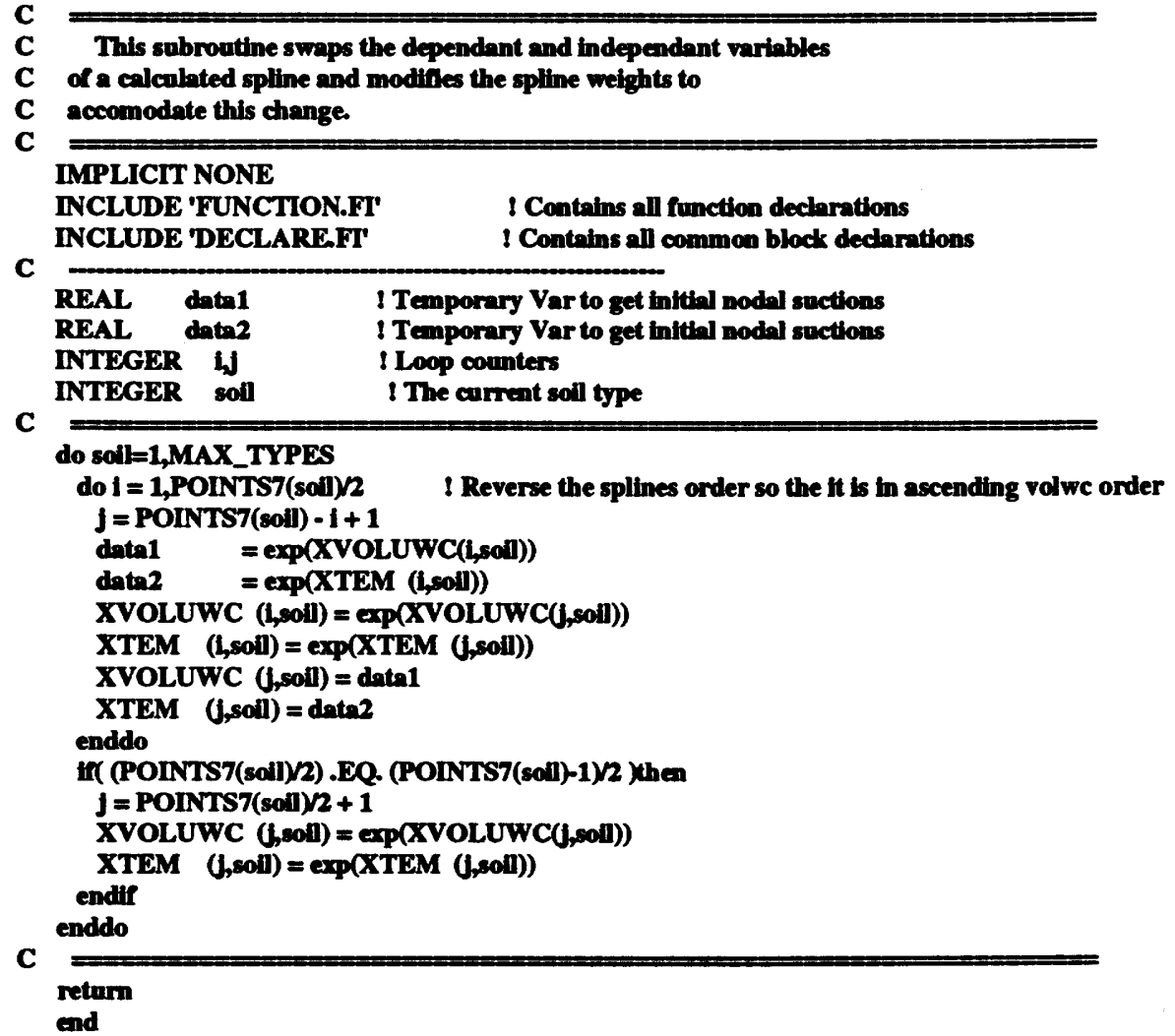

\section{SUBROUTINE SET_INITIAL_SETTINGS}

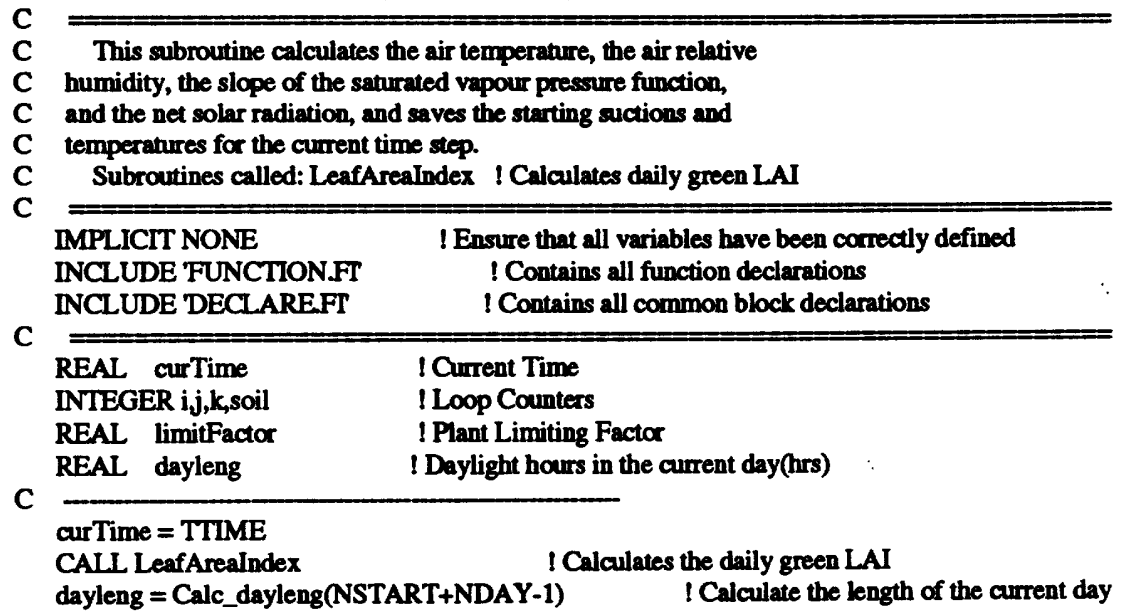


TEMPAIR $=$ Calc_AIRTemp(dayleng, curTime)

RHAIR1 = Calc_AirRH(dayleng,curTime)

! (K) AIR TEMP

! Relative Humidity of the air

SLPOT $1=0.1^{*}\left(0.00815^{*}(\text { TEMPAIR-273.0 })+0.8912\right)^{* * 7}$ ! this is a emperical method to calculate the slope of the satvp - temp curve (Kpa/cel)

PVAIR1 = Calc_SatVP(TEMPAIR)*RHAIR1/10.0 ! Vapour Pressure of the air

QSTAR = Calc_NETRAD(dayleng)

I Net solar radiation in $\mathrm{mm} /$ day

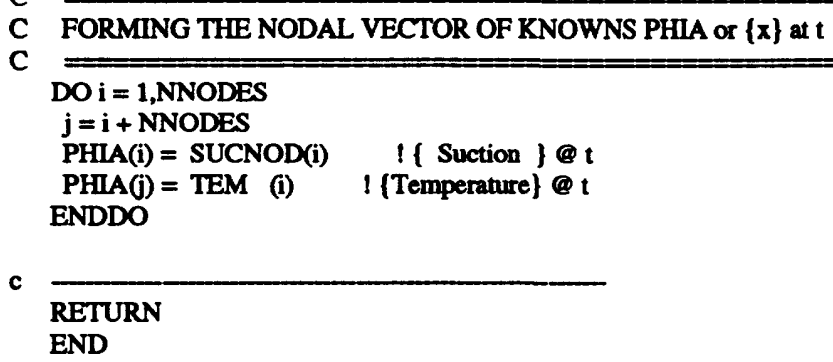

\section{SUBROUTINE SET_INIT_SUCTION}

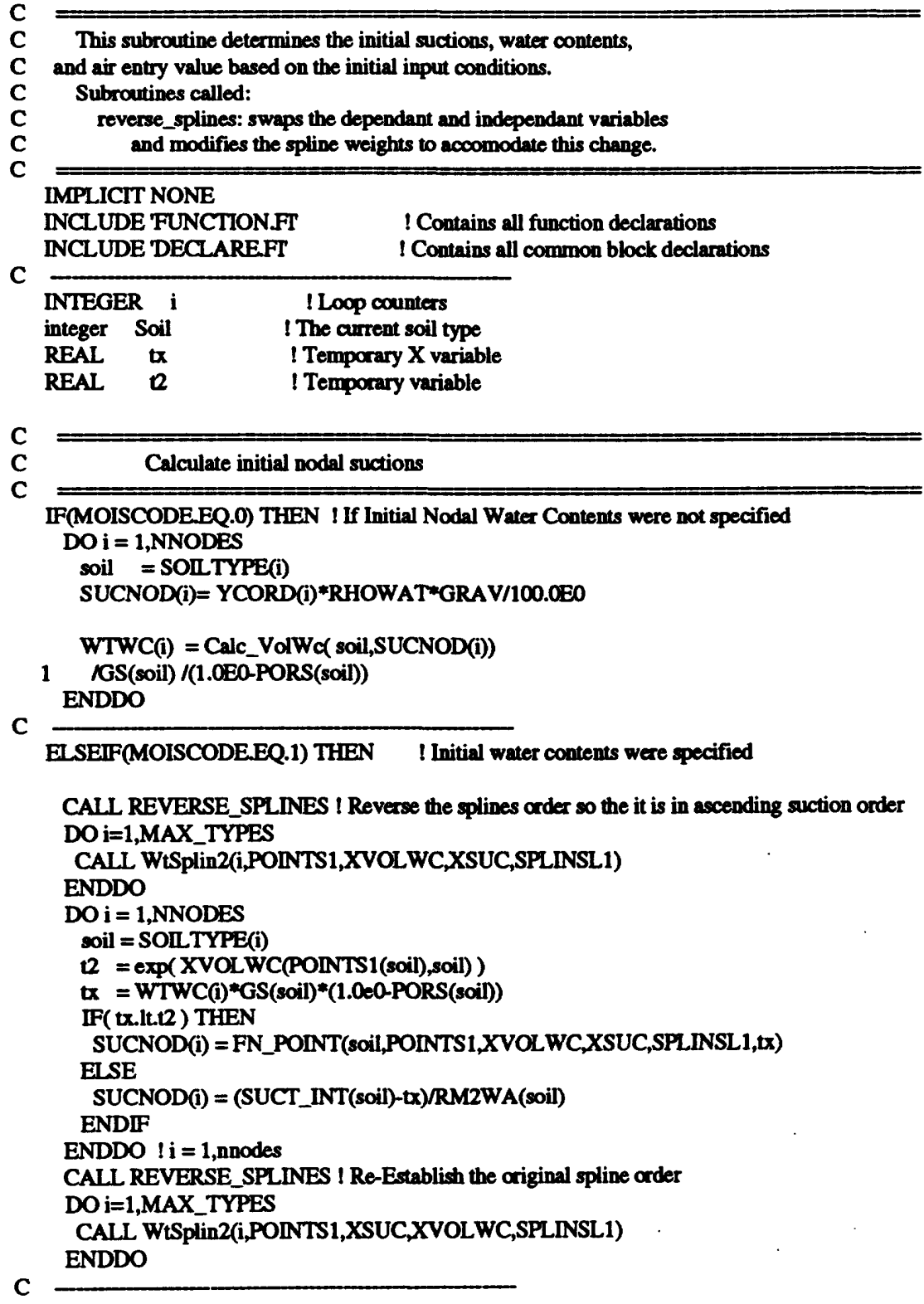


ELSEIF(MOISCODE.EQ.2) THEN ! Initial Pressures were specified

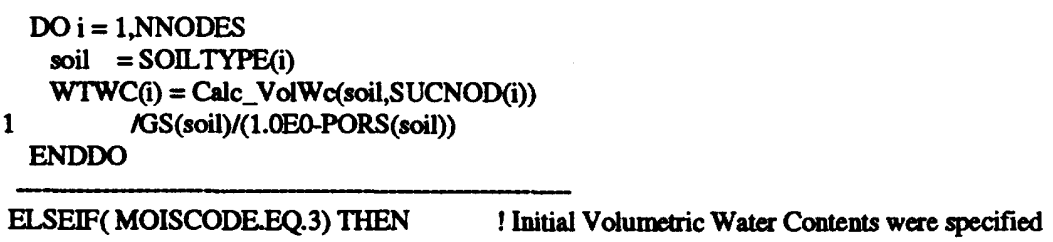

CALL REVERSE_SPLINES ! Reverse the splines order so the it is in ascending suction order DO $\mathrm{i}=1$, MAX_TYPES

CALL WtSplin2(i,PONTS1,XVOLWC,XSUC,SPLINSL1)

ENDDO

DO $\mathrm{i}=1$, NNODES

soil $=$ SOIL TYPE(i)

$2=\exp ($ XVOLWC(POINTS1(soil),soil))

$\mathrm{tx}=$ WTWC(i) I These values are VolWc's already

IF( tx.LT.t2) THEN

SUCNOD(i)=FN_POINT(soil,POINTS1,XVOLWC,XSUC,SPLINSL1,tx)

ELSE

SUCNOD(i)=(SUCT_INT(soil)-tx)/RM2WA(soil)

ENDIF

WTWC(i) $=$ WTWC(i)/(GS(soil)*(1.0E0-PORS(soil))) $!$ Convert VolWc to GravWC

enddo $! i=1$,nnodes

CALL REVERSE_SPLINES ! Re-Establish the original spline order

DO $\mathrm{i}=1$, MAX_TYPES

CALL WtSplin2(i,POINTS1,XSUC,XVOLWC,SPLINSL1)

ENDDO

ENDIF ! IFCMOISCODE..

RETURN

END

C This function changes the head boundary condition of the bottom node

C of the top node from a water content to matric suction.

C

\section{SUBROUTINE VWC_TO_HEAD}

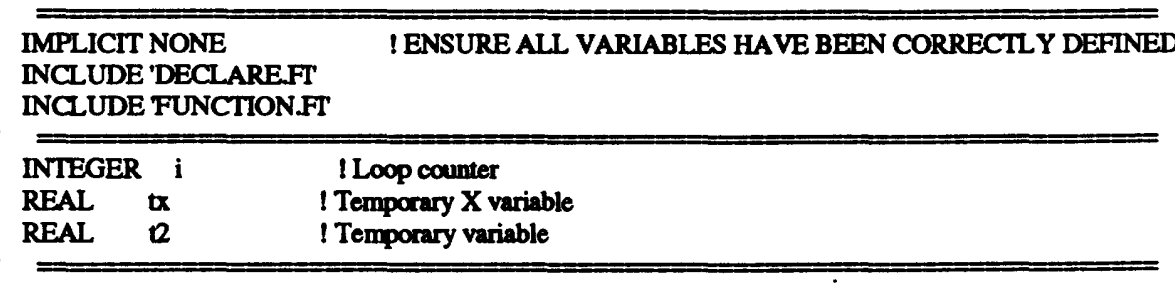

IF(TOP_MOIS_BNDRY(3).OR.BOT_MOIS_BNDRY(3))THEN

CALL REVERSE_SPLINES ! Reverse the splines order so the it is in ascending suction order

DO $i=1$, MAX_TYPES

CALL WtSplin2(i,POINTS1,XVOLWC,XSUC,SPLINSL1)

ENDDO

IF(TOP_MOIS_BNDRY(3).AND.(EBW(1,3).NE.1E10))THEN

t2 $=\exp (x \mathrm{xol} w c($ POINTS1(SOIL TYPE(1)),SOLTYPE(1)))

$\mathrm{tx}=\mathrm{EBW}(1,3)$

if( tx.lt.12) then

EBW $(1,3)=-1$ * fn_point(SOILTYPE(1),POINTS1,xvolwc,xsuc,

else SPLINSL1, tx)

$\operatorname{EBW}(1,3)=-1 *($ suct_int (SOILTYPE(1))-tx)/RM2WA(SOILTYPE(1))

endif

ENDIF

IF(BOT_MOIS_BNDRY(3).AND.(EBW(2,3).NE.1E10))THEN

$0=\exp (x$ volwc(POINTS1(SOIL TYPE(NNODES)),SOIL TYPE(NNODES)))

$\mathrm{tx}=\operatorname{EBW}(2,3)$

if (tx.lt.t2) then 
1

EBW $(2,3)=-1$ * fn_point(SOILTYPE(NNODES),POINTS1,xvolwc,xsuc,

else SPLINSL1, $\mathrm{t}$ )

1

EBW(2,3) $=-1$ * (suct_int(SOILTYPE(NNODES))-tx)

1 endif

ENDIF

CALI REVERSE_SPLINES ! Re-Establish the original spline order

DO $\mathrm{i}=1$,MAX_TYPES

CALL WtSplin2(i,PONTS1,XSUC,XVOLWC,SPLINSL1)

ENDDO

ENDIF

C

RETUR

END

C This subroutine smoothes user supplied points.

Smooth (soil,pnts, $X, Y, 0$ rder,

\section{IMPLICIT NONE I Ensure that all variables have been correctly defined}

INCLUDE 'CONSTANT.FT

C

\begin{tabular}{ll}
\hline integer $\mathrm{i}, \mathrm{j}, \mathrm{t}$ & ! Loop counters \\
integer soil & ! The current layer \\
integer order & ! The order of smoothing required \\
& INTEGER
\end{tabular}

INTEGER pnts (MAX_TYPES) INumber of Data Pts. in VolWc vs Suction

integer times $\quad$ INumber of times to smooth the data

integer type I Type of Smoothing

REAL X (MAX_POINTS,MAX_TYPES) I Suction Data for Suction vs. Perm.

REAL Y (MAX_POINTS,MAX_TYPES) ! Volumetric Water Content for Suct. vs WC.

real ty(max_points)

C

if(type.eq_semi_log)then

do $\mathrm{i}=1$, pnts(soil)

$X(i$, soil $)=\log (X(i$, soil $))$ ! Smooth with Logarithmic X scale

enddo

else if(type.eq.logarithmic)then

do $i=1$,pnts(soil)

$X(1$, soil $)=\log (X(1, s o i))$ ! Smooth with Logarithmic $X$ scale

$Y(i$, soil $)=\log (Y(i$, soil $))$ ! Smooth with Logarithmic $Y$ scale

enddo

endif

C

do $t=1$, times

do $\mathrm{i}=2$, order

ty(i) $=\mathbf{Y}(\mathbf{i}$, soil $)$

do $\mathrm{j}=1, \mathrm{i}-1$

ty(i) $=t y(i)+Y(i-j$, soil $)+(X(i$, soil $)-X(i-j$, soil $))$

$1 *(Y(i+j, s o i l)-Y(i-j, s o i l)) /(X(i+j, s o i l)-X(i-j, s o i l))$

enddo

ty(i) $=\operatorname{ty}(\mathbf{i}) / \mathrm{i}$

enddo

c

do $i=$ (order+1),pnts(soil)-order

ty(i) $=Y(i$, soil $)$

do $j=1$, order

ty(i) $=$ ty(i) $+Y(i-j$, soil $)+(X(i$, soil $)-X(i-j$, soil $))$ *(Y(i+j,soil)-Y(i-j,soil) $) /(X(i+j$, soil $)-X(i-j, s o i l))$

enddo

$\operatorname{ty}(\mathrm{i})=\operatorname{ty}(\mathrm{i}) /(\operatorname{arder}+1)$

enddo

do $i=$ pnts(soil)-order+1,pnts(soil)-1

ty(i) $=Y(i$, soil $)$

do $j=1$,pnts(soil)-i

$\operatorname{ty}(i)=\operatorname{ty}(i)+Y(i-j$, soil $)+(X(i$, soil $)-X(i-j$, soil $))$ 
enddo

ty $(i)=t y(i) /($ pnts $($ soil $)-i+1)$

enddo

c

do $\mathrm{i}=2$,pnts(soil)-1

$Y(i, s o i l)=t y(i)$

enddo

enddo

C

if(type.eq_semi_log)then

do $i=1$,pnts(soil)

$X(i$, soil $)=\exp (X(i$, soil $))$

enddo

else if(type.eq.logarithmic)then

do $\mathrm{i}=1$,pnts(soil)

$\mathbf{X}(i$, soil $)=\exp (X(i, s o i l))$

$Y(i$, soil $)=\exp (Y(i$, soil $))$

enddo

endif

RETURN

END

C

\section{SUBROUTINE SPLINES(Debug_Splines)}

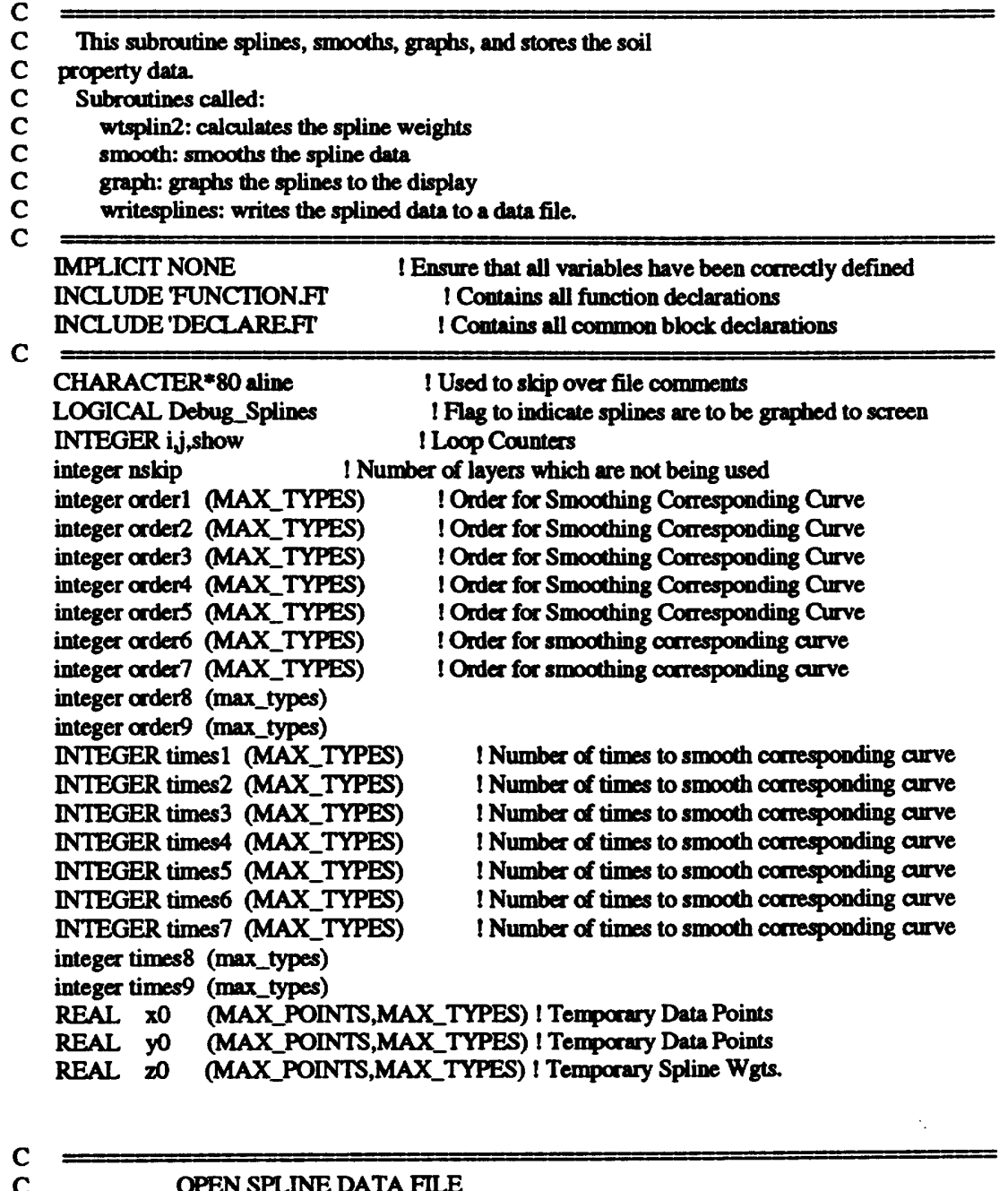




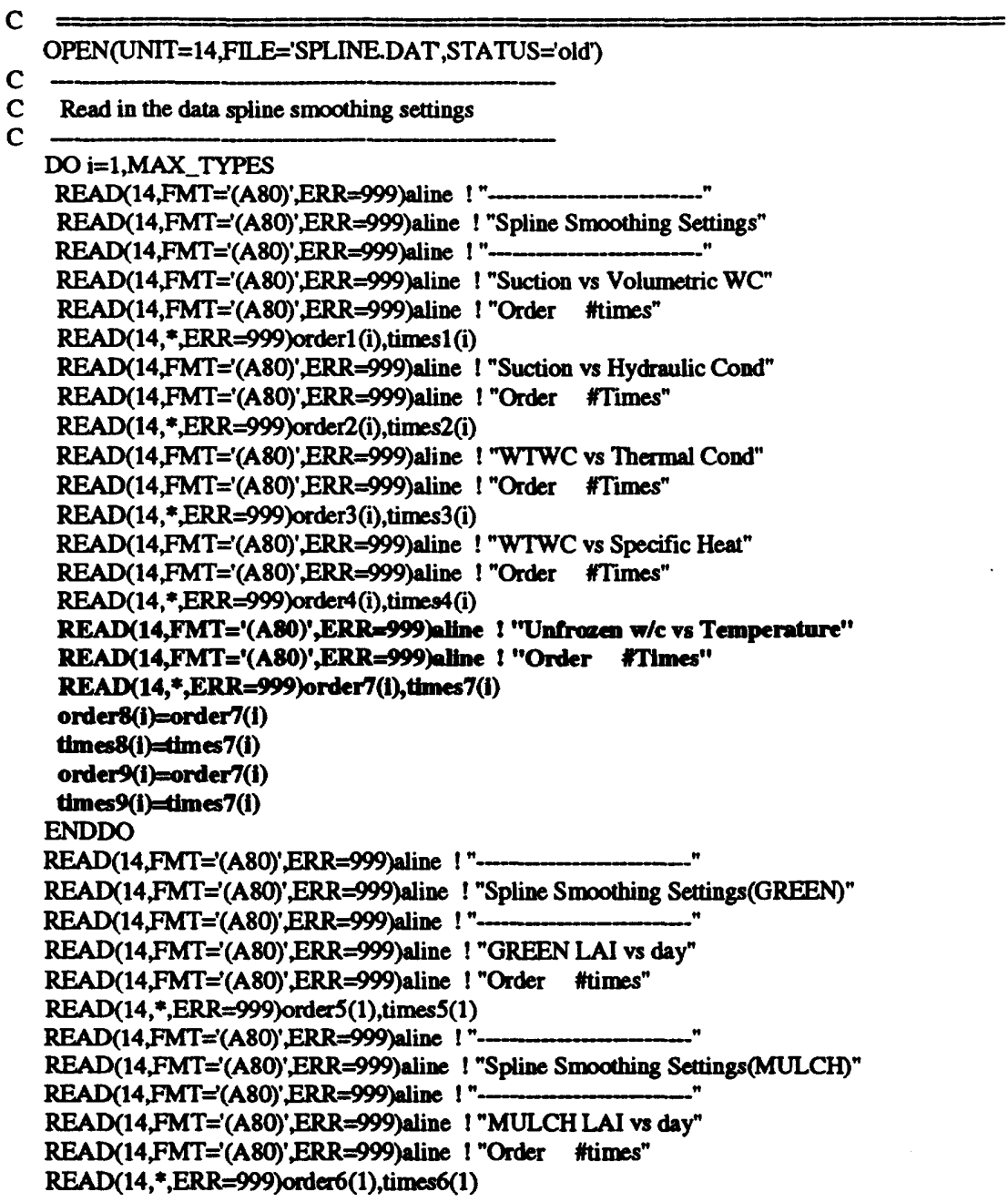


i=show

call graph(i,POINTS1,XSUC,XVOLWC,SPLINSL1,y0,z0,

1 'Suction (kPa)','Volumetric Water Content (dec.)',semi_log) ENDDO

ENDIF

DO $\mathrm{j}=1$, MAX TYPES

Store Smoothed Curves

DO $\mathrm{i}=1$,POINTS $1(\mathrm{j})$

$X V O L W C(i, j)=y O(i, j)$

SPLINSL1 $(\mathrm{i}, \mathrm{j})=\mathbf{2 0}(\mathbf{i}, \mathrm{j})$

ENDDO

ENDDO

C SPLINING OF THE SUCTION VS K DATA

C

DO $\mathrm{i}=1$, MAX_TYPES

CALL WLSplin2(i,POINTS2,XKSUC,XK,SPLINSL2) ENDDO

$$
\text { c }
$$

c

c

DO $j=1$, MAX_TYPES

DO $i=1$, POINTS2 $(j)$

$x O(i, j)=\exp (X K S U C(i, j))$

$y O(i, j)=\exp (X K(i, j))$

ENDDO

CALL SMOOTH(,POINTS2, x0,y0,order2(j),times2(j),logarithmic) ENDDO

DO $\mathrm{i}=1, \mathrm{MAX}$ TYPES

CALL WtSplin2(i,PONNTS2,x0,y0,z0)

ENDDO

IF(Debug_Splines)THEN

c

c DO $\mathrm{i}=1, \mathrm{MAX}$,TYPES

i=show

call graph(i,POINTS2,XKSUC,XK,SPLINSL,2,y0,

1 'Suction (kPa)',Hydraulic Conductivity (cm/s)',logarithmic)

c ENDDO

ENDIF

c

DO j=1,MAX_TYPES

DO $i=1$, POINTS2 $(j)$

$\mathrm{XK}(\mathrm{i}, \mathrm{j}) \quad=\mathrm{yO}(\mathrm{i}, \mathrm{j})$

SPLINSL2 $(\mathrm{i}, \mathrm{j})=\mathbf{z O}(\mathrm{i}, \mathrm{j})$

ENDDO

ENDDO

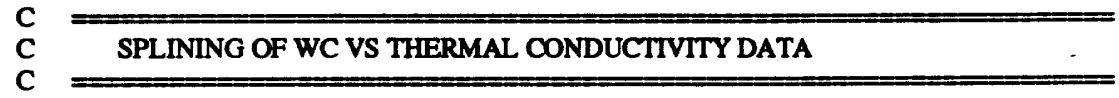

C

DO $\mathrm{i}=1, \mathrm{MAX}$.TYPES

CALL WLSplin2(i,POINTS3,XI_AMDWC,XI_AMD,SPLINSL3) ENDDO

c

DO $\mathrm{j}=1, \mathrm{MAX}$ TYPES

DO $i=1$, POINTS3(j)

$x 0(i, j)=\exp (X \perp A M D W C(i, j))$

$y O(i, j)=\exp (X \perp A M D(i, j))$

ENDDO

CALL SMOOTH(j,POINTS3,x0,y0,order3(j), times3(j),linear)

ENDDO 
DO $i=1, M A X \_T Y P E S$

CALL WtSplin2(i,POINTS3,x0,y0,z0)

ENDDO

IF(Debug_Splines)THEN

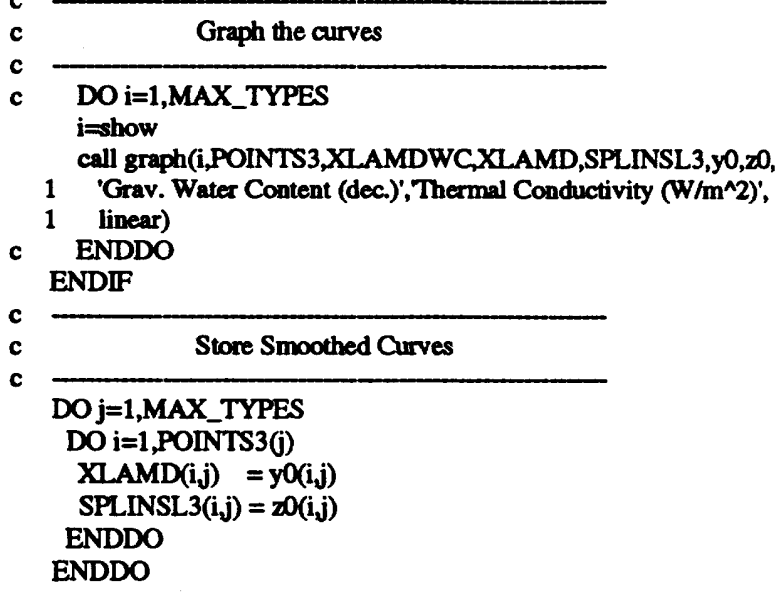

C $=$

C SPLINING OF WC VS VOL SPECIFIC HEAT DATA

C

DO $\mathrm{i}=1$, MAX_TYPES

CALL WtSplin2(i,POINTS4,XSHWC,XSH,SPLINSL4) ENDDO

c

c

DO $\mathrm{j}=1, \mathrm{MAX}$ TYPES

Smooth the curve

DO $i=1$, POINTS4(j)

$x O(i, j)=\exp (X S H W C(i, j))$

$y 0(i, j)=\exp (X S H(i, j))$

ENDDO

CALL SMOOTH(j,POINTS4, $\mathrm{x} 0$,y0,order 4(j), times4(j),linear)

ENDDO

DO $\mathrm{i}=1$, MAX_TYPES

CALL WtSplin2(i,PONTS4,x0,y0,20)

ENDDO

IF(Debug_Splines)THEN

c

c

c DO $\mathrm{i}=1, \mathrm{MAX}$ _TYPES

i=show

call graph(i,POINTS4,XSHWC,XSH,SPLINSL 4,y0,z0,

1 'Grav. Water Content (dec.)';'Specific Heat (J/m^3-C)',

1 linear)

c ENDDO

ENDIF

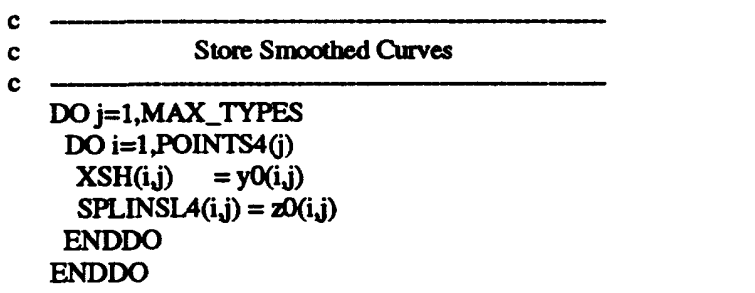

$\mathrm{c}$

C SPLINING OF TEMPERATURE vs UWC

IF(ICE) THEN

DO $\mathrm{l}=1$, MAX_TYPES 
CALL WtSplin2(LPONTS7,XVOLUWC,XTEM,SPLINSL7) ENDDO

c

DO $j=1$, MAX_TYPES

DO $j=1$, POINTS7(j)

$x 0(1, j)=\exp (X V O L U W C(i, j))$

$y O(1, j)=\exp (X T E M(i, j))$

ENDDO

CALL SMOOTH(J,POINTS7,x0,y0,order7(j),times7(j),logarithmic)

ENDDO

DO $:=1$, MAX_TYPES

CALL WiSplin2(1,POINTS7,x0, ,0,20)

ENDDO

IF(Debug_Splines)THEN

c

c

c

\section{Eshow}

Graph the curves

c DO $\mathrm{t}=1, \mathrm{MAX}$ _TYPES

call graph(1,POINTS7,XVOLUWC,XTEM,SPLINSL7,y0,z0,

1 'Unfrozen Water Content (dec.)', 'Negative Temperature (C)',

1 logarithmic)

c ENDDO

ENDIF

c

c

c

Store Smoothed Curves

DO $f=1$, MAX_TYPES

DO $\mathrm{j}=1$, POINTS7()

$X T E M(\mathbf{l}, \mathbf{N})=y 0(\mathbf{L}, \mathbf{l})$

SPLINSL7 $(i, j)=20(i, j)$

ENDDO

ENDDO

ENDIF

C SPLINING OF UWC vs TEMPERATURE

C

IF( ICE) THEN

DO $E 1$, MAX_TYPES

CALL WtSplin2(L,POINTSE, YTEM,YVOLUWC,SPLINSL8) ENDDO

c

c

c

DO $\mathrm{j}=1$,MAX_TYPES

DO $\mathrm{i}=1$, PONTISE(j)

$\operatorname{xO}(\mathbf{W})=\exp (\operatorname{ytem}(\mathbf{W}))$

$\operatorname{yo}(\mathbf{L}, \mathbf{j})=\exp (\operatorname{gvoluw} c(i, j))$

ENDDO

CALL SMOOTH(bOPOIS8, x0,y0,order8(j), times8(j),semi_log)

ENDDO

DO $:=1$, MAX_TYPES

CALL WtSplin2(i,POINTS\&, $0, y 0,=0)$

ENDDO

IF(Debug_Splines)THEN

\section{DO $i=1, M A X \_T Y P E S$}

Eshow

call graph(1,POINTS\&, ytem, yvoluwc,SPLINSL8,y0,z0,

1 'Negative Temperature (C)', 'Unfrozen Water Content (dec)',

1 semi_log)

c ENDDO

ENDIF

c

c

Store Smoothed Curves 
DO $\mathrm{J}=1, \mathrm{MAX}$ _TYPES

DO $i=1$, POINTS\&( $j$ )

yvoluwe $(i, j)=y 0(i, j)$

SPLINSLE $(i, j)=20(i, j)$

ENDDO

ENDDO

ENDIF

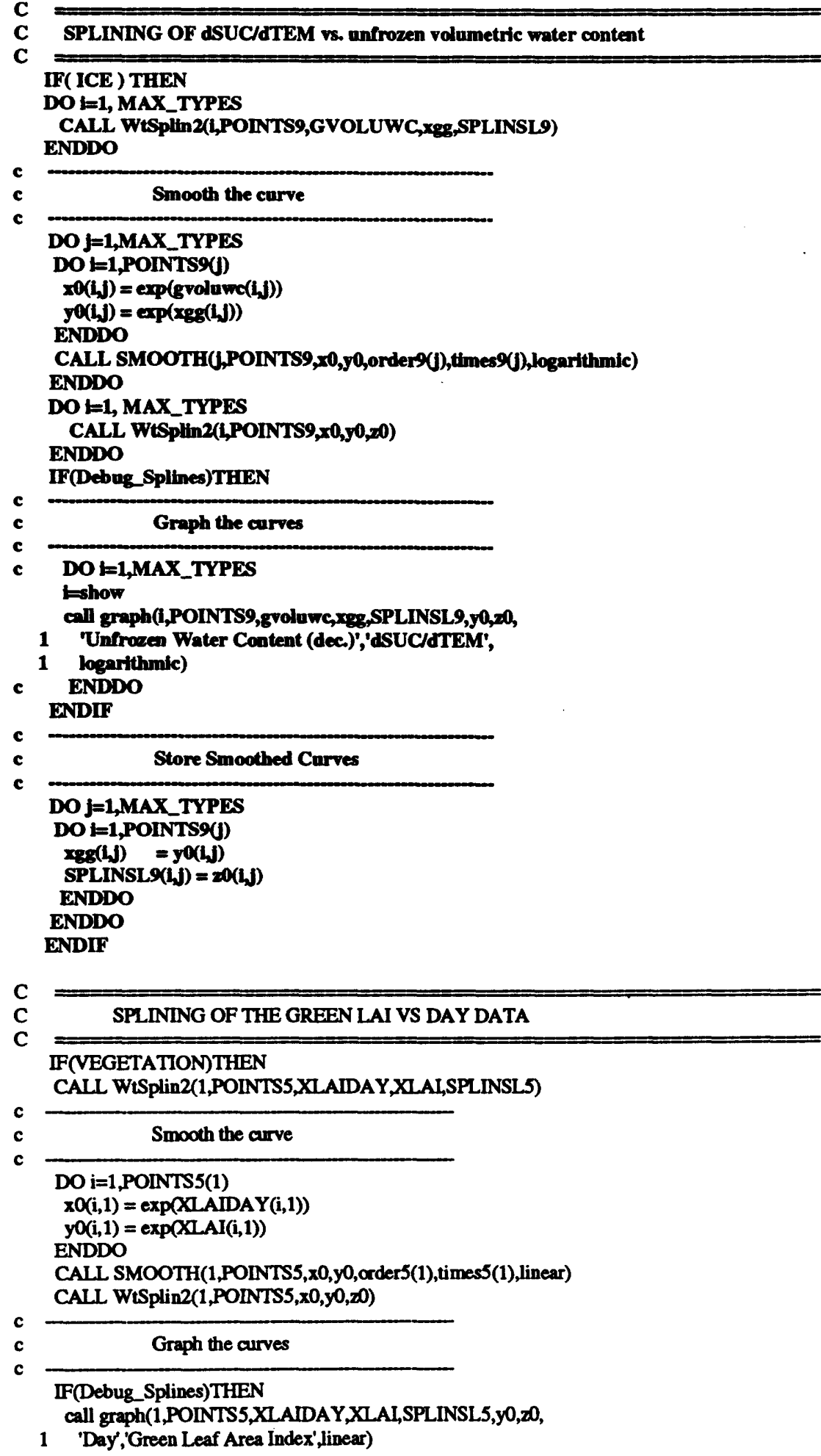




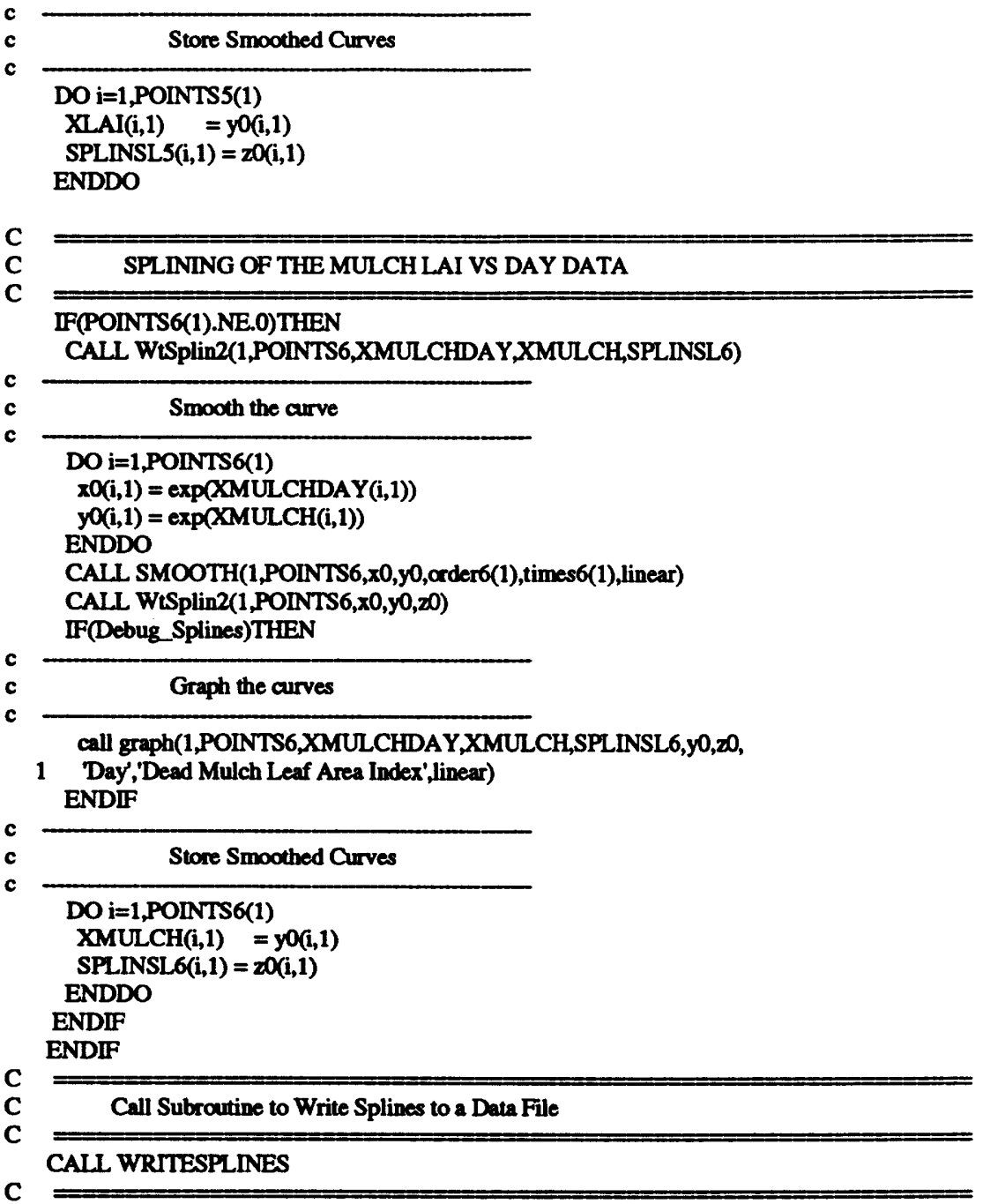

CLOSE(UNIT=14)

RETURN

999 WRITE(*,*) aline

STOP 'Error in splines data file' END

\section{SUBROUTINE WRITE_NOD(Water)}

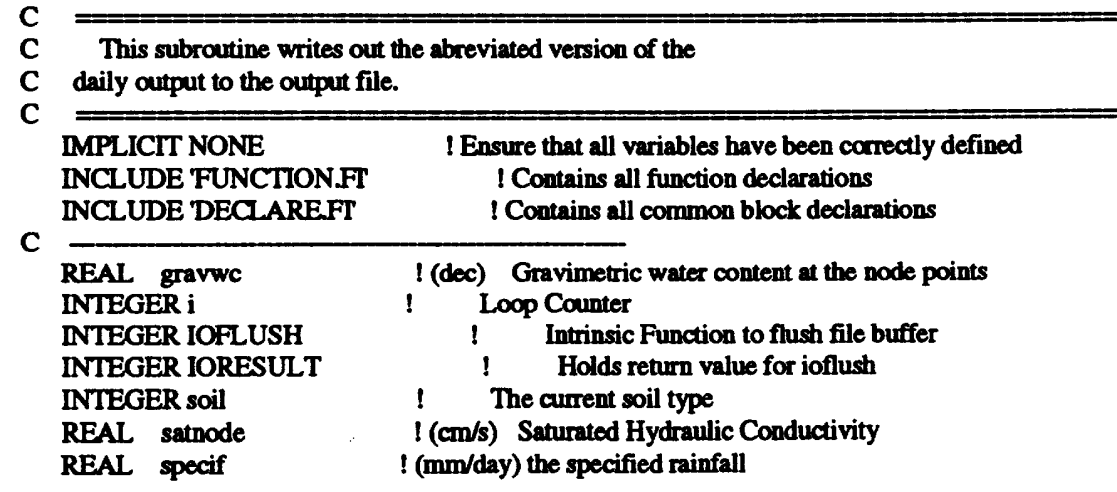


REAL volWatCon

REAL press_head

REAL total_head

REAL avail_poros

REAL Water

REAL k,landa
! (dec) Volumetric Water Content

! ( $(\mathrm{PPa})$ Pressure Head

! (kPa) Total Head

!(\%) Available Porosity

! (mm/day) The Change in Water in the System

$!(\mathrm{cm} / \mathrm{s}),(\mathrm{W} / \mathrm{mC})$

WRTE(48,7)NDAY

write $(48, *)$ time

7 FORMAT( Elpsd time = ',13,' days')

IF( MAXD OUT TODAY.GT.0.0)THEN

WRITE $\left(48,{ }^{*}\right)^{\prime}$ WARNING: The system failed to converge within the '

WRITE(48,*)' specified maximum number of iterations'

WRTTE $\left(48,{ }^{*}\right)^{\prime} \quad$ one or more times during the current day!"

WRITE $(48, *)^{\prime}$ ' Total Non-Covergence Time = ',MAXD_OUT_TODAY

ENDIF

IF(QW(1,2).EQ.1.00E+20)THEN

specif $=0.00 \mathrm{E} 0$

EI SEIF(NDAY.EQ.0)THEN

specif $=0.00 \mathrm{EO}$

ELSE

specif $=Q W(1,2) * 24 * 3600 * 1000$

ENDIF

write $(48,8)$ PEsum

write $(48,9)$ AEsum

write $(48,10)$ PTsum

write $(48,11)$ ATsum

write $(48,12)$ (AEsum + ATsum )

write $(48,13)$ Water

write $(48,14)$ specif

write $(48,15)$ Runoff

IF(VEGETATION)THEN

write $(48,16)$ SFLUX(RootDepth(2))

ELSE

write $(48,17)$ SFL.UX(1)

ENDIF

write $(48,18)$ LAI,MULCH

IF(STEADYSTATE)THEN

WRITE $(*, *)^{*}$ ' Converged'

ELSEIF(.NOT.GRAPHICS)THEN

WRITE $(*, 8)$ PEsum

WRITE $(*, 9)$ AEsum

WRITE $(*, 10)$ PTsum

WRITE $(*, 11)$ ATsum

WRITE $(*, 12)$ (AEsum + ATsum )

WRITE $(*, 13)$ Water

WRITE $(*, 14)$ specif

WRITE $(*, 15)$ Runoff

IF(VEGETATION)THEN

WRITE $(*, 16)$ SFLUX(RootDepth(2))

EISE

WRITE $(*, 17)$ SFLUX(1)

ENDIF

WRITE $(*, 18)$ LAI,MULCH

ENDIF

8 FORMATC Pot. Evap. = ;G9.3,' mm/day ?

9 FORMATC Actual Evap. =',G9.3,' mm/day $)$

10 FORMATC Pot. Transp. =',G9.3, mm/day )

11 FORMATC Actual Transp. = ',G9.3, mm/day )

12 FORMATC Actual Evapotrans. =',G9.3, mm/day )

13 FORMATC Water Balance $=$ ',G9.3,' $\mathrm{mm} /$ day ')

14 FORMATC Specified Rainfall = ',G9.3,' mm/day ?

15 FORMATC Total Runoff = ',G9.3,' mm/day ?)

16 FORMATC Net Infiltr. = ',G9.3,' mm/day (at root base) )

17 FORMATC Net Infiltr. =',G9.3,' mm/day (at soil surface) )

18 FORMATC Leaf AI = ',G9.3;' (Green) ',G9.3,' (Mulch) ')

IF(NDAY.EQ.0)THEN

write(48,*)' Root system extends from n/a to $\mathrm{n} / \mathrm{a} \mathrm{cm}$ depth'

ELSEIF(VEGETATION)THEN

WRITE(48,19) (YCORD(1)-YCORD(RootTop(2))), 


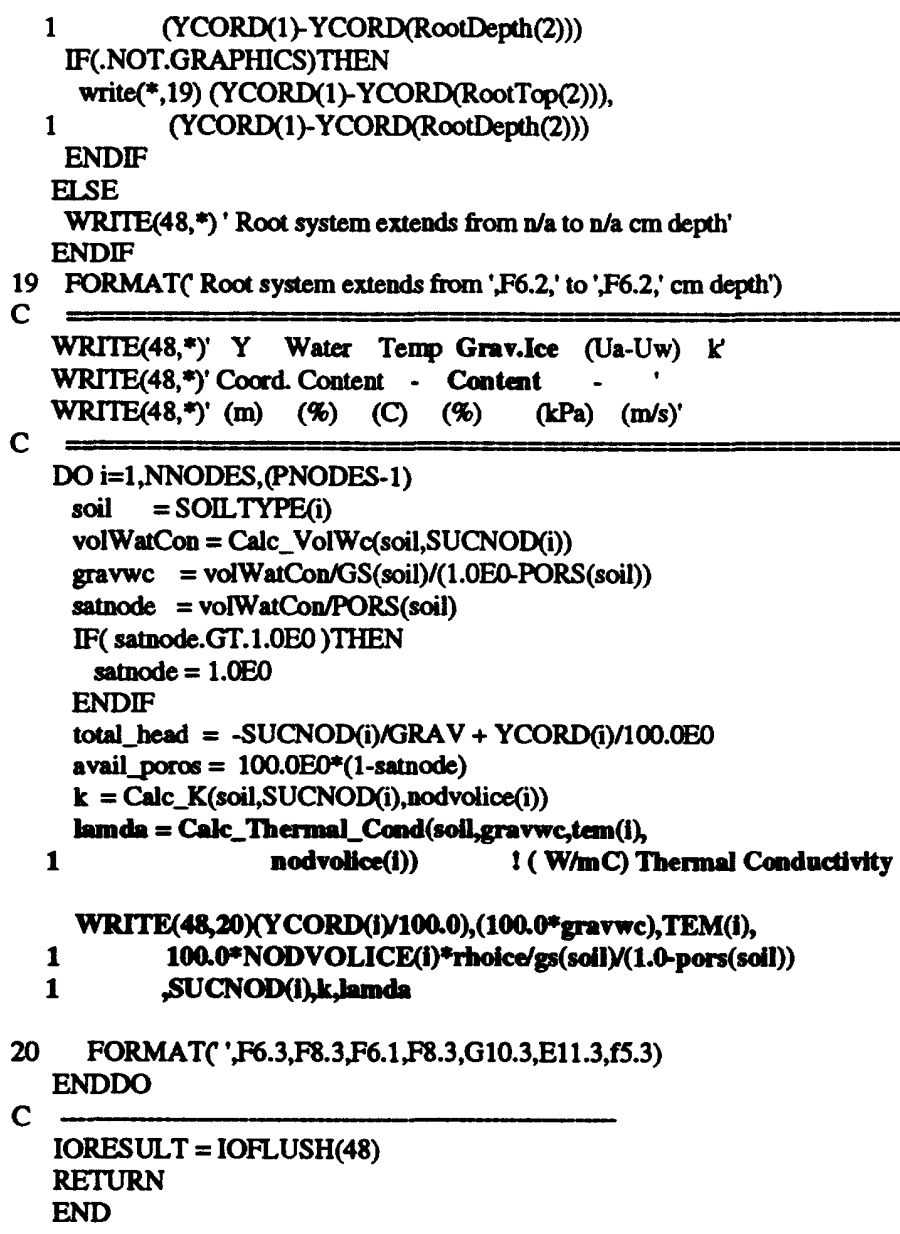

\section{SUBROUTINE WRITE_OUT(Water)}

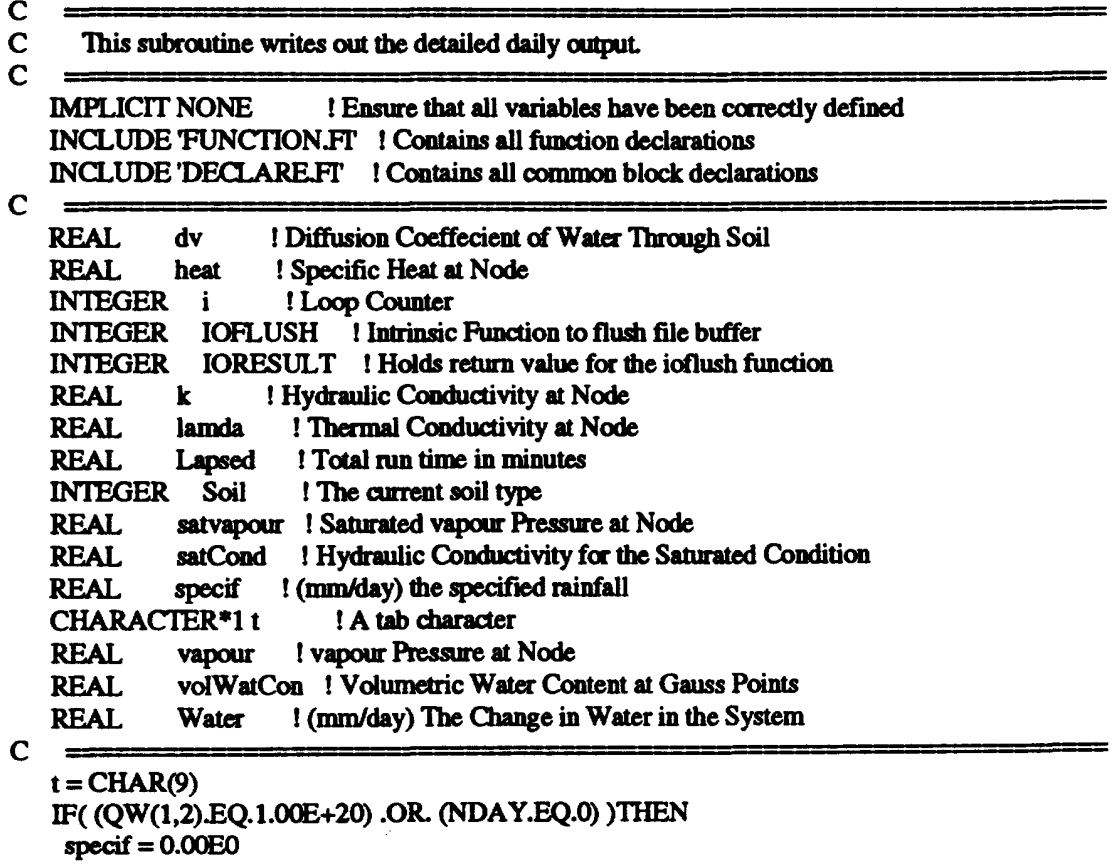


ELSE

specif $=Q W(1,2) * 24 * 3600 * 1000$ ! convert units from m/s to mm/day

ENDIF

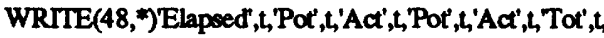

1 Water', L,'Spec',t,'Runoff,t,'LAI, t,'LAT,, ,'Not'

WRITE(48,")'Time',t,'Evap',t,'Evap',t,'Tran',t,'Tran',t,'ET,t,

1 'Bal',L,'Fhux',t,t,'Green',t,'Mulch',t,'Converged'

WRTIE(48,*)'days',t,'(mm)',t,'(mm)',t,'(mm)',, , $\left.^{\prime}(\mathrm{mm})\right)^{\prime, t,}(\mathrm{~mm})^{\prime}, \mathrm{t}$,

$1 \quad$ '(mm)',t,'(mm)',t,'(mm)',t,'(mm)',t,t,'(secnds)'

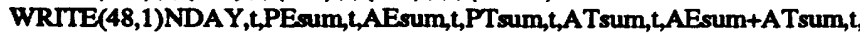

1 Water, L,specif,, Runoff, $, \mathrm{LAI}, \mathrm{L}, \mathrm{MULCH}, \mathrm{t}$

1 MAXD_OUT_TODAY

1 FORMATC ',13,A1,F9.3,A1,F9.3,A1,F9.3,A1,F9.3,A1,F9.3,A1,F9.3,A1,

$1 \quad \mathrm{F9.3,A1,F9.3,A1,G9.3,A1,G9.3,A1,G9.3)}$

IF(STEADYSTATE)THEN

WRITE $(*, *)^{\prime}$ ' Converged'

ELSEIF(.NOT.GRAPHICS)THEN

IF( ((NDAY/22)*22).EQ.NDAY )THEN I Write Headings every 40 days

WRITE(*,*)'DAY PE AE AT SF :

1

- Runoff WB Time'

WRITE $(*, *)^{\prime} \quad(\mathrm{mm}) \quad(\mathrm{mm}) \quad(\mathrm{mm}) \quad(\mathrm{mm})$;

ENDIF

Lapsed $=$ SECNDS(TIME0)/60.0 $!$ Determining the total elapsed time for run so far.

WRITE $(*, 5)$ NDAY,PEsum,AEsum,ATsum,SFLUX(1),Runoff,Water,Lapsed

5

FORMATC ',I3,F8.2,' 'F8.2,' ',F8.2,' ',F8.2,' ',F8.2.

ENDIF

C

WRITE(48,*)'Y,t,'GWC,t,T,t,'Suc',t, TuHd,t,LqF,t,'VpF,t,

1 TtF,t,'PRU',t,ARU,t,'VWC',t,'Sat',t,'HydCnd',t,

1 'Dv',t,' 'VpP

WRITE(48," ')'(m)',t,'(\%)',t,'(C)',t,'(m)',t,'(kPa)',t,'(mm)',t,

$1 \quad$ '(mm)',t,'(mm)',t,'(m/m)',t,'(m/m)',t,'(\%)', t, (\%)',

$1 \quad(\mathrm{~m} / \mathrm{s})^{\prime}, \mathrm{t}, \mathrm{t}^{\prime}(\mathrm{kPa})^{\prime}$

DO $\mathrm{i}=1$, NNODES,(PNODES-1)

soil = SOILTYPE(i)

WTWC(i) $=$ Calc_VolWo(soil,SUCNOD(i))

1 KGS(soil)/(1.0EO-PORS(soil))

volWatCon = WTWC(i)*GS(soil)*(1.0-PORS(soil))

IF( PORS(soil).LT.volWatCon)THEN

satcond $=1.0$

ELSE

satCond $=$ vol WatCon/PORS(soil)

ENDIF

$k=$ Calc_K(soil,SUCNOD(i))

dv = Calc_vapour_Diff(TEM(i)+273.0,volWatCon,NODVOLICE(i),PORS(soil))

satVapour $=$ Calc_SatVp $($ TEM(i) +273.0$)$

IF(SUCNOD(i).It.0.0E0) THEN

vapour $=$ satVapour $* 0.1$

ELSE

vapour $=\exp ((-2.1674 \mathrm{E}-03 * \mathrm{SUCNOD}(\mathrm{i})) /(\mathrm{TEM}(\mathbf{i})+273.0))$

"satVapour*0.1

ENDIF

WRITE $(48,20)$ YCORD(i)/100.0,t,100.0*WTWC(i), t,TEM(i),t,

1 SUCNOD(i),t,YCORD(i)/100.0-SUCNOD(i)/GRAV,t,SFLUXL(i),t,

1 SFLUXV(i),t,SFLUX(i),t,SFLUXPRU(i)/10.,t,SFLUXARU(i)/10.,

$1 \quad t, 100.0^{*}$ volWatCon, $t, 100.0^{*}$ satCond $, t, k, t, d v, t$, vapour

20 FORMATC ',F6.3,A1,F8.3,A1,F6.1,A1,G11.3,A1,G11.3,A1,G10.3,A1,

$1 \mathrm{G} 10.3, \mathrm{~A} 1, \mathrm{G} 10.3, \mathrm{~A} 1, \mathrm{G} 10.3, \mathrm{A1}, \mathrm{G10} .3, \mathrm{~A} 1, \mathrm{~F} 6.2, \mathrm{~A} 1, \mathrm{~F} 8.2, \mathrm{~A} 1$,

1 G9.2,A1,G11.2,A1,F7.2)

ENDDO

C

IORESULT $=$ IOFLUSH(48)

C RETURN

END 


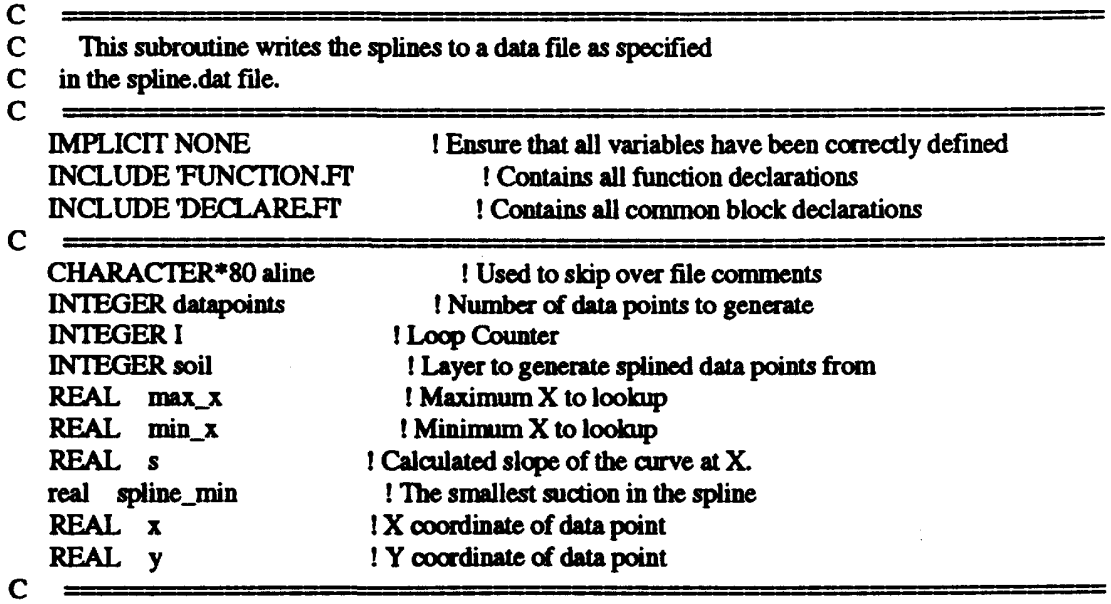

C

READ(14,FMT='(A80)',ERR=999)aline ! " READ (14,FMT='(A80)',ERR=999)aline I"Raw Spline Data Output Sett" READ $(14, F M T=(A 80)$,ERR=999)aline ! "

C $=$ WRITING OF THE SUCTION VS WC DATA

READ(14,FMT='(A80)',ERR=999)aline ! "Suction vs VolWc"

READ(14,FMT='(A80)',ERR=999)aline ! "Layer "Points,Min,Max"

READ(14,")soil,datapoints, $\min \_x, \max \_x$

IF(datapoints.GT.1)THEN

OPEN(UNIT=15,FILE='SUC_WTWC.TXT,STATUS='UNKNOWN')

spline_min $=\exp (\operatorname{xsuc}(1$, soil $))$

DO $20 \mathrm{i}=0$, datapoints -1

$x=\min \_x+\left(\max \_x-\min \_x\right) * I /($ datapoints-1)

IF(X.le.spline_min)THEN

$y=$ (suct_int(soil)-RM2WA(soil)*X)/GS(soil)/(1.0EO-PORS(soil))

$s=$ RM2WA(soil)

ELSE

$y=$ FN_POINT(soil,POINTS1,XSUC,XVOLWC,SPLINSL1, $x$ )

1 NGS(soil)/(1.0EO-PORS(soil))

$s=$ FN_SLOPE(soil,POINTS1,XSUC,XVOLWC,SPLINSL1,x)

ENDIF

WRITE(15,*)x,y,s

20 CONTINUE

CLOSE(UNIT $=15$ )

ENDIF

C

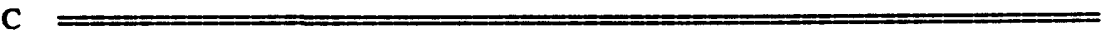

C WRITING OF THE SUCTION VS K DATA

C READ(14,FMT='(A80)',ERR=999)aline ! "Suction vs Hyd Cond"

READ(14,FMT='(A80)',ERR=999)aline I "Layer "Points,Min,Max"

READ(14,*)soil,datapoints,min_x,max_x

IF(datapoints.GT.1)THEN

OPEN(UNIT=15,FILE='SUC_HYD.TXT,STATUS='UNKNOWN')

DO $30 \mathrm{I}=0$, datapoints -1

$x=\min x+(\max x-\min x) * V /($ datapoints-1 $)$

$y=$ FN_POINT(soil,POINTS2,XKSUC,XK,SPLINSL2,

$+\quad \mathrm{X}) / 100.0 \mathrm{EO}$

WRITE $(15, *) x, y$

30 CONTINUE

CLOSE(UNIT $=15$ )

ENDIF 
C READ(14,FMT='(A80)',ERR=999)aline ! "WtWc vs Therm Cond" READ(14,FMT='(A80)',ERR=999)aline ! "Layer \#Points,Min,Max" READ(14,")soil,datapoints,min_x,max_x

IF(datapoints.GT.1)THEN

OPEN(UNIT=15,FIL,E='WTWC_THC.TXT,STATUS='UNKNOWN')

DO $40 \mathrm{I}=0$,datapoints- 1

$x=\min \_x+\left(\max x_{-}-\min \_x\right) * V /($ datapoints-1 $)$

$y=$ FN_POINT(soil,POINTS3,XI_AMDWC,XIAMD,SPLINSL3, $x$ )

WRITE $\left(15,{ }^{*}\right) x, y$

40 CONTINUE

CLOSE(UNIT=15)

ENDIF

C

$\mathrm{C} \Longrightarrow$

C WRITING OF WC VS VOL SPECIFIC HEAT DATA

READ(14,FMT='(A80)',ERR=999)aline ! "WtWc vs Spec. Heat"

READ(14,FMT="(A80)',ERR=999)aline ! "Layer \#Points,Min,Max"

READ(14,")soil,datapoints,min_x,max_x

IF(datapoints.GT.1)THEN

OPEN(UNIT=15,FILE='WTWC_SPH.TXT,STATUS='UNKNOWN')

DO $50 \mathrm{I}=0$,datapoints- 1

$x=\min \_x+\left(\max \_x-\min \_x\right) * \mathrm{~L} /($ datapoints-1 $)$

$y=$ FN_PONTT(soil,POINTS4,XSHWC,XSH,SPLINSL4, $x$ )

WRITE(15,")x,y

50 CONTINUE

CLOSE(UNIT $=15$ )

ENDIF

C

C WRITING OF TEMPERATURE VE UWC DATA

READ(14,FMT='(A80)',ERR=999)aline ! "Temperature vs Unfrozen Vol w/c"

READ(14,FMT='(A80)',ERR=999)alline ! "Layer "Points,Min,Max"

READ(14, *)eoll,datapoints,min_x,max $x$

IF(datapoints.GT.1)THEN

OPEN(UNIT=15,FILE='TEM_UWC.TXT,STATUS='UNKNOWN')

DO 55 I = 0,datapoints-1

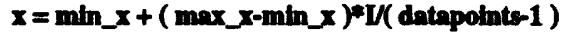

$y=$ FN_POINT(sol,POINTS7,XVOLUWC,XTEM,SPLINSL7, $x$ )

WRTEL $(15, *) x, y$

55 CONTINUE

CLOSE(UNIT=15)

ENDIF

C

C

C WRITING OF UWC VS TEMPERATURE DATA

READ(14,FMT='(A80)',ERR=999)aline ! "Unfrozen Vol w/c vs Temperature"

READ(14,FMT ='(A80)',ERR=999)alime ! "Layer "Points,Min,Max"

READ(14,*)soll,datapoints,min_x,max, $x$

IF(datapoints.GT.1)THEN

OPEN(UNTT=15,FILE='UWC_TEM.TXT',STATUS=UNKNOWN')

DO $56 \mathrm{I}=0$,datopoints-1

$x=\min \_x+\left(\max _{-} x-\min \_x\right) * I /($ datapoints-1 $)$

y = FN POINT(soll,POINTS\&, ytem,yvoluwc,SPLINSL\&,

WRTTE $(15, *) x, y$

56 CONTINUE

CLOSE(UNIT $=15$ )

ENDIF

$\mathrm{C} \Longrightarrow$

C WRITING OF dSUC/dTEM VS UWC DATA

C

READ(14,FMT ='(A80)',ERR=999)aline ! "dSUC/dTEM rs. UWC"

READ(14,FMT='(A80)',ERR=999) aline ! "Layer "Points,Min,Max" 
READ(14,*)soil,datapoints,min_x,max_x

IF(datapoints.GT.1)THEN

OPEN(UNIT=15,FILE='dSdT_UWC.TXT',STATUS='UNKNOWN')

DO 58 I = 0, datapoints-1

$x=\min \_x+\left(\max \_x-\min \_x\right) * I /$ datapoints-1 $)$

$y=$ FN_POINT(soll,POINTS9,gvoluwc,xgg_SPLINSL9,x)

WRITE $(15, *) x, y$

58 CONTINUE

CLOSE(UNIT $=15$ )

ENDIF

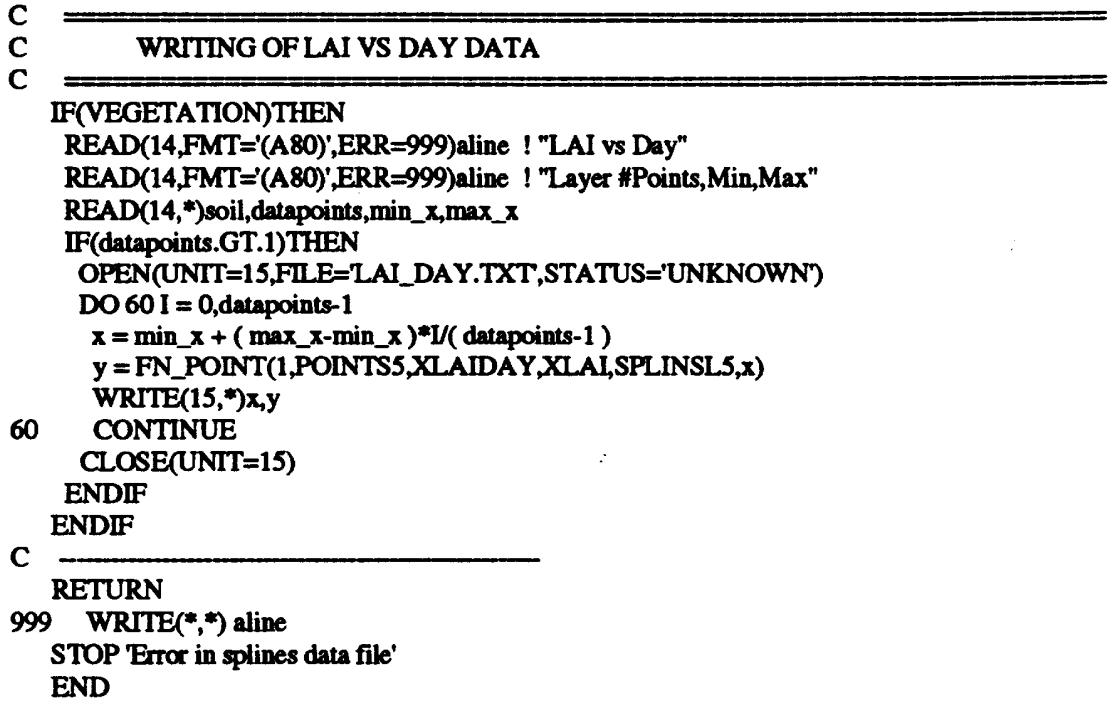

\section{SUBROUTINE WtSplin2(soil,N,X,Y,SPLINE)}

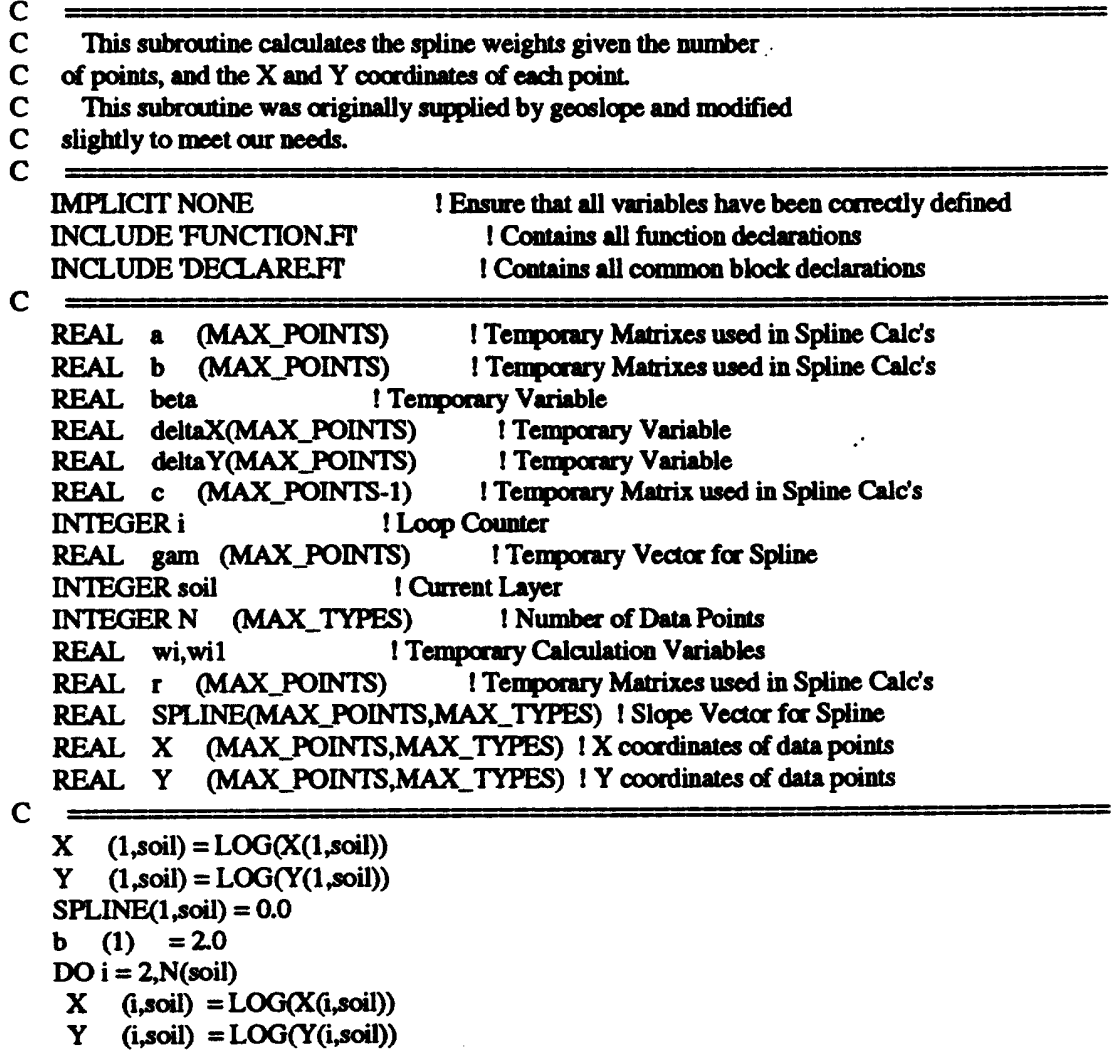


IF ( X(i,soil).LT.X(i-1, soil) )THEN

WRTE $(*, *)$ ' $X$ values not in ascending order '

stop

ENDIF

SPLINE(i,soil) $=0.0$

b (i) $=2.0$

$\operatorname{deltaX}(\mathrm{i}-1)=X(\mathrm{i}$, soil $)-X(\mathrm{i}-1$, soil $)$

$\operatorname{deltaY}(\mathbf{i}-1)=Y(i$, soil $)-Y(i-1$, soil $)$

ENDDO

(1) $=1.0$

$\mathrm{a}(\mathrm{N}($ soil) $)=1.0$

$\mathrm{r}(1)=3.0^{*}(\operatorname{deltaY}(1) \quad / \operatorname{deltaX}(1)$ )

$\mathbf{r}(\mathbf{N}($ soil $))=3.0^{*}($ deltaY $(\mathbf{N}($ soil $)-1) /$ deltaX $(\mathbf{N}($ soil $)-1))$

wil $=\operatorname{EXP}(-3.0 * \operatorname{LOG}(1.0+(\operatorname{deltaY}(1) * \operatorname{deltaY}(1))$

$1 \quad /(\operatorname{deltaX}(1) * \operatorname{deltaX}(1)))$ )

DO $\mathrm{i}=2, \mathrm{~N}$ (soil)-1

wi $=$ wil

wil $=\operatorname{EXP}(-3.0 * \operatorname{LOG}(1.0+(\operatorname{deltaY}(\mathrm{i}) * \operatorname{deltaY}(\mathrm{i}))$

1

$a(i)=w^{*}{ }^{*} \operatorname{deltaX}(i) /\left(w i^{*} \operatorname{deltaX}(i)+w i 1^{*} \operatorname{deltaX}(i-1)\right)$

$c(i)=1.0-a(i)$

$r(i)=3.0 * a(i) *$ delta $Y(i-1) / d e l t a X(i-1)$

1 $+3.0 * c(i) *$ deltaY(i) /deltaX(i)

ENDDO

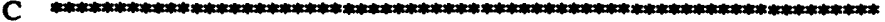

C TRIDIAG_SOLVER

C

IF (ABS(b(1)).LT.1.0E-15) THEN

WRITE $(*, *)$ ' Zero on diagonal; cannot solve Tridiag equations'

stop

END IF

beta $=b(1)$

spline $(1$,soil $)=r(1)$ beta

DO $\mathrm{i}=2, \mathrm{~N}$ (soil)

$\operatorname{gam}(i)=c(i-1)$ beta

beta $=b(i)-a(i) * \operatorname{gam}(i)$

IF ( ABS(beta).LT.1.0E-8) THEN

WRITE(*,*)' Divide by zero; cannot solve Tridiag equations'

stop

END IF

spline $(\mathrm{i}$, soil $)=(\mathrm{r}(\mathrm{i})-\mathrm{a}(\mathrm{i})$ *spline $(\mathrm{i}-1$, soil $)) /$ beta

ENDDO

DO i=n(soil)-1,1, -1

spline $(1$, soil $)=$ spline $(1$, soil $)-$ gam $(i+1) *$ spline $(i+1$, soil $)$

ENDDO

C

DO $\mathrm{j}=1, \mathrm{~N}$ (soil)

IF( $($ (i).EQ.0.0) SPLINE(i,soil $)=0.0 E 0$

ENDDO

C $=$

RETURN

END 


\section{APPENDIX E SUB FUNCTION CODE}


C This function calculates a sin distribution for relative humidity

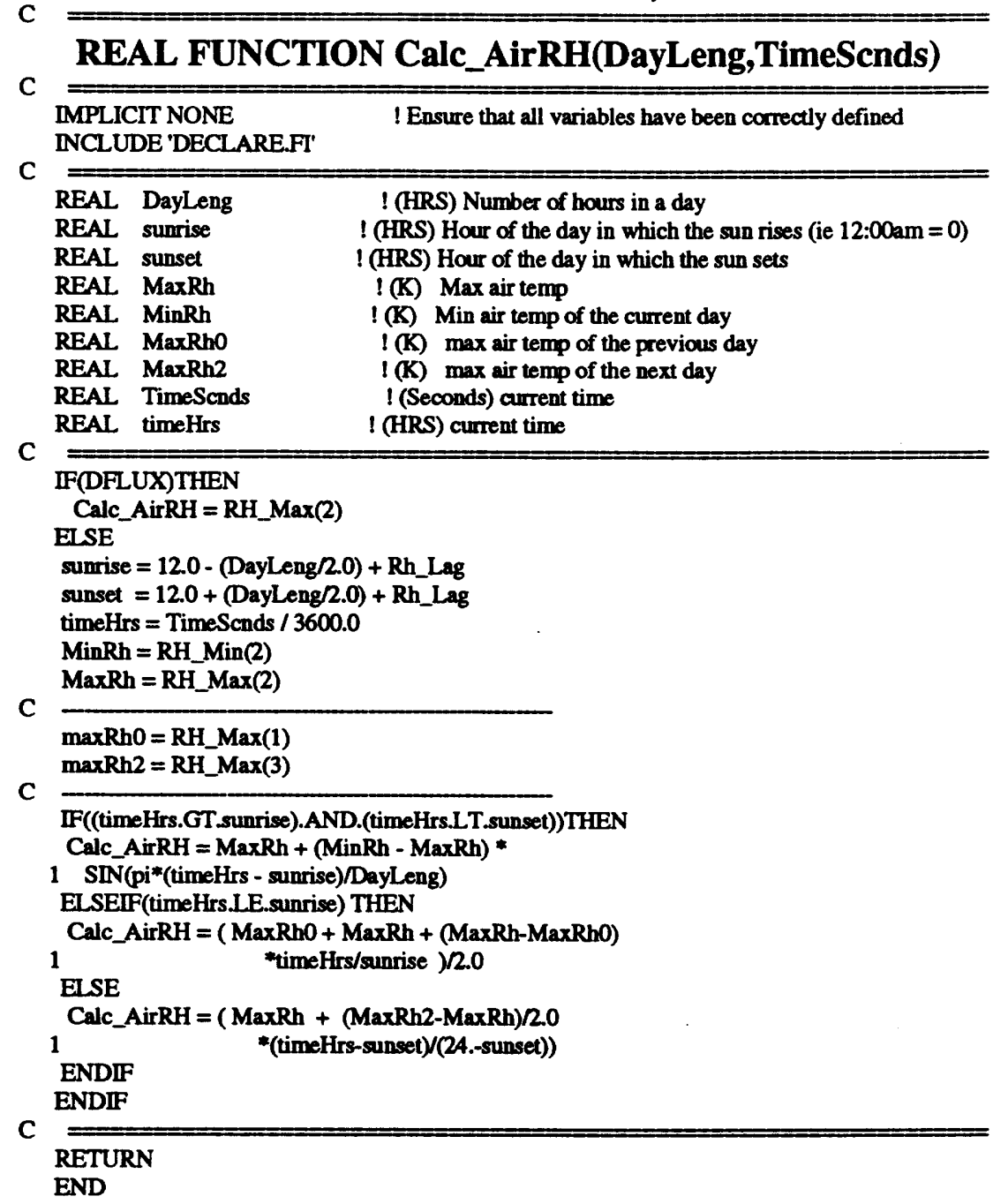

C This function calculates a sin distribution for temperature

c $\overline{\text { REAL FUNCTION Calc_AIRTemp(DayLeng,TimeScnds) }}$

C

IMPLICIT NONE I Ensure that all variables have been correctly defined INCLUDE 'DECLARE.FT'

C REAL Dayleng ! (HRS) Number of hours in a day

REAL max_1 ! (K) Max air temp

REAL min_1 ! (K) Min air temp of the current day

REAL min_0 ! (K) Min air temp of the previous day

REAL min_2 ! (K) Min air temp of the next day

REAL sunrise ! (HRS) Hour of the day in which the sun rises (ie 12:00am =0)

REAL sunset I (HRS) Hour of the day in which the sun sets

REAL TimeScnds ! (scnds) current time

REAL timehrs ! (HRS) current time

C $\overline{\text { IF(DFLUX)THEN }}$

Calc_airtemp $=$ TEMPAMAX $(2)+273.0$

ELSE 


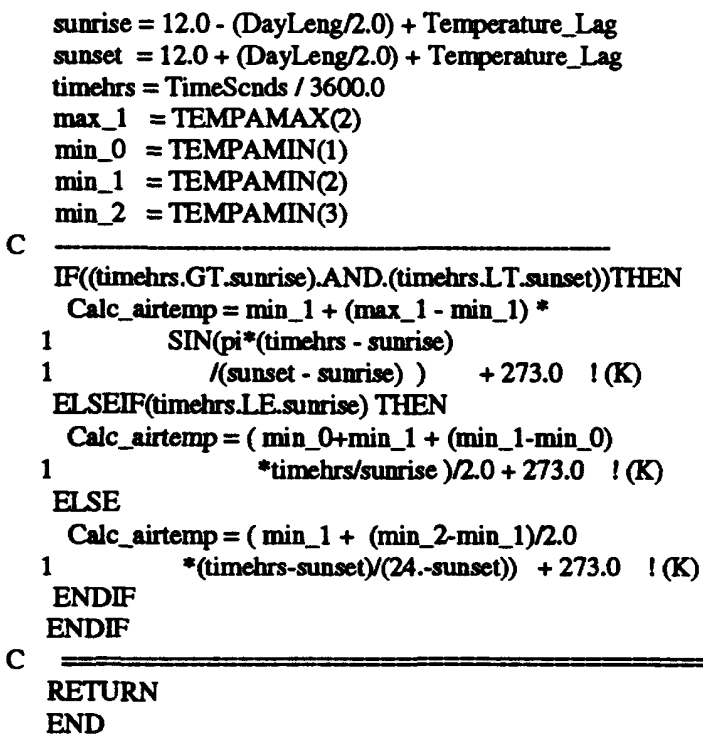

C This function is used by the subroutine TTERATE' to determine

C if convergence has been achieved.

$\mathrm{C}=$

\section{LOGICAL FUNCTION Convergence()} IMPLICIT NONE INCLUDE DECLARE.FT ! Contains all common block declarations

\begin{tabular}{|c|c|}
\hline $\begin{array}{l}\text { INTEGER i,soil } \\
\text { LOGICAL converged } \\
\text { REAL diff } \\
\text { REAL volwc,voluwc } \\
\text { REAL fn_point }\end{array}$ & $\begin{array}{l}\text { ! Loop Counter } \\
\text { I Logical flag to indicate when system has converged } \\
\text { ! Relative change in Suction or Temperature }\end{array}$ \\
\hline
\end{tabular}

C

converged $=$ TRUE

$\mathrm{i}=0$

DO WHIILE(i.LT.NNODES .AND. converged)

$\mathbf{i}=\mathbf{i}+\mathbf{1}$

C

C Convergence for suction is based upon a relative convergence

C which is checked at every node.

C

IF(PRESNOD(i).NE.0.0E0)THEN I Prevent division by zero diff =ABS ( SUCNOD(i)-PRESNOD(i) )/PRESNOD(i)

ELSEIF(SUCNOD(i).NE.0.0EO)THEN diff $=1.0 E 0$

ENDIF

IF(diff.GT.PUSNORM)THEN

converged = FALSE

IF( STEADYSTATE)THEN

WRITE $(*, 1)$ i,diff*100.0,SUCNOD(i)

1 FORMATC Node',I3,' Change ',F6.2,

1 '\% Suction',G17.4)

ENDIF

ENDIF

C Convergence for temperature is based upon a relative conv.

C which is checked at every node.

C IF(PRETNOD(i).NE.0.0E0)THEN !Prevent division by zero diff = ABS( TEM(i)-PRETNOD(i) )/PRETNOD(i) 


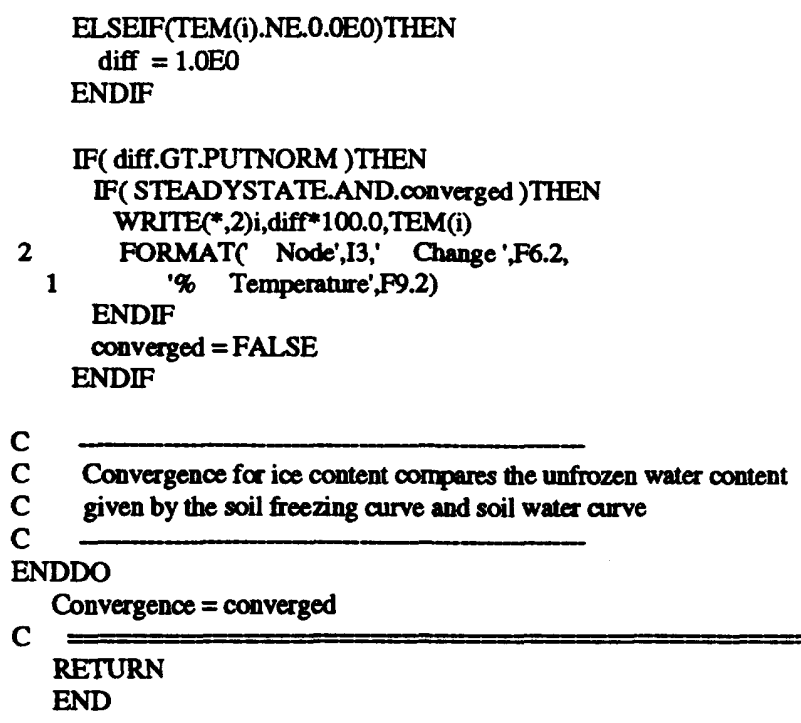

C This function calculates the time (HR) of the DAYLENG
$\mathrm{C}=$

\section{REAL FUNCTION Calc_DAYLENG(N) !(Hours)}
$\mathrm{C}=$ INCLUDE 'DECLARE.FT'
$\mathrm{C}=$
REAL degrees ! Degrees
REAL ws ! Daylight angle (degrees)
INTEGER N ! No. of days past Jan 1.
C
degrees $=23.45 * \operatorname{SIN}((360.0 *(284.0+\mathrm{N}) / 365) * \mathrm{PI} / 180)$
ws $=$ ACOS $(-$ TAN(LAT*PI/180.0)*TAN(degrees*PI/180.0))
Calc_DAYLENG $=2 *(12.0 \mathrm{PI}) *$ ws
C RETURN
END

C This function does a spline lookup and returns the $Y$ value

C corresponding to the supplied $X$ value.

c

REAL FUNCTION Fn_Point(Type,N,X,Y,Spline,Lookup)

C I Ensure that all variables have been correctly defined INCLUDE DECLAREFT I Contains all common block declarations

C INTEGER Type I Current Layer

INTEGER N (MAX_TYPES) ! Number of Points in Spline

REAL X (MAX_POINTS,MAX_TYPES) IX coordinates of data points

REAL Lookup IX value of point to loolap

REAL Y (MAX_POINTS,MAX_TYPES) ! Y coordinates of data points

REAL Spline(MAX__POINTS,MAX

C

$\begin{array}{ll}\text { REAL a,b,hi,phi,xi } & \text { ! Temporary Calculation Vars } \\ \text { INTEGER } \mathbf{k} \text {,khi,klo } & \text { ! Temporary Calculation Vars } \\ \text { REAL newX } & \text { ! Log of X value of point to lookup } \\ \text { REAL newY } & \text { ! Y value at newX }\end{array}$

C

newX = LOG(Lookup)

IF ( newX.LT.X(1,Type) ) THEN

Fn_Point $=\operatorname{EXP}(1$, Type $))$

RETURN 
END IF

IF ( newX.GT.X(N(Type),Type) ) THEN

Fn_Point $=$ EXP(Y (N(Type),Type))

RETURN

C

END IF

C Find the two points the value lies between ( binary search )

C

$\mathbf{k l o}=1$

khi $=$ N(Type)

do while( (khi-klo).GT.1 )

$\mathbf{k}=(\mathbf{k h i}+\mathbf{k l o}) / 2$

if (X(k,Type).GT.newX)THEN

khi $=k$

else

llo $=k$

endif

enddo

C

C Calculate the lookup point

C

$a=$ newX $\quad-X(k l o, T y p e)$

b = newX $\quad$-X(khi,Type)

hi $=X($ (hi, Type) $-X(k l o, T y p e)$

phi $=2.0 /\left(\mathrm{hi}^{* * 3}\right)^{*}\left(\mathrm{a}+0.5^{*} \mathrm{hi}\right)^{*}\left(\mathrm{~b}^{* * 2}\right)$

$x \mathrm{i}=1.0 /\left(\mathrm{hi}^{* * 2}\right) *(\mathrm{a} \quad)^{*}(\mathrm{~b} * * 2)$

new Y $=$ Y(klo,Type)*phi + Spline(klo,Type)*xi

phi $=-2.0 /\left(h^{* * * 3}\right) *(b-0.5 * h i) *\left(a^{* *} 2\right)$

$\mathrm{xi}=1.0 /\left(\mathrm{hi}^{* * 2}\right) *(\mathrm{~b} \quad) *(\mathrm{a} * * 2)$

newY = newY + Y(khi,Type)*phi + Spline(khi,Type)*xi

Fn_Point $=\operatorname{EXP}($ newY $)$

RETURN

END

C This function does a spline lookup and returns the siope of

C the function at the supplied $X$ value.

$\mathrm{C}=$

REAL FUNCTION Fn_Slope(Type,N,X,Y,Spline,Lookup)

IMPLICIT NONE
INCLUDE DECLAREFT
$\begin{aligned} & \text { IEnsure that all variables have been correctly defined } \\ & \text { IContains all common block declarations }\end{aligned}$

C

INTEGER Type !Current Layer

INTEGER N (MAX_TYPES) ! Number of Points in Spline

REAL X (MAX_POINTS,MAX_TYPES) IX cocrdinates of data points

REAL Lookup IX value of point to looku

REAL Y (MAX_POINTS,MAX_TYPES) I Y coordinates of data points

REAL Spline(MAX_POINTS,MAX_TYPES) ICalculated data from Spline

REAL a,b,hi,phi,xi ITemporary Calculation Vars

INTEGER k,khi,klo ! Temporary Calculation Vars

REAL newX

REAL nEWY

! $\mathrm{Log}$ of $\mathrm{X}$ value of point to lookup

REAL slope

I $Y$ value corresponding to the provided $X$

I Slope value at newX

C Function Name Declarations

REAL Fn_Point

! Function which calculates the $Y$ value of the Spline at a specified point

$\mathrm{C}$

newX $=$ LOG(Lookup) 


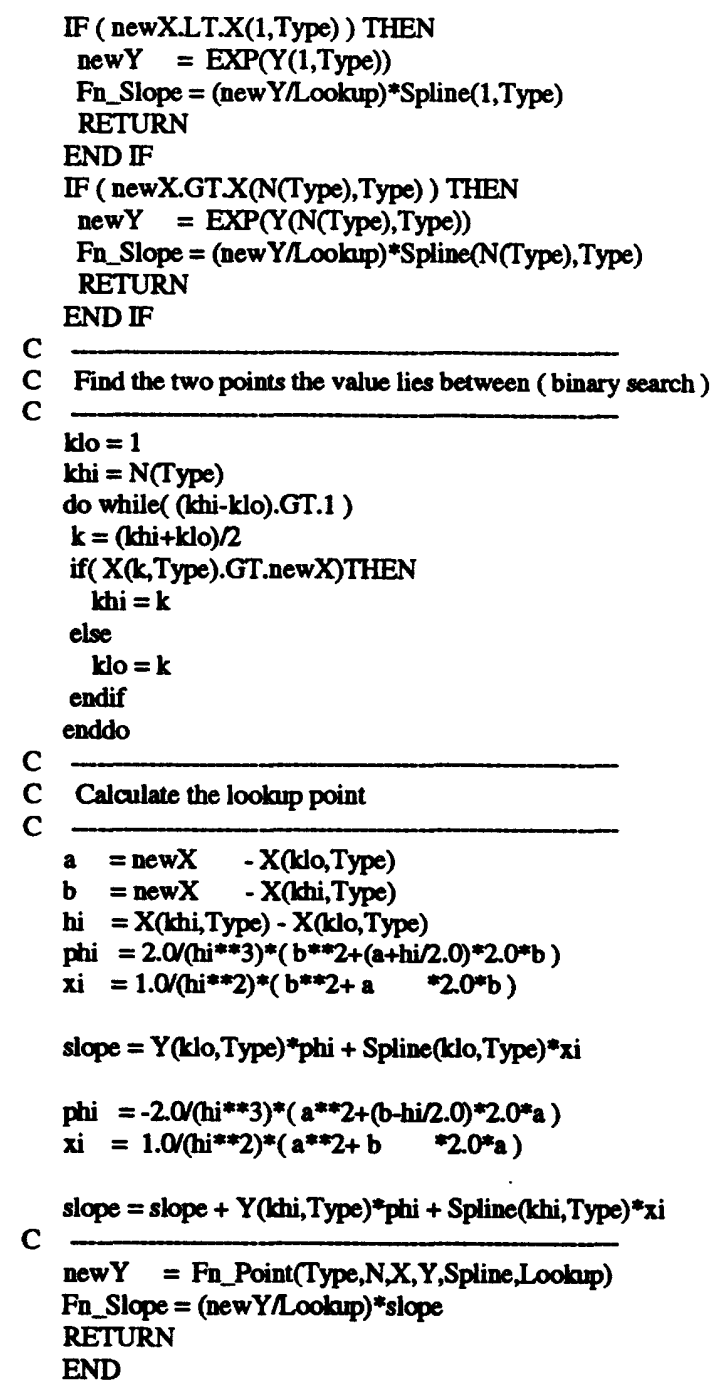

C

REAL FUNCTION

Calc_gFlux(Type,E,C,Sc_0,Sc_1,Pv_0,Pv_1,Gtemp,Gcord,gvolice)

C This subroutine computes the liquid and vapour fluxes at the

C element boundaries.

C $=$

INCLUDE 'DECLARE.FT' ! All include files in here INCLUDE 'FUNCTION.FT I All function defined here

C

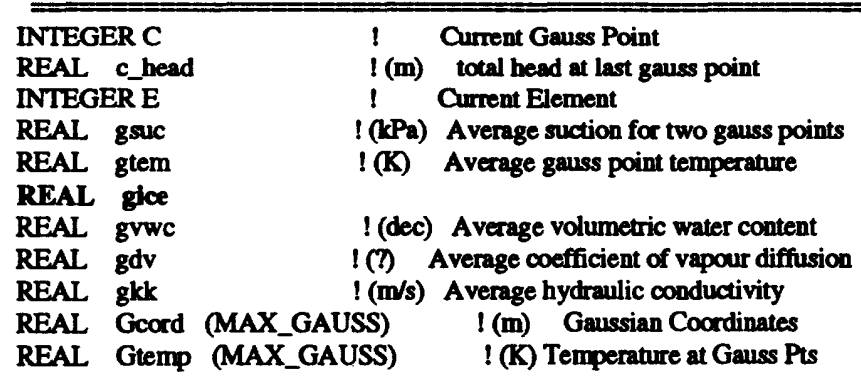


REAL Gvolice (MAX_GAUSS)

$\begin{array}{ll}\text { INTEGER 1 } & ! \text { Last Gauss Point } \\ \text { REAL 1_gradient } & !(\mathrm{m} / \mathrm{m}) \text { Head Gradient } \\ \text { REAL 1_head } & !(\mathrm{m}) \text { total head at last gauss point } \\ \text { INTEGER Type } & ! \text { Current Soil Type } \\ \text { REAL Pv_0 } & !(\mathrm{kPa}) \text { Vapour pressure at last gauss point } \\ \text { REAL Pv_1 } & !(\mathrm{kPa}) \text { Vapour pressure at current gauss point } \\ \text { REAL Sc_0 } & !(\mathrm{kPa}) \text { Suction at last gauss point at the half time step } \\ \text { REAL Sc_1 } & !(\mathrm{kPa}) \text { Suction at current gauss point at the half time step } \\ \text { REAL v_gradient } & !(\mathrm{kPa} / \mathrm{m}) \text { Vapour Pressure Gradient }\end{array}$

C

IF( C.EQ.AGAUSS .OR. (E.EQ.1 .AND. C.EQ.2) )THEN

$1=\mathrm{C}-1$

gsuc $=\left(S c \_0+S c \_1\right) / 2.0$

gtem $=($ gtemp $(1)+$ gtemp $(C)) / 2.0$

gice $=($ grolice(l) + gvolice $(c)) / 2.0$

1_head $=$ gcord(I) - Sc_ONGRAV

c_head $=\operatorname{gcord}(C)-S c \_1 /$ GRAV

I(m) Suction

! (K) Average gaussian temperature

I(m) total head @ m

1 gradient $=(1$ head-c_head $) /(\operatorname{gcord}(\mathrm{l})$-goord $(\mathrm{C})) !(\mathrm{m} / \mathrm{m})$ total head gradient

gkk = Calc_K(Type,gsuc) $\quad !(\mathrm{m} / \mathrm{s})$ Hydraulic Conductivity @ $\mathrm{t}+\mathrm{dt} / 2$

v_gradient $=\left(\mathrm{Pv}_{-} \mathrm{O}-\mathrm{Pv} \_1\right) /(\operatorname{gcord}(\mathrm{l})$-gcord(C) $) \quad I(\mathrm{kPa} / \mathrm{m})$ vapour pressure gradient

gvwc = Calc_VolWc(Type,gsuc) $!(\mathrm{dec})$ Volumetric Water Content

gdv =Calc_Vapour_Diff(gtem,gvwc,gice,PORS(Type)) ! (m/s) Coefficient of Vapour Diffusion

IF(C.EQ.2 AND. E.EQ.1)THEN ! If first element \& 2nd gauss pt.

FLUX_L(1) $=$ gkk * l_gradient * $1000.0 \quad !(\mathrm{mm} / \mathrm{s})$ liquid flux

FLUX_V(1) $=g d v * v_{\text {gradient }} * 1000.0 \quad !(\mathrm{mm} / \mathrm{s})$ vapour flux

VFLUX (1) = FLUX_L(1) + FLUX_V(1) ! $\quad$ (mm/s) total flux @ surface

ENDIF

IF(C.EQ.AGAUSS)THEN ! If last gauss pt. of element

FLUX_L(E+1)=gkk * I_gradient * $1000.0 \quad !(\mathrm{mm} / \mathrm{s})$ liquid flux

FLUX_V $(E+1)=g d v * v_{\text {gradient }} * 1000.0 \quad !(\mathrm{mm} / \mathrm{s})$ vapour flux

Calc_gFlux $=\left(F L U X \_V(E+1)+F L U X \_L(E+1)\right) \quad !(\mathrm{mm} / \mathrm{s})$ total flux @ element boundary EISE

Calc_gFlux $=0.0$

ENDIF

ENDIF

C

END

C This function calculates the specific heat

real FUNCTION Calc_Specific_Heat(Soil,WatCon,icecon)

C I Ensure that all variables have been correctly defined INCLUDE 'DECLARE.FI'

C

real Fn_Point
integer Soil

real WatCon,icecon

I (dec) The water content

C

integer $i$

real $t 1, t 2, t 3, t 4$

C

t1 $=\exp (X S H W C(1$, Soil $))$

t2 = exp(XSHWC(POINTS4(Soil),Soil))

if( WatCon.lt.t1) then

t2 $=\exp (X S H(1$, Soil $))$

t3 $=\exp$ XSH $(2$, Soil))

t4 = exp(XSHWC(2,Soil))

Calc_Specific_Heat $=02-((t 3-t 2) /((t-1)))^{*}(t 1-$ WatCon $)$

elseif( WatCon.gt.t2) then

$\mathrm{i}=$ POINTS4(Soil) - 1

$\mathfrak{t} 1=\exp ($ XSH(POINTS4(Soil),Soil))

t $3=\exp (X S H(i$, Soil))

t4 = exp(XSHWC(i,Soil))

Calc_Specific_Heat $=t 1+((t 1-t 3) /(t 2-t 4)) *($ WatCon-t2) 


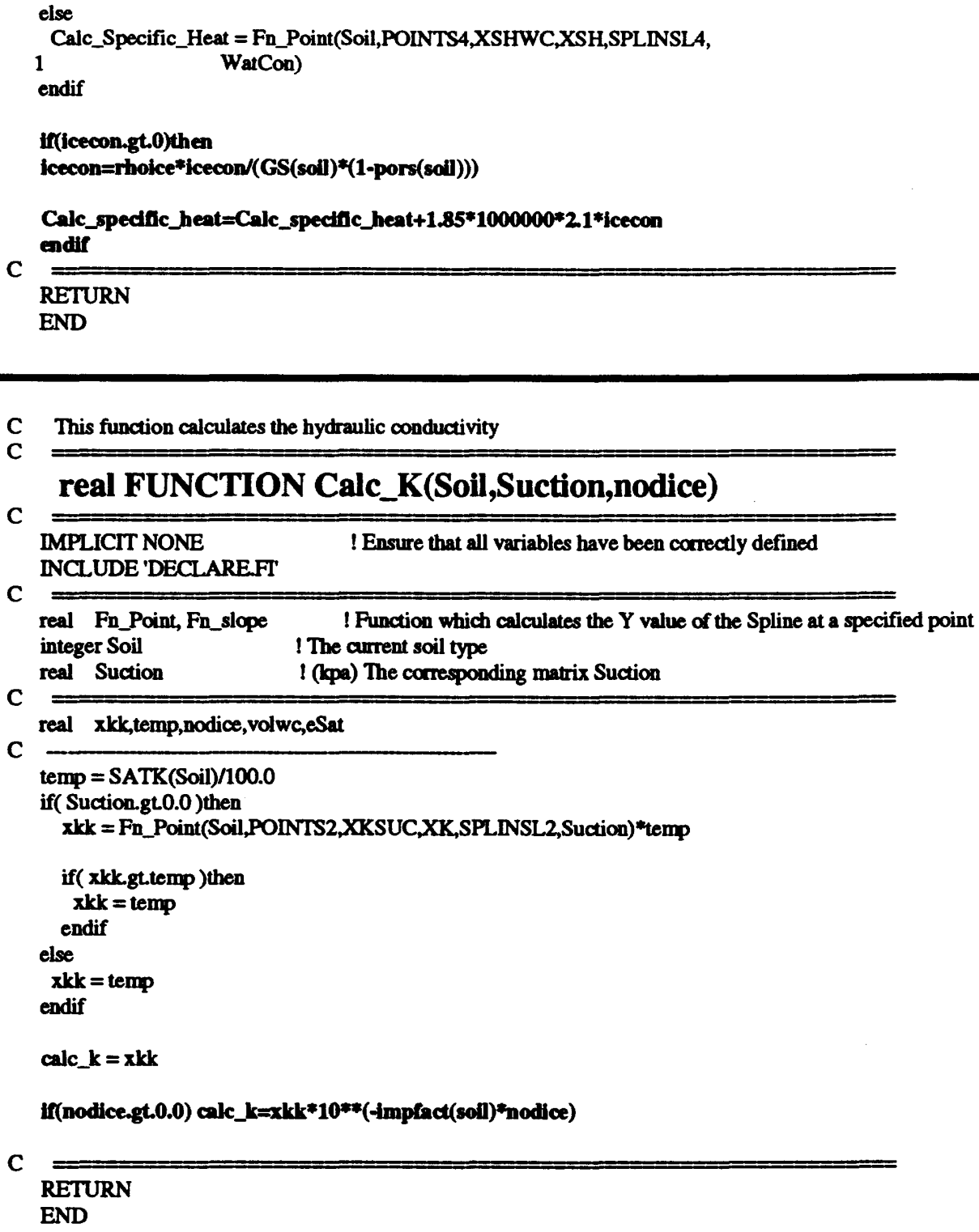

C This function calculates the hydraulic conductivity

C = = real FUNCTION Calc_K(Soil,Suction,nodice)

C ! Ensure that all variables have been correctly defined INCLUDE 'DECL.ARE.FI'

$C=$ real Fn_Point, Fn_slope I Function which calculates the $Y$ value of the Spline at a specified point integer Soil The current soil type real Suction $\quad$ ( (1pa) The corresponding matrix Suction

C real xkk,temp,nodice, volwc,esat

C

temp $=$ SATK(Soil) $/ 100.0$

if( Suction.gt 0.0 ) then

xkk = Fn_Point(Soil,POINTS2,XKSUC,XK,SPLINSL2,Suction)"temp

if ( xkk.gt.temp)then

xkk = temp

endif

else

xkk $=$ temp

endif

calc_k $=\mathbf{x k k}$

if(nodice.gt.0.0) calc_k=xkk*10**(-implact(soll)*nodice)

$\mathrm{C}=$

RETURN

END

\section{DOUBLE PRECISION FUNCTION Lump(R,C,m1,m2,lsys)}

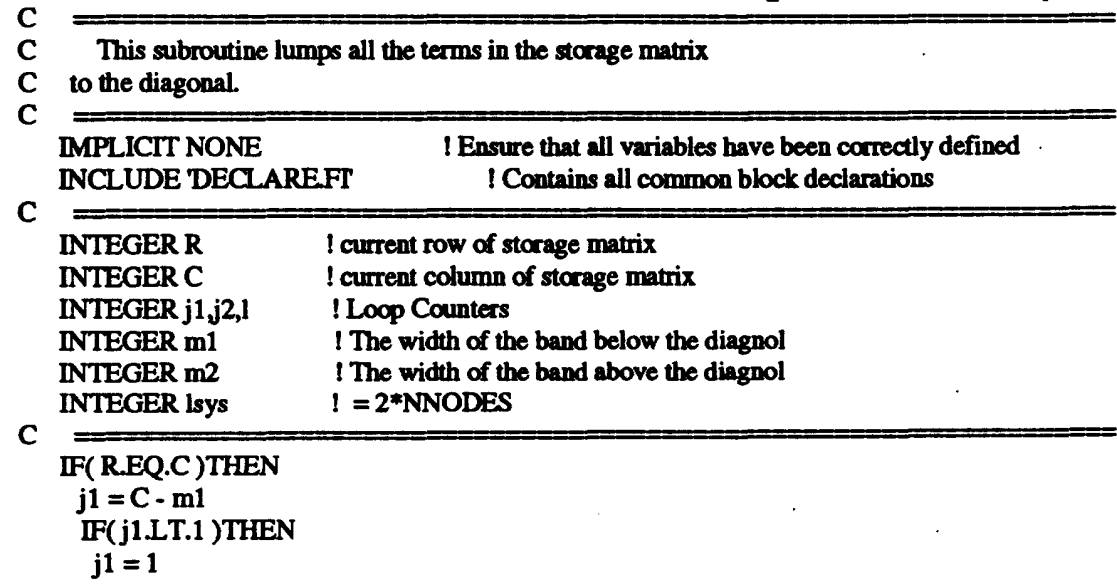


ENDIF

$\mathrm{j} 2=\mathrm{C}+\mathrm{m} 2$

IF(j2.GT.lsys )THEN

j2 $=$ lsys

ENDIF

Lump $=0.0 \mathrm{D} 0$

$\mathrm{DO} 1=\mathrm{j} 1 \mathrm{j} 2$

Lump $=$ Lump + SYSMAS(R,I)

ENDDO

ELSE

Lump $=0.0 \mathrm{D} 0$

ENDIF

C RETURN

RETU

C This function calculates a sin distribution for net radiation

C TA FUNCTION Calc NETRAD(Dayleng)!(MM/DAY)

c REAL FUNCTION Calc_NETRAD(Dayleng).(MM/DAY)
IMPLICIT NONE
! Ensure that all variables have been correctly defined

INCLUDE DECLARE.FT

! Contains all common block declarations

C REAL Dayleng ! ( HRS ) Number of hours in a day

REAL ea ! (mm/day) fu*ea*(B-A)

REAL sunrise ! ( HRS ) Hour of the day in which the sun rises (ie 12:00am =0)

REAL sunset ! ( HRS) Hour of the day in which the sun sets

REAL $q \max i(\mathrm{~W} / \mathrm{m} 2)$ Max net radiation

REAL timehrs ! (HRS ) current time

REAL gamma ! (kPa/C) Phsychrometer Constant

C IF(DFLUX.OR.STEADYSTATE)THEN ! Calculate an equivalent NetRad

Calc_NETRAD $=0.0$

ELSE

sunrise $=12.0-($ Dayleng/2.0)

sunset $=12.0+($ Dayleng/2.0)

timehrs $=$ ttime $/ 3600.0$

qmax $=$ PI * SOLAR(2) $/(2$ * (Dayleng * 3600)) *1.0E+6

$1 * 0.0353 ! \mathrm{W} / \mathrm{m} 2 \rightarrow \mathrm{mm} /$ day

IF((timehrs.GT.sunrise).AND.(timehrs.LT.sunset))THEN

1

Calc_NETRAD $=q \max * \operatorname{SIN}(P I *$ (timehrs - sunrise)

ELSE

/(sunset - sunrise))

Calc_NETRAD $=0.0$

ENDIF

ENDIF

C RETURN

END

C This source file provides the functions for the Cover Factors

$\mathrm{C}=$

REAL FUNCTION Calc_PlantLimitFactor(Suction)

C IMPLICIT NONE Insure that all variables have been correctly defined INCLUDE 'DECLARE.FI I INCLUDES ALL COMMON BLOCK DECLARATIONS

C REAL Suction ! Nodal Suction at Root Centroid

C

IF(Suction.LT.LimitingPt) THEN

Calc_PlantLimitFactor $=1.0$

ELSE IF(Suction.GT.WiltingPt) THEN

Calc_PlantLimitFactor $=0.0$

ELSE

C linear relationship 


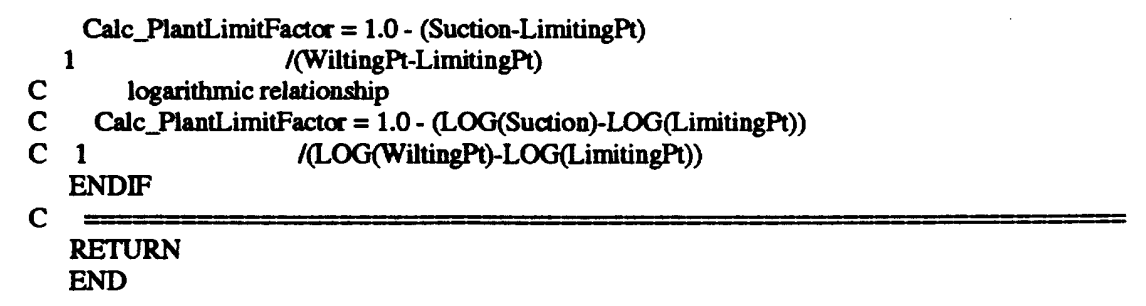

C This function calculates the potential root uptake for each node

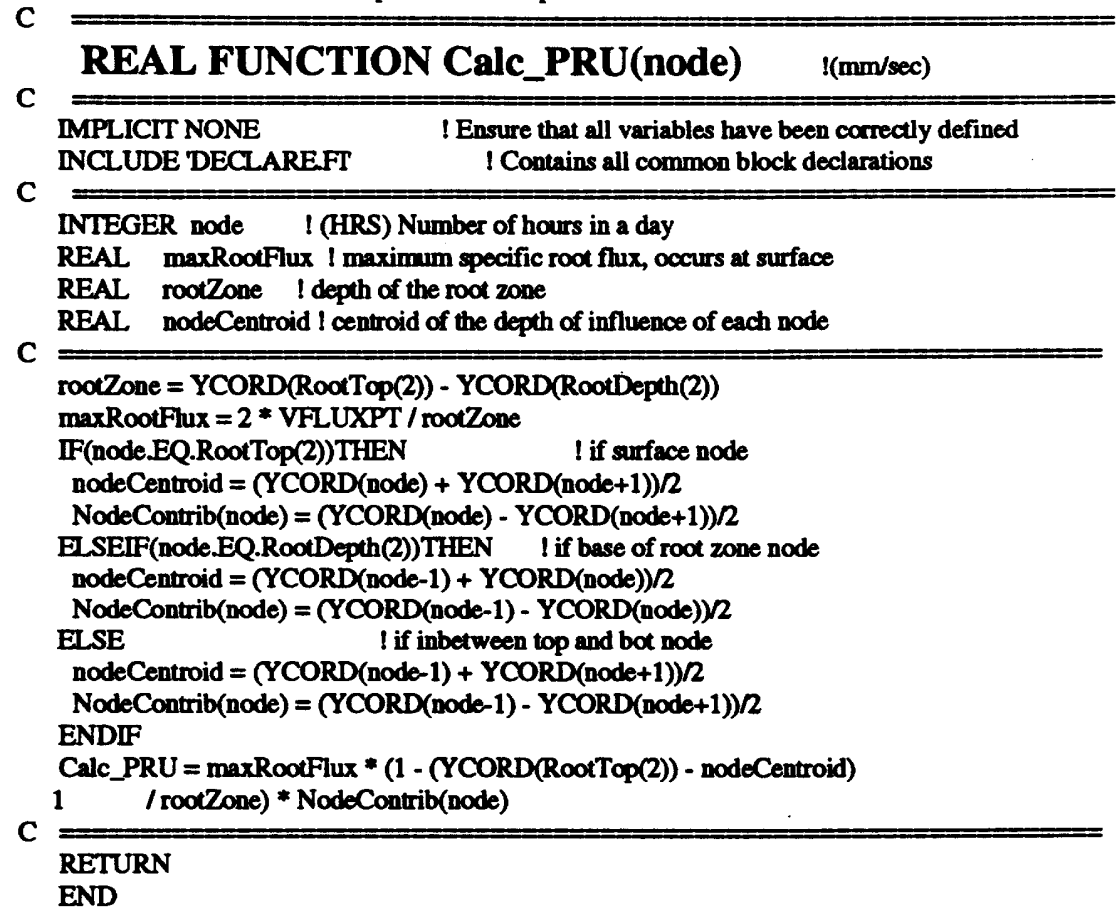

C This function calculates $\mathrm{Rh}$ given suction (kPa) and temperature (K)
(1) REAL FUNCTION Calc_RH(Suction,Temperature)
IMPLICIT NONE IEnsure that all variables have been correctly defined
$C$ COA
REAL Suction ! (kPa) Suction at Node or Guass Point
REAL Temperature! (K) Temperature of Node or Guass Point
$\mathrm{C}=$
IF(Suction.GE.0.0E0) THEN
Calc_RH $=\operatorname{EXP}((-2.1674 \mathrm{E}-03 *$ Suction $) / T e m p e r a t u r e)$
EISE
Calc_RH $=1.0 \mathrm{EO} 0$
ENDIF
C RETURN
END

C This function calculates change in volumetric water content per
C change in matric suction.

$\mathrm{C}=$

real FUNCTION Calc_RM2W(Soil,Suc) 
C

\begin{tabular}{|c|c|}
\hline $\begin{array}{l}\text { MMPLICIT NONE } \\
\text { INCLUDE 'DECLARE.FT' }\end{array}$ & ! Ensure that all variables have been correctly defined \\
\hline $\begin{array}{l}\text { integer Soil } \\
\text { real Fn_Slope } \\
\text { real Suc }\end{array}$ & $\begin{array}{l}\text { IThe current soil type } \\
\text { ! Function which calculates the slope of the Spline at a specified poin } \\
\text { ! (kpa) The matrix Suction @ } t\end{array}$ \\
\hline
\end{tabular}

C if(Suc.GT.XSUC1(Soil))then

Calc_RM2W =-Fn_Slope(Soil,POINTS1,XSUC,XVOLWC,SPLINSL1,Suc) else

Calc_RM2W = RM2WA(Soil)

endif

C RETURN

END

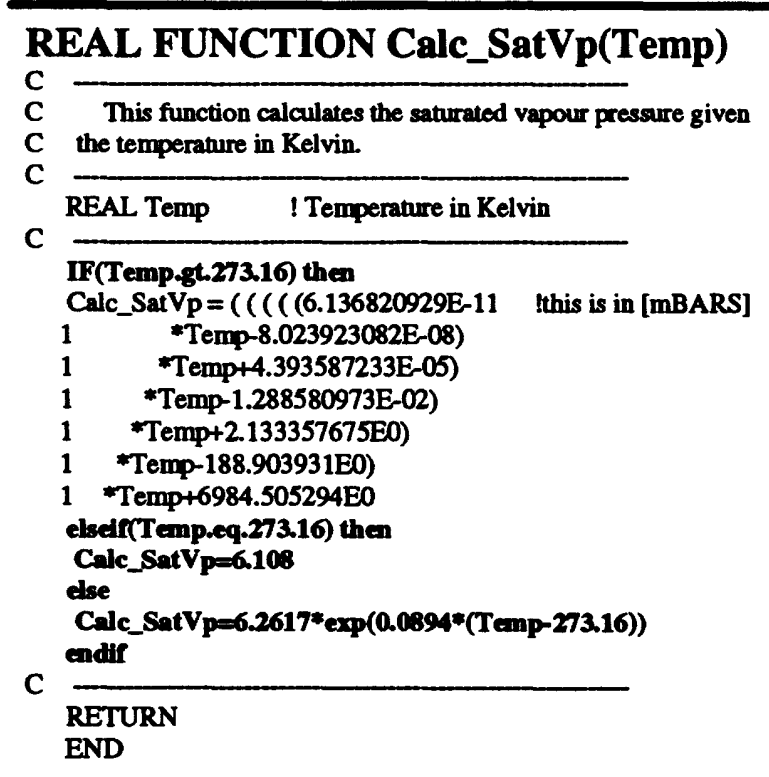

C

c Function to determine lapsed time from BASE

c A.E. Krause

c

C

\section{REAL FUNCTION SECNDS(BASE)}

C

IMPLICIT NONE

REAL BASE,TIME,LASTTIME

SAVE LASTTIME ! Must be saved to compare with next call

INTEGER IHR,IMIN,ISEC,I100TH

CALL GETTIM(IHR,IMIN,ISEC,I10OTH)

TIME $=\left(100.0 *\left(60.0 *\left(\mathrm{IMIN}+60.0^{*}\right.\right.\right.$ IHR $)+$ ISEC $\left.)+1100 \mathrm{TH}\right) / 100.0$

SECNDS=TIME-BASE

IF(BASE.EQ.0.0)THEN

LASTIIME $=0.0 \quad$ ! Initialize Lastime

EISE

IF(SECNDS.LT.LASTTIME)THEN ! If a new day has started

BASE $=$ BASE -86400.0

SECNDS = TIME-BASE

ENDIF

LASTTIME $=$ SECNDS

ENDIF

C

RETURN 
END

C This function calculates the soil temperature to be applied to the

C top node.

C

\section{real FUNCTION Calc_SoilTemp(TheWind) ! Celcius}

IMPLICIT NONE ! Ensure that all variables have been correctly defined

INCLUDE DECLARE.FT ! Contains all common variable declarations

C C

C Calculation of surface temperature (Wilson, 1990)

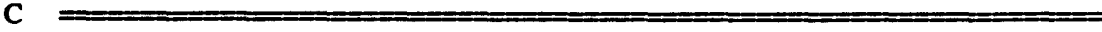
real TheWind $\quad !(\mathrm{km} / \mathrm{hr})$ specified Average Daily Wind Speed

C real air $\quad$ !(C) Current Air Temperature real fu $!(\mathrm{mm} / \mathrm{day} / \mathrm{kPa})$ function of Wind at $2 \mathrm{~m}$ real gamma I (kPa/C) Phsychrometer Constant real qstar_mod ! (dec.) qstar modifier based upon LAI

C

C CALCULATION OF CONSTANTS:

C Calculation of the Psychrometer Constant

c Linear Interpolation (Monteith, 1973) gamma $=6.41212 \mathrm{e}-05 *$ air $+0.064567273 ! \mathrm{kPa} C$

C Calculate the heat supplied by vapour in $\mathrm{mm} /$ day

c volwc $=$ Calc_VolWc(SOILTYPE(1),(SUCNOD(1)+SUCNOD(2))/2.) $!$ Volumetric Water Content

c grvwc $=$ volwc/(GS(SOILTYPE(1))*(1-PORS(SOILTYPE(1)))) ! Gravimetric Water Content

c lamda $=$ Calc_Thermal_Cond(SOILTYPE(1),grvwc) ! Thermal Conductivity

c qG = lamda*(TEM(1)-TEM(2))/(YCORD(1)-YCORD(2))*0.0353*100.

C Calculation of fu constant $\mathrm{fu}=2.6252 *(1+0.15 *$ TheWind $) \quad$ !(mm/day $) / \mathrm{kPa}$

C Calculation of the LAI - Qstar modifier IF(VEGETATION)THEN IF(LAI.LT.0.1)THEN qstar_mod $=1.0 *$ EXP(-MULCH) ELSEIF(LAI.GT.2.7)THEN gstar_mod $=0.0$ ELSE

qstar_mod $=\operatorname{EXP}\left(-0.4^{*}\right.$ LAI-MULCH)

ENDIF

ELSE

qstar_mod $=1.0$

ENDIF

C Calculation of surface temp Calc_soiltemp $=(1.0 /$ (gamma*fu $))^{*}$

1 (QSTAR*qstar_mod+PENMAN*86400.)+air ! (C) Note plus sign is for evap.

IF( Calc soiltemp.LT.TEMPAMIN(2))THEN

Calc_soiltemp $=$ TEMPAMIN(2) ENDIF

c if(calc_soiltemp.It.0.) Calc_soiltemp=air+(0.1*snowdepth(nday+1))

$\mathrm{C}=$

RETURN

END

C This function calculates the thermal conductivity

c real FUNCTION Calc_Thermal_Cond(Soil,WatCon,temp,nodeice)

MMPICIT NONE ! Ensure that all variables have been correctly defined 
INCLUDE 'DECLARE.FT'
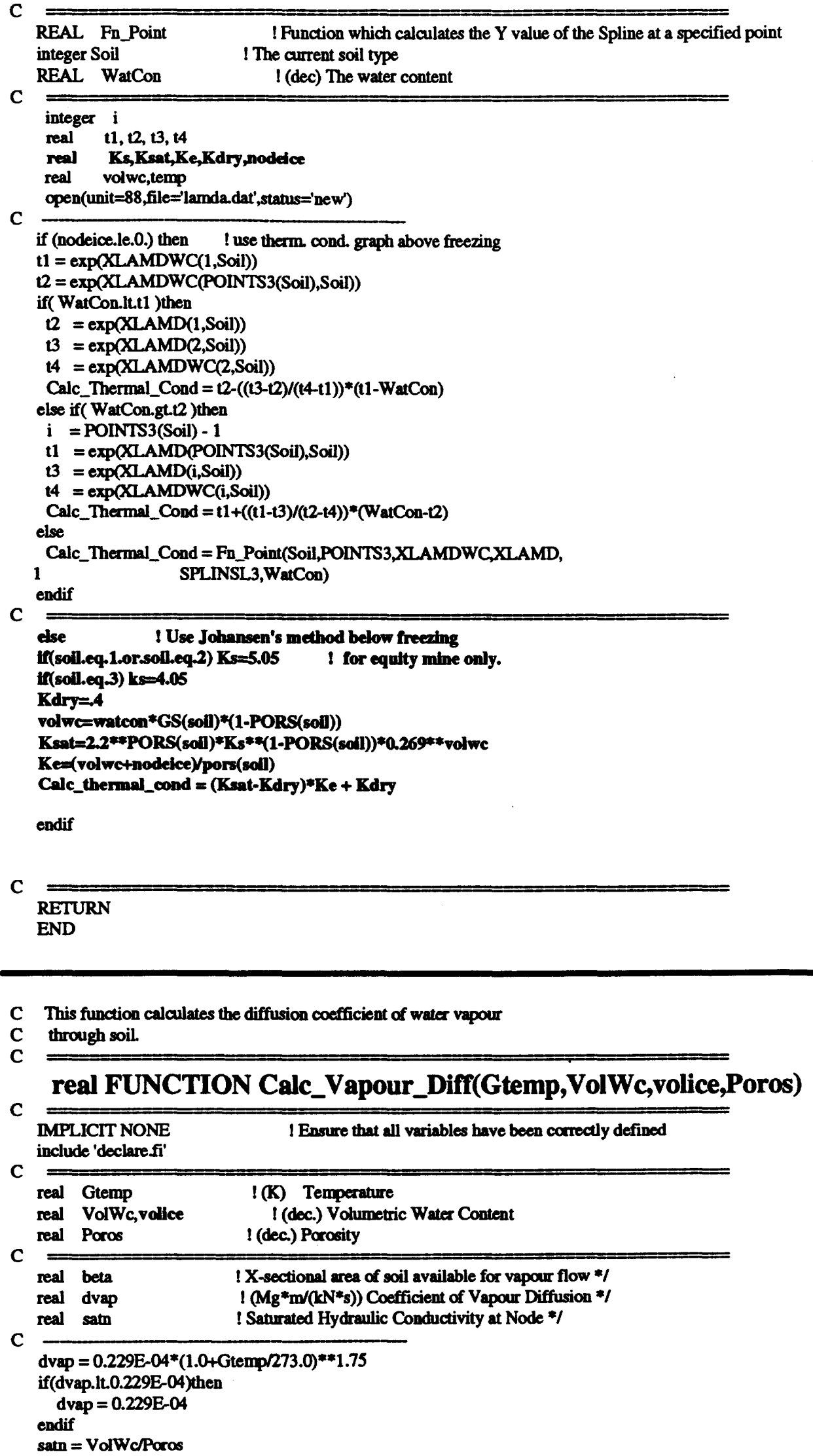
if(satn.gt.1.0)then

satn $=1.0$

endif

beta $=$ Poros-Volwc-volice

if(beta.lt.0.) then

beta $=0.0$

endif

Calc_Vapour_Diff $=2.1674 \mathrm{E}-03 *($ beta $* * 1.667) *$ dvap/Gtemp

RETURN

END

C This function calculates the vertical flux to be applied to the

C top node.

C REAL FUNCTION Calc_Vflux(RhSoil) ! (mm/sec)

C MMPICIT NONE I Ensure that all variables have been correctly defined INCLUDE 'DECLARE.FT

C

real ea $!(\mathrm{mm} /$ day) fu*ea*(B-A)

real fu $!(\mathrm{mm} /$ day $/ \mathrm{kPa})$ function of wind at $2 \mathrm{~m}$

REAL gamma ! (kPa/C) Phsychrometer Constant

REAL qstar_mod ! (dec) Modifies Rn by LAI function

REAL RhSoil ! (dec) Relative Humidity at Top Node

C IF(QSTAR.EQ.0.0)THEN

Calc_Vflux $=0.0$

ELSE

C Modified Penman Method

C $=$

C Calculation of the Psychrometer Constant

c Method 1: Linear Interpolation (Monteith, 1973)

gamma $=6.41212 \mathrm{e}-05 *($ TEMPAIR-273 $)+0.064567273$

c Method 2: Storr and Hartog gamma calculation (1975)

c gamma $=(6.50-4 *($ PVAIR1 $* 10)+6.35$ e-07* (PVAIR1 $* 10) *($ TEMPAIR-273)+

c $13.537 \mathrm{e}-07^{*}\left((\text { TEMPAIR-273)**2.0)*(RHAIR1*100.0) })^{*} 0.1\right.$

C Calculation of fu constant

C $\quad$ fu $=2.63^{*}(1.0+(0.537 / 3.6) *$ WIND(2)) ! original penman formulation

fu $=2.63^{*}(1.0+(0.864 / 3.6) *$ WIND(2)) tDocrenbos and Pruit (1977)

C Calculation of ea (mm/day)

if(thair 1.gt.0)then

ea = PVAIR 1*fu*(1/RHAIR1 - 1/RhSoil)

endif

C Calculating the LAI modifier

IF(VEGETATION)THEN

IF(LALLT.0.1)THEN

gstar_mod=1.0*EXP(-MULCH)

ELSEIF(LAI.GT.2.7)THEN

gstar_mod $=0.0$

ELSE

qstar_mod=EXP( $-0.4 *$ LAI-MULCH $)$

ENDIF

ElSE

qstar_mod $=1.0$

ENDIF

C Modified Penman Equation

Calc_Vflux $=(($ SLPOT1 * QSTAR * qstar_mod + gamma*ea) $)$

1 ((gamma*1/RhSoil) + SLPOT1) $) / 86400.0$

ENDIF

C

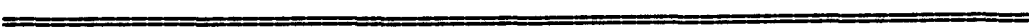


C This function calculates the vertical flux to be applied to the

C top node using the mass transfer method.

REAL FUNCTION Calc_Dflux(E) ! (mm/sec) IMPLICIT NONE I Ensure that all variables have been correctly defined INCLUDE 'DECLARE.FT

REAL E IVapour pressure of soil at the soil surface

C Modified Mass Transfer Method

C Calc_Dflux = -BCOEF(2)*(E-PVAIR1) RETURN END

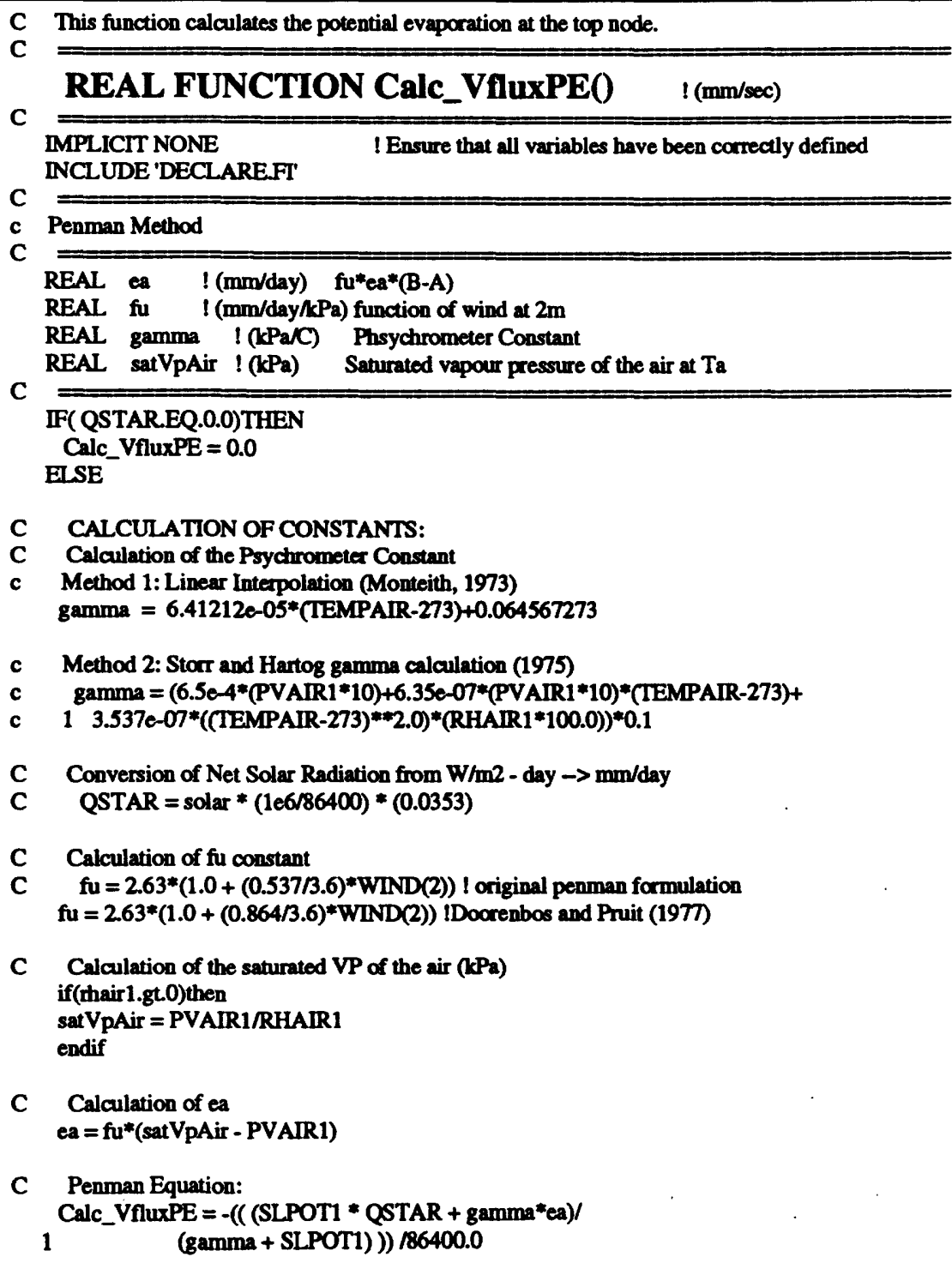

C This function calculates the potential evaporation at the top node.

C = REAL FUNCTION Calc_VfluxPE() !(mm/sec)

C IF( QSTAR.EQ.0.0)THEN

Calc_VfluxPE $=0.0$

ELSE

C CALCULATION OF CONSTANTS:

C Calculation of the Psychrometer Constant

c Method 1: Linear Interpolation (Monteith, 1973) gamma $=6.41212 e-05^{*}($ TEMPAIR-273 $)+0.064567273$

c Method 2: Storr and Hartog gamma calculation (1975)

c gamma $=\left(6.5 e-4 *(\right.$ PVAIR1 $* 10)+6.35 e-07 *\left(\right.$ PVAIR1 $\left.{ }^{*} 10\right) *($ TEMPAIR-273)+

c $13.537 \mathrm{e}-07 *(($ TEMPAIR-273)**2.0)*(RHAIR1*100.0) $) * 0.1$

C Conversion of Net Solar Radiation from W/m2 - day $\rightarrow$ mm/day

C QSTAR $=$ solar * $(1 \mathrm{e} 6 / 86400) *(0.0353)$

C Calculation of fu constant

C fu $=2.63^{*}\left(1.0+(0.537 / 3.6)^{*}\right.$ WIND(2) $)$ ! original penman formulation fu $=2.63 *(1.0+(0.864 / 3.6) *$ WIND(2)) !Doorenbos and Pruit (1977)

C Calculation of the saturated VP of the air (kPa) if(thair1.gt.0)then satVpAir = PVAIR1/RHAIR1 endif

C Calculation of ea $e a=f u^{*}$ (satVpAir - PVAIR1)

C Penman Equation: Calc_VfluxPE $=-(($ (SLPOT1 * QSTAR + gamma *ea $) /$ 1 $($ gamma + SLPOT1) )) $/ 86400.0$ 
ENDIF

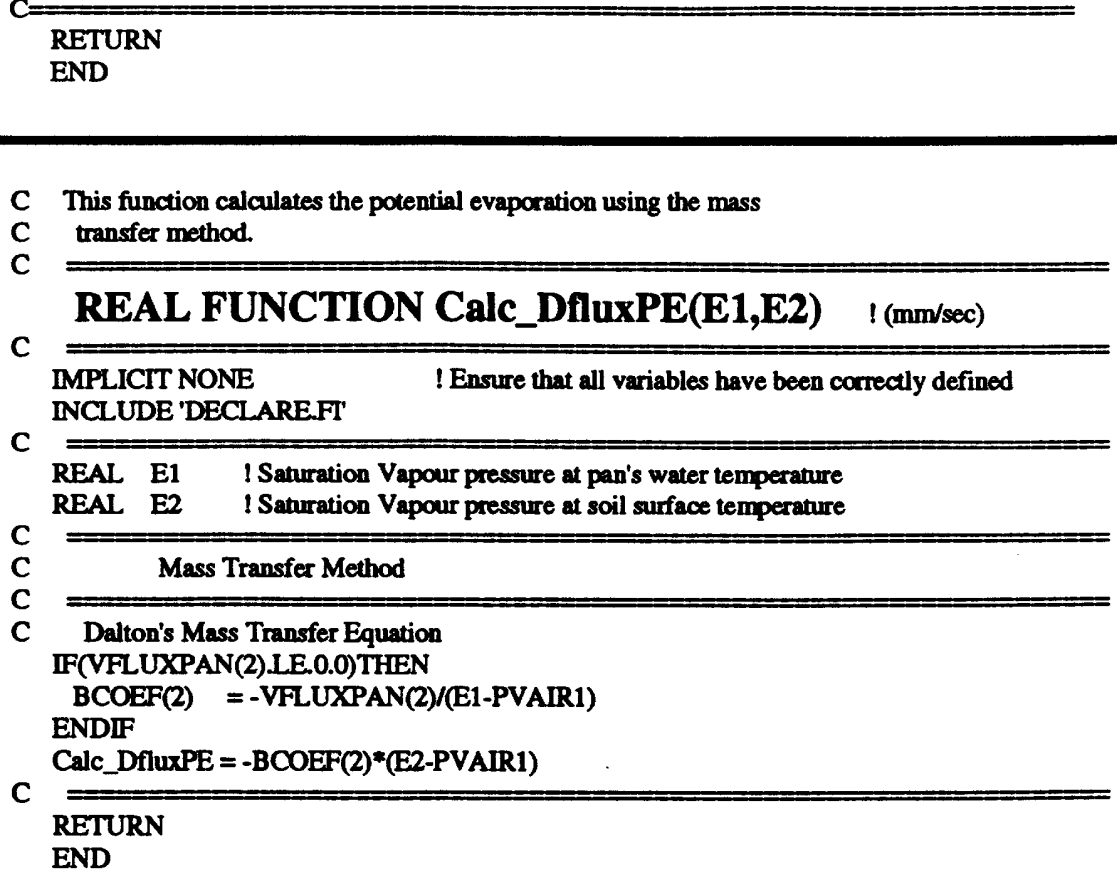

C This function calculates the POTENTIAL TRANSPIRATION

$\mathrm{C}=$

REAL FUNCTION Calc_VfluxPT0 !(mm/day)

IMPLICIT NONE I Ensure that all variables have been correctly defined INCLUDE 'DECLAREFI

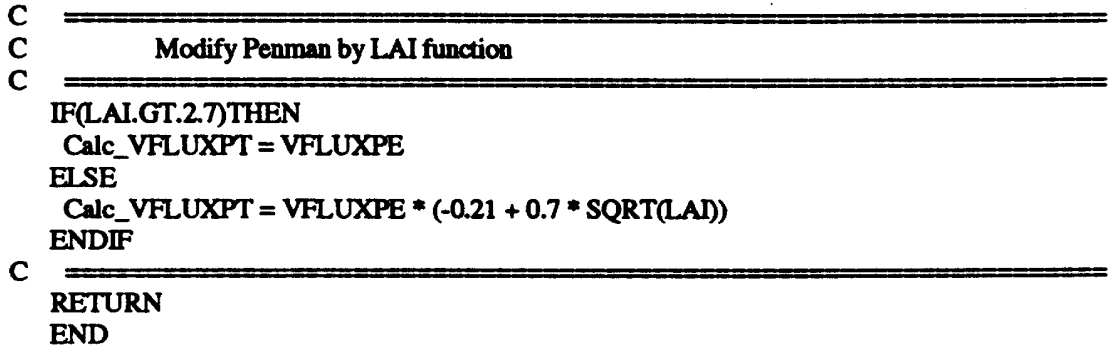

C This function calculates the volumetric water content

$\mathrm{C}=$

\section{REAL FUNCTION Calc_VolWc(soil,Suction)}
IMPLICIT NONE IEnsure that all variables have been correctly defined INCLUDE 'DECLARE.FI
$C$ CAL REAL_Fn_Point I Function which calculates the $Y$ value of the Spline at a specified point integer soil ! Temporary $\mathrm{x}$ variable REAL 12 ! Temporary variable
REAL Suction ! (kpa) The corresponding matrix Suction
$\mathrm{C}$

if(Suction.lt.XSUC1(soil))then

Calc_VolWc $=$ SUCT_INT(soil) - RM2WA(soil)*Suction else 
1

Calc_VolWc $=$ FN_POINT(soil,POINTS1,XSUC,XVOLWC,SPLINSL1,

endif Suction)

C

RETURN

END

C This function calculates the volumetric water content

\section{REAL FUNCTION WaterBalance()}

MPLICIT NONE

! Ensure that all variables have been correctly defined

INCLUDE FUNCTION.FT

INCIUDE 'DECLARE.FT

I All include files in this file

C Functions

C

REAL bottom I The flux at the bottom node

INTEGER $i, j$ l loop counters

INTEGER type

INTEGER node

REAL specified

! The current soil type

! The current node

REAL top

REAL $v 0$

The user specified flux

REAL v1

I The flux at the top node

I Initial vohume of water

double precision vt,ft

REAL volwc_i

REAL volwc $j$

! Final volume of water

SAVE vo,ft

IVolumetric water content at current node

! Volumetric water content at next node

v1 $=0.0$

type = SOILTYPE $(1)$

volwc $\mathbf{j}=$ Calc_VolWc(type,SUCNOD(1))

DO $\mathrm{i}=1$,(NNODES-1)

$\mathrm{j}=\mathrm{i}+1$

volwc $i=$ volwc $j$

type = SOILTYPE(j)

volwc $\mathbf{j}=$ Calc_VolWc(type,SUCNOD(j))

$\mathrm{v} 1=\mathrm{v} 1+(\mathrm{YCORD}(\mathrm{i})-\mathrm{YCORD}(\mathrm{j}))^{*}\left(\right.$ volwc_i $\left.+\mathrm{volwc}_{-}\right) / 2 \mathrm{lmm}$

$1 \quad+($ ycord $(i)$-ycord $(j)) *($ nodvolice $(i)+$ nodvolice $(j)) / 2$

1 thoice/thowat

ENDDO

C

IF( TTIME.EQ.DELTAT)THEN

$\mathrm{v} \mathbf{0}=\mathrm{v} \mathbf{1}$

$\mathrm{ft}=0.0 \mathrm{DO}$

ENDIF

top = VFLUX(1)

bottom $=$ VFLUX(NNODES)

DO $\mathrm{i}=1, \mathrm{NNBC}$

node $=$ NODEN $(1,2) \quad$ ! Node to apply boundary condition to

specified $=Q W(i, 2)$

IF( node.EQ.NNODES )THEN

IF( specified.NE.1.0E20)THEN ! Flag indicating atmospheric forcing

bottom $=$ specified $* 1000.0$

ENDIF

ENDIF

ENDDO

$\mathrm{vt}=\mathrm{vl}-\mathrm{vo}$

f $=f t+($ top-bottom + VFLUXAT $) * D E L T A T$

IF( NDAY.GT.0)THEN

WaterBalance $=\mathbf{v t}-\mathrm{ft}$

ELSE

WaterBalance $=0.0$

ENDIF

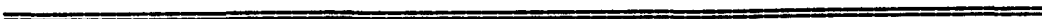




\section{APPENDIX F SUPPORT FILES REQUIRED BY PROGRAM}




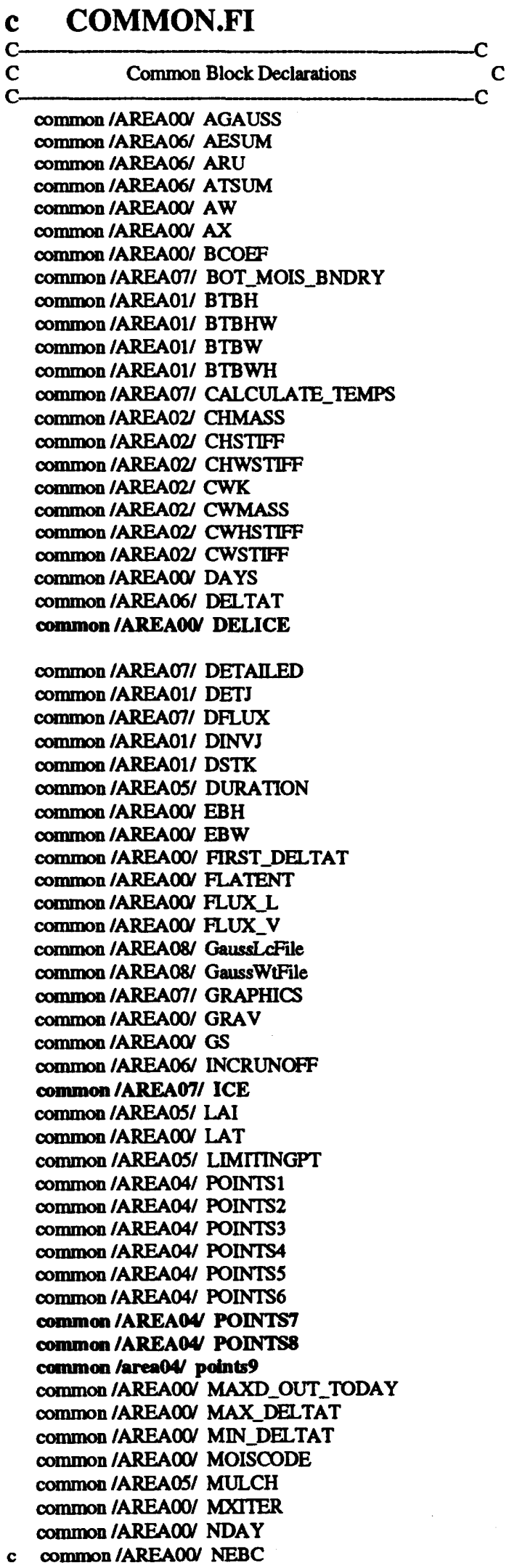


common /AREA00/ NELEM common /AREAOO/ NELCON

c common /AREA00/ NNBC common IAREAOO/ NNODES common /AREAOO/ NODEB common /AREA05/ NODECONTRIB common /AREAOO/ NODEN common/AREACO NODVOLICE

common /AREAOO/ oldnodvolice common/AREA00/ NSTART common /AREA08/ OUTPUT common /AREA06/ PENMAN common /AREA06/ PESUM common /AREA06/ PHIA common /AREA06/ PLANTSUM common IAREAOO/ PNODES common /AREAOOV PORS common /AREAOO/ PRESNOD common /AREAOO/ PRETNOD common /AREA05/ PRINTTIME common /AREA06/ PRU common /AREA05/ PTSUM common IAREAOO/ PUSNORM common /AREA00/ PUTNORM common /AREAOO/ PVAIR1 common IAREAOO QSTAR common IAREAOO/ QW common /AREA05/ RAIN common /AREAOO/ RH_LAG common IAREA0O/ RH_MAX common /AREAOOV RH_MIN common /AREA0O RHAIR1 common /AREAOON RHOWAT common /AREACO/ RHOICE common /AREA00/ RLATENT common /AREA00/ RM2WA common /AREA05/ ROOTDEPTH common/AREA05/ ROOTTOP common /AREAO6/ RUNOFF common /AREA0O SATK common /AREA00/ IMPFACT common /AREA06/ SFLUX common /AREA06/ SFLUXARU common /AREA06/ SFLUXI common /AREA06/ SFLUXPRU common /AREA06/ SFLUXV common /AREA07/ SHUTDOWN common /AREA00/ SLPOT1 common /AREA00/ nexttoptemp common /AREA0O/ SOLAR common /AREAOO/ SOLTYPE common /AREA04/ SPLINSL1 common /AREA04/ SPLINSL2 common /AREA04/ SPLINSL3 common /AREA04/ SPLINSL4 common /AREA05/ SPLINSL5 common /AREA06/ SPLINSL6 common /AREA06/ SPLINSL7 common /AREAO6/ SPLINSLS common /area 06/ splinst9 common /AREA07/ STEADYSTATE common /AREAOO/ STSH common /AREA00/ STSW common /AREAOO/ SUC_DAMP common /AREAOO/ SUCNOD common IAREA00 SUCT_INT common /AREA03/ SYSF common /AREA03/ SYSMAS common /AREA 03/ SYSTIF. common/AREAOO/ TEM_DAMP 
common /AREAOO/ TEM

common /AREA00/ TEMPAIR

common /AREAOO/ TEMPAMAX

common /AREA00/ TEMPAMIN

common /AREA0\%/ TEMPERATURE_LAG

common /AREACO/ TIMEO

common /AREAOO/ TOLS

common /AREA00/ TOLT

common /AREA07/ TOP_MOIS_BNDRY

common /AREA07/ TRANSIENT

common /AREA06/ TTIME

common /AREA07/ VEGETATION

common /AREA06/VFLUX

common /AREA06/ VFLUXAE

common /AREA06/ VFLUXAT

common /AREA06/VFLUXPAN

common /AREA06/VFLUXPE

common /AREA06/ VFLUXPT

common /AREA05/ WILTINGPT

common IAREAOO WIND

common /AREAOO/WTWC

common /AREA04/ XK

common /AREA04/ XKSUC

common /AREA04/ XIAI

common /AREA05/ XIAIDAY

common /AREA04/ XILAMD

common /AREA04/ XLAMDWC

common /AREA04/ XMULCH

common IAREA04/ XMULCHDAY

common /AREA04/ XSH

common /AREA04/ XSHWC

common /AREA04/ XSUC

common IAREA04/ XSUC1

common /AREA04/ XVOLWC

common /AREAOO/ YCORD

common /AREAO4 XVOLUWC

common /AREAOA XTEM

common /AREAOS YVOLUWC

common /AREAOA YTEM

common hrea04 xeg

common /area 04 gvoluwe

$\begin{array}{lccll}\mathrm{C} & \text { Constant.fi } & \mathrm{C} & & \\ \mathrm{C} & \mathrm{C} & & \\ \mathrm{C} & \text { This is an include file for Vap1.for. } & \mathrm{C} & \mathrm{C} \\ \mathrm{C} & \text { This file provides the constants for the soilcover program } & \mathrm{C} & \mathrm{C} \\ \mathrm{C} & & \end{array}$

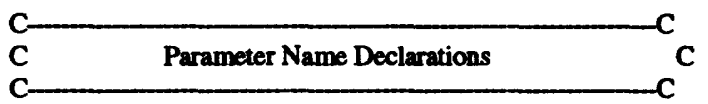

INTEGER MAX_DAYS ! Number of Simulation Days

INTEGER NEBC ! In general this is a variable, but for our purposes, it is constant

INTEGER NNBC ! In general this is a variable, but for our purposes, it is constant

c We will al ways have 2 , one at the top node $\&$ one at the bottom node

INIEGER MAX_EBC ! Number of Essential Boundary Conditions (Head type)

INTEGER MAX ELEM ! Number of Elements

INTEGER MAX_GAUSS ! Number of Gauss Points

INTEGER MAX_NBC I Number of Natural Boundary Conditions (Flux type)

INTEGER MAX_NODES ! Number of Nodal Points

INTEGER MAX_NODES 2 ? Twice the Number of Nodal Points

INTEGER MAX_PNODES I Number of Nodes per Elements

INTEGER MAX_POINTS ! Number of Specified Points for a Spline

INTEGER MAX_TYPES ! Number of Soil Types

REAL PI ! PI

REAL TINY ! A very small number 


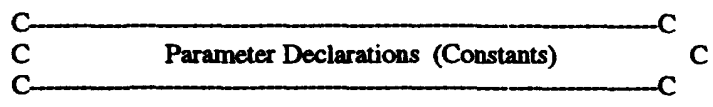

PARAMETER (MAX_DAYS = 3 ) ! Only 2 days are required, since 1 day is read in advance

PARAMETER (NEBC $=2$ ) ! This program is only currently able to use 2 EBC's

PARAMETER (NNBC $=2$ ) ! This program is only currently able to use 2 NBC's

PARAMETER (MAX_EBC $=2$ )

PARAMETER (MAX_ELEM $=100) !=($ MAXNODES -1$)($ PNODES -1$)$

PARAMETER (MAX_GAUSS $=7$ ) ! Must be greater than or equal to MAX_PNODES

PARAMETER (MAX_TYPES $=10$ )

PARAMETER (MAX_NBC $=3$ )

PARAMETER (MAX_NODES $=105$ )

PARAMETER (MAX_NODES $\times 2=210$ ) $!=$ MAX_NODES * 2

PARAMETER (MAX_PNODES $=3$ )

PARAMETER (MAX_POINTS $=40$ )

PARAMETER (PI = 3.14159265359)

PARAMETER (TINY $=1.0 \mathrm{E}-20$ )

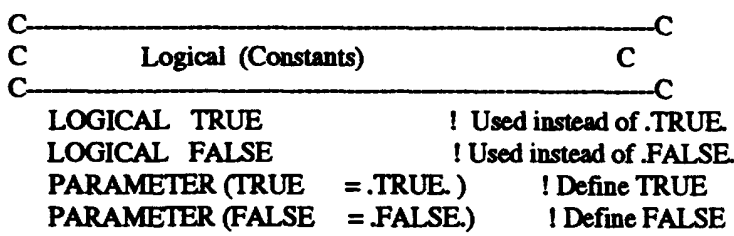

\begin{tabular}{ll} 
C & Graph Types (Constants) \\
C & C \\
\hline
\end{tabular}

integer linear,semi_log,logarithmic

parameter (linear $=1$ )

parameter (semi_log $=2$ )

parameter (logarithmic $=3$ )

\section{This is Declare.fi}

C Parameter Name Declarations CCC C C C

INCLUDE 'CONSTANT.FT ! Has all the constant declarations

C Variable Declarations for the Common Blocks C C

INTEGER AGAUSS ! Number of Gauss Points

DOUBLE PRECISION AEsum I Counter for the actual evaporation

DOUBLE PRECISION ARU (MAX_NODES) ! $!(\mathrm{mm} / \mathrm{sec})$ ACT ROOT UPTAKE FOR EACH TIME STEP

DOUBLE PRECISION ATsum I ! $m$ m/day) ACT TRANSP FOR EACH TIME STEP

REAL AW (MAX_GAUSS) I Temporary Storage for Gauss Points Wgts

REAL AX (MAX_GAUSS) ITemporary Storage for Gauss Point Locations

REAL BCOEF (MAX_DAYS) ! B Coefficient for the mass transfer method

REAL BTBH (MAX_PNODES,MAX_PNODES) ! Element Stiffness Matrix associated with heat flow

flow

REAL BTBHW (MAX_PNODES,MAX_PNODES) !Element Stiffness Matrix associated with heat coupled to moisture

REAL BTBW (MAX_PNODES,MAX_PNODES) ! Element Stiffness Matrix associated with moisture flow

REAL BTBWH (MAX_PNODES,MAX_PNODES) !Element Stiffness Matrix associated with moisture coupled to heat

flow

REAL CHMASS (MAX_GAUSS)

REAL CHSTIFF (MAX_GAUSS)

REAL CHWSTIFF(MAX_GAUSS)

REAL CWK (MAX_GAUSS)

REAL CWMASS (MAX_GAUSS)

REAL CWSTIFF (MAX_GAUSS)
IElement Property Coefficients

IElement Property Coefficients

! Element Property Coefficients

! Element Property Coefficients @ t $+d t$

I Element Property Coefficients

!Element Property Coefficients 
REAL CWHSTIFF(MAX_GAUSS) ! Element Property Coefficients

INTEGER DAYS

! Number of Days to Run Simulation

DOUBLE PRECISION DELTAT !Current time step in seconds

REAL EBH (MAX_EBC,MAX_DAYS) ! Essential Boundary Condition for temperature

REAL EBW (MAX_EBC,MAX_DAYS) ! Essential Boundary Condition for suction

REAL DELICE (max_nodes)

! Nodal change in ice content over time step

REAL DETJ (MAX_GAUSS) ! Determinant of Jacobian

REAL DINVJ (MAX_GAUSS) ! Inverse of Jacobian

REAL DSTK (MAX_PNODES) IElement load vector related to gravity

REAL DURATION(MAX_DAYS) IFlag which implements precip. ramp function

REAL FIRST_DELTAT I Initial Daily Time Step in seconds

DOUBLE PRECISION FLUX_L(MAX_NODES) ! $\quad \mathrm{mm} / \mathrm{s})$ Liquid flux at the element boundary

DOUBLE PRECISION FLUX_V(MAX_NODES) ! $(\mathrm{mm} / \mathrm{s})$ Vapour flux at the element boundary

CHARACTER*10 GaussLCFile ! File of Gauss Pt Locations

CHARACTER*10 GaussWtFile IFile of Gauss Pt Weights

REAL GRAV $!\left(\mathrm{m} / \mathrm{s}^{\wedge}\right)$ Accelaration due to gravity

REAL GS (MAX_TYPES) I Specific Gravity of Each Soil Layer

DOUBLE PRECISION INCRUNOFF ! Instantaneous Runoff rate (mm/s)

REAL LAI !Leaf Area Index for each day

REAL LAT IDegrees latitude of the site

REAL LimitingPt ! Limiting point on Plant Limiting Factor function

REAL MAXD_OUT_TODAY I Counter for TTMME that system didn't converge

REAL MAX_DELTAT

REAL MIN_DELTAT

INTEGER MOISCODE

REAL MULCH

INTEGER MXITER

INIEGER NDAY

c INTEGER NEBC

! Maximum Time Step Allowed in seconds

! Minimum Time Step Allowed in seconds

! Flag, 2 = specified initial nodal watercontents

! Daily mulch LAI value

! Maximum Number of Iterations

! Current Simulation Day

INTEGER NELCON (MAX_PNODES, MAX_ELEM) ! Element Connectivity Matrix

INTEGER NELEM

INIEGER NNODES

c INTEGER NNBC

INTEGER NSTART

INumber of elements

! Number of Nodes

I Number of Natural Boundary Conditions

! Number of days from January 1st

INTEGER NODEB (MAX_EBC,MAX_DAYS) ! Node to which EBC is to be applied

REAL NodeContrib (MAX_NODES) ! Contributing thickness of each node

INTEGER NODEN (MAX_NBC+1,MAX_DAYS) I Node to which NBC is to be applied

REAL NODVOLICE (MAX_NODES) I Ice storage at each node

REAL OLDNODVOLICE (MAX_nodes)

CHARACTER*11 OUTPUT

DOUBLE PRECISION PENMAN

DOUBLE PRECISION PESUm

REAL PHIA (MAX_NODES $\times 2$ )

DOUBLE PRECISION PLANTSUM

INTEGER PNODES

INTEGER POINTS1 (MAX TYPES)

INTEGER POINTS2 (MAX_TYPES)

! Output File Name

I (mm/day) Evaporation rate calculated by the modified penman method

t counter for the PE flux

I Suctions and Temps for Solver

1 Counter for PLANT ROOT FLUX

I Number of Nodes per Element

I Number of Data Pts. in VolWc vs Suction

I Number of Data Pts. in Suction vs Permeability

I Number of Data Pts. in ThermCond vs Wc by Wgt

INTEGER POINTS4 (MAX_TYPES)

! Number of Data Pts. in SpecHeat vs Wc by Wgt

I Number of Data Pts. in GREEN LAI vs Day

I Number of Data PLs. in MULCH LAI vs Day

I Number of Data Pts. in grav. water vs temp

INTEGER POINTS6 (MAX_TYPES)

INTEGER POINTS7 (MAXX_TYPES)

INTEGER POINTSS (MAX_TYPES)

integer points9 (max_types)

REAL PORS (MAX_TYPES)

REAL PRESNOD (MAX_NODES)

REAL PRETNOD (MAX_NODES)

INTEGER PrintTime

I Number of Data Pts. in neg. temp vs grav water content

Flag which specifies noon or midnight output

DULLE PRECISION PRU (MAX_NODES ) I $(\mathrm{mm} / \mathrm{sec})$ POT ROOT UPTAKE FOR EACH TIME STEP

DOUBLE PRECISION PTsum

REAL PUSNORM

REAL PUTNORM

REAL PVAIR1

REAL QSTAR

REAL QW

I Porosity of Each Layer

INodal Suctions from Previous Iteration

! Nodal Temperatures from Prev Iteration

REAL RAIN

REAL RH_LAG

REAL RH_MAX (MAX_DAYS )

REAL RH_MIN (MAX_DAYS)

! (mm/day) COUNTER FOR THE DAILY PT FLUX

! Specified Maximum Allowable Change in Suction Normal

! Specified Maximum Allowable Change in Temp Normal

I (kPa) Partial Vapour Pressure

1 (mm/day) Net solar radiation

I Rainfall rate minus runoff (used in display sub.)

I (HRS) Difference in time between daily peak $\mathrm{t}$ and daily Qnet peak

! Maximum Daily Relative Humidity of the air

! Minimum Daily Relative Humidity of the air 
REAL RHAIR1

REAL RHOWAT

REAL RHOICE

REAL RLATENT

REAL FLATENT

REAL RM2WA (MAX_TYPES)

INTEGER RooBepth (MAX_DAYS)

INTEGER RootTop (MAX_DAYS)

DOUBLE PRECISION RUNOFF

REAL SATK (MAX_TYPES)

REAL IMPFACT (max_types)

DOUBLE PRECISION SFLUX (MAX_NODES) ! (mm/day) Counter for Flux at each element boundary

DOUBLE PRECISION SFLUXARU(MAX_NODES) !(mm/day/cm) counter for act $\mathrm{rt}$ upt for each node DOUBLE PRECISION SFLUXI (MAX_NODES) ! (mm/day) Counter for Flux at each element boundary DOUBLE PRECISION SFLUXPRU(MAX_NODES) $\quad !(\mathrm{mm} / \mathrm{day} / \mathrm{cm})$ counter for pot $\mathrm{rt}$ upt for each node DOUBLE PRECISION SFLUXV (MAX_NODES) ! (mm/day) Counter for Flux at each element boundary

REAL SLPOT1 I (kPa/C) Slope of Sat VP vs Temp Curve at surface.

REAL SOLAR (MAX_DAYS ) I (MJ/m^2/day) Net Solar Radiation

real nexttoptemp ! next days user defined temp at surface

INTEGER SOILTYPE(MAX_NODES ) ! The soil type corresponding to the node

REAL SPLINSL1(MAX_POINTS,MAX_TYPES) ! Calculated Spline Wgts.

REAL_SPLINSL2(MAX_POINTS,MAX_TYPES) !Calculated Spline Wgts.

REAL SPLINSL3(MAX_POINTS,MAX_TYPES) ICalculated Spline Wgts.

REAL SPLINSL4(MAX_POINTS,MAX_TYPES) !Calculated Spline Wgts.

REAL SPLINSL5MAX_PONTS,MAX_TYPES) ! Calculated Spline Wgts.

REAL SPLINSL6(MAX_POINTS,MAX_TYPES) !Calculated Spline Wgts.

REAL SPLINSL7(MAX_POINTS,MAX_TYPES) I Calculated Spline Wgts.

REAL SPLINSLS(MAX_POINTS,MAX_TYPES) ICalculated Spline Wgts.

real splinsiS(max_polnts,max_types)

REAL STSW (MAX_PNODES,MAX_PNODES) !Element Mass Moisture Storage Matrix

real stswh (max_pnodes, max_pnodes) ! mass storage related to temp. below freezing

REAL STSH (MAX_PNODES,MAX_PNODES) ! Element Mass Heat Storage Matrix

REAL SUC_DAMP

ISuction Dampening coefficients

REAL SUCNOD (MAX_NODES)

REAL SUCT_INT(MAX_TYPES)

REAL SYSF (MAX_NODESX2) $1(\mathrm{kPa})$ Nodal Suctions

I (kPa) The suction intercept of the Moist. Retent. Curve

OEX2,MAX NODES 2 ) ! The global mass storage matrix

REAL SYSTIF (MAX_NODES×2,MAX_NODESx2) ! The global Stiffness matrix

REAL TEM_DAMP I Temperature Dampening coefficient

REAL TEMPAIR

I(K) The current air temperature

REAL TEM (MAX_NODES)

REAL TEMPAMAX (MAX_DAYS)

REAL TEMPAMIN (MAX_DAYS)

REAL TEMPERATURE LAG

REAL TIMEO

REAL TOLS

REAL TOLT

DOUBLE PRECISION TTIME

DOUBLE PRECISION VFLUX DOUBLE PRECISION VFLUXAE

DOUBLE PRECISION VFLUXAT I (C) Nodal Temperatures

I(C) Max air temp

! (C) Min air temp

! (HRS) Difference in time between daily peak temp and daily Qnet peak

! (sec) Initial time at start of run.

I Time Step Tolerence for Nodal Suctions

I Time Step Tolerence for Nodal Temperatures

! Total elapsed time in seconds of current day

DOUBLE PRECISION VFL UXPAN(MAX_DAYS) $!(\mathrm{m} / \mathrm{s})$ pan evaporation

DOUBLE PRECISION VFLUXPE $\quad !(\mathrm{m} / \mathrm{s})$ potential evaporation

DOUBLE PRECISION VFLUXPT $\quad !(\mathrm{m} / \mathrm{s})$ potential transpiration

REAL WiltingPt

! Wilting Point on Plant Limiting Factor function

REAL WIND (MAX_DAYS ) I ( $\mathrm{cm} / \mathrm{mr})$ Average Daily Wind Speed

REAL WTWC (MAX NODES) IGravimetric Nodal Water Contents

REAL XK (MAX_POINTS,MAX_TYPES) I Permeability Data Points for Suction vs Permeability

REAL XKSUC (MAX_POINTS,MAX_TYPES) I Suction Data for Suction vs Permeability

REAL XILAI (MAX_POINTS,MAX_TYPES) ! GREEN Leaf Area Index for GREEN LAI vs Day

REAL XIAIDAY (MAX_POINTS,MAX_TYPES) ! Day imput for GREEN LAI vs Day

REAL XIAMD (MAX_POINTS,MAX_TYPES) ! Thermal Conductivity Data for TC vs WC

REAL XIAMDWC (MAX_POINTS,MAX_TYPES) ! WaterContent by Wgt Data for Therm Cond. vs WC

REAL XVOLUWC (MAX_POINTS,MAX__TYPES) IUnfromen Vol. Water Content for UWc vs. Temp.

REAL XTEM (MAX_POINTS,MAX_TYPES) IT Temp. data for UWc vs. Temp.

REAL YVOLUWC (MAX_POINTS,MAX_TYPES) I Unfreaen vol. water content for TEMP vs UWC curve

REAL YTEM (MAX_POINTS,MAX_TYPES) I Temp. data for TEMP vs UWc curve

REAL XMULCH (MAX_POINTS,MAX_TYPES) I MULCH LAIFOR MULCHLAI vs DAY CURVE

REAL XMULCHDAY (MAX_POINTS,MAX_TYPES) ! DAY INPUT FOR MULCH LAI vs DAY CURVE

REAL XSH (MAX_POINTS,MAX_TYPES) ISpec. Heat Data for Sp.Heat vs WC

REAL XSHWC (MAX_POINTS,MAX_TYPES) ! WC by Wgt. Data fo Sp.Heat vs WC 
REAL XSUC (MAX_POINTS,MAX_TYPES) ! Suction Data for Suction vs. Perm.

REAL XSUC1 (MAX_TYPES) ! Initial Suction Point in Suction vs. Perm.

REAL XVOLWC (MAX_POINTS,MAX_TYPES) ! Volumetric Water Content for Suct. vs WC

REAL YCORD (MAX_NODES) ! Nodal Coordinates

real $x g g$ (max_points,max_types)

real gvoluwc (max_points,max_types)

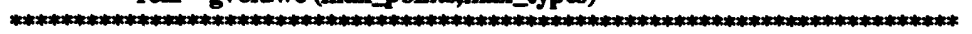

* Logical variables and definitions

*

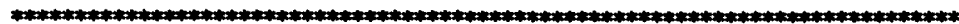

LOGICAL BOT_MOIS_BNDRY(MAX_DAYS) I Botom Boundry is specified as Volumetric Water Content

LOGICAL DETAILED

LOGICAL DFLUX

! Logical switch set when detailed output is required.

LOGICAL GRAPHICS !Logical switch to allow GRAPHICS information to be output.

LOGICAL ICE

LOGICAL SHUTDOWN

LOGICAL STEADYSTATE

I Flag indicating freeze/thaw is to be modelled

! Flag indicating runoff

I Flag indicating a steady state analysis is required

IFlag indicating to intrisically calc surface temperatures

LOGICAL TOP_MOIS_BNDRY(MAX_DAYS) ! Top Boundry is specified as Volumetric Water Content

LOGICAL TRANSIENT IFlag indicating a transient solution is required

LOGICAL VEGETATION IFlag indicating vegetation is to be modelled

$\mathrm{C}=$

INCLUDE 'CBLOCKS.FI' IContains all the common block declarations

C FUNCTION.FI

C Function Name Declarations

C $==$

real Calc_Airtemp ISin distribution for air temp

real Calc_AirRH I Sin distribution for relative humididty

real Calc_DayLeng ! Calculates the length of the day

real Calc_Dlux

real Calc_DfluxPE

real Calc_dicedTEM

real Calc_dicedsuc

real Calc_dicedt

real Colc_bellux

real Calc_guess_newtem

real Calc_K

real Calc_Netrad

real Calc_RH

real Calc_PlantLimitFactor

real Calc_PRU

real Calc_RM2W

real Calc_SatVp

real Calc_Soiltemp

real Calc_Specific_Heat

real Calc_Thermal_Cond

real Calc_Vapour_Diff

real Calc_Vfhux

real Calc_VfluxPE

real Calc_VFLUXPT

real Calc_VolWc

logical Convergence

real Fn_Point

real Fn_Slope

character inkey

real Secnds

double precision Lump

1 Calculates AE Using Mass Transfer Method

1 Calculates PE

I Calculates the change in vollice per dTEM

1 Calculates the change in loe per change in action for CWMASS

I Calculates the change in vol. per dt

I Calc joe flux $\mathrm{m} \mathrm{m} / \mathrm{s}$

ICalculates a guessed new node temp to use to get ice content

I Calculates the hydraulic conductivity

I Sin distribution foc net radiation

1 Calculates the relative humidity

I Calculates the plant limiting factor

1 Calculates the Pot Root Uptake for each node

1 Calculates RM2W

1 Calculates the saturated vapour pressure (mbar)

1 Calculates the surface temperature of the soil

1 Calculates the specific heat

1 Calculates the thermal conductivity

1 Calculates the vapour diffusion

1 Calculates AE Using The Modified Penman Method

! Calculates PE

! CALCULATES PT

I Calculates the volumetric water content

I Determines if system has converged

ICalculates the $Y$ value of the Spline at a specified point

1 Calculates the Slope of the Spline at a specified point

1 Calculates time in seconds for total run time.

I Returns the lumped storage terms on the current row.

C COMMONLY USED SPP PARAMETERS

c Define printer buffer size 


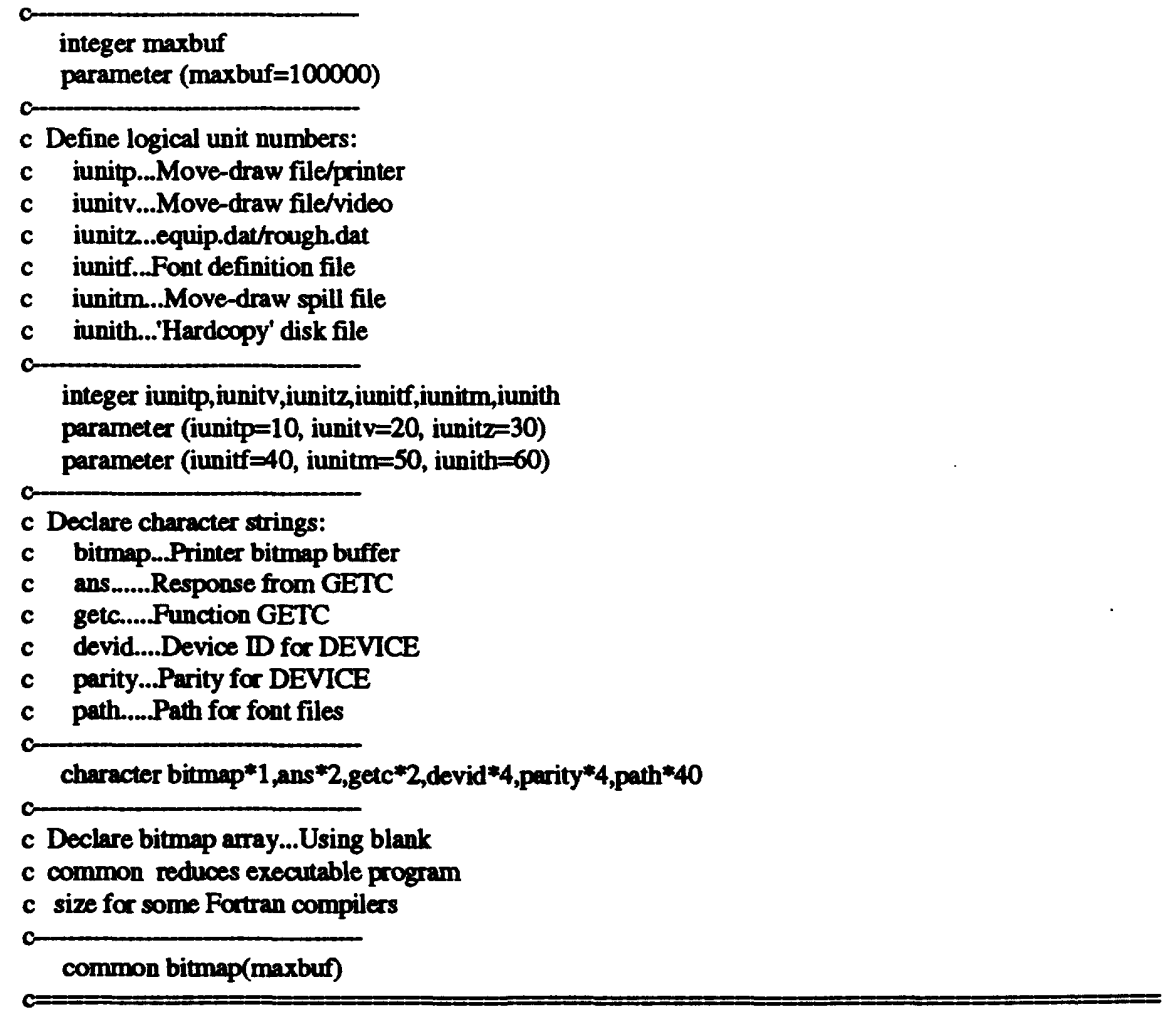

\section{File "Boards..dat"}

- 4-CGA Color.....320x200 '

- 5-CGA B\&W........3200200 .

- 6-CGA B\&W.......640×200'

' 13-EGA Color......320x200'

' 14-EGA Color.....640×200'

' 15-EGA Mono.......640×350 '

' 16-EGA Color.....640×350 '

- 17-MCGA \& VGA....640x480 '

' 18-VGA Coloc.....640×480

' 19-MCGA \& VGA....320×200'

' 37-Genca VGA.....640×480

' 39-Genca VGA......720x512 '

' 40-Hercules......720x348.

-41-Genoa/Orchid..800x600

' 45-Genoa EGA.....640×350 '

'46-Genca/Orchid..640x480'

' 47-Genoa VGA.....720x512 '

' 55-Genoa/Orchid.1024x768

' 72-AT\&T 6300.....640x400

' 88-Paradise VGA..800×600'

' 89-Paradise VGA..800 600 '

' 91-Genoa EGA.....640×350 '

- 92-Genca VGA.....640x480'

' 93-Genoe VGA.....720x512

'94-Paradise VGA..640x400'

' 95-Paradise VGA..640x480'

- 99-Tatung VGA....720x540

' 100 -Tatung VGA....800x600'

'115-Genoa VGA.....640×480

'121-Genoa VGA.....800x600'

'124-Genoa VGA.....512x512

'125-Genoa VGA.....512x512

'200-Everex VGA....640x480 
201-Everex VGA....752×410' '202-Everex VGA....800x600' 217-Everex EGA...1280×350 219-Everex EGA....640×350 ' '220-Everex VGA....640x400' 221-Everex VGA....512x480'

255-VESA SVGA**...800 600 256-VESA SVGA.....640x400 '257-VESA SVGA.....640×400' 258-VESA SVGA.....800 600 ' '259-VESA SVGA......800×600' 260-VESA SVGA.....1024x768 261-VESA SGVA....1024x768' '262-VESA SVGA...1280x1024' 263-VESA SVGA...1280x1024' '296-Video-7 EVGA..752X410' '297-Video-7 EVGA. 720x540' 298-Video-7 EVGA. $800 \times 600$ ' 299-Video-7 EVGA.1024x768 ' 300-Video-7 EVGA.1024x768' '301-Video-7 EVGA.1024x768' 302-Video-7 EVGA.640x400' '303-Video-7 EVGA. $640 \times 480$ ' '304-Video-7 EVGA. $720 \times 540$ ' '305-Video-7 EVGA. $800 \times 600$ ' '391-Trident EVGA. $800 \times 600$ ' '392-Trident EVGA.640x400' '393-Trident EVGA.640x480' 394-Trident EVGA. $800 \times 600$ ' '395-Trident EVGA.1024x768' '396-Trident EVGA.1024x768 ' '397-Trident EVGA.768x1024' '398-Trident EVGA.1024x768 '700-Wyse 700.....1280x800'

\title{
File "equip.dat"
}

\author{
mon $=16$ \\ ifore $=15$ \\ iback $=1$ \\ nprin $=8$ \\ mode $=5$ \\ isave $=-1$ \\ 'Ipt1' , 9600, 'none' , 1, 8, "
}

File "gauslc.dat"

$-0.57735$

0.57735

$-0.77459$

0

0.77459

$-0.86113$

$-0.33998$

0.33998

0.86113

$-0.90617$

$-0.53846$

0.53846

0.90617

$-0.93246$

$-0.6612$ 


\section{File "gauswt.dat"}

1.00

0.55555

$\mathbf{0 . 8 8 8 8 8}$

0.55555

0.34785

0.65214

0.65214

0.34785

0.23692

0.47862

0.56888

0.47862

0.23692

0.17132

0.36076

0.46791

0.46791

0.36076

0.17132

\section{Spline Smoothing Settings for soil type 1}

Suction vs Volumetric Water Content

Order \#Times

12

Suction vs Hydraulic Conductivity

Order \#Times

12

Gravimetric Water Content vs Thermal Conductivity

Order \#Times

12

Grav. W. C. vs Specific Heat

Order \#Times

12

Unfrozen $w / c$ vs. Temperature

Order \#Times

12

Spline Smoothing Settings for soil type 2

Suction vs Volumetric Water Content

Order \#Times

12

Suction vs Hydraulic Conductivity

Order \#Times

12

Gravimetric Water Content vs Thermal Conductivity

Order \#Times

12

Grav. W. C. vs Specific Heat

Order \#Times

12

Unfrozen w/c vs. Temperature

Order \#Times

12

Spline Smoothing Settings for soil type 3 
Suction vs Volumetric Water Content

Order \#Times

12

Suction vs Hydraulic Conductivity

Order \#Times

12

Gravimetric Water Content vs Thermal Conductivity

Order \#Times

12

Grav. W. C. vs Specific Heat

Order \#Times

12

Unfrozen w/c vs. Temperature

Order \#Times

12

Spline Smoothing Settings for soil type 4

Suction vs Volumetric Water Content

Order \#Times

12

Suction vs Hydraulic Conductivity

Order \#Times

12

Gravimetric Water Content vs Thermal Conductivity

Order \#Times

12

Grav. W. C. vs Specific Heat

Order \#Times

12

Unfirozen w/c vs. Temperature

Order \#Times

12

Spline Smoothing Settings for soil type 5

Suction vs Volumetric Water Content

Order \#Times

12

Suction vs Hydraulic Conductivity

Order \#Times

12

Gravimetric Water Content vs Thermal Conductivity Order \#Times

12

Grav. W. C. vs Specific Heat

Order \#Times

12

Unfrozen w/c vs. Temperature

Order \#Times

12

Spline Smoothing Settings for soil type 6

Suction vs Volumetric Water Content

Order \#Times

12

Suction vs Hydraulic Conductivity

Order \#Times

12

Gravimetric Water Content vs Thermal Conductivity

Order \#Times

12

Grav. W. C. vs Specific Heat

Order *Times

12

Unfrozen w/c vs. Temperature

Order \#Times

12

Spline Smoothing Settings for soil type 7 
Suction vs Volumetric Water Content Order \#Times

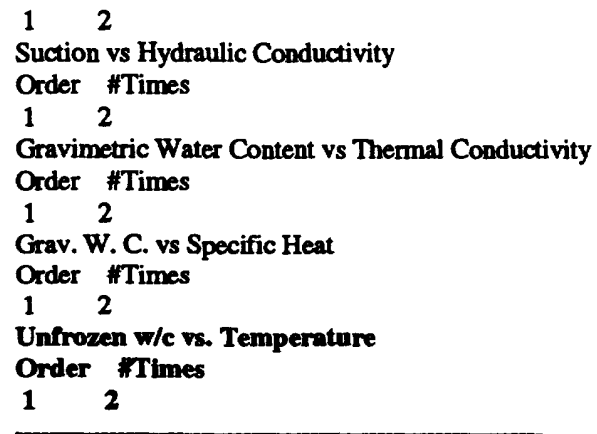

Spline Smoothing Settings for soil type 8

Suction vs Volumetric Water Content Order \#Times

12

Suction vs Hydraulic Conductivity

Order \#Times

12

Gravimetric Water Content vs Thermal Conductivity Order \#Times

12

Grav. W. C. vs Specific Heat

Order \#Times

12

Unfrozen w/c vs. Temperature

Order \#Times

12

Spline Smoothing Settings for soil type 9

Suction vs Volumetric Water Content

Order \#Times

12

Suction vs Hydraulic Conductivity

Order \#Times

12

Gravimetric Water Content vs Thermal Conductivity

Order \#Times

12

Grav. W. C. vs Specific Heat

Order \#Times

12

Unfrozen w/c vs. Temperature

Order \#Times

12

Spline Smoothing Settings for soil type 10

Suction vs Volumetric Water Content

Order \#Times

12

Suction vs Hydraulic Conductivity

Order \#Times

12

Gravimetric Water Content vs Thermal Conductivity

Order \#Times

12

Grav. W. C. vs Specific Heat

Order \#Times

12

Unfrozen $w / c$ vs. Temperature

Order \#Times

12 
GREEN LAI vs Day

Order \#Times

10

Spline Smoothing Settings for vegetation (MULCH)

MULCH LAI vs Day

Order \#Times

10

Raw Spline Data Output File

Suction vs Volumetric Water Content Soil \#Points First Point Last Point $1,0,5,500$

Suction vs Hydraulic Conductivity

Soil \#Points First Point Last Point

$1,0,1,400$

Gravimetric Water Content vs Thermal Conductivity

Soil \#Points First Point Last Point

$1,0,0.02,0.25$

Grav. W. C. vs Specific Heat

Soil \#Points First Point Last Point

$1,0,0.02,0.25$

Temperature vs unfrocen water content

Layer \#Points First Point Last Point

$1,0,0.02,0.25$

Unfrozen water content vs temperature

Layer \#Points First Point Last Point

$1,0,0.01,10$

dSUC/dTEM vs unfrocen water content

Layer \#Points First Point Last Point

$1,0,0.01,25$

GREEN LAI vs Day

Soil \#Points First Point Last Point

$1,0,0.01,5$

MULCH LAI vs Day

Soil \#Points First Point Last Point

$1,0,0.01,5$ 


\section{APPENDIX G SAMPLE INPUT FILES}




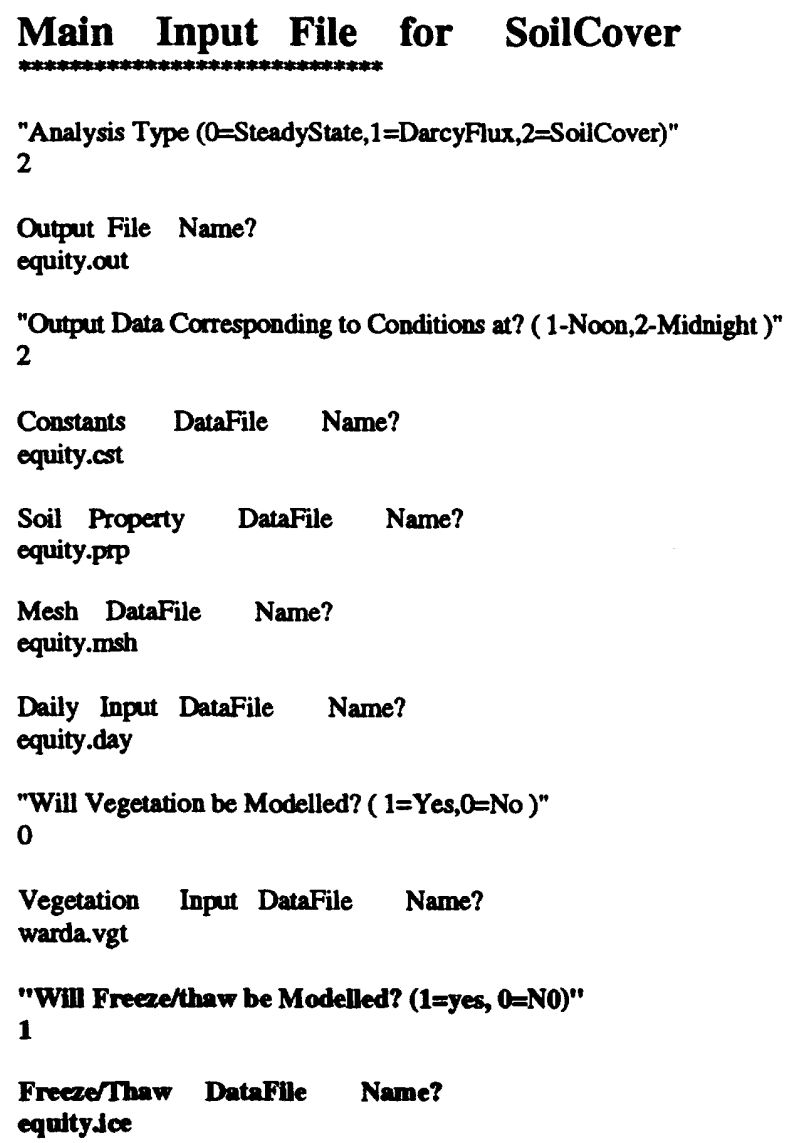




\begin{tabular}{|c|c|c|c|c|c|}
\hline Nod & & $\begin{array}{l}\text { il Elev } \\
\text { Type }\end{array}$ & $\begin{array}{r}\text { vation } \\
(\mathrm{cm})\end{array}$ & $\begin{array}{l}\text { MoistureCondition } \\
\text { (dec._or_kPa) (C) }\end{array}$ & Temperature \\
\hline 1 & 1 & 180 & 0.17 & 0 & \\
\hline 2 & 1 & 179 & 0.174 & 0 & \\
\hline 3 & 1 & 177 & 0.1756 & 60 & \\
\hline 4 & 1 & 175 & 0.1772 & 0 & \\
\hline 5 & 1 & 173 & 0.1788 & 0 & \\
\hline 6 & 1 & 171 & 0.1804 & 0 & \\
\hline 7 & 1 & 168 & 0.19 & 0 & \\
\hline 8 & 1 & 164 & 0.19 & 0 & \\
\hline 9 & 1 & 160 & 0.190 & 0.1 & \\
\hline 10 & 1 & 156 & 0.19 & 0.2 & \\
\hline 11 & 1 & 154 & 0.19 & 1.2 & \\
\hline 12 & 1 & 150.8 & 0.19 & 1.72 & \\
\hline 13 & 2 & 147.6 & 0.19 & 2.53 & \\
\hline 14 & 2 & 145.2 & 0.19 & 2.74 & \\
\hline 15 & 2 & 145 & 0.19 & 2.74 & \\
\hline 16 & 2 & 142 & 0.19 & 3.2 & \\
\hline 17 & 2 & 138 & 0.19 & 3.5 & \\
\hline 18 & 2 & 135 & 0.19 & 3.6 & \\
\hline 19 & 2 & 130 & 0.19 & 4.3 & \\
\hline 20 & 2 & 125 & 0.19 & 5.2 & \\
\hline 21 & 2 & 116 & 0.18 & 5.81 & \\
\hline 22 & 2 & 110 & 0.18 & 6.7 & \\
\hline 23 & 2 & 100 & 0.18 & 7.7 & \\
\hline 24 & 3 & 90 & 0.06 & 8.7 & \\
\hline 25 & 3 & 80 & 0.06 & 9.7 & \\
\hline 26 & 3 & 70 & 0.06 & 10.7 & \\
\hline 27 & 3 & 60 & 0.06 & 11.7 & \\
\hline 28 & 3 & 50 & 0.06 & 12.7 & \\
\hline 29 & 3 & 40 & 0.06 & 13.7 & \\
\hline 30 & 3 & 30 & 0.06 & 14.7 & \\
\hline 31 & 3 & 20 & 0.06 & 15.2 & \\
\hline 32 & 3 & 10 & 0.06 & 15.2 & \\
\hline 33 & 3 & 0 & 0.06 & 15.2 & \\
\hline
\end{tabular}

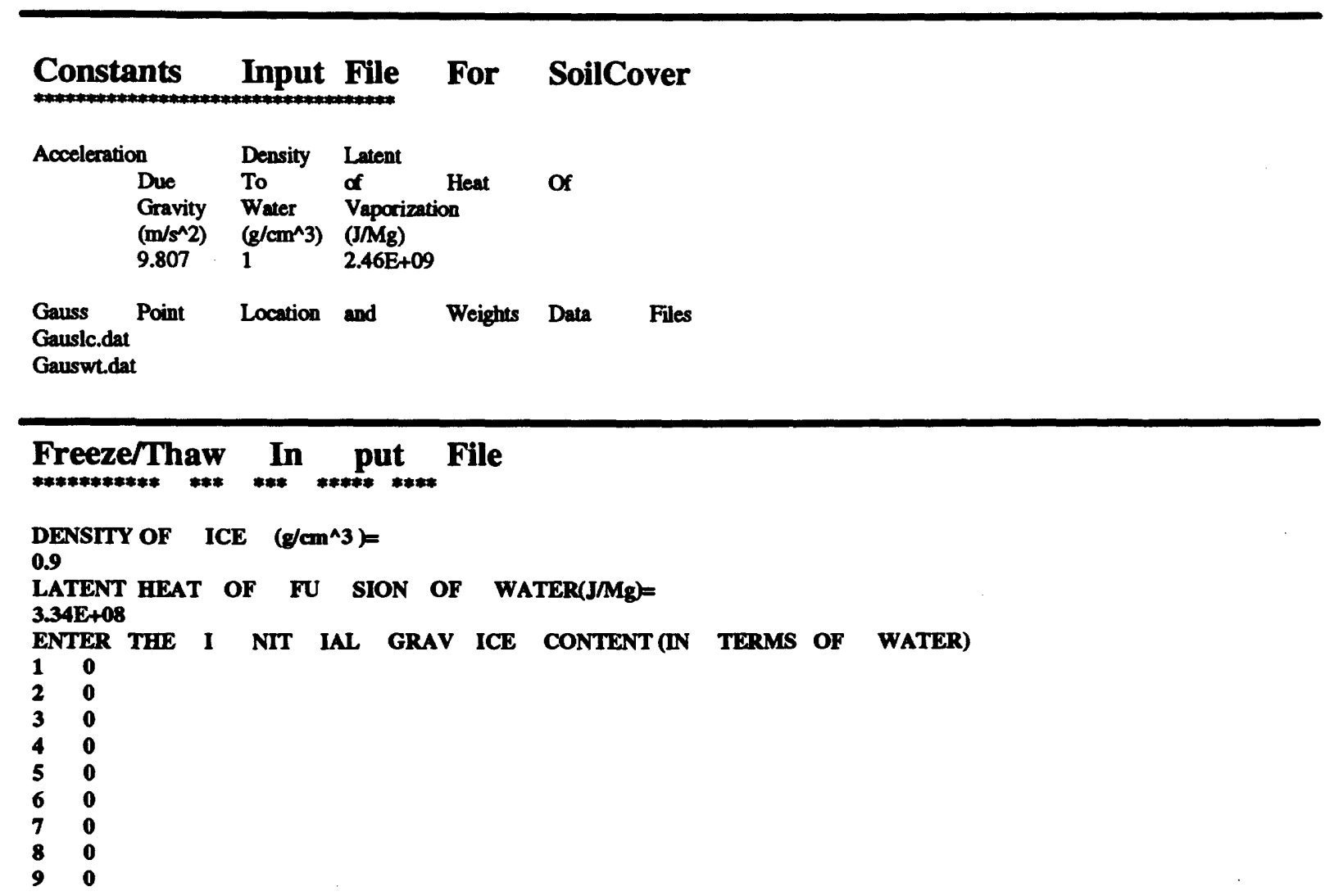




\begin{tabular}{ll}
10 & 0 \\
11 & 0 \\
12 & 0 \\
13 & 0 \\
14 & 0 \\
15 & 0 \\
16 & 0 \\
17 & 0 \\
18 & 0 \\
19 & 0 \\
20 & 0 \\
21 & 0 \\
22 & 0 \\
23 & 0 \\
24 & 0 \\
25 & 0 \\
26 & 0 \\
27 & 0 \\
28 & 0 \\
29 & 0 \\
30 & 0 \\
\hline 31 & 0 \\
32 & 0 \\
33 & 0
\end{tabular}

Soll Type \# 1

NUMBER OF D ATA PO INTS IN THE UNFROZEN W/C VS. TEMPERATURE CURVE FOR LAYER \#1

15

GRAV WAT Co nte nt NE G. TEMP

0.001840 .7696

0.0141211 .1918

$0.027663 \quad 74.42342$

0.054617 .97534

0.08113 .711712

$0.1 \quad 1.363568$

0.11920 .4728

0.140 .18018

0.14740 .118762

0.1590 .067568

0.16970 .045046

0.18880 .01926

0.2070 .009654

0.21450 .004504

0.220 .0009

number of data points in dsucditem vs volumetric water content function

18

Vol Wat dsuddtem

0.021110

0.0211110

0.0231110

0.0241110

0.0281110

0.0341110

0.0411110

0.0571110

0.0811110

0.1141110

0.1591110

0.2161110

0.2681110

$0.327 \quad 1110$

0.3791110

0.4341110

0.4641110

0.4921110

Soll Type \# 2 NUMBER OF D ATA PO INTS IN THE UNFROZEN W/C VS. TEMPERATURE CURVE FOR LAYER \#2 


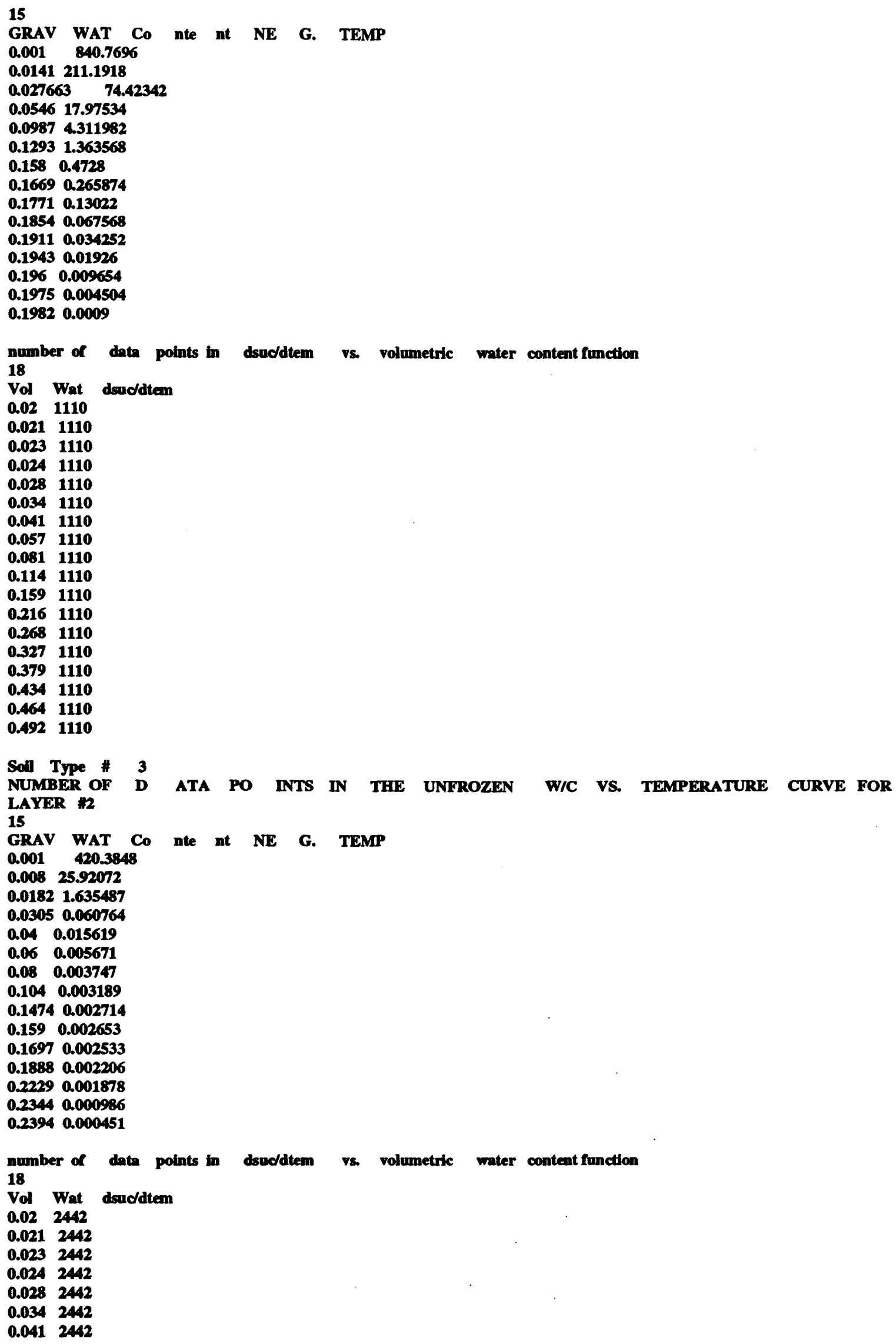




\section{Soil_Property_InputFile_for_SoilCover}

Soil_Type_\#1

$\begin{array}{ll}\text { Porosity } & \begin{array}{c}\text { Specfic } \\ \text { Gravity }\end{array} \\ 0.36 & 2.77\end{array}$

Moisture_Characteristic_Curve_For_Soil_Type_\#1

NumberOf Mv WaterContent

"DataPoints (1/kPa) Type(1=Gravimetric, $2=$ Volumetric)

$15 \quad 0.009061$

Suction WaterContent

(kPa) (dec)

10.22

$5 \quad 0.2145$

$\begin{array}{ll}10.71519 & 0.207\end{array}$

$21.37962 \quad 0.1888$

$\begin{array}{ll}50 & 0.1697\end{array}$

$75 \quad 0.159$

$131.8257 \quad 0.1474$

$200 \quad 0.14$

$524.8075 \quad 0.1192$

$1513.561 \quad 0.1$

$4120 \quad 0.0811$

$19952.62 \quad 0.0546$

826100.027663

$234422.9 \quad 0.0141$

$933254.3 \quad 0.001$

Hydraulic_Conductivity_Function_For_Soil_Type_\#1

Numberof a SatHydCond Imp. Factor

DataPoints (cm/s)

$10 \quad 2.00 \mathrm{E}-06 \quad 0$

Suction HydraulicConductivity

(kPa) $(\mathrm{cm} / \mathrm{s})$

$1 \quad 1.00 \mathrm{E}+00$

$10 \quad 8.67 \mathrm{E}-01$

$15.84893 \quad 2.75 \mathrm{E}-01$

$25.70396 \quad 7.45 \mathrm{E}-02$

$50.11872 \quad 8.71 \mathrm{E}-03$

$104.7129 \quad 1.12 \mathrm{E}-03$

$251.1886 \quad 1.58 \mathrm{E}-04$

$1584.893 \quad 2.00 \mathrm{E}-06$

$21877.62 \quad 1.29 \mathrm{E}-08$

467735.1 7.59E-11

Thermal_Conductivity_Function_For_Soil_Type_\#1

NumberOf WaterContent

"DataPoints Type(1=Gravimetric, $2=$ Volumetric) "

101

Water Thermal

Content Conductivity 

(dec) $(W / m \quad C)$
0.0050 .41
$\begin{array}{ll}0.02 & 0.8\end{array}$
$0.04 \quad 1.35$
$0.06 \quad 1.61$
$0.08 \quad 1.72$
$\begin{array}{ll}0.1 & 1.79\end{array}$
0.121 .86
0.141 .92
$\begin{array}{ll}0.16 & 1.99\end{array}$
0.168722 .02

Specific_Heat_Function_For_Soil_Type_\#1

NumberOf WaterContent

"DataPoints Type(1=Gravimetric,_2=Volumetric)

101

Water Specific

Content Heat

(dec.) (J/m^3-C)

0.0051469520

0.021601400

$0.04 \quad 1714440$

$0.06 \quad 1827480$

$0.08 \quad 1940520$

0.12053560

0.122147760

0.142260800

0.162355000

0.168722392680

Soil_Type_\#2

Porosity $\begin{gathered}\text { Specfic } \\ \text { Gravity }\end{gathered}$

$0.336 \quad 2.7$

Moisture_Characteristic_Curve_For_Soil_Type_\#2

Numberof Mv WaterContent

"DataPoints (1/kPa) Type(1=Gravimetric,2=Volumetric)

$15 \quad 0.009061$

Suction WaterContent

(kPa) (dec)

l 0.1982

50.1975

10.715190 .196

$21.37962 \quad 0.1943$

$38.01894 \quad 0.1911$

750.1854

144.5440 .1771

$\begin{array}{ll}295.1209 & 0.1669\end{array}$

$524.8075 \quad 0.158$

$1513.561 \quad 0.1293$

$\begin{array}{ll}4786.301 & 0.0987\end{array}$

$19952.62 \quad 0.0546$

$82610 \quad 0.027663$

$234422.9 \quad 0.0141$

933254.3 0.001

Hydraulic_Conductivity_Function_For_Soil_Type_\#2

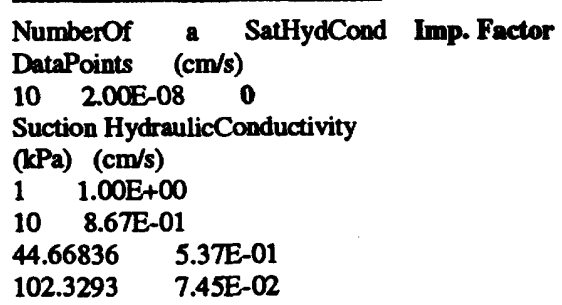




$\begin{array}{ll}281.8383 & 8.71 \mathrm{E}-03 \\ 794.3282 & 1.12 \mathrm{E}-03 \\ 2187.762 & 1.58 \mathrm{E}-04 \\ 16982.44 & 3.80 \mathrm{E}-06 \\ 102329.3 & 2.19 \mathrm{E}-07 \\ 436515.8 & 3.98 \mathrm{E}-08\end{array}$

Thermal_Conductivity_Function_For_Soil_Type_\#2

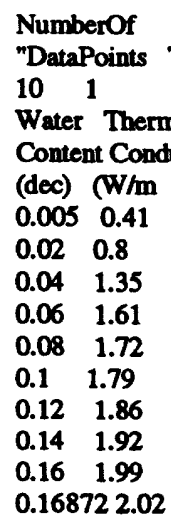

Specific_Heat_Function_For_Soil_Type_*2

NumberOf WaterContent

"DataPoints Type(1=Gravimetric, 2 =Volumetric)

101

Water Specific

Content Heat

(dec.) $\left(\mathrm{J} / \mathrm{m}^{\wedge} 3-\mathrm{C}\right)$

0.0051469520

0.021601400

$0.04 \quad 1714440$

$0.06 \quad 1827480$

$0.08 \quad 1940520$

0.12053560

0.122147760

0.142260800

0.162355000

0.168722392680

Soil_Type_\#3

Porosity $\begin{gathered}\text { Specfic } \\ \text { Gravity }\end{gathered}$

$0.4 \quad 2.65$

Moisture_Characteristic_Curve_For_Soil_Type_\#3 WaterContent

Number of $\mathbf{M v}$

"DataPoints g (1//kPa) Type(1=Gravimetric,2=Volumetric)

$15 \quad 0.009061$

Suction WaterContent

(kPa) (dec)

10.2394

$2.187762 \quad 0.2344$

$\begin{array}{ll}4.168694 & 0.2229\end{array}$

$4.897788 \quad 0.1888$

$\begin{array}{ll}5.495409 & 0.1697\end{array}$

$5.888437 \quad 0.159$

$\begin{array}{ll}6.309573 & 0.1474\end{array}$

$\begin{array}{ll}7.079458 & 0.104\end{array}$

$\begin{array}{ll}8.317638 & 0.08\end{array}$

$\begin{array}{ll}12.58925 & 0.06\end{array}$

$\begin{array}{ll}34.67369 & 0.04\end{array}$

$134.8963 \quad 0.0305$

$\begin{array}{ll}3630.781 & 0.0182\end{array}$ 
$57543.99 \quad 0.008$

$933254.3 \quad 0.001$

Hydraulic_Conductivity_Function_For_Soil_Type_\#3

NumberOf SatHydCond

DataPoints $(\mathrm{cm} / \mathrm{s})$

$10 \quad 3.00 \mathrm{E}-03 \quad 0$

Suction Hydraulic Conductivity

(kPa) $(\mathrm{cm} / \mathrm{s})$

$1 \quad 1.00 \mathrm{E}+00$

$3.235937 \quad 8.67 \mathrm{E}-01$

$5.058247 \quad 5.75 E-01$

$8.222426 \quad 2.09 \mathrm{E}-02$

$11.61449 \quad 2.51 \mathrm{E}-03$

14.79108 5.50E-04

19.6336 6.17E-05

28.31392 3.80E-06

38.90451 2.19E-07

$120.2264 \quad 1.17 \mathrm{E}-11$

Thermal_Conductivity_Function_For_Soil_Type_\#3

NumberOf WaterContent

"DataPoints h Type(1=Gravimetric,2=Volumetric) n

101

Water Thermal

Content Conductivity

(dec) $\left(\mathrm{W} / \mathrm{m}^{\wedge} 2\right)$

0.0050 .41

0.04810 .963

0.06621 .119

0.07861 .224

0.08831 .313

0.11 .396

$0.12 \quad 1.537$

0.141 .657

$\begin{array}{ll}0.16 & 1.761\end{array}$

$0.25 \quad 2.172$

Specific_Heat_Function_For_Soil_Type_\#3

NumberOf WaterContent

"DataPoints i Type(1=Gravimetric,_2=Volumetric)

101

Water Specific

Content Heat

(dec.) $\left(\mathrm{J} / \mathrm{m}^{\wedge} 3-\mathrm{C}\right)$

0.00661469520

0.021530000

0.041625000

$0.06 \quad 1719000$

$0.08 \quad 1806000$

0.11909000

0.121988000

0.142059000

0.18742262000

0.252475000

\section{Daily_Data_Input_File_For_SoilCover}

"Should_SoilCover_Use_Specified_Surface_Temperatures,_or_Calculate_it's_Own?_(0=Specified,1=Calculate)"

Total Temperature Rel.Humidity Latitude NumberOfDays

DaysOfData Lag Lag Past_January_1st 
$\begin{array}{lllll}181 & 0 & 0 & 56 & 320\end{array}$

Daily_Data

Day Max Min Net Max Min Wind TopBoundryCondition BotBoundryCondition Top Bottom Run AirTemp AirTemp Radiation RH RH Speed Type Value Duration Type Value Temperature Temp NextDayTemp (C) (C) $\left(\mathrm{Mg} / \mathrm{m}^{\wedge} 2\right.$-day) $(\mathrm{dec}) \quad(\mathrm{dec})(\mathrm{km} / \mathrm{hr})$

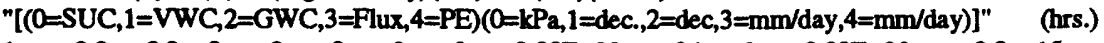

$\begin{array}{lllllllllllllll}1 & -0.2 & -0.2 & 0 & 0 & 0 & 0 & 3 & 0.00 \mathrm{E}+00 & 24 & 3 & 0.00 \mathrm{E}+00 & -0.2 & 15 & 1.5\end{array}$

$\begin{array}{lllllllllllllll}2 & 1.4 & 1.4 & 0 & 0 & 0 & 0 & 3 & 0.00 \mathrm{E}+00 & 24 & 3 & 0.00 \mathrm{E}+00 & 1.5 & 15 & -0.7\end{array}$

$\begin{array}{lllllllllllllll}3 & -0.9 & -0.9 & 0 & 0 & 0 & 0 & 3 & 0.00 \mathrm{E}+00 & 24 & 3 & 0.00 \mathrm{E}+00 & -0.7 & 15 & -3.7\end{array}$

$\begin{array}{lllllllllllllll}4 & -4 & -4 & 0 & 0 & 0 & 0 & 3 & 0.00 E+00 & 24 & 3 & 0.00 E+00 & -3.7 & 15 & -3\end{array}$

$\begin{array}{lllllllllllllll}5 & -3.4 & -3.4 & 0 & 0 & 0 & 0 & 3 & 0.00 E+00 & 24 & 3 & 0.00 E+\infty & -3 & 15 & 0.1\end{array}$

$\begin{array}{lllllllllllllll}6 & -0.4 & -0.4 & 0 & 0 & 0 & 0 & 3 & 0.00 E+00 & 24 & 3 & 0.00 \mathrm{E}+00 & 0.1 & 15 & -1.1\end{array}$

$\begin{array}{lllllllllllllll}7 & -1.7 & -1.7 & 0 & 0 & 0 & 0 & 3 & 0.00 \mathrm{E}+00 & 24 & 3 & 0.00 \mathrm{E}+00 & -1.1 & 15 & -0.9\end{array}$ 